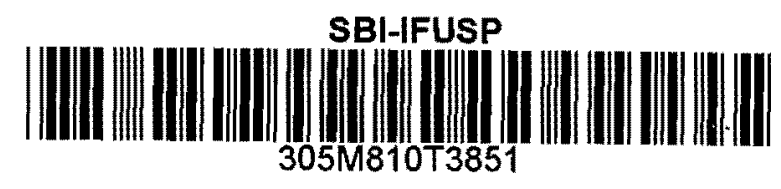

\author{
Universidade de São Paulo \\ Instituto de Física
}

\title{
Atalhos Gravitacionais em Cosmologia de Branas
}

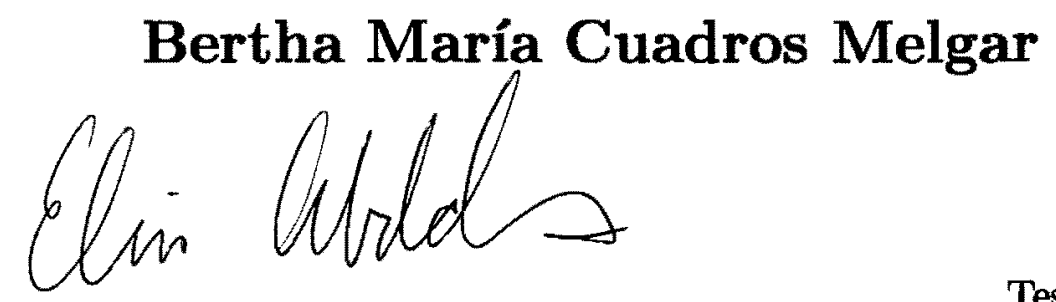

Tese de Doutorado apresentada ao Instituto de Física da Universidade de São Paulo

Comissão Examinadora:

Prof. Dr. Elcio Abdalla (IFUSP - São Paulo)

Prof. Dr. Fernando Tadeu Caldeira Brandt (IFUSP - São Paulo)

Prof. Dr. Josif Frenkel (IFUSP - São Paulo)

Prof. Dr. George Emanuel Avraam Matsas (IFT/UNESP - São Paulo)

Prof. Dr. Patricio Aníbal Letelier Sotomayor (UNICAMP - Campinas)

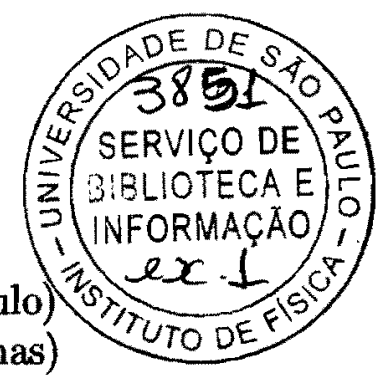

São Paulo 2003
Prof. A tmando Corbani Ferraz Presidente da Comissáo de Pós Gradueque 


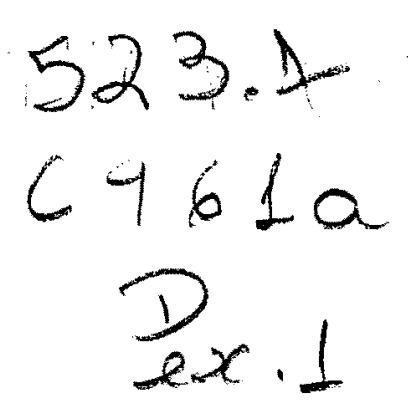

FICHA CATALOGRÁFICA

Preparada pelo Serviço de Biblioteca e Informação do Instituto de Física da Universidade de São Paulo

Cuadros Melgar, Bertha María

Atalhos Gravitacionais em Cosmologia de Branas. São Paulo, 2003

Tese (Doutoramento) Universidade de São Paulo Instituto de Física. Departamento de Física Matemática

Orientador: Prof. Dr. Elcio Abdalla

Área de Concentração: Física

Unitermos: 1. Dimensões extra;

2. Cosmologia; 3. Branas;

4. Gravitação. 


\section{Resumo}

Consideramos quatro modelos de branas, a saber, o modelo de Binétruy, Deffayet e Langlois com uma brana estática em um bulk dinâmico, o modelo de Chamblin e Reall para paredes de domínio dinâmicas, o modelo de brana estática em um espaçotempo AdS contendo uma singularidade, e a generalização do último caso, isto é, o modelo de brana dinâmica em um bulk AdS ou dS contendo uma singularidade nua ou protegida. Das equações de Einstein e das condições de Darmois-Israel, estudamos a dinâmica da 4-brana nos casos pertinentes. Usando o formalismo de Euler-Lagrange, derivamos uma equação para o caminho mais curto em 5 e 6 dimensões. Aplicando esta equação, investigamos a possibilidade de se ter atalhos, verificando sua existência nos três últimos modelos. Calculamos os atrasos relevantes entre os sinais do gráviton e do fóton e a razão dos horizontes subtendidos correspondentes. As conseqüências são discutidas. 


\section{Abstract}

We consider four brane world models, namely, the model of Binétruy, Deffayet and Langlois with a static brane in a dynamical bulk, the model of Chamblin and Reall for dynamic dilatonic domain walls, the static brane model in an AdS spacetime containing a shielded singularity, and the generalization of the latter case, i.e., the dynamic brane model in an AdS or dS bulk containing a naked or shielded singularity. From the Einstein equations and the Darmois-Israel conditions we study the 4-brane dynamics in the pertinent cases. Using the Euler-Lagrange formalism we derive an equation for the shortest path in 5 and 6 dimensions. Applying this equation we investigate the possibility of having shortcuts verifying its existence in the three last models. We calculate the relevant delay between graviton and photon signals and the ratio of the corresponding subtended horizons. Consequences are discussed. 
Aos meus adorados pais, Jorge e Teresa aos meus irmãos, Samuel, Milagros e Rosario ao meu sobrinho, Daniel aos meus melhores amigos, Melisa e Fidito. 


\section{Agradecimentos}

Gostaria de agradecer em primeiro lugar a meu orientador, Prof. Dr. Elcio Abdalla, que com muita paciência e dedicação guiou-me nestes inícios da minha carreira na cosmologia, e a quem considero não só um grande cientista, mas um grande amigo.

Agradeço também aos professores, funcionários e colegas do Departamento de Física Matemática, em especial, aos professores Fernando Brandt e Josif Frenkel, pela amizade e os muitos conselhos ao longo destes anos; a Adenauer Casali e Alejandro Correa-Borbonet, pelo muito que aprendi nas nossas discussões e pelo ânimo inestimável que sempre me deram; a João Luis Assirati, pela grande amizade e a paciência com meu computador; a Pablo, Leonardo, e Michele, pela grande compreensão e apoio.

Um agradecimento especial vai para meus colegas e amigos arequipeños no instituto, que iluminaram os tristes dias de saudade da nossa terra, Arequipa, e com quem passei inúmeros e inesquecíveis bons momentos nestas terras longínquas. Obrigada Mauricio e Liliana por serem os melhores camaradas que já conheci.

Não poderia esquecer de agradecer à grande torcida dos amigos de fora, espalhados pelo mundo todo: Melisa, Loreto, Leopardito, Otzé, Mike, Agaposhka, Marisa, Monika, Burin, Sergey, Vadim, Sashka, Wilfredo, Sussy, Moni, Rosita, Takeshi e em especial a meu grande amigo Bin, pelo trabalho em conjunto e pelos sábios conselhos repartidos.

Agradeço igualmente o grande apoio moral por parte da minha família, especialmente aos meus adorados pais, a quem devo tudo o que fui, sou e serei. A vocês dedico este humilde trabalho.

Um agradecimento também à FAPESP pelo apoio financeiro para a realização deste trabalho de pesquisa.

Finalmente, agradeço a Deus, a quem devo minha existência neste universo-brana. 


\section{Sumário}

1 Introdução 1

2 Atalhos Gravitacionais em 5 Dimensões 5

2.1 Modelos em 5 Dimensões . . . . . . . . . . . . . . 5

2.1.1 Modelos de Randall e Sundrum . . . . . . . . . . . . 5

2.1.2 Modelo de Binétruy, Deffayet e Langlois . . . . . . . . . . 7

2.2 O Caminho Mais Curto e o Deslocamento para o Vermelho . . . . . . 10

2.2.1 Equação Para o Caminho Mais Curto . . . . . . . . . . . . . 11

2.2.2 Deslocamento para o Vermelho para o Fóton e o Gráviton . . 14

2.3 Alguns Exemplos . . . . . . . . . . . . . . . . . 15

2.3.1 Solução de Vácuo RS . . . . . . . . . . . . . 15

2.3.2 Solução Cosmológica Linear BDL . . . . . . . . . . . . 15

3 Geodésicas no Cenário Cosmológico de Binétruy, Deffayet e Langlois 17

3.1 A Solução Cosmológica Linear . . . . . . . . . . . . . . . 17

3.2 A Solução Cosmológica Geral . . . . . . . . . . . . . . 18

3.3 Soluções da Equação Geodésica . . . . . . . . . . . . . . . . . 22

4 Atalhos Gravitacionais em 6 Dimensões 26

4.1 Modelo de Brana Estática . . . . . . . . . . . . . . . 26

4.2 Equação para os Atalhos . . . . . . . . . . . . . . . . 30

4.3 Modelos de Chamblin e Reall . . . . . . . . . . . . . . 32

4.3.1 Soluções Tipo-I . . . . . . . . . . . . . . . 35 
4.3 .2 Soluções Tipo-II . . . . . . . . . . . . . . . . . . . . 36

4.3 .3 Soluções Tipo-III . . . . . . . . . . . . . . . . . . . 37

4.4 Modelo de Brana Dinâmica . . . . . . . . . . . . . . . . . . 37

4.5 Equações de Friedmann $\ldots \ldots \ldots 41$

4.5.1 Dedução por Distribuições . . . . . . . . . . . . . . . . . 41

4.5.2 Dedução das Condições de Darmois-Israel . . . . . . . . 43

5 Atalhos no Modelo de Brana Estática 46

5.1 O Bulk AdS-Schwarzschild . . . . . . . . . . . . . . . . . 46

5.2 O Bulk AdS-Reissner-Nordström . . . . . . . . . . . . . . . . 51

5.2 .1 O Caso $k=0$ e $k=-1 \ldots \ldots \ldots \ldots \ldots \ldots$

$5.2 .2 \quad$ O Caso $k=1 \ldots \ldots \ldots \ldots \ldots \ldots \ldots \ldots$

5.3 Discussão dos Resultados . . . . . . . . . . . . . . . . . . . . . . 64

6 Atalhos em Paredes de Domínio e o Problema do Horizonte 66

6.1 O Cenário e a Equação de Atalhos . . . . . . . . . . . . . 66

6.2 Soluções da Equação Geodésica . . . . . . . . . . . . . . . . . 67

6.2 .1 Soluções Tipo-I . . . . . . . . . . . . . . . . . . 67

6.2 .2 Soluções Tipo-II . . . . . . . . . . . . . . . . . . . 71

6.2 .3 Soluções Tipo-III . . . . . . . . . . . . . . . . . 74

6.3 Tempo Próprio na Parede de Domínio e Atraso no Tempo $\ldots . . .79$

6.4 Discussão dos Resultados . . . . . . . . . . . . . . . . . . . . . 79

7 Dinâmica não Linear de uma Brana em um Modelo de 6 Dimensões 82

7.1 O Modelo, a Equação Geodésica e o Atraso do Tempo . . . . . . . . . 82

7.2 Flutuações na Posição da Brana . . . . . . . . . . . . . . . . 84

7.3 Dinâmica Não Linear da Brana . . . . . . . . . . . . . . . . 85

$7.3 .1 \quad M>0, Q^{2}=0, l^{2}>0 \ldots \ldots \ldots \ldots$

$7.3 .2 \quad M<0, Q^{2}=0, l^{2}>0 \ldots \ldots \ldots \ldots$

$7.3 .3 \quad M>0, Q^{2}=0, l^{2}<0 \ldots \ldots \ldots \ldots$ 
$7.3 .4 \quad M<0, Q^{2}=0, l^{2}<0 \ldots \ldots \ldots \ldots \ldots$

7.3.5 $M>0, Q^{2} \neq 0, l^{2}>0 \ldots \ldots \ldots \ldots 1$

7.3.6 $\quad M<0, Q^{2} \neq 0, l^{2}>0 \ldots \ldots \ldots \ldots$

$7.3 .7 \quad M>0, Q^{2} \neq 0, l^{2}<0 \ldots \ldots \ldots \ldots$

$7.3 .8 \quad M<0, Q^{2} \neq 0, l^{2}<0 \ldots \ldots \ldots \ldots$

7.4 Discussão dos Resultados . . . . . . . . . . . . . . . . . . . 96

$\begin{array}{lll}8 \text { Conclusões } & 98\end{array}$

$\begin{array}{ll}\text { A Geodésicas no Modelo BDL } & 101\end{array}$

B Atalhos no bulk AdS-Reissner-Nordström 106

C Solução das Equações no Modelo de Chamblin e Reall 109

D Atalhos no Modelo de Brana Dinâmica 114 


\section{Capítulo 1}

\section{Introdução}

A possibilidade de usar dimensões extras para explicar características relacionadas a teorias de campo unificadas foi utilizada há várias décadas por Kaluza e Klein $[1,2,3]$. Depois de um esquecimento de muitos anos, a idéia foi reestabelecida no contexto da supergravidade e da teoria de cordas. A idéia atual no que se conhece como Cosmologia de Branas, propõe que existam algumas dimensões espaciais extras em um cenário onde estas possam ser largas, sob a suposição de que a matéria ordinária esteja confinada no subespaço 3-dimensional, chamado de brana, imerso em um espaço dimensionalmente maior, chamado de bulk. A inspiração da cosmologia de branas tem sido o modelo sugerido por Hor̆ava e Witten $[4,5]$, algumas vezes chamado de Teoria $\mathrm{M}$, que descreve a teoria efetiva em baixas energias correspondente ao limite de acoplamento forte da teoria da corda heterótica $E_{8} \times E_{8}$. Este modelo descreve um espaço-tempo 11-dimensional com supergravidade em 11 dimensões, onde a dimensão 11 está compactificada por meio de uma simetria de orbifold $Z_{2}$. Dois pontos fixos da simetria orbifold definem duas bordas espaço-temporais em 10 dimensões, ou 9-branas, sobre as quais os grupos de gauge estão definidos. Desta configuração podemos distinguir três tipos de dimensões espaciais: a dimensão de orbifold, 3 dimensões largas que correspondem às direções espaciais ordinárias e, finalmente, 6 dimensões adicionais, que podem ser compactificadas da forma usual de Kaluza-Klein, isto é, em um raio do tamanho do comprimento de Planck $\left(\approx 10^{-33} \mathrm{~cm}\right)$, que evade qualquer verificação experimental atualmente ou em um futuro próximo. A dimensão de orbifold pode ser mais comprida que as 6 dimensões tipo Kaluza-Klein, resultando uma visão intermediária com um espaço-tempo 5-dimensional, duas 3-branas de borda (uma delas pode ser nosso universo), e uma dimensão extra larga. Assim, este modelo fornece a motivação para muitos dos modelos em cosmologia de branas.

Enquanto isso, outros problemas apareceram no esquema de teorias unificadas. Um deles é a grande hierarquia entre a escala eletrofraca $(\approx 100 \mathrm{GeV})$ e a escala de Planck $\left(\approx 10^{19} \mathrm{TeV}\right)$. Uma possível solução foi proposta por Arkani-Hamed, Dimopoulos e Dvali (ADD) [6]. Eles consideram um espaço-tempo $(4+n)$-dimensional 
com $n$ dimensões compactas com uma topologia de toro e um tamanho comum $R$. Da massa fundamental de Planck $(4+n)$-dimensional, $M_{*}$, que contém o acoplamento da gravitação à matéria desde um ponto de vista $(4+n)$-dimensional, é possível deduzir a massa de Planck efetiva em 4 dimensões, $M_{P}$,

$$
M_{P}^{2}=M_{*}^{2+n} R^{n} .
$$

Em escalas bem maiores que $R$, a gravitação $(4+n)$-dimensional se comporta efetivamente como nossa usual gravitação 4-dimensional, e as duas só podem ser distinguidas em escalas suficientemente pequenas, da ordem de $R$ ou menos. A observação simples de ADD é que uma massa fundamental $M_{*}$ da ordem da escala eletrofraca pode explicar a grande massa de Planck 4-dimensional $M_{P}$ que nós observamos, sempre que o volume nas dimensões extras seja muito grande. É claro que tal tamanho para as dimensões extras à la Kaluza-Klein é proibido pelas restrições experimentais, mas é permitido quando a matéria ordinária está confinada à 3-brana. As restrições no comportamento da gravitação são bem mais fracas já que a lei de Newton usual tem sido verificada experimentalmente só até uma fração de milímetro, o que deixa aberta a questão de se ter dimensões extras de tamanho submilimétrico.

A possibilidade de relaxar as restrições no tamanho das dimensões extras é muito atrativa. Tal é o caso do modelo de Randall e Sundrum $[7,8]$. Eles propuseram um modelo com um espaço-tempo Anti de Sitter 5-dimensional e mostraram que, para uma apropriada tensão na brana que representa nosso universo, é recuperada a gravitação 4-dimensional ainda com dimensões extras infinitas. As conseqüências cosmológicas deste modelo foram investigadas em vários trabalhos $[9,10,11,12,13$, $14,15,4,5,16,17,18,19,20,21,22,23]$, assim como os modelos com fatores de warp asimétricos [24] e sem simetria $Z_{2}$ [25]. Existe ainda um outro modelo [26] onde, contrariamente aos previamente citados, a gravitação torna-se 5-dimensional em largas escalas e pode ter aplicações interessantes na aceleração cosmológica atual $[27,28]$.

$\mathrm{O}$ assunto da alta dimensionalidade e suas conseqüências para o universo primordial tem também sido discutido na literatura recente $[16,17,18]$. Problemas relacionados à gravitação com derivadas altas [19] e à constante cosmológica [20] têm também sido estudados, assim como a correspondência AdS/CFT e a fórmula de Cardy [21].

Apesar dos aspectos atrativos do modelo, a causalidade pode ser violada, como foi notado primeiramente em [29, 30]. Temos duas escolhas para enfrentar esta situação: ou aceitamos que a causalidade deve estar definida pelas geodésicas no universo $(1+$ 4)-dimensional, em lugar do espaço-tempo $(1+3)$-dimensional, ou achamos algum mecanismo para evitar a violação na brana. No primeiro caso, a violação deve ser realmente pequena em experimentos de baixas energias, de outra forma já teria sido achada. A questão é se esta pode ser substancial para a cosmologia. Se a resposta for positiva, esta violação pode ajudar a resolver o bem conhecido problema do horizonte $[29,30]$. 
A existência de dimensões extras largas com um fator de warp naturalmente traz a questão de se a informação pode seguir um caminho mais curto fora da brana, sendo levada por grávitons [29, 30,31,32]. Motivados por esta idéia, propusemos um simples cálculo para estabelecer o caminho mais curto seguido pelo gráviton [33], que ao propagar-se em todas as dimensões no bulk, pode em princípio seguir uma trajetória que se desacople da brana (nosso universo), retornando mais tarde a ela em outro ponto, avançado no tempo com respeito ao fóton que, por construção, deve seguir uma trajetória na brana, um cenário que para um residente da brana (como nós) implica em atalhos $[34,24,29,30,31,33]$. O atraso do sinal eletromagnético frente às ondas gravitacionais, poderia então ser experimentalmente detectado com os detectores de ondas gravitacionais em curso [32, 35, 36].

Esta possibilidade implica que podemos ter alternativas ao cenário inflacionário para explicar o problema de homogeneidade em cosmologia. Recentemente, este cenário foi proposto como uma possibilidade realmente realizável [37, 38, 39, 40, 41]. Em [40] foi mostrado que em alguns cenários, os atalhos são difíceis de detectar hoje pelo atraso extremamente pequeno do fóton comparado com o gráviton vindos da mesma fonte. No entanto, nos casos onde antes da nucleosíntese, estes atrasos são suficientemente grandes para implicar em uma termalização do universo todo, uma solução possível ao problema de homogeneidade, alternativa [37, 38, 39, 41] à inflação [42], pode existir, baseada nestes atalhos. Ainda mais, se a inflação tomou parte na brana, a estrutura causal é definitivamente mudada pelos atalhos, levando possivelmente a períodos não usuais de evolução causal de escalas, o que poderia ser responsável pelas distintas predições na estrutura da radiação cósmica de fundo para os modelos inflacionários [40].

No caso de paredes de domínio, os atalhos são comuns e a possibilidade de resolver o problema de homogeneidade pode estar a caminho [40,43,44], mas certamente, são necessárias mais considerações em modelos mais realistas.

No próximo capítulo descreveremos os modelos em 5 dimensões relevantes ao nosso estudo e deduziremos a equação para o caminho mais curto, mostrando alguns exemplos simples de como usa-la. No capítulo 3, aplicaremos a equação geodésica deduzida anteriormente para o modelo de Binétruy, Deffayet e Langlois. Em seguida, no capítulo 4 detalharemos dois modelos em bulks AdS ou dS 6-dimensionais que propomos para o estudo de atalhos gravitacionais. Da mesma forma descreveremos o modelo de Chamblin e Reall para paredes de domínio imersas em $D$ dimensões. Neste mesmo capítulo, derivaremos a equação geodésica em 6 dimensões que dará origem aos atalhos gravitacionais mostrados ao longo da tese. As condições para a existência de atalhos no modelo de brana estática imersa em um bulk contendo uma singularidade protegida, serão abordadas no capítulo 5 . Logo, veremos no capítulo 6 como os atalhos aparecem no contexto de paredes de domínio em $D$ dimensões e calcularemos explicitamente o atraso no tempo para o fóton viajando na parede em relação ao gráviton viajando no bulk, que eventualmente pode ser medido por um observador na brana, como nós. No capítulo 7 iremos discutir a dinâmica não linear 
de uma brana imersa em um espaço-tempo AdS ou dS contendo uma singularidade nua ou protegida por ao menos um horizonte, cenário que representa a generalização do modelo mostrado no capítulo 5. Estudaremos também os atalhos que aparecem neste modelo e calcularemos o atraso no tempo de vôo do fóton em relação àquele do gráviton, e a razão entre os horizontes subtendidos por ambas as partículas. Finalmente, no último capítulo, discutiremos as conclusões e perspectivas do trabalho apresentado nesta tese de doutoramento. 


\section{Capítulo 2}

\section{Atalhos Gravitacionais em 5 Dimensões}

Neste capítulo vamos citar os principais modelos de branas em 5 dimensões relevantes para o presente trabalho, a saber, os modelos de Randall e Sundrum e os diversos cenários cosmológicos de Binétruy, Deffayet e Langlois. Derivaremos também a equação para o caminho mais curto no cenário multi-dimensional e definiremos o conceito de atalho neste contexto. Cabe mencionar que esta equaçâo (em 5 dimensões no presente capítulo e em 6 dimensões, como veremos no capítulo 4) é um dos principais resultados ao cujo redor giram todas as conseqüências físicas que serão apresentadas nesta tese de doutoramento. Finalmente, ilustraremos o uso desta equação com um par de exemplos simples, antes de passar a discutir os cenários mais complexos que irão aparecendo nos próximos capítulos.

\subsection{Modelos em 5 Dimensões}

\subsubsection{Modelos de Randall e Sundrum}

Se o espaço-tempo tem fundamentalmente mais de 4 dimensões, a escala de Planck 4-dimensional efetiva está determinada pela escala de Planck (4+n)-dimensional fundamental e a geometria das dimensões extras.

O fato de não vermos experimentalmente sinais das dimensões extras implica no fato das partículas e forças do modelo padrão, com exceção da gravidade, estarem confinadas a um subespaço 4-dimensional, imerso dentro de um outro espaço-tempo $(4+n)$-dimensional, chamado de 3-brana.

Motivados por fornecer uma aproximação alternativa ao problema de hierarquias, 
Randall e Sundrum propuseram uma solução das equações de Einstein em 5 dimensões em um cenário simples de duas 3-branas (RSI)(uma delas correspondendo ao nosso universo e a outra sendo uma brana "escondida") com termos cosmológicos apropriados [7]. Neste espaço, escalas de massa 4-dimensionais estão relacionadas a escalas mais fundamentais em 5 dimensões e a um fator de warp presente na métrica, que depende de um raio de compactificação que não precisa ser muito grande, desde que a fonte que gera a hierarquia entre as escalas de Planck e fraca, seja uma função exponencial dele.

A grande diferença entre a proposta de Randall e Sundrum com os trabalhos anteriores de Arkani-Hamed et. al. $[45,6]$ reside em que a hierarquia entre a escala fundamental de Planck 5-dimensional e a escala de compactificação é definida naturalmente no primeiro caso por um parâmetro da ordem de 10 , diferentemente da proposta dos últimos, onde a relação das escalas é $\left(M_{P l} / \mathrm{TeV}\right)^{2 / n}$. No primeiro caso só uma dimensão extra é necessária, frente às $n \geq 2$ dos demais modelos.

A ação clássica que descreve o cenário RS está dada por

$$
\begin{aligned}
S= & \int d^{4} x \int_{-\pi}^{\pi} d \phi \sqrt{-\tilde{g}}\left\{-\Lambda+2 M^{3} R\right\}+\int d^{4} x \sqrt{-g_{v i s}}\left\{\mathcal{L}_{v i s}-V_{v i s}\right\}+ \\
& +\int d^{4} x \sqrt{-g_{e s c}}\left\{\mathcal{L}_{e s c}-V_{e s c}\right\},
\end{aligned}
$$

onde $V_{v i s}$ e $V_{e s c}$ correspondem às energias de vácuo ou tensão das branas visível e escondida, respetivamente e $M$ define a escala fundamental em 5 dimensões.

O Ansatz proposto por Randall e Sundrum corresponde a uma métrica não fatorável em 5 dimensões, onde a métrica 4-dimensional está multiplicada por um fator de warp, que é uma função da coordenada adicional e muda rapidamente

$$
d s^{2}=e^{-2 \sigma(\phi)} \eta_{\mu \nu} d x^{\mu} d x^{\nu}+r_{c}^{2} d \phi^{2},
$$

onde $0 \leq \phi \leq \pi$ é a coordenada angular para a dimensão extra com raio de compactificação $r_{c}$. Requer-se que a coordenada seja periódica e que o espaço-tempo possua simetria $Z_{2}$. Assim, a quinta dimensão acha-se limitada entre duas 3-branas localizadas nos pontos fixos $\phi=0$ (uma brana escondida) e $\phi=\pi$ (nosso universo) do orbifold. Ambas as branas suportam teorias de campo em (3+1) dimensões.

Das equações de Einstein é possível determinar

$$
\sigma=r_{c}|\phi| \sqrt{\frac{-\Lambda}{24 M^{3}}},
$$

sendo $\Lambda<0$. A periodicidade da coordenada adicional implica que $V_{v i s}, V_{e s c}$ e $\Lambda$ estão relacionadas por uma única escala $k$ da ordem da escala de Planck:

$$
\begin{aligned}
V_{v i s} & =-V_{e s c}=-24 M^{3} k \\
\Lambda & =-24 M^{3} k^{2} .
\end{aligned}
$$


Finalmente, a métrica do bulk será

$$
d s^{2}=e^{-2 k r_{c}|\phi|} \eta_{\mu \nu} d x^{\mu} d x^{\nu}+r_{c}^{2} d \phi^{2}
$$

A hierarquia é gerada da seguinte forma. Consideramos flutuações gravitacionais sem massa ao redor da solução clássica (2.6) da forma $\bar{g}_{\mu \nu}=\eta_{\mu \nu}+\bar{h}_{\mu \nu}$, estas flutuações correspondem ao gráviton físico da teoria efetiva 4-dimensional (o modo zero da decomposição de $\tilde{g}_{\mu \nu}$ de Kaluza-Klein). Substituindo as flutuações na ação original (2.1), obtemos uma ação efetiva que inclui o termo seguinte

$$
S_{e f f} \supset \int d^{4} x \int_{-\pi}^{\pi} d \phi 2 M^{3} r_{c} e^{-2 k r_{c}|\phi|} \sqrt{-\bar{g}} \bar{R} .
$$

Integrando em $\phi$ para obter uma ação em 4 dimensões, podemos derivar a relação

$$
M_{P l}^{2}=M^{3} r_{c} \int_{-\pi}^{\pi} d \phi e^{-2 k r_{c}|\phi|}=\frac{M^{3}}{k}\left(1-e^{-2 k r_{c} \pi}\right) .
$$

Esta equação nos diz que $M_{P l}$ depende fracamente em $r_{c}$ no limite de $k r_{c}$ grande. Ainda que o exponencial tem um efeito pequeno na determinação da escala de Planck, notando que $g_{\mu \nu}^{v i s}=e^{-2 k r_{c} \pi} \bar{g}_{\mu \nu}$, é possível mostrar que qualquer parâmetro de massa $m_{0}$ na 3-brana visível corresponde a uma massa física $m \equiv e^{-k r_{c} \pi} m_{0}$ na teoria fundamental em mais dimensões, quando medida com a métrica $\bar{g}_{\mu \nu}$. Se $e^{k r_{c} \pi}$ é da ordem $10^{15}$, este mecanismo produz escalas de massa físicas de $\mathrm{TeV}$ a partir de parâmetros de massa fundamentais não longe da escala de Planck, $10^{19} \mathrm{GeV}$, requerendo somente $k r_{c} \approx 10$.

Posteriormente, foi notado que a tensão negativa da brana visível geraria uma gravidade efetiva repulsiva, o que não corresponde à realidade física. Assim, Randall e Sundrum propõem um segundo modelo (RSII) [8] invertendo os papéis das duas branas, tal que a brana visível possua uma tensão positiva. O ressaltante deste novo modelo é que a dimensão extra não é compacta (tomando o limite $r_{c} \rightarrow \infty$ na métrica do bulk e assim removendo a brana "escondida") e ainda temos uma escala de Planck finita e perfeitamente definida. Uma inevitável conseqüência do modelo é a existência de um estado ligado do gráviton ordinário 4-dimensional sem massa, com função de onda na dimensão extra centrada na 3-brana e cuja forma é determinada pela tensão da brana e a constante cosmológica do bulk. Assim, o fato de que o gráviton não massivo não se "espalhe" na dimensão extra, garante o sucesso da lei $1 / r^{2}$ de Newton.

\subsubsection{Modelo de Binétruy, Deffayet e Langlois}

Vamos considerar uma métrica em 5 dimensões descrevendo uma cosmologia de branas. Assim, escrevemos uma ação em 5 dimensões da forma [10]

$$
S^{(5)}=-\frac{1}{2 \kappa_{(5)}^{2}} \int d^{5} x \sqrt{-\tilde{g}} \tilde{R}+\int d^{5} x \sqrt{-\tilde{g}} \mathcal{L}_{m},
$$


onde o primeiro termo corresponde à ação de Einstein-Hilbert em 5 dimensões para uma métrica 5-dimensional $\tilde{g}_{A B}$, com $\tilde{R}$ sendo o escalar de curvatura 5-dimensional para esta métrica. O segundo termo corresponde à matéria. A convenção de índices aqui é a seguinte: letras latinas em maiúscula correspondem aos índices em 5 dimensões; letras gregas, aos índices em 4 dimensões; e letras latinas em minúscula, aos índices das dimensões espaciais ordinárias. A signatura da métrica é $(-++++)$. A constante $\kappa_{(5)}$ está relacionada à massa de Planck por $\kappa_{(5)}^{2}=M_{(5)}^{-3}$.

Já que nosso propósito é descrever uma cosmologia homogênea dentro de uma brana fina imersa em um espaço 5-dimensional, consideraremos as 3 dimensões espaciais ordinárias como sendo homogêneas e isotrópicas. A métrica mais conveniente, compatível com estas simetrias, pode ser sempre escrita em coordenadas normais gaussianas, tal que a métrica induzida na brana possa ser imediatamente obtida na forma de Friedmann-Lemaître-Robertson-Walker

$$
d s_{(5)}^{2}=-n^{2}(t, y) d t^{2}+a^{2}(t, y) \gamma_{i j} d x^{k} d x^{j}+b^{2}(t, y) d y^{2},
$$

onde $\gamma_{i j}$ representa uma métrica maximamente simétrica em 3 dimensões com $k=$ $-1,0,1$ parametrizando a curvatura espacial. A coordenada da quinta dimensão $y$ é compacta no intervalo $-1 / 2 \leq y \leq 1 / 2$, com os dois pontos extremos do intervalo identificados. Inspirada na compactificação da teoria de Hor̆ava-Witten [4, 5], é imposta a simetria $Z_{2}$ ou simetria de espelho $y \rightarrow-y$, e assim o intervalo relevante será $0 \leq y \leq 1 / 2$ com duas branas localizadas em $y=0$ e $y=1 / 2$ formando as bordas do espaço-tempo. Neste modelo, a hipersuperfície definica por $y=0$ corresponde ao nosso universo.

O tensor de energia-momento que aparece nas equações de Einstein $G_{A B}=\kappa_{(5)}^{2} \mathcal{T}_{A B}$ pode ser escrito como

$$
\mathcal{T}_{A B}=\hat{T}_{A B}+T_{A B},
$$

onde $\hat{T}_{A B}$ é o tensor de energia-momento da matéria do bulk (no cenário de Randall e Sundrum (RS) vem da constante cosmológica do bulk $\Lambda$, isto é, $\hat{T}_{A B}=-\Lambda \delta_{A B}$ ), e $T_{A B}$ corresponde ao conteúdo de matéria na brana localizada em $y=0$. Estamos interessados no caso onde o tensor de energia-momento da matéria na brana possa ser expresso como

$$
T_{B}^{A}=\frac{\delta(y)}{b} \operatorname{diag}(-\rho-\sigma, p-\sigma, p-\sigma, p-\sigma, 0) .
$$

Aqui $\sigma$ é a tensão da brana no cenário RS. A densidade de energia $\rho$ e a pressão $p$ vêm da matéria comum na brana e são independentes da posição.

As componentes não nulas do tensor de Einstein estão dadas por

$$
\tilde{G}_{00}=3\left\{\frac{\dot{a}}{a}\left(\frac{\dot{a}}{a}+\frac{\dot{b}}{b}\right)-\frac{n^{2}}{b^{2}}\left(\frac{a^{\prime \prime}}{a}+\frac{a^{\prime}}{a}\left(\frac{a^{\prime}}{a}-\frac{b^{\prime}}{b}\right)\right)+k \frac{n^{2}}{a^{2}}\right\},
$$




$$
\begin{aligned}
\tilde{G}_{i j}= & \frac{a^{2}}{b^{2}} \gamma_{i j}\left\{\frac{a^{\prime}}{a}\left(\frac{a^{\prime}}{a}+2 \frac{n^{\prime}}{n}\right)-\frac{b^{\prime}}{b}\left(\frac{n^{\prime}}{n}+2 \frac{a^{\prime}}{a}\right)+2 \frac{a^{\prime \prime}}{a}+\frac{n^{\prime \prime}}{n}\right\}+ \\
& +\frac{a^{2}}{n^{2}} \gamma_{i j}\left\{\frac{\dot{a}}{a}\left(-\frac{\dot{a}}{a}+2 \frac{\dot{n}}{n}\right)-2 \frac{\ddot{a}}{a}+\frac{\dot{b}}{b}\left(-2 \frac{\dot{a}}{a}+\frac{\dot{n}}{n}\right)-\frac{\ddot{b}}{b}\right\}-k \gamma_{i j}, \\
\tilde{G}_{05}= & 3\left(\frac{n^{\prime}}{n} \frac{\dot{a}}{a}+\frac{a^{\prime}}{a} \frac{\dot{b}}{b}-\frac{\dot{a}^{\prime}}{a}\right), \\
\tilde{G}_{55}= & 3\left\{\frac{a^{\prime}}{a}\left(\frac{a^{\prime}}{a}+\frac{n^{\prime}}{n}\right)-\frac{b^{2}}{n^{2}}\left(\frac{\dot{a}}{a}\left(\frac{\dot{a}}{a}-\frac{\dot{n}}{n}\right)+\frac{\ddot{a}}{a}\right)-k \frac{b^{2}}{a^{2}}\right\},
\end{aligned}
$$

onde a linha representa a derivada com respeito a $y$, e o ponto corresponde à derivada com respeito a $t$.

Procuramos agora uma solução às equações de Einstein na vizinhança de $y=0$. Para ter uma geometria bem definida, a métrica deve ser contínua através da brana. Porém, suas derivadas com respeito a $y$ podem ser descontínuas em $y=0$. Assim, existe uma função delta de Dirac na derivada segunda da métrica com respeito a $y$. Por tanto, os termos resultantes com uma função delta que apareçam no tensor de Einstein devem ser casados com as componentes distribucionais do tensor de energiamomento para satisfazer as equações de Einstein. Assim, usando as componentes $\tilde{G}_{00}$ e $\tilde{G}_{i j}$ obtemos as seguintes relações

$$
\begin{aligned}
\frac{\left[a^{\prime}\right]}{a_{0} b_{0}} & =-\frac{\kappa_{(5)}^{2}}{3}(\rho+\sigma), \\
\frac{\left[n^{\prime}\right]}{n_{0} b_{0}} & =\frac{\kappa_{(5)}^{2}}{3}(3 p+2 \rho-\sigma),
\end{aligned}
$$

conhecidas também como condições de Darmois-Israel [46] ou equações de junção (uma derivação alternativa será dada no capítulo 4), onde $\left[a^{\prime}\right]=a^{\prime}\left(0^{+}\right)-$ $a^{\prime}\left(0^{-}\right)$é o salto na derivada primeira através de $y=0$, e o subíndice ' 0 ' indica que os valores dos coeficientes da métrica devem ser tomados na brana.

Assumindo a simetria $\mathbf{Z}_{2}$ e $\sigma=0$, as equações de Einstein permitem uma solução cosmológica exata [10] (que corresponde a $\Lambda=0, \sigma=0, \gamma_{j k}=\delta_{j k}$ )

$$
\begin{aligned}
a & =a_{0}(t)(1+\lambda|y|), \\
n & =n_{0}(t)(1+\tilde{\mu}|y|), \\
b & =b_{0},
\end{aligned}
$$

onde impõe-se que $b_{0}$ seja uma constante no tempo (uma redefinição de $y$ pode fazer $b_{0}=1$ ) e $n_{0}(t)$ é uma função arbitrária (uma adequada redefinição de $t$ como o tempo cósmico ordinário, fixa-a em 1). $\lambda$ e $\tilde{\mu}$ são em geral funções do tempo e dependem diretamente da matéria na brana de acordo com (2.17):

$$
\lambda=-\frac{\kappa_{(5)}^{2}}{6} b_{0} \rho
$$




$$
\tilde{\mu}=\frac{\kappa_{(5)}^{2}}{2}\left(\omega+\frac{2}{3}\right) b_{0} \rho,
$$

onde $\kappa_{(5)}^{2}$ está relacionada à constante de Newton em 5 dimensões $G_{(5)}$ por $\kappa_{(5)}^{2}=$ $8 \pi G_{(5)}$. Especificando a equação de estado da matéria como na definição usual $p=\omega \rho$, usando novamente as equações de Einstein com o Ansatz (2.18), e a equação de conservação de energia na brana

$$
\dot{\rho}+3 \frac{\dot{a}_{0}}{a_{0}}(1+\omega) \rho=0
$$

que pode ser obtida das identidades de Bianchi $\nabla_{A} \tilde{G}_{B}^{A}=0$, é possível determinar as funções $a_{0}(t)$ e $\rho(t)$.

Para $\omega=-1$ temos o caso inflacionário,

$$
a_{0}(t) \propto e^{H t}, \quad H=\frac{\kappa_{(5)}^{2}}{6} \rho=\text { const. }
$$

enquanto que para $\omega \neq-1$, a solução conhecida para uma brana sem tensão é recuperada,

$$
a_{0} \propto t^{\tilde{q}}, \quad \kappa_{(5)}^{2} \rho=\frac{6 \tilde{q}}{t}, \quad \tilde{q}=\frac{1}{3(1+\omega)}
$$

É interessante notar que a solução exata do modelo RS pode ser também obtida [47], como veremos no próximo capítulo.

\subsection{O Caminho Mais Curto e o Deslocamento para o Vermelho}

Consideremos o problema seguinte [33]: suponha que existam dois observadores $A$ e $B$ na brana. $A$ pode mandar uma séries de fótons e grávitons a $B$ para estabelecer uma comunicação (veja Fig.2.1). De acordo com a cosmologia da brana, os fótons viajam na brana, enquanto os grávitons podem viajar no bulk. Consideremos então três questões:

1. Qual é o caminho mais curto para os grávitons e está ele na brana ou fora dela?

2. Quanto tempo antes os grávitons podem chegar a $B$ ?

3. Qual é a diferença do deslocamento ao vermelho para fótons e grávitons depois que eles chegaram a $B$ ? 


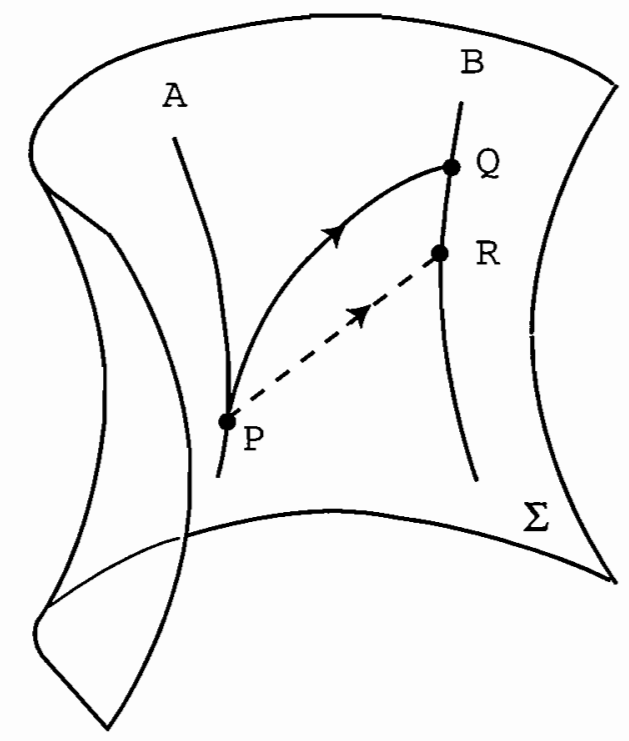

Figura 2.1: Duas possíveis trajetórias para a propagação de sinais sem massa. A curva sólida PQ é uma geodésica nula na brana $\Sigma$, e a linha quebrada PR é uma geodésica nula no bulk (modificado de [29]) .

\subsubsection{Equação Para o Caminho Mais Curto}

Para encontrar a equação para o caminho mais curto, vamos considerar a métrica genérica (2.10) para $b=1$. Consideremos dois pontos $r_{A}$ e $r_{B}$ na brana. Em geral, existe mais de uma geodésica conectando $r_{A}$ a $r_{B}$ no espaço-tempo de $1+4$ dimensões. No presente trabalho supõe-se que as trajetórias dos fótons devem estar na brana e aquelas dos grávitons podem estar fora. Consideremos o caminho mais curto tanto para fótons como para grávitons. Já que a métrica tridimensional é esfericamente simétrica e procuramos por trajetórias radiais, podemos omitir a parte angular e somente considerar o problema para

$$
d s_{3}^{2}=-n^{2}(t, y) d t^{2}+a^{2}(t, y) f^{2}(r) d r^{2}+d y^{2} .
$$

A trajetória do fóton está na brana $[n(t, 0)=1]$; portanto,

$$
-d t^{2}+a_{0}^{2}(t) f^{2}(r) d r^{2}=0,
$$

que pode ser imediatamente integrada como

$$
\int_{r_{A}}^{r} f\left(r^{\prime}\right) d r^{\prime}=\int_{t_{A}}^{t} \frac{d t^{\prime}}{a_{0}\left(t^{\prime}\right)}
$$

A trajetória do gráviton é definida em termos da equação geodésica

$$
-n^{2}(t, y) d t^{2}+a^{2}(t, y) f^{2}(r) d r^{2}+d y^{2}=0 \quad .
$$


Vamos supor que a trajetória esteja parametrizada por $y=y(t)$. Assim, a relação $r=r(t)$ é obtida por

$$
\int_{r_{A}}^{r} f\left(r^{\prime}\right) d r^{\prime}=\int_{t_{A}}^{t} \frac{\sqrt{n^{2}(t, y)-\dot{y}^{2}(t)}}{a(t, y)} d t
$$

Procuramos pela trajetória para a qual $t_{B}$ alcança o mínimo quando $r=r_{B}$. Com este propósito, consideramos o caso geral

$$
\int_{r_{A}}^{r_{B}} f\left(r^{\prime}\right) d r^{\prime}=\int_{t_{A}}^{t_{B}} \mathcal{L}[y(t), \dot{y}(t) ; t] d t
$$

Para uma trajetória adjacente $y=y(t)+\delta y(t)$, temos

$$
\int_{r_{A}}^{r_{B}} f\left(r^{\prime}\right) d r^{\prime}=\int_{t_{A}}^{t_{B}+\delta t_{B}} \mathcal{L}[y(t)+\delta y(t), \dot{y}(t)+\delta \dot{y}(t) ; t] d t
$$

portanto obtemos a condição usual

$$
-\delta t_{B} \mathcal{L}\left[y\left(t_{B}\right), \dot{y}\left(t_{B}\right) ; t_{B}\right]=\delta \int_{t_{A}}^{t_{B}} \mathcal{L}[y(t), \dot{y}(t) ; t] d t
$$

O problema é então transformado no problema de Euler-Lagrange

$$
\delta \int_{t_{A}}^{t_{B}} \mathcal{L}[y(t), \dot{y}(t) ; t] d t=0
$$

Neste caso,

$$
\mathcal{L}[y(t), \dot{y}(t) ; t]=\frac{\sqrt{n^{2}(t, y)-\dot{y}^{2}(t)}}{a(t, y)}
$$

e temos

$$
\begin{aligned}
& \frac{\partial \mathcal{L}}{\partial y}=-a^{-2} a^{\prime}\left(n^{2}-\dot{y}^{2}\right)^{1 / 2}+a^{-1}\left(n^{2}-\dot{y}^{2}\right)^{-1 / 2} n n^{\prime} \\
& \frac{\partial \mathcal{L}}{\partial \dot{y}}=-a^{-1}\left(n^{2}-\dot{y}^{2}\right)^{-1 / 2} \dot{y} .
\end{aligned}
$$

A equação de Euler-Lagrange é então

$$
\begin{gathered}
-\ddot{y}+\left(\frac{\dot{a}}{a}+\frac{\dot{n}}{n}\right) \dot{y}+\left(\frac{2 n^{\prime}}{n}-\frac{a^{\prime}}{a}\right) \dot{y}^{2}-\frac{\dot{a}}{a n^{2}} \dot{y}^{3} \\
+\left(\frac{a^{\prime}}{a} n^{2}-n n^{\prime}\right)=0 .
\end{gathered}
$$

Desta equação podemos ver que o caminho mais curto está na brana somente quando

$$
\frac{a^{\prime}}{a} n^{2}-n n^{\prime}=0
$$


isto é,

$$
\partial_{y}\left(\frac{a}{n}\right)=0
$$

Além disso, se existe uma solução, quando $y$ alcança o máximo (onde $\dot{y}=0$ e $\ddot{y}<0$ ), devemos ter

$$
-\ddot{y}+\left(\frac{a^{\prime}}{a} n^{2}-n n^{\prime}\right)=0
$$

Assim, $\frac{a^{\prime}}{a} n^{2}-n n^{\prime}$, isto é, $\partial_{y}\left(a n^{-1}\right)$ deve ser negativo neste ponto.

Neste contexto, nosso principal objetivo será procurar por uma solução de (2.35) começando na brana, viajando através do bulk, e voltando à brana depois de um tempo finito. Se esta solução representar o caminho mais curto, chamamo-la de atalho.

A equação é não linear e difícil de resolver. Não existe garantia da existência da solução procurada. Para obter uma solução com ambos extremos na brana, poderíamos fazer a expansão de Fourier

$$
\begin{aligned}
y(t)= & \sum_{l=1}^{+\infty} y_{l} \sin \frac{l \pi}{t_{g B}-t_{A}}\left(t-t_{A}\right), \\
a(t, y)= & A(y)+\sum_{l=1}^{+\infty}\left[a_{l}^{s}(y) \sin \frac{l \pi}{t_{g B}-t_{A}}\left(t-t_{A}\right)\right. \\
& \left.+a_{l}^{c}(y) \cos \frac{l \pi}{t_{g B}-t_{A}}\left(t-t_{A}\right)\right], \\
n(t, y)= & N(y)+\sum_{l=1}^{+\infty}\left[n_{l}^{s}(y) \sin \frac{l \pi}{t_{g B}-t_{A}}\left(t-t_{A}\right)\right. \\
& \left.+n_{l}^{c}(y) \cos \frac{l \pi}{t_{g B}-t_{A}}\left(t-t_{A}\right)\right],
\end{aligned}
$$

e então substituir de volta nas equações diferenciais para obter os coeficientes $y_{l}$. Aqui $t_{g B}$ é o tempo quando o gráviton chega a $r_{B}$, que é diferente do tempo $t_{\gamma B}$ quando o fóton chega em $r_{B}$. Ele devia ser determinado autoconsistentemente pela equação

$$
\int_{r_{A}}^{r_{B}} f\left(r^{\prime}\right) d r^{\prime}=\int_{t_{A}}^{t_{g B}} \frac{\sqrt{n^{2}(t, y)-\dot{y}^{2}(t)}}{a(t, y)} d t
$$

uma vez que a solução seja obtida. Porém, como veremos nos próximos capítulos, a equação geodésica (2.35) pode ser resolvida exatamente em alguns casos e em outros por cálculo numérico.

Se quisermos achar a trajetória para um gráviton tal que possa ir o mais longe dentro de um intervalo de tempo dado $\left[t_{A}, t_{B}\right]$, podemos usar também a equação de 
Euler-Lagrange. Assim, a diferença de comprimento entre as geodésicas para fótons e grávitons dentro de um intervalo de tempo dado pode ser calculada

$$
\begin{gathered}
\int_{r_{A}}^{r_{g}} f\left(r^{\prime}\right) d r^{\prime}=\int_{t_{A}}^{t_{B}} \frac{\sqrt{n^{2}(t, y)-\dot{y}^{2}(t)}}{a(t, y)} d t \\
\int_{r_{A}}^{r_{\gamma}} f\left(r^{\prime}\right) d r^{\prime}=\int_{t_{A}}^{t_{B}} \frac{d t^{\prime}}{a_{0}\left(t^{\prime}\right)} .
\end{gathered}
$$

\subsubsection{Deslocamento para o Vermelho para o Fóton e o Gráviton}

Em geral, se $A$ manda sinais sem massa em $x_{A}^{\mu}$ e $x_{A}^{\mu}+d x_{A}^{\mu}$, estes sinais alcançarão $B$ em $x_{B}^{\mu}$ e $x_{B}^{\mu}+d x_{B}^{\mu}$. A relação entre $x_{A}^{\mu}, x_{A}^{\mu}+d x_{A}^{\mu}$ e $x_{B}^{\mu}, x_{B}^{\mu}+d x_{B}^{\mu}$ pode ser obtida resolvendo a equação geodésica. Assim, o deslocamento para o vermelho do sinal é [48]

$$
\frac{\nu_{B}}{\nu_{A}}=\sqrt{\frac{g_{00}\left(x_{B}\right)}{g_{00}\left(x_{A}\right)}} \frac{g_{0 \mu}\left(x_{A}\right) d x_{A}^{\mu}}{g_{0 \nu}\left(x_{B}\right) d x_{B}^{\nu}}=\sqrt{\frac{g_{00}\left(x_{A}\right)}{g_{00}\left(x_{B}\right)}} \frac{d x_{A}^{0}}{d x_{B}^{0}}
$$

Para uma métrica estática como o caso da métrica de Schwarzschild, pode ser mostrado que $d x_{A}^{0}=d x_{B}^{0}$, portanto,

$$
\frac{\nu_{B}}{\nu_{A}}=\sqrt{\frac{g_{00}\left(x_{A}\right)}{g_{00}\left(x_{B}\right)}} .
$$

Para a métrica de Robertson-Walker, dependente do tempo, temos

$$
\frac{d x_{A}^{0}}{d x_{B}^{0}}=\frac{R\left(x_{A}^{0}\right)}{R\left(x_{B}^{0}\right)},
$$

em cujo caso o deslocamento para o vermelho é dado por

$$
\frac{\nu_{B}}{\nu_{A}}=\frac{R\left(x_{A}^{0}\right)}{R\left(x_{B}^{0}\right)} .
$$

Assim, no limite óptico-geométrico, os deslocamentos para o vermelho em ambos casos podem ser sistematicamente discutidos.

Aqui consideramos que um outro gráviton começa viajando desde $r_{A}$ em um tempo mais tarde $t_{A}+\delta t_{A}$. Seu caminho mais curto é em geral diferente do anterior. Denotemos este por $y_{*}=y_{*}(t)$. Então o tempo quando ele chega em $r_{B}$ será um tempo posterior $t_{g B}+\delta t_{g B}$

$$
\int_{r_{A}}^{r_{B}} f\left(r^{\prime}\right) d r^{\prime}=\int_{t_{A}+\delta t_{A}}^{t_{g B}+\delta t_{g_{B}}} \frac{\sqrt{n^{2}\left(t, y_{*}\right)-{\dot{y_{*}}}^{2}(t)}}{a\left(t, y_{*}\right)} d t .
$$


Portanto, temos a igualdade

$$
\int_{t_{A}}^{t_{g B}} \frac{\sqrt{n^{2}(t, y)-\dot{y}^{2}(t)}}{a(t, y)} d t=\int_{t_{A}+\delta t_{A}}^{t_{g B}+\delta t_{g B}} \frac{\sqrt{n^{2}\left(t, y_{*}\right)-{\dot{y_{*}}}^{2}(t)}}{a\left(t, y_{*}\right)} d t
$$

Para um infinitesimal $d t_{A}$ e $d t_{B}$, temos

$$
\left.d t_{B}\left(\frac{\sqrt{n^{2}(t, y)-\dot{y}^{2}(t)}}{a(t, y)}\right)\right|_{B}=\left.d t_{A}\left(\frac{\sqrt{n^{2}(t, y)-\dot{y}^{2}(t)}}{a(t, y)}\right)\right|_{A}
$$

Assim, o deslocamento para o vermelho do gráviton é dado por

$$
\frac{\nu_{g B}}{\nu_{g A}}=\frac{a_{0}\left(t_{A}\right)}{a_{0}\left(t_{B}\right)} \sqrt{\frac{1-\dot{y}^{2}\left(t_{B}\right)}{1-\dot{y}^{2}\left(t_{A}\right)}}
$$

enquanto que para o fóton temos

$$
\frac{\nu_{g B}}{\nu_{g A}}=\frac{a_{0}\left(t_{A}\right)}{a_{0}\left(t_{B}\right)}
$$

\subsection{Alguns Exemplos}

\subsubsection{Solução de Vácuo RS}

Neste caso, da Eq. (2.6)

$$
n(y, t)=a(y, t)=e^{-k|y|}
$$

A Eq. (2.35) torna-se

$$
\ddot{y}+k \dot{y}^{2}=0 .
$$

Que tem duas possíveis soluções: uma é $y=y_{A}=0$, e a outra é $y=y_{0}+k^{-1} \ln \left(t-t_{0}\right)$. A segunda solução não cumpre com a nossa imposição já que não acaba na brana. Assim, o caminho mais curto deve estar na brana. Isto concorda com a conclusão em $[29]$.

\subsubsection{Solução Cosmológica Linear BDL}

Primeiramente consideramos em (2.18) o caso $\omega=-\frac{2}{3}$, tal que da Eq. (2.20), $\tilde{\mu}=0$, $a(t, y)=t-y, \lambda=-\frac{1}{t}$. A equação é

$$
-(t-y) \ddot{y}+\dot{y}+\dot{y}^{2}-\dot{y}^{3}-1=0
$$

Seja $t-y=u$, então

$$
u \ddot{u}+\dot{u}^{3}-2 \dot{u}^{2}=0
$$


ou

$$
\frac{1}{2 \dot{u}^{2}-\dot{u}^{3}} \frac{d}{d t} \dot{u}^{2}=\frac{2 \dot{u}}{u}
$$

Portanto,

$$
\begin{aligned}
\int \frac{d \dot{u}}{2 \dot{u}-\dot{u}^{2}} & =\int \frac{d u}{u} \\
\frac{\dot{u}}{2-\dot{u}} & =c u^{2} .
\end{aligned}
$$

Podemos obter a solução ( $t_{0}$ e $c$ são duas constantes de integração)

$$
y=t_{0} \pm \sqrt{\left(t-t_{0}\right)^{2}+\frac{1}{c}}
$$

É obvio que esta trajetória também não pode acabar na brana.

Vamos considerar agora o caso $\omega=-1, \lambda=\tilde{\mu}=$ const., $a_{0}(t)=e^{H t}$. Mas já que $\partial_{y}(a / n)=0$, o caminho mais curto continua estando na brana. 


\section{Capítulo 3}

\section{Geodésicas no Cenário Cosmológico de Binétruy, Deffayet e Langlois}

Os modelos considerados por Binétruy et. al. [10,47] descrevem nosso universo como uma brana 3-dimensional infinitesimalmente fina com curvatura constante imersa em um espaço-tempo 5-dimensional com constante cosmológica. Para uma equação de estado $p=\omega \rho$, eles acharam soluções explícitas que usaremos no estudo da questão da existência de atalhos, isto é, geodésicas correspondentes a grávitons que saem da brana para o bulk e voltam a ela antes que um fóton, viajando sobre a brana, tenha chegado no mesmo ponto. Vamos considerar primeiramente uma situação geral do modelo BDL estudado no capítulo anterior. Veremos que a equação geodésica resultante é difícil de resolver pela não linearidade que apresenta. Assim, passaremos a descrever uma outra solução cosmológica achada pelos mesmos autores em um trabalho posterior e que, em certo limite, reproduz a solução de Randall e Sundrum. Para este modelo, estudaremos o comportamento das geodésicas e tentaremos construir atalhos gravitacionais.

\subsection{A Solução Cosmológica Linear}

Como vimos no capítulo anterior, é possível obter algumas conclusões sobre onde se encontra o caminho mais curto em um cenário com dimensões extras para certos valores dos parâmetros. No caso do cenário cosmológico BDL vimos que nos casos $\omega=-2 / 3,-1$, o caminho mais curto encontra-se na brana. Consideremos agora $o$ caso geral $\omega \neq-1$ em $(2.18)$

$$
a_{0}(t)=t^{\bar{q}}, \quad \lambda=-\frac{\tilde{q}}{t}, \quad \tilde{\mu}=w \frac{\tilde{q}}{t}, \quad w=2+3 \omega
$$




$$
a(t, y)=t^{\tilde{q}}-\tilde{q} t^{\tilde{q}-1} y \quad, \quad n(t, y)=1+\frac{\tilde{q} \omega}{t} y
$$

Se fizermos $y=t f(t)$ em (2.35), tentando simplificar o problema, obtemos uma equação diferencial não linear

$$
\begin{aligned}
& -\left[1+(2 \tilde{q} \omega-\tilde{q}) f+\left(\tilde{q}^{2} \omega^{2}-2 \tilde{q}^{2} \omega^{2}\right) f^{2}-\tilde{q}^{3} \omega^{2} f^{3}\right]\left(t^{2} \ddot{f}+2 t \dot{f}\right) \\
& +\left[\tilde{q}+\left(2 \omega \tilde{q}^{2}-\tilde{q}^{2}+\tilde{q}-\tilde{q} \omega\right) f+\left(\tilde{q}^{2} \omega-\tilde{q}^{2} \omega^{2}-2 \omega \tilde{q}^{3}+2 \omega \tilde{q}^{2}\right) f^{2}\right. \\
& \left.\left.+\left(2 \tilde{q}^{3} \omega^{2}-\tilde{q}^{4} \omega^{2}\right) f^{3}\right](t \dot{f}+f)+[2 \tilde{q} \omega-\tilde{q})+\tilde{q}^{3} \omega^{2} f^{2}\right](t \dot{f}+f)^{2} \\
& -[\tilde{q}-\tilde{q}(\tilde{q}-1) f](t \dot{f}+f)^{3}+\left[(-\tilde{q}-\tilde{q} \omega)+\left(\tilde{q}^{2}-4 \tilde{q}^{2}-3 \tilde{q}^{2} \omega^{2}\right) f\right. \\
& +\left(3 \tilde{q}^{3} \omega-6 \tilde{q}^{3} \omega^{2}-3 \tilde{q}^{3} \omega^{3}\right) f^{2}+\left(-2 \tilde{q}^{4} \omega^{3}+3 \tilde{q}^{4}-\tilde{q}^{4} \omega^{4}\right) f^{3} \\
& \left.+\left(\tilde{q}^{5} \omega^{3}-\tilde{q}^{5} \omega^{4}\right) f^{4}\right]=0
\end{aligned}
$$

A análise desta equação diferencial pode ser feita usando algumas técnicas de sistemas dinâmicos, porém essa discussão está além do objetivo do presente trabalho. O que será feito é discutir alguns casos simples onde uma análise numérica possa ser feita.

\subsection{A Solução Cosmológica Geral}

O fato de assumir $\mathcal{T}_{05}=0$, que fisicamente diz que não existe fluxo de matéria ao longo da quinta dimensão, implica em $\tilde{G}_{05}=0$. Adicionalmente se considerarmos uma constante cosmológica $\rho_{B}=\Lambda$ como a única fonte no bulk, é possível obter uma integral primeira das equações de Einstein que, com a ajuda das equações de junção (2.17), produz a equação de Friedmann generalizada em 5 dimensões [47]:

$$
H^{2} \equiv \frac{\dot{a}_{0}^{2}}{a_{0}^{2}}=\frac{\kappa_{(5)}^{2}}{6} \rho_{B}+\frac{\kappa_{(5)}^{4}}{36} \rho_{b}^{2}+\frac{\mathcal{C}}{a_{0}^{4}}-\frac{k}{a_{0}^{2}}
$$

onde $\mathcal{C}$ é uma constante de integração. Note que os parâmetros $\rho_{b}, p_{b}, \rho_{\Lambda}$, e $q[47]$ nesta seção estão relacionados com os correspondentes no capítulo anterior pelas equações seguintes: $\rho_{b}=\rho+\sigma, p_{b}=p-\sigma, \rho_{\Lambda}=\sigma, q=\tilde{q}^{-1}$.

A característica mais marcante de (3.5) é que a densidade de energia na brana entra quadraticamente no lado direito da equação, em contraste com a equação de Friedmann padrão em 4 dimensões, $H^{2}=(8 \pi G / 3) \rho$, onde a densidade de energia entra linearmente.

Escolhendo uma quinta dimensão estática $(\dot{b}=0)$, a solução para o fator de escala, pode ser escrita como

$$
a(t, y)=\left\{\frac{1}{2}\left(1+\frac{\kappa_{(5)}^{2} \rho_{b}^{2}}{6 \rho_{B}}\right) a_{0}^{2}+\frac{3 \mathcal{C}}{\kappa_{(5)}^{2} \rho_{B} a_{0}^{2}}+\left[\frac{1}{2}\left(1-\frac{\kappa_{(5)}^{2} \rho_{b}^{2}}{6 \rho_{B}}\right) a_{0}^{2}-\right.\right.
$$




$$
\left.\left.-\frac{3 \mathcal{C}}{\kappa_{(5)}^{2} \rho_{B} a_{0}^{2}}\right] \cosh (\mu y)-\frac{\kappa_{(5)} \rho_{b}}{\sqrt{-6 \rho_{B}}} a_{0}^{2} \sinh (\mu|y|)\right\}^{1 / 2},
$$

onde $\mu$ está dado por

$$
\mu=\sqrt{-\frac{2 \kappa_{(5)}^{2}}{3} \rho_{B}} .
$$

A função $n(t, y)$ pode ser calculada da maneira seguinte:

$$
n(t, y)=\frac{\dot{a}(t, y)}{\dot{a}_{0}(t)} .
$$

Brevemente, notemos que a solução para o caso especial $\rho_{B}=0$ e $\mathcal{C}=0$, corresponde à solução cosmológica linear achada no capítulo 2 .

Voltemos agora à discussão da equação de Friedmann (3.5). O termo quadrático na densidade de energia conduz a uma cosmologia não convencional [10]. Porém, seguindo $[12,11]$, é possível implementar a idéia de Randall e Sundrum no contexto cosmológico para recuperar a cosmologia padrão. Escrevemos a densidade total de energia na brana $\rho_{b}$ como no capítulo 2 :

$$
\rho_{b}=\rho_{\Lambda}+\rho,
$$

com $\rho_{\Lambda}$ a tensão intrínseca da brana e $\rho$ a densidade de energia da matéria cosmológica ordinária. Substituindo em (3.5) obtemos

$$
H^{2} \equiv \frac{\dot{a}_{0}^{2}}{a_{0}^{2}}=\frac{\kappa_{(5)}^{2}}{6} \rho_{B}+\frac{\kappa_{(5)}^{4}}{36} \rho_{\Lambda}^{2}+\frac{\kappa_{(5)}^{4}}{18} \rho_{\Lambda} \rho+\frac{\kappa_{(5)}^{4}}{36} \rho^{2}+\frac{\mathcal{C}}{a_{0}^{4}}-\frac{k}{a_{0}^{2}}
$$

Se seguimos os trabalhos de Randall e Sundrum $[7,8]$, escolhendo $\rho_{\Lambda}$ tal que

$$
\frac{\kappa_{(5)}^{2}}{6} \rho_{B}+\frac{\kappa_{(5)}^{4}}{36} \rho_{\Lambda}^{2}=0
$$

vemos que a cosmologia padrão é recuperada com a identificação

$$
\kappa_{(4)}^{2} \equiv 8 \pi G=\frac{\kappa_{(5)}^{4}}{6} \rho_{\Lambda} .
$$

Assim, o terceiro termo no lado direito de Eq. (3.10) chega a ser dominante frente ao quarto termo se $\rho \ll \rho_{\Lambda}$, e corresponde exatamente ao termo linear na equação de Friedmann usual. $O$ quarto termo, quadrático na energia, fornece assim uma correção de altas energias à equação de Friedmann, e se torna significativo quando o valor da densidade de energia se aproxima ao valor da tensão $\rho_{\Lambda}$ e domina ainda em altas densidades de energia. No regime de altas energia, $\rho \gg \rho_{\Lambda}$, recuperamos 
o comportamento não convencional achado em [10], que não é surpresa já que a constante cosmológica do bulk é então desprezível.

Finalmente, o quinto termo em (3.10) comporta-se como radiação. A constante $\mathcal{C}$ é um análogo à massa de Schwarzschild, como será visto posteriormente, e está relacionada ao tensor de Weyl no bulk, que é nulo quando $\mathcal{C}=0$. No contexto cosmológico, este termo deve ser suficientemente pequeno no tempo da nucleosíntese para satisfazer às restrições do número de graus de liberdade extra leves. Na era de domínio da matéria, este termo se desloca ao vermelho rapidamente e seria, em princípio, desprezível hoje.

Para obter uma solução analítica de (3.10), assumimos que a matéria ordinária está descrita pela equação de estado usual $p=\omega \rho$. Usando a equação de conservação de energia na brana (2.21), podemos escrever

$$
\rho=\rho_{\star}\left(a_{0} / a_{\star}\right)^{-q} \quad, \quad q=3(1+\omega)
$$

Substituindo de volta na equação de Friedmann, esta pode ser integrada no caso $\mathcal{C}=0, k=0$. Definindo

$$
\lambda=\sqrt{\frac{\rho_{B}}{6 \kappa_{(5)}^{2}}+\frac{\rho_{\Lambda}^{2}}{36}}
$$

como sendo positivo, achamos que

$$
a_{0}(t)=a_{\star} \rho_{\star}^{1 / q}\left\{\frac{\rho_{\Lambda}}{36 \lambda^{2}}\left[\cosh \left(\kappa_{(5)}^{2} \lambda t q\right)-1\right]+\frac{1}{6 \lambda} \sinh \left(\kappa_{(5)}^{2} \lambda t q\right)\right\}^{1 / q} .
$$

Neste caso, o universo passa por três fases sucessivas: uma fase não convencional dominada por $\rho^{2}$, uma fase convencional dominada por $\rho$ e, finalmente, uma fase exponencial, onde $\lambda$ faz o papel de uma constante cosmológica efetiva para o nosso universo. Portanto, a cosmologia de branas é capaz de produzir uma fase acelerada em tempos tardios, como parece ser necessário das últimas observações cosmológicas $[49,50]$, mas ao preço de um ajuste fino entre a constante cosmológica do bulk e a tensão da brana, como vimos anteriormente, que pode ser identificado como a versão em cosmologia de branas do bem conhecido problema da constante cosmológica.

Para $\lambda=0$, que é o caso do modelo RS,

$$
a_{0}(t)=a_{\star}\left(\kappa_{(5)}^{2} \rho_{\star}\right)^{1 / q}\left(\frac{q^{2}}{72} \kappa_{(5)}^{2} \rho_{\Lambda} t^{2}+\frac{q}{6} t\right)^{1 / q}
$$

onde $a_{\star}, \rho_{\star}$ são constantes (a origem do tempo é escolhida tal que $a_{0}(0)=0$ ). É claro deste caso que o universo primitivo é caracterizado por uma evolução não convencional $a(t) \sim t^{1 / q}$, enquanto que em tempos maiores, o universo alcança a fase $a(t) \sim t^{2 / q}$, descrita pela cosmologia padrão. 


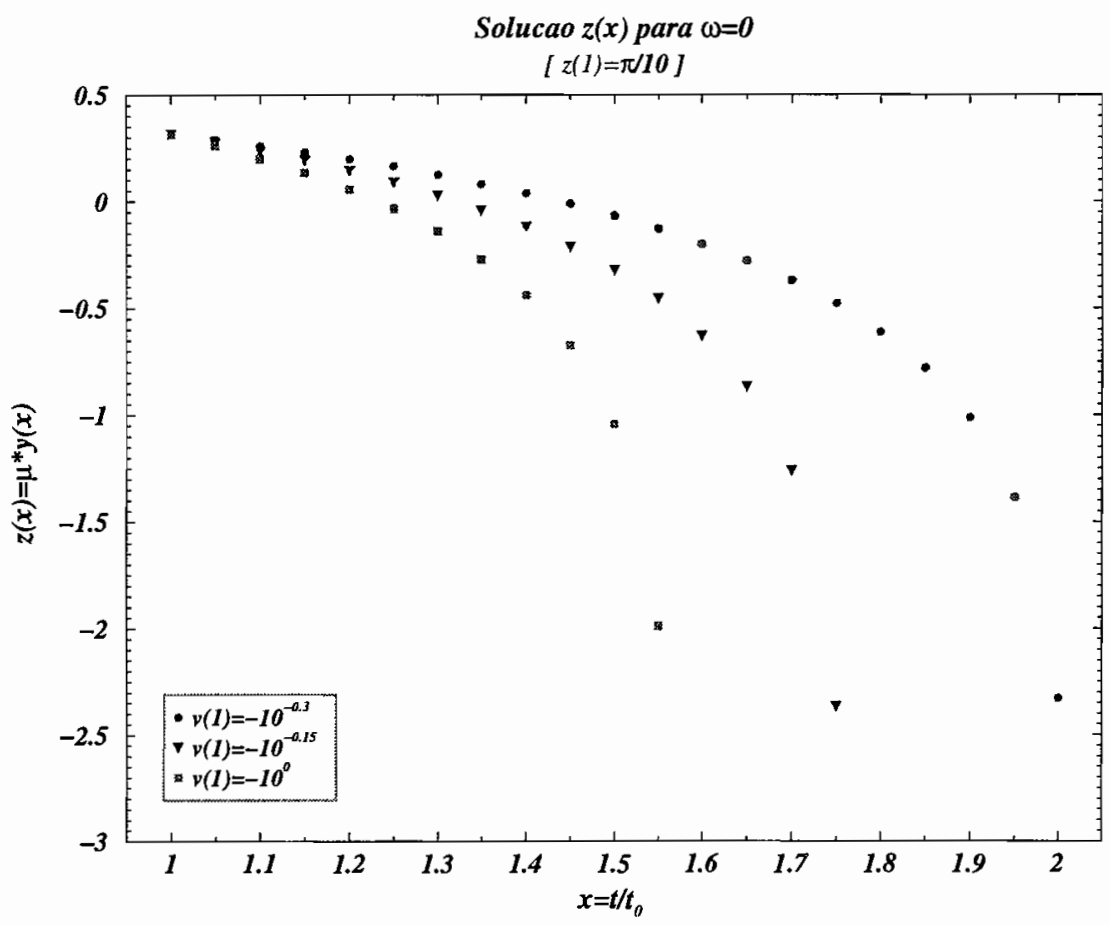

Figura 3.1: Gráfico para $y(1) \sim 0.3 \ell_{P}$.

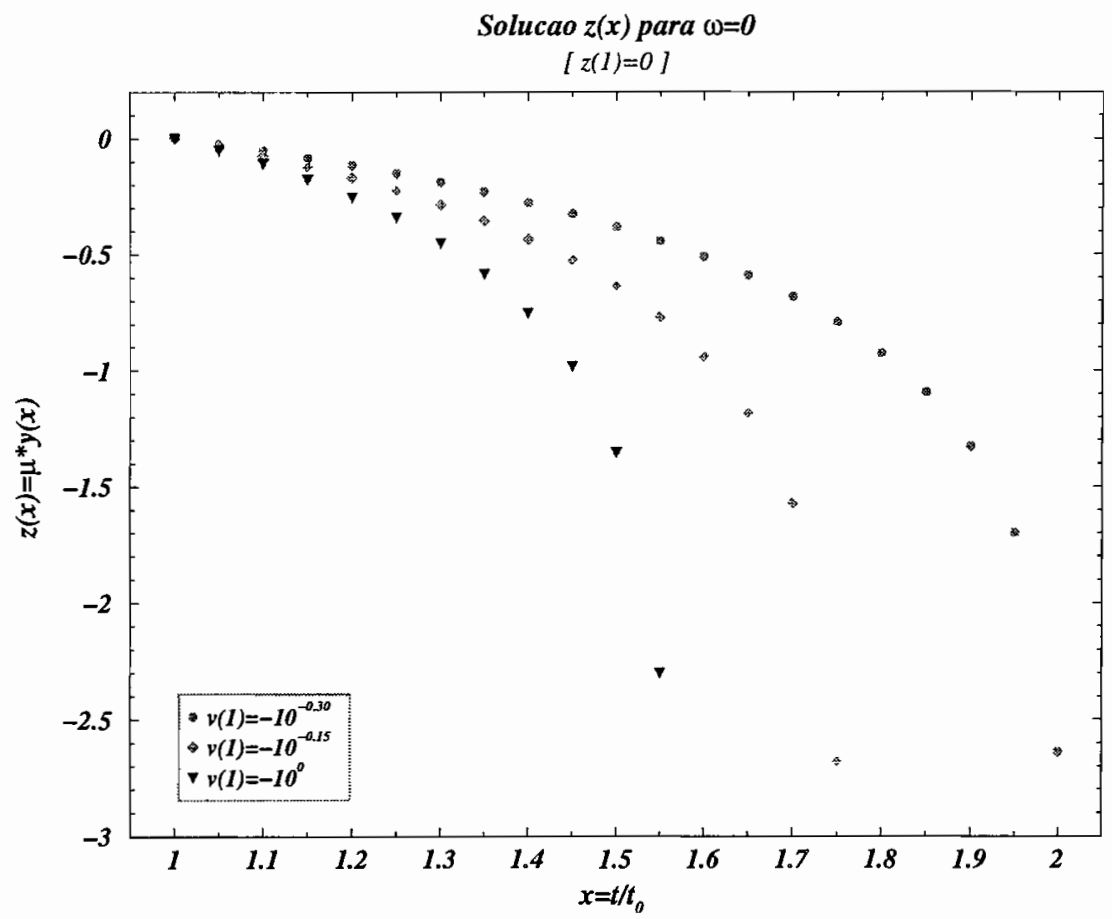

Figura 3.2: O mesmo gráfico que antes com y começando na brana. 


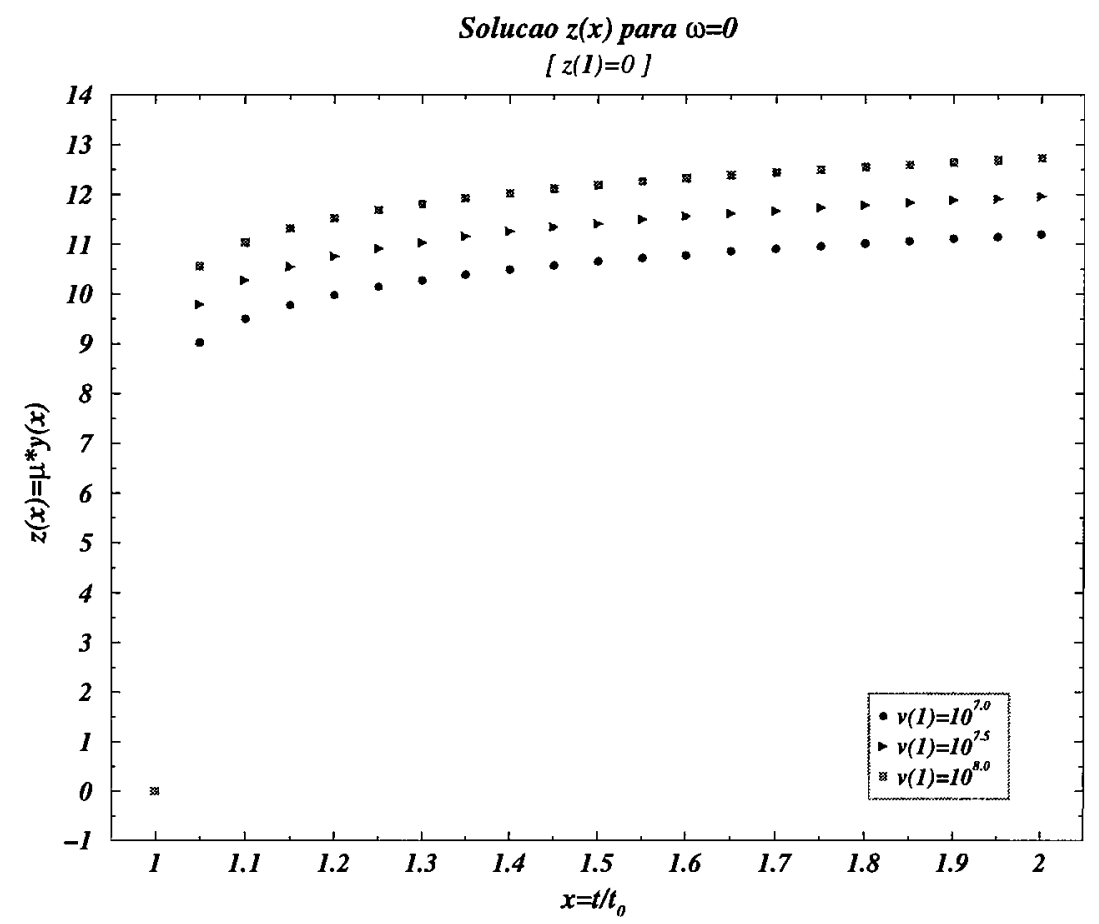

Figura 3.3: O mesmo gráfico que antes com velocidade inicial positiva.

\subsection{Soluções da Equação Geodésica}

Para analisar o comportamento das geodésicas neste modelo, vamos considerar a solução das equações de Einstein com constante cosmológica (3.6), com $\mathcal{C}=0$ [47]

$$
\begin{gathered}
a(t, y)=\left\{\frac{1}{2}\left(1+\frac{\kappa_{(5)}^{2} \rho_{b}^{2}}{6 \rho_{B}}\right)+\frac{1}{2}\left(1-\frac{\kappa_{(5)}^{2} \rho_{b}^{2}}{6 \rho_{B}}\right) \cosh (\mu y)-\frac{\kappa_{(5)} \rho_{b}}{\sqrt{-6 \rho_{B}}} \sinh (\mu|y|)\right\}^{\frac{1}{2}} a_{0}(t) \\
n(t, y)=\frac{\dot{a}(t, y)}{\dot{a}_{0}(t)}
\end{gathered}
$$

com $a_{0}$ e $\mu$ dados anteriormente.

A escolha das constantes aparecendo nesta solução serão feitas de acordo com a discussão na última seção do trabalho de Binétruy et. al. [47], isto é, $\rho_{b}$ é dado por (3.9), $\rho_{B}$ dado de acordo com o ajuste fino (3.11)

$$
\rho_{B}=-\frac{\kappa_{(5)}^{2} \rho_{\Lambda}^{2}}{6}
$$

lembrando que a constante de acoplamento $\kappa_{(5)}$, a constante de Newton $G_{(5)}$, e a massa de Planck $M_{(5)}$ em 5 dimensões estão relacionadas por

$$
\kappa_{(5)}^{2}=8 \pi G_{(5)}=M_{(5)}^{-3}
$$




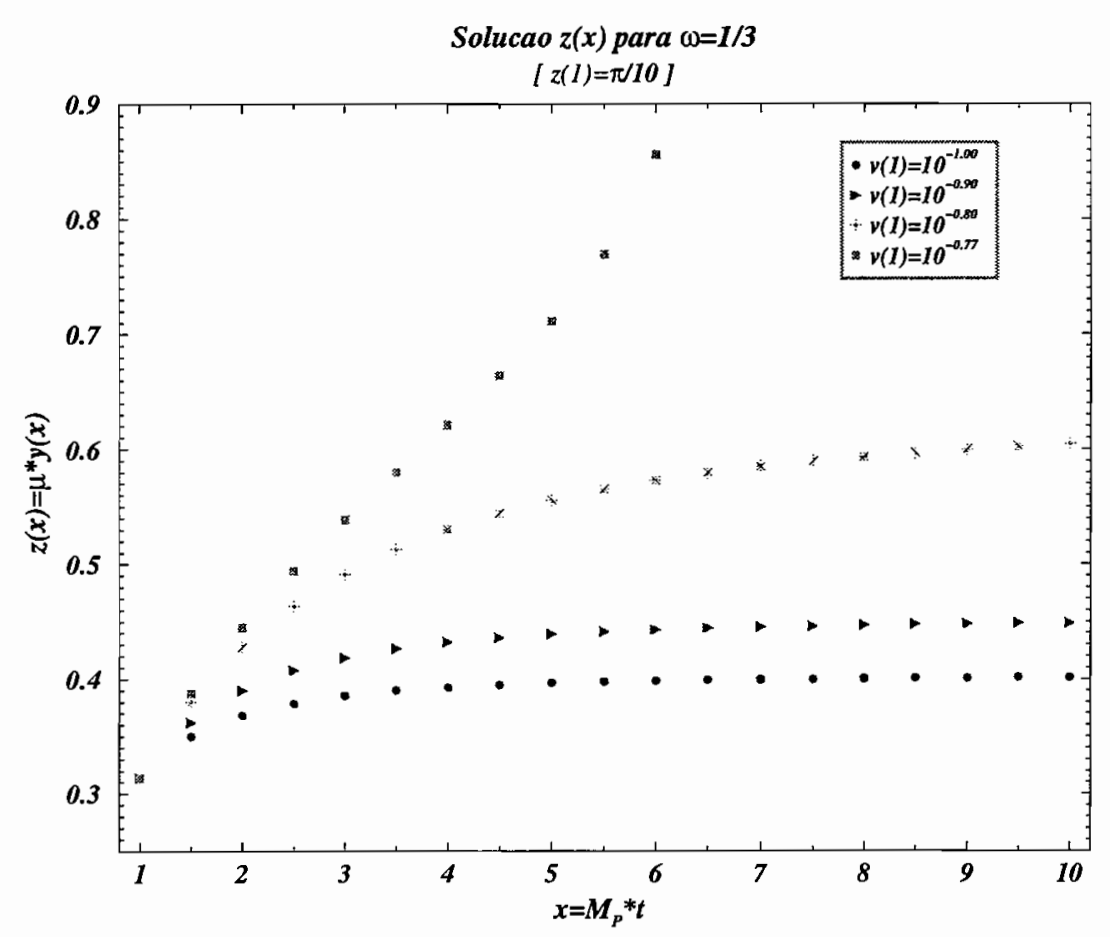

Figura 3.4: Gráfico para $y(1) \sim 0.3 \ell_{P}$ no caso de domínio da radiaçãn. Note o patamar seguido pelo caso de baixa velocidade inicial.

Neste ponto, todas as constantes estão definidas em termos da massa de Planck e nossa discussão da evolução de sinais gravitacionais pode ser estabelecida.

As soluções à equação geodésica (2.35) seguintes [33] foram achadas usando um programa de resolução numérica (método de Runge-Kutta de quarta ordem) em MAPLE (veja apêndice A). Consideramos os casos de domínio de matéria $(\omega=0)$ e de domínio de radiação $(\omega=1 / 3)$.

No caso de domínio da matéria, $\omega=0$, experimentamos usar diferentes condições iniciais. Em geral, preferimos começar com $y \neq 0$ para evitar qualquer solução espúria na equação diferencial, que também é singular. Assim, supomos que $y$ começa na ordem do comprimento de Planck. As Figs. 3.1 a 3.3 mostram alguns resultados. Escolhemos plotar a função adimensional $z(x)=\mu y(x)$, onde $\mu$ corresponde a duas unidades de massa de Planck $M_{P}$ e $x=t / t_{0}$, sendo $t_{0}$ a idade atual do universo.

Cada gráfico contém um conjunto de curvas correspondentes a três velocidades típicas, cujos valores são mostrados na legenda em cada gráfico, produzindo comportamentos similares. Nas figuras 3.1 e 3.2, usamos velocidades iniciais negativas e, independentemente do ponto inicial $y$ escolhido, a curva decai e escapa para nunca retornar à mesma brana. No caso de velocidades iniciais positivas, a Fig. 3.3 mostra três curvas das quais podemos notar que quanto maior for a velocidade inicial, mais longe da brana o objeto viaja. 


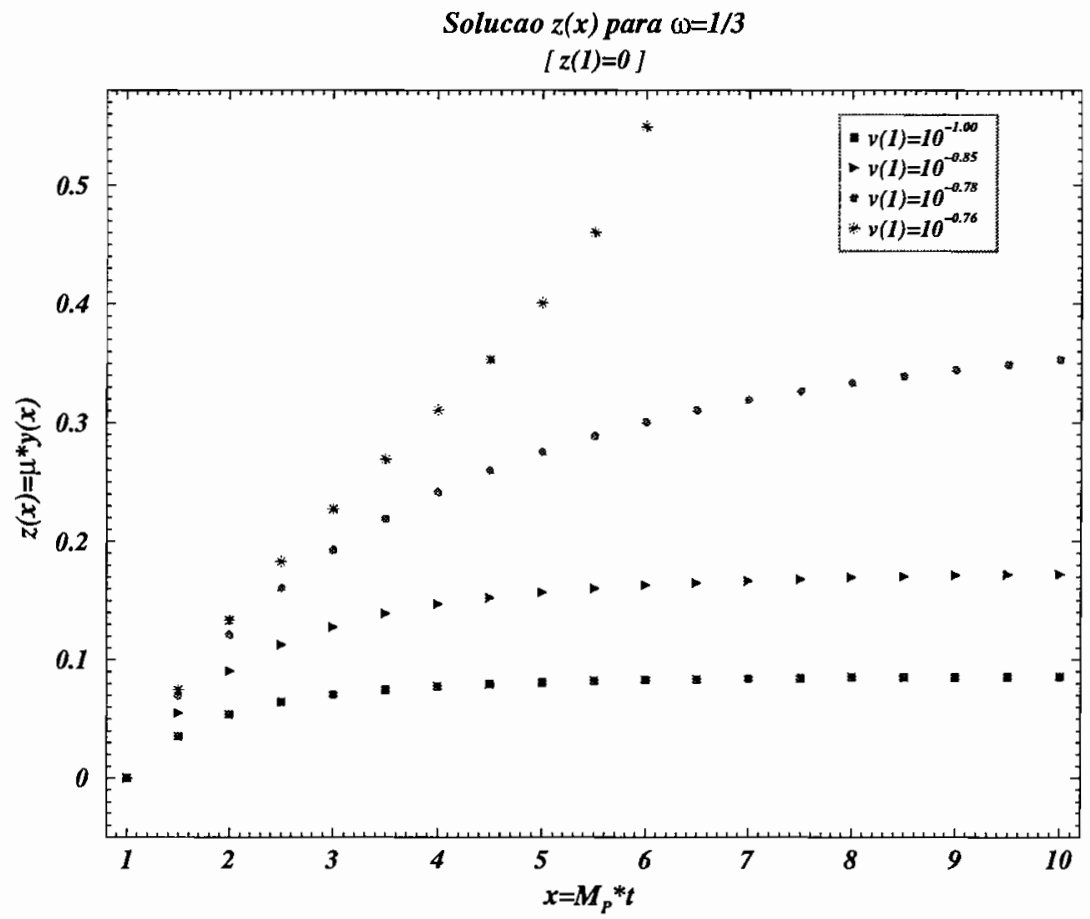

Figura 3.5: O mesmo gráfico com posição inicial zero com respeito à brana.

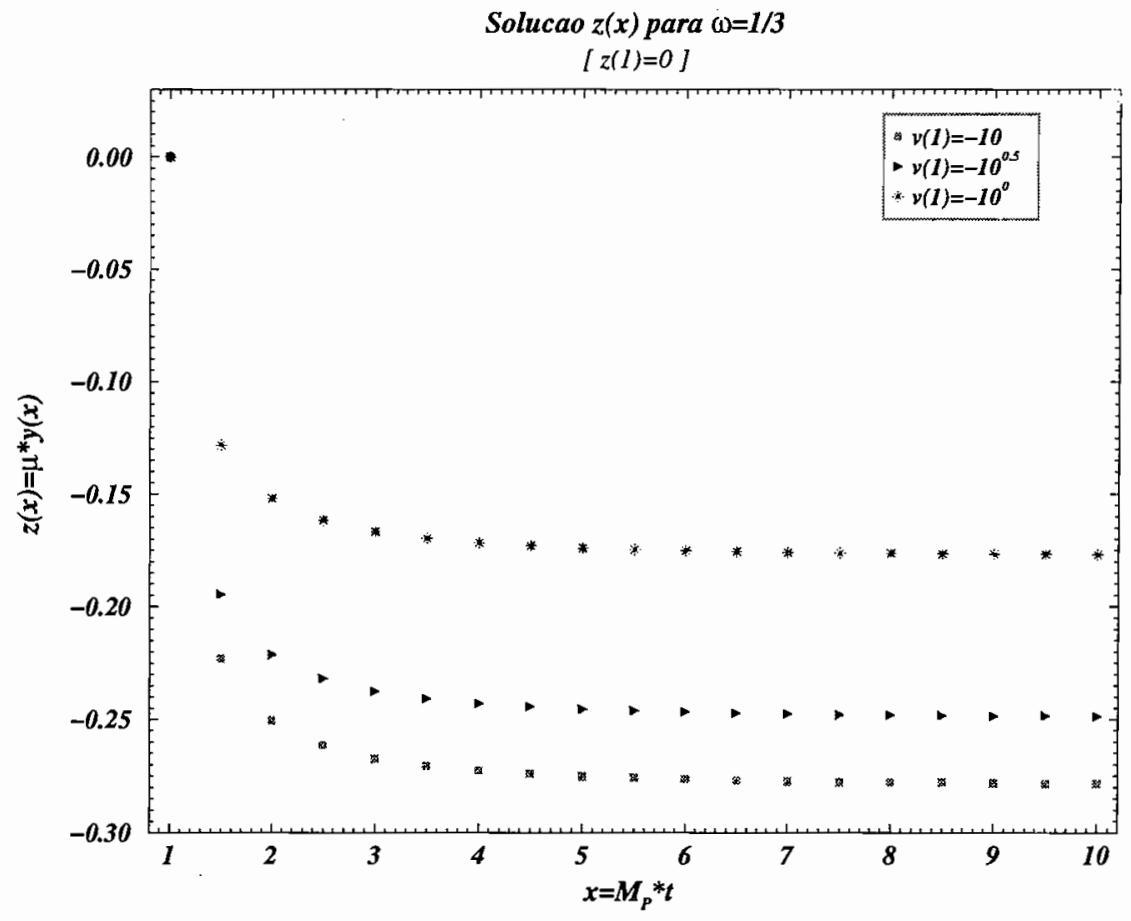

Figura 3.6: O mesmo gráfico que antes com velocidade inicial negativa. 
Resumindo, estes gráficos mostram que nenhuma trajetória retorna à brana depois de desacoplar-se dela. Após esta divisão, algumas trajetórias escapam rapidamente, enquanto que outras ficam quase paralelas à brana por um tempo indefinido.

Assim, acreditamos, baseados nestes resultados, que o caminho mais curto está dentro da brana, sendo aquele seguido pela luz. Porém, existe ainda espaço para outras trajetórias devido ao caráter extremamente complicado da equação diferencial envolvida no problema. Tratamos de pesquisar além se novas estruturas podem aparecer nestas equações diferenciais, mas não as achamos. O fato é que nenhum atalho foi encontrado para este modelo.

$\mathrm{Na}$ era de domínio da radiação, as equações são um pouco mais complicadas. Neste caso, as soluções são mostradas nas Fig. 3.4 a 3.6. Novamente, plotamos a função adimensional $z(x)$, onde $x=M_{P} t$.

As Fig. 3.4 e 3.5 mostram um comportamento de patamar para baixas velocidades iniciais positivas; porém, existe uma velocidade limite para a qual a curva desacopla e escapa ao infinito. A Fig. 3.6 mostra as curvas para três velocidades iniciais negativas. Novamente, a onda gravitacional tenta seguir a brana desde uma distância que depende do valor da velocidade inicial como havíamos visto no caso do domínio da matéria.

Assim, na era de domínio da radiação, $\omega=\frac{1}{2}$, muitas soluções tenciem a se manter perto da brana original, mas nunca voltam. O significado não é conhecido e, de novo, nenhum atalho foi achado.

Portanto, chegamos à conclusão que a cosmologia presente é ainda simples, estando inclusa na larga classe de cosmologias sem atalhos. Nossa pesquisa deve agora encaminhar-se a cosmologias que apresentem novas estruturas, como buracos negros no bulk $[24,23]$. 


\section{Capítulo 4}

\section{Atalhos Gravitacionais em 6 Dimensões}

Neste capítulo vamos descrever os modelos cosmológicos de branas em 6 dimensões que serão usados nos próximos capítulos. Começamos nosso estudo com um modelo simples de uma brana parada em um bulk estático Anti de Sitter, contendo uma singularidade. Seguidamente derivamos a equação geodésica neste modelo que, como veremos mais para frente, pode ser aplicada para qualquer modelo com bulk estático. Esta equação envolve somente a coordenada radial extra, de tal forma que o problema de atalhos 6-dimensional pode ser reduzido a 5 dimensões considerando os coeficientes respetivos das métricas correspondentes. Em seguida, consideramos os modelos de Chamblin e Reall que descrevem paredes de domínio dinâmicas. Posteriormente, generalizamos o modelo de brana estática AdS ao caso de brana dinâmica em espaços-tempos AdS ou dS. Finalmente descrevemos duas aproximações diferentes para a dedução das equações de conservação de energia na brana e para a equação generalizada de Friedmann em 6 dimensões, mostrando a conexão entre modelos de brana estática e dinâmica que, no fim das contas correspondem simplesmente a dois pontos de vista diferentes, a saber, o ponto de vista de um observador na brana e aquele de um observador no bulk.

\subsection{Modelo de Brana Estática}

Como vimos no capítulo 2, a métrica para o bulk foi escrita convenientemente em coordenadas normais gaussianas. Esta escolha não tem restrição física e é muito útil para o ponto de vista da brana; porém, existe uma outra aproximação alternativa para derivar as soluções cosmológicas dadas anteriormente, que recai em um ponto de vista baseado no bulk, e corresponde a uma escolha de coordenadas mais apropriada para o bulk [51]. Esta aproximação conduz à forma simples a seguir, para as soluções 
de Einstein no bulk:

$$
d s^{2}=-f(R) d T^{2}+\frac{d R^{2}}{f(R)}+R^{2} \gamma_{i j} d x^{i} d x^{j}
$$

onde

$$
f(R) \equiv k-\frac{\Lambda}{6} R^{2}-\frac{\mathcal{C}}{R^{2}} .
$$

Esta métrica é conhecida como a métrica Anti de Sitter-Schwarzschild (AdS para $\Lambda<0$, de Sitter para $\Lambda>0$ ). É claro de (4.2) que $\mathcal{C}$ é o análogo da massa de Schwarzschild em 5 dimensões, como foi dito anteriormente.

Esta métrica é manifestamente estática (já que os coeficientes são independentes do tempo), o que quer dizer que as soluções das equações de Einstein têm mais simetrias que aquelas assumidas a priori.

O resultado acima para espaços-tempos vazios em 5 dimensões pode ser visto como uma generalização do teorema de Birkhoff, como é sabido no caso 4-dimensional.

Já que as soluções das equações de Einstein são necessariamente AdS-Schwarzschild, é fácil inferir que as soluções obtidas no sistema coordenado normal gaussiano particular correspondem à mesma geometria, mas escritas em um sistema coordenado mais complicado, como pode ser checado achando a transformação explícita que vai de (4.1) a (2.10) [52].

Se as coordenadas em (4.1) são mais simples para descrever o bulk, não é o caso para a brana mesma. Certamente, enquanto a brana está em repouso (em $y=0)$ no sistema de coordenadas normais gaussianas, sua posição $R$ no novo sistema será em geral dependente do tempo. Isto quer dizer que a brana está se movendo no sistema referencial estático (4.1). Assim, sua evolução cosmológica é simplesmente uma conseqüência do seu movimento no bulk, fenômeno as vezes chamado de cosmologia de miragem [53]. A métrica (4.1) descreve em princípio só um lado da brana. No caso de termos uma simetria de espelho, o espaço-tempo completo é obtido colando duas cópias de uma porção do espaço-tempo AdS-Schwarzschild ao longo da brana.

Vejamos agora, como esta idéia pode ser extendida a 6 dimensões [34].

Consideremos um modelo em 6 dimensões como aquele construído por Kanti et. al. [54]. Procuramos por uma solução da equação de Einstein 6-dimensional no espaço Anti de Sitter, da forma

$$
d s^{2}=-n^{2}(t, y, z) d t^{2}+a^{2}(t, y, z) d \Sigma_{k}^{2}+b^{2}(t, y, z)\left\{d y^{2}+c^{2}(t, y, z) d z^{2}\right\},
$$

onde $d \Sigma_{k}^{2}$ representa a métrica das seções espaciais 3-dimensionais com $k=-1,0,1$, correspondendo aos espaços hiperbólico, plano e elíptico, respectivamente.

O tensor de energia-momento total pode ser decomposto em duas partes, correspondendo ao bulk e à brana, como 


$$
\tilde{T}_{N}^{M}=\breve{T}_{N}^{M(B)}+T_{N}^{M(b)},
$$

onde a contribuição da brana pode ser escrita como

$$
T_{N}^{M(b)}=\frac{\delta\left(z-z_{0}\right)}{b c} \operatorname{diag}(-\rho, p, p, p, \hat{p}, 0) .
$$

As componentes não nulas do tensor de Einstein estão dadas por

$$
\begin{aligned}
& G_{00}=3 \frac{\dot{a}}{a}\left(\frac{\dot{a}}{a}+2 \frac{\dot{b}}{b}+\frac{\dot{c}}{c}\right)+\frac{\dot{b}}{b}\left(\frac{\dot{b}}{b}+\frac{\dot{c}}{c}\right)-\frac{n^{2}}{b^{2}}\left\{\left(3 \frac{\partial_{y}^{2} a}{a}+3 \frac{\left(\partial_{y} a\right)^{2}}{a^{2}}-\frac{\left(\partial_{y} b\right)^{2}}{b^{2}}+\right.\right. \\
& \left.+\frac{\partial_{y}^{2} b}{b}+\frac{\partial_{y}^{2} c}{c}+3 \frac{\partial_{y} a \partial_{y} c}{a c}+\frac{\partial_{y} b \partial_{y} c}{b c}\right)+\frac{1}{c^{2}}\left(3 \frac{\partial_{z}^{2} a}{a}+3 \frac{\left(\partial_{z} a\right)^{2}}{a^{2}}-\right. \\
& \left.\left.-\frac{\left(\partial_{z} b\right)^{2}}{b^{2}}+\frac{\partial_{z}^{2} b}{b}-3 \frac{\partial_{z} a \partial_{z} c}{a c}-\frac{\partial_{z} b \partial_{z} c}{b c}\right)\right\}+6 k \frac{n^{2}}{a^{2}}, \\
& G_{i j}=\frac{a^{2}}{n^{2}}\left\{-2 \frac{\ddot{a}}{a}-2 \frac{\ddot{b}}{b}-\frac{\ddot{c}}{c}-\frac{\dot{a}^{2}}{a^{2}}-\frac{\dot{b}^{2}}{b^{2}}+2 \frac{\dot{a} \dot{n}}{a n}-4 \frac{\dot{a} \dot{b}}{a b}-2 \frac{\dot{a} \dot{c}}{a c}+2 \frac{\dot{b} \dot{n}}{b n}+\frac{\dot{c} \dot{n}}{c n}-\right. \\
& \left.-3 \frac{\dot{b} \dot{c}}{b c}\right\} \gamma_{i j}+\frac{a^{2}}{b^{2}}\left\{\left(2 \frac{\partial_{y}^{2} a}{a}+\frac{\left(\partial_{y} a\right)^{2}}{a^{2}}+\frac{\partial_{y}^{2} b}{b}-\frac{\left(\partial_{y} b\right)^{2}}{b^{2}}+\frac{\partial_{y}^{2} n}{n}+\frac{\partial_{y}^{2} c}{c}+\right.\right. \\
& \left.+2 \frac{\partial_{y} a \partial_{y} n}{a n}+2 \frac{\partial_{y} a \partial_{y} c}{a c}+\frac{\partial_{y} c \partial_{y} n}{c n}+\frac{\partial_{y} b \partial_{y} c}{b c}\right)+\frac{1}{c^{2}}\left(2 \frac{\partial_{z}^{2} a}{a}+\frac{\left(\partial_{z} a\right)^{2}}{a^{2}}+\right. \\
& +\frac{\partial_{z}^{2} b}{b}-\frac{\left(\partial_{z} b\right)^{2}}{b^{2}}+\frac{\partial_{z}^{2} n}{n}+2 \frac{\partial_{z} a \partial_{z} n}{a n}-2 \frac{\partial_{z} a \partial_{z} c}{a c}-\frac{\partial_{z} c \partial_{z} n}{c n}- \\
& \left.\left.-\frac{\partial_{z} b \partial_{z} c}{b c}\right)\right\} \gamma_{i j}-3 k \gamma_{i j} \\
& G_{55}=\left\{-\frac{b^{2}}{n^{2}}\left(\frac{\ddot{b}}{b}+3 \frac{\ddot{a}}{a}+\frac{\ddot{c}}{c}-\frac{\dot{b} \dot{n}}{b n}+3 \frac{\dot{a} \dot{b}}{a b}+2 \frac{\dot{b} \dot{c}}{b c}-3 \frac{\dot{a} \dot{n}}{a n}-\frac{\dot{c} \dot{n}}{c n}+3 \frac{\dot{a} \dot{c}}{a c}+3 \frac{\dot{a}^{2}}{a^{2}}\right)+\right. \\
& +3 \frac{\left(\partial_{y} a\right)^{2}}{a^{2}}+3 \frac{\partial_{y} a \partial_{y} b}{a b}+3 \frac{\partial_{y} a \partial_{y} c}{a c}+3 \frac{\partial_{y} a \partial_{y} n}{a n}+\frac{\partial_{y} b \partial_{y} n}{b n}+\frac{\partial_{y} c \partial_{y} n}{c n}+ \\
& +\frac{1}{c^{2}}\left\{3 \frac{\partial_{z}^{2} a}{a}+3 \frac{\left(\partial_{z} a\right)^{2}}{a^{2}}+\frac{\partial_{z}^{2} n}{n}-\left(3 \frac{\partial_{z} a \partial_{z} b}{a b}+3 \frac{\partial_{z} a \partial_{z} c}{a c}-3 \frac{\partial_{z} a \partial_{z} n}{a n}+\right.\right. \\
& \left.\left.\left.+\frac{\partial_{z} b \partial_{z} n}{b n}+\frac{\partial_{z} c \partial_{z} n}{c n}\right)\right\}-3 k \frac{b^{2}}{a^{2}}\right\} \gamma_{55}, \\
& G_{66}=-\frac{b^{2} c^{2}}{n^{2}}\left(3 \frac{\ddot{a}}{a}+\frac{\ddot{b}}{b}-\frac{\dot{b} \dot{n}}{b n}+3 \frac{\dot{a} \dot{b}}{a b}-3 \frac{\dot{a} \dot{n}}{a n}+3 \frac{\dot{a}^{2}}{a^{2}}\right)+ \\
& +c^{2}\left\{3 \frac{\partial_{y}^{2} a}{a}+\frac{\partial_{y}^{2} n}{n}+3 \frac{\left(\partial_{y} a\right)^{2}}{a^{2}}+3 \frac{\partial_{y} a \partial_{y} n}{a n}-3 \frac{\partial_{y} a \partial_{y} b}{a b}-\frac{\partial_{y} b \partial_{y} n}{b n}\right\}+ \\
& +3 \frac{\left(\partial_{z} a\right)^{2}}{a^{2}}+3 \frac{\partial_{z} a \partial_{z} b}{a b}+3 \frac{\partial_{z} a \partial_{z} n}{a n}+\frac{\partial_{z} b \partial_{z} n}{b n}-6 k \frac{b^{2} c^{2}}{a^{2}},
\end{aligned}
$$




$$
\begin{aligned}
G_{05}= & -3 \frac{\partial_{y} \dot{a}}{a}-\frac{\partial_{y} \dot{b}}{b}-\frac{\partial_{y} \dot{c}}{c}+3 \frac{\dot{a}}{a}\left(\frac{\partial_{y} n}{n}\right)+\frac{\dot{b}}{b}\left(\frac{\partial_{y} n}{n}+3 \frac{\partial_{y} a}{a}+\frac{\partial_{y} b}{b}\right)+ \\
& +\frac{\dot{c}}{c}\left(\frac{\partial_{y} n}{n}-\frac{\partial_{y} b}{b}\right) \\
G_{06}= & -3 \frac{\partial_{z} \dot{a}}{a}-\frac{\partial_{z} \dot{b}}{b}+3 \frac{\dot{a}}{a}\left(\frac{\partial_{z} n}{n}\right)+\frac{\dot{b}}{b}\left(\frac{\partial_{z} n}{n}+3 \frac{\partial_{z} a}{a}+\frac{\partial_{z} b}{b}\right)+ \\
& +\frac{\dot{c}}{c}\left(3 \frac{\partial_{z} a}{a}+\frac{\partial_{z} b}{b}\right) \\
G_{56}= & -\frac{\partial_{z} \partial_{y} n}{n}-3 \frac{\partial_{z} \partial_{y} a}{a}+\frac{\partial_{z} b \partial_{y} n}{b n}+3 \frac{\partial_{y} a \partial_{z} b}{a b}+\frac{\partial_{y} b \partial_{z} n}{b n}+\frac{\partial_{y} c \partial_{z} n}{c n}+ \\
& +3 \frac{\partial_{z} a \partial_{y} b}{a b}+3 \frac{\partial_{z} a \partial_{y} c}{a c}
\end{aligned}
$$

Para se ter uma geometria bem definida, a métrica deve ser contínua através da brana; porém, suas derivadas com respeito a $z$ podem ser descontínuas na posição da brana, gerando uma função delta de Dirac nas derivadas segundas da métrica com respeito a $z$ [10], como vimos no caso 5-dimensional. Estes termos contendo a função delta devem ser casados com as componentes do tensor de energia-momento (4.5) para satisfazer as equações de Einstein.

Assim, usando (4.6), (4.7) e (4.8) obtemos as seguintes condições de DarmoisIsrael,

$$
\begin{aligned}
\frac{\left[\partial_{z} a\right]}{a_{0} b_{0} c_{0}} & =-\frac{\kappa_{(6)}^{2}}{4}(p-\hat{p}+\rho), \\
\frac{\left[\partial_{z} b\right]}{b_{0}^{2} c_{0}} & =-\frac{\kappa_{(6)}^{2}}{4}\{\rho-3(p-\hat{p})\} \\
\frac{\left[\partial_{z} n\right]}{b_{0} c_{0} n_{0}} & =\frac{\kappa_{(6)}^{2}}{4}\{\hat{p}+3(p+\rho)\} .
\end{aligned}
$$

Uma métrica da forma (4.3) satisfazendo as equações de Einstein em 6 dimensões está dada por

$$
d s^{2}=-h(z) d t^{2}+\frac{z^{2}}{l^{2}} d \Sigma_{k}^{2}+h^{-1}(z) d z^{2}
$$

onde

$$
d \Sigma_{k}^{2}=\frac{d r^{2}}{1-k r^{2}}+r^{2} d \Omega_{(2)}^{2}+\left(1-k r^{2}\right) d y^{2}
$$

e

$$
\begin{array}{cl}
h(z)=k+\frac{z^{2}}{l^{2}}-\frac{M}{z^{3}} & , \quad \text { para o bulk AdS-Schwarzschild }, \\
h(z)=k+\frac{z^{2}}{l^{2}}-\frac{M}{z^{3}}+\frac{Q^{2}}{z^{6}} \quad, \quad \text { para o bulk AdS-Reissner-Nordström }
\end{array}
$$

com $l^{-2} \propto-\Lambda$ ( $\Lambda$ sendo a constante cosmológica), que descreve um buraco negro no bulk, situado em $z=0$. 
Desta forma podemos identificar

$$
\begin{aligned}
n(z) & =\sqrt{h(z)} \\
a(z) & =b(z)=\frac{z}{l}, \quad \mathrm{e} \\
b(z) c(z) & =\sqrt{h^{-1}(z)} .
\end{aligned}
$$

Seguindo [24], achamos uma outra solução usando a simetria $Z_{2}$, invertendo o espaço com respeito à posição da brana. Isto é, considerando uma métrica da forma

$$
d s^{2}=-A^{2}(z) d t^{2}+B^{2}(z) d \Sigma_{(4)}^{2}+C^{2}(z) d z^{2}
$$

e a brana estando definida em $z=z_{0}$, existe uma solução dada por

$$
\begin{array}{ccc}
A(z), B(z), C(z) & , \quad \text { para } \quad z \leq z_{0} \\
A\left(z_{0}^{2} / z\right), B\left(z_{0}^{2} / z\right), C\left(z_{0}^{2} / z\right) \frac{z_{0}^{2}}{z^{2}} \quad, \quad \text { para } \quad z \geq z_{0} .
\end{array}
$$

A simetria $Z_{2}$ corresponde a $z \rightarrow z_{0}^{2} / z$.

A brana estática deve obedecer às condições de Darmois-Israel (4.13), que para a métrica (4.14) são escritas como

$$
\begin{aligned}
\frac{\left[\partial_{z} a\right]}{a_{0}^{2} c_{0}} & =-\frac{\kappa_{(6)}^{2}}{4} \rho, \\
\frac{\left[\partial_{z} n\right]}{a_{0} c_{0} n_{0}} & =\frac{\kappa_{(6)}^{2}}{4}(4 p+3 \rho),
\end{aligned}
$$

onde

$$
\begin{aligned}
& {\left[\partial_{z} a\right]=-\frac{2}{l}} \\
& {\left[\partial_{z} n\right]=-\frac{h^{\prime}\left(z_{0}\right)}{\sqrt{h\left(z_{0}\right)}} .}
\end{aligned}
$$

\subsection{Equação para os Atalhos}

Consideremos a métrica (4.3) com $k=0$ e chamando $d(z)=b(z) c(z)$

$$
d s^{2}=-n^{2}(z) d t^{2}+a^{2}(z) f^{2}(r) d r^{2}+b^{2}(z) d y^{2}+d^{2}(z) d z^{2},
$$

onde a trajetória do gráviton, parametrizada por $z=z(t)$, é definida fazendo (4.23) igual a zero. Portanto,

$$
\int_{r_{0}}^{r} f\left(r^{\prime}\right) d r^{\prime}=\int_{t_{0}}^{t} \frac{\sqrt{n^{2}(z)-b^{2}(z) \dot{y}^{2}-d^{2}(z) \dot{z}^{2}}}{a(z)} d t \equiv \int_{t_{0}}^{t} \mathcal{L}[y(t), \dot{y}(t), z(t), \dot{z}(t) ; t] d t
$$


que naturalmente define uma densidade de lagrangiana. As equações de EulerLagrange de $\mathcal{L}$ definem a trajetória do gráviton. Neste caso,

$$
\begin{aligned}
\frac{\partial \mathcal{L}}{\partial y}= & 0 \\
\frac{\partial \mathcal{L}}{\partial \dot{y}}= & -\frac{1}{a}\left(n^{2}-b^{2} \dot{y}^{2}-d^{2} \dot{z}^{2}\right)^{-1 / 2} b^{2} \dot{y} \\
\frac{\partial \mathcal{L}}{\partial z}= & -\frac{a^{\prime}}{a^{2}} \sqrt{n^{2}-b^{2} \dot{y}^{2}-d^{2} \dot{z}^{2}}+ \\
& +\frac{1}{a}\left(n^{2}-b^{2} \dot{y}^{2}-d^{2} \dot{z}^{2}\right)^{-1 / 2}\left(n n^{\prime}-b b^{\prime} \dot{y}^{2}-d d^{\prime} \dot{z}^{2}\right) \\
\frac{\partial \mathcal{L}}{\partial \dot{z}}= & -\frac{1}{a}\left(n^{2}-b^{2} \dot{y}^{2}-d^{2} \dot{z}^{2}\right)^{-1 / 2} d^{2} \dot{z}
\end{aligned}
$$

Primeiramente vamos trabalhar com $y$ constante para checar a possibilidade de que (4.23) admita atalhos. Assim, a equação de Euler-Lagrange resultante será

$$
\begin{aligned}
\ddot{z}+\left(\frac{\dot{a}}{a} \frac{d^{2}}{n^{2}}-\frac{d \dot{d}}{n^{2}}\right) \dot{z}^{3}+\left(\frac{a^{\prime}}{a}-2 \frac{n^{\prime}}{n}+\frac{d^{\prime}}{d}\right) \dot{z}^{2} & +\left(2 \frac{\dot{d}}{d}-\frac{\dot{a}}{a}-\frac{\dot{n}}{n}\right) \dot{z}+ \\
+ & \left(\frac{n n^{\prime}}{d^{2}}-\frac{a^{\prime}}{a} \frac{n^{2}}{d^{2}}\right)=0
\end{aligned}
$$

que no caso de $n=n(z), a=a(z)$, e $d=d(z)$, se torna simples, mas está longe de ser trivial,

$$
\ddot{z}+\left(\frac{a^{\prime}}{a}-2 \frac{n^{\prime}}{n}+\frac{d^{\prime}}{d}\right) \dot{z}^{2}+\left(\frac{n n^{\prime}}{d^{2}}-\frac{a^{\prime}}{a} \frac{n^{2}}{d^{2}}\right)=0 .
$$

Lembremos que para $z \leq z_{0}$, temos $a=z / l, n=\sqrt{h(z)}$, e $d=1 / \sqrt{h(z)}$. Enquanto que para $z \geq z_{0}$, devemos usar a simetria $Z_{2}$ mostrada em (4.20).

Note que este caso é equivalente a considerar o problema em 5 dimensões com a métrica mostrada em [24].

O caso mais geral inclui uma dependência de $y$ na trajetória do gráviton; porém, como mostraremos a seguir, esta dependência torna-se supérflua e não afeta a equação de $z$, já que (4.30) é independentemente satisfeita. Esta conclusão não é surpreendente se notamos que a métrica (4.23) é independente de $y$.

As duas equações de Euler-Lagrange considerando uma dependência em $y$ são então dadas por

$$
\begin{array}{r}
\left(n^{2}-d^{2} \dot{z}^{2}\right) \ddot{y}+\dot{z}\left\{\left(-\frac{a^{\prime}}{a}+2 \frac{b^{\prime}}{b}\right)\left(n^{2}-d^{2} \dot{z}^{2}\right)-n n^{\prime}+\right. \\
\left.+d d^{\prime} \dot{z}^{2}+d^{2} \ddot{z}\right\} \dot{y}+b^{2}\left(\frac{a^{\prime}}{a}-\frac{b^{\prime}}{b}\right) \dot{z} \dot{y}^{3}=0
\end{array}
$$


e

$$
\begin{aligned}
\left(n^{2}-b^{2} \dot{y}^{2}\right) \ddot{z}+\{ & \left.\left(\frac{a^{\prime}}{a}+\frac{d^{\prime}}{d}\right)\left(n^{2}-b^{2} \dot{y}^{2}\right)-2 n n^{\prime}+2 b b^{\prime} \dot{y}^{2}\right\} \dot{z}^{2}+\left(b^{2} \dot{y} \ddot{y}\right) \dot{z}+ \\
& +\left\{-\frac{a^{\prime}}{a d^{2}}\left(n^{2}-b^{2} \dot{y}^{2}\right)+\frac{n n^{\prime}-b b^{\prime} \dot{y}^{2}}{d^{2}}\right\}\left(n^{2}-b^{2} \dot{y}^{2}\right)=0
\end{aligned}
$$

É claro que o caso $\dot{y}=0$ é uma solução deste conjunto de equações quando, ao mesmo tempo, $z$ obedece (4.30).

Substituindo os valores de $n, a$ e $d$ dados em (4.18), este conjunto de equações pode ser manipulado conduzindo a

$$
\begin{aligned}
\frac{z \dot{z} \dot{y}}{h(z)} F_{z}+\left(h(z)-\frac{\dot{z}^{2}}{h(z)}\right) F_{y} & =0 \\
\left(1-\frac{z^{2} \dot{y}^{2}}{h(z)}\right) F_{z}+\frac{z \dot{z} \dot{y}}{h(z)} F_{y} & =0
\end{aligned}
$$

onde

$$
\begin{aligned}
& F_{y}=\ddot{y}+\dot{z}\left(\frac{2}{z}-\frac{h^{\prime}(z)}{h(z)}\right) \dot{y} \\
& F_{z}=\frac{\ddot{z}}{z}+\frac{\dot{z}^{2}}{z^{2}}\left(1-\frac{3}{2} z \frac{h^{\prime}(z)}{h(z)}\right)+\frac{h(z)}{z}\left(\frac{h^{\prime}(z)}{2}-\frac{h(z)}{z}\right)
\end{aligned}
$$

Já que o determinante do conjunto (4.33) é diferente de zero, as soluções de (4.31) e (4.32) devem satisfazer $F_{y}=0$ e $F_{z}=0$ independentemente. Ainda mais, notemos que $F_{y}=0$ e $F_{z}=0$ são as equações de geodésicas nulas para $y$ e $z$, respectivamente, obtidas de

$$
\ddot{x}^{\alpha}+\Gamma_{\mu \nu}^{\alpha} \dot{x}^{\mu} \dot{x}^{\nu}=\lambda \dot{x}^{\alpha} .
$$

Assim, uma curva nula é extrema, se e somente se, é uma geodésica nula. Então, como (4.34) é a mesma que (4.30), nosso problema é reduzido ao caso prévio com $y$ constante descrito por (4.30).

Para os casos com $k \neq 0$, podemos também considerar (4.30) como a equação de atalhos se assumirmos a existência de uma simetria em $y$ no nosso problema. Desta forma nosso modelo representa uma generalização de [24].

\subsection{Modelos de Chamblin e Reall}

Uma parede de domínio em um espaço-tempo $D$-dimensional pode ser definida como um objeto extendido com $D-2$ dimensões espaciais, que divide o espaço-tempo em 
diferentes domínios. Usaremos o termo "parede de domínio" para nos referirmos a qualquer $(D-2)$-brana se movendo em $D$ dimensões.

O modelo seguinte descreve a gravitação de Einstein com um campo escalar (dílaton) no bulk e uma parede de domínio acoplada ao dílaton do bulk.

O cenário está descrito pela ação gravitacional em um bulk $D$-dimensional com um campo escalar no bulk, um potencial na parede de domínio, e um termo de GibbonsHawking [55]:

$$
S=\int_{b u l k} d^{D} x \sqrt{-g}\left(\frac{1}{2} R-\frac{1}{2}(\partial \phi)^{2}-V(\phi)\right)-\int_{p d} d^{D-1} x \sqrt{-h}([K]+\hat{V}(\phi)),
$$

onde $\phi$ é o dílaton do bulk, $K$ é a curvatura extrínseca, $V(\phi)$ e $\hat{V}(\phi)$ são os potenciais do bulk e da parede de domínio, respetivamente, e $g$ e $h$ denotam as métricas do bulk e da parede de domínio, respetivamente. Note que os colchetes em $K$ denotam uma soma sobre os dois lados da parede. Os potenciais aqui são considerados do tipo Liouville:

$$
\begin{aligned}
& V(\phi)=V_{0} e^{\beta \phi} \\
& \hat{V}(\phi)=\hat{V}_{0} e^{\alpha \phi} .
\end{aligned}
$$

Consideramos a métrica do bulk como sendo estática e invariante sob rotação, sendo dada por

$$
d s^{2}=-U(r) d t^{2}+U(r)^{-1} d r^{2}+R(r)^{2} d \Omega_{k}^{2},
$$

onde $d \Omega_{k}^{2}$ é o elemento de linha em um espaço $D-2$-dimensional de curvatura constante dependendo no parâmetro $k$, e $r$ representa a coordenada extra não compactificada. Supomos que tal métrica tenha simetria de espelho $Z_{2}$ com respeito à parede de domínio. Tal simetria será usada para impor as condições de Darmois-Israel [46]. De fato, a variação da ação total (4.36), incluindo o termo Gibbons-Hawking, conduz diretamente às condições de Darmois-Israel que, em vista da simetria $Z_{2}$, produzem

$$
K_{M N}=-\frac{1}{2(D-2)} \hat{V}(\phi) h_{M N}
$$

A curvatura extrínseca pode ser calculada como

$$
K_{M N}=h_{M}^{P} h_{N}^{Q} \nabla_{P} n_{Q},
$$

onde a normal unitária, que aponta a $r<r(t)$ (sendo $r=r(t)$ a posição da parede de domínio no bulk), é

$$
n_{M}=\frac{1}{\sqrt{U-\frac{\dot{r}^{2}}{U}}}(\dot{r},-1,0 \ldots, 0) .
$$

Aqui o ponto indica derivada com respeito ao tempo do bulk $t$. 
Assim, as componentes da curvatura extrínseca relevantes são

$$
\begin{aligned}
& K_{00}=\frac{1}{\dot{r}} \frac{d}{d t}\left(\frac{U}{\sqrt{U-\frac{\dot{r}^{2}}{U}}}\right), \\
& K_{i j}=-U \frac{R^{\prime}}{R} \frac{1}{\sqrt{U-\frac{\dot{r}^{2}}{U}}} h_{i j} .
\end{aligned}
$$

Substituindo nas condições de Darmois-Israel (4.40), a componente $(i j)$ pode ser escrita como

$$
\frac{R^{\prime}}{R}=\frac{\hat{V}(\phi)}{2(D-2) U} \sqrt{U-\frac{\dot{r}^{2}}{U}},
$$

que, combinada com a componente (00), dá

$$
\left(\frac{R^{\prime}}{R}\right)^{-1}\left(\frac{R^{\prime}}{R}\right)^{\prime}=\frac{\hat{V}^{\prime}(\phi)}{\hat{V}(\phi)}-\frac{R^{\prime}}{R} .
$$

Aqui a linha denota derivada com respeito à coordenada radial extra $r$.

A equação de movimento para o dílaton pode ser obtida variando a ação (4.36),

$$
\frac{d \phi}{d R}=-\frac{D-2}{R} \frac{1}{\hat{V}} \frac{d \hat{V}}{d \phi}
$$

e junto com (4.46), formam um sistema que pode ser simultaneamente resolvido com o Ansatz (4.39), levando-nos a [56]

$$
\begin{aligned}
& \phi(r)=\phi_{\star}-\frac{\alpha(D-2)}{\alpha^{2}(D-2)+1} \log r \\
& R(r)=\left[\alpha^{2}(D-2)+1\right] C \hat{V}_{0} e^{\alpha \phi_{\star}} r^{\frac{1}{\left[\alpha^{2}(D-2)+1\right]}}
\end{aligned}
$$

onde $\phi_{\star}$ e $C$ são constantes de integração arbitrárias.

O movimento da parede de domínio é governada pela componente $(i j)$ das condições de Darmois-Israel (4.45). Essa equação pode ser escrita em termos do tempo próprio da brana $\tau$, como

$$
\frac{1}{2}\left(\frac{d R}{d \tau}\right)^{2}+F(R)=0 .
$$

A métrica induzida na parede de domínio é Friedmann-Robertson-Walker e (4.50) descreve a evolução do fator de escala $R(\tau)$. Esta equação é a mesma que aquela para uma partícula de massa unidade e energia nula, rolando em um potencial $F(R)$, dado por

$$
F(R)=\frac{1}{2} U R^{\prime 2}-\frac{1}{8(D-2)^{2}} \hat{V}^{2} R^{2}
$$


Note que a solução existe somente quando $F(R) \leq 0$.

Das equações de Einstein é possível distinguir três tipos de soluções para a métrica do bulk.

\subsubsection{Soluções Tipo-I}

As soluções tipo-I tem $\alpha=\beta=0$, tal que os potenciais tornam-se constantes cosmológicas e de (4.48) é fácil ver que o dílaton também vira uma constante. Assim, a métrica admite um reescalonamento tal que

$$
d s^{2}=-U(R) d t^{2}+U(R)^{-1} d R^{2}+R^{2} d \Omega_{k}^{2},
$$

com

$$
U(R)=k-2 M R^{-(D-3)}-\frac{2 V_{0}}{(D-1)(D-2)} R^{2},
$$

onde $M$ é uma constante. Se $M=0$, isto é simplesmente a métrica do espaço-tempo de De Sitter, Minkowski ou Anti de Sitter. Para $M \neq 0, U(R)$ é desenhado na Fig.4.1.
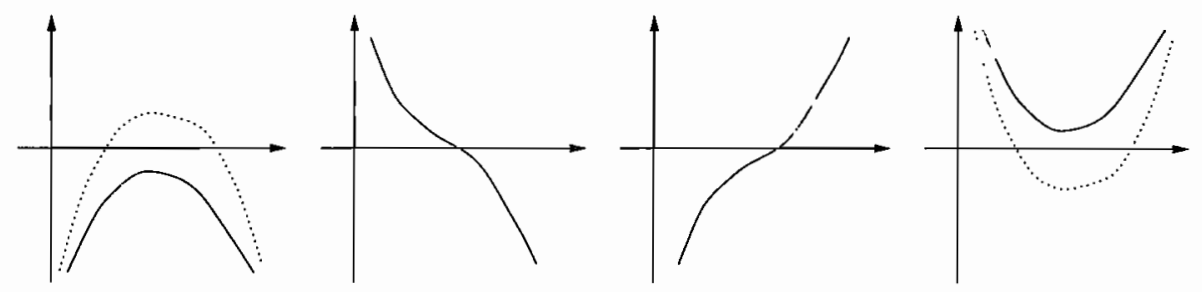

Figura 4.1: $U(R)$ para $V_{0}>0, M>0 ; V_{0}>0, M<0 ; V_{0}<0, M>0$; e $V_{0}<0, M<$ 0 , respetivamente. As linhas pontilhadas indicam um comportamento alternativo (modificado de [56]).

Como vemos, existem 4 casos, a saber, $V_{0}>0, M>0 ; V_{0}>0, M<0 ; V_{0}<$ $0, M>0$; e $V_{0}<0, M<0$. O primeiro caso para $k=1$ descreve um bulk dSSchwarzschild, enquanto que para $k \neq 1$ existe uma singularidade cosmológica em um espaço assintoticamente dS. O segundo caso descreve uma singularidade nua com horizonte cosmológico depois do qual a métrica se aproxima à métrica dS. O terceiro caso descreve buracos negros topológicos em um bulk AdS, em especial, note que para $k=1$ a solução corresponde a um bulk AdS-Schwarzschild. O ultimo caso descreve um buraco negro topológico para $k=-1$ e uma singularidade nua para $k \neq-1$, em um bulk assintoticamente AdS. 

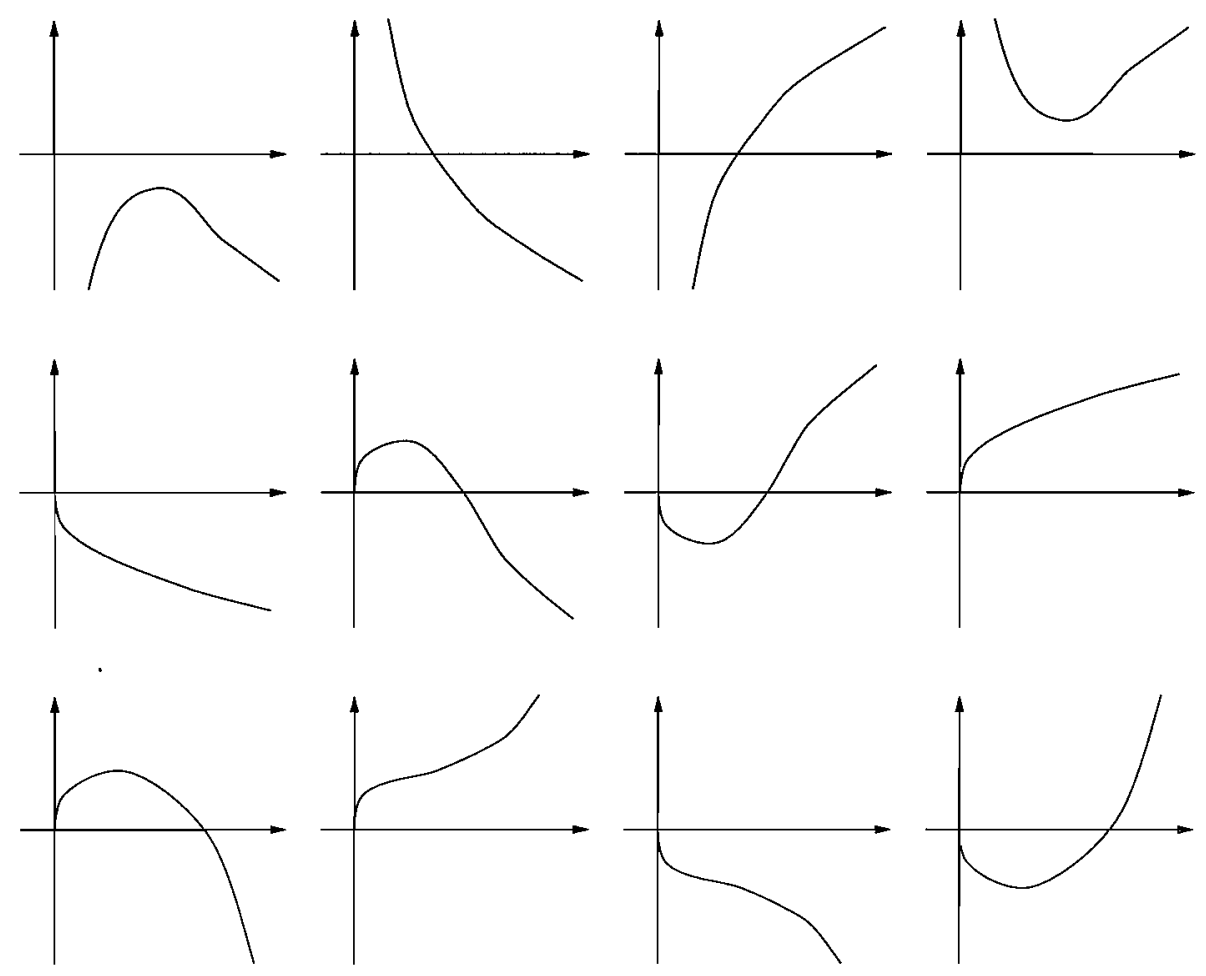

Figura 4.2: $U(R)$ para $V_{0}>0, M>0 ; V_{0}>0, M<0 ; V_{0}<0, M>0$; e $V_{0}<0, M<$ 0 (colunas), respetivamente. Os comportamentos variam também para $b^{2}<D-3$, $D-3<b^{2}<D-1$, e $b^{2}>D-1$ (fileiras), respetivamente (modificado de [56]).

\subsubsection{Soluções Tipo-II}

Estas soluções estão definidas por $\alpha=\beta / 2$ e $k=0$. A métrica está determinada por

$$
U(r)=\left(1+b^{2}\right)^{2} r^{\frac{2}{\left(1+b^{2}\right)}}\left(-2 M r^{-\frac{\left(D-1-b^{2}\right)}{\left(1+b^{2}\right)}}-\frac{2 \Lambda}{\left(D-1-b^{2}\right)}\right),
$$

e o fator de escala é

$$
R(r)=r^{\frac{1}{\left(1+b^{2}\right)}}
$$

onde a "constante cosmológica efetiva" e o parâmetro $b$ estão dados por

$$
\begin{aligned}
\Lambda & =\frac{V_{0} e^{2 b \phi_{0}}}{D-2}, \\
b & =\frac{1}{2} \beta \sqrt{D-2} .
\end{aligned}
$$

A forma para a função $U(r)$ é desenhada na Fig.4.2. Todas as soluções tipo-II são singulares em $r=0$. Algumas possuem horizontes do tipo buraco negro ou horizontes cosmológicos. 


\subsubsection{Soluções Tipo-III}

Estas soluções possuem $\alpha=2 / \beta(D-2)$. A métrica é dada por

$$
U(r)=\left(1+b^{2}\right)^{2} r^{\frac{2}{\left(1+b^{2}\right)}}\left(-2 M r^{\frac{-\left[1+b^{2}(D-3)\right]}{\left(1+b^{2}\right)}}-\frac{2 \Lambda}{\left[1+b^{2}(D-3)\right]}\right)
$$

e o fator de escala é

$$
R(r)=\gamma r^{b^{2} /\left(1+b^{2}\right)}
$$

onde o parâmetro $\gamma$ é

$$
\gamma=\left(\frac{(D-3)}{2 k \Lambda\left(1-b^{2}\right)}\right)^{1 / 2}
$$

Os valores de $\Lambda$ e $b$ são os mesmos que aqueles das soluções tipo-II.
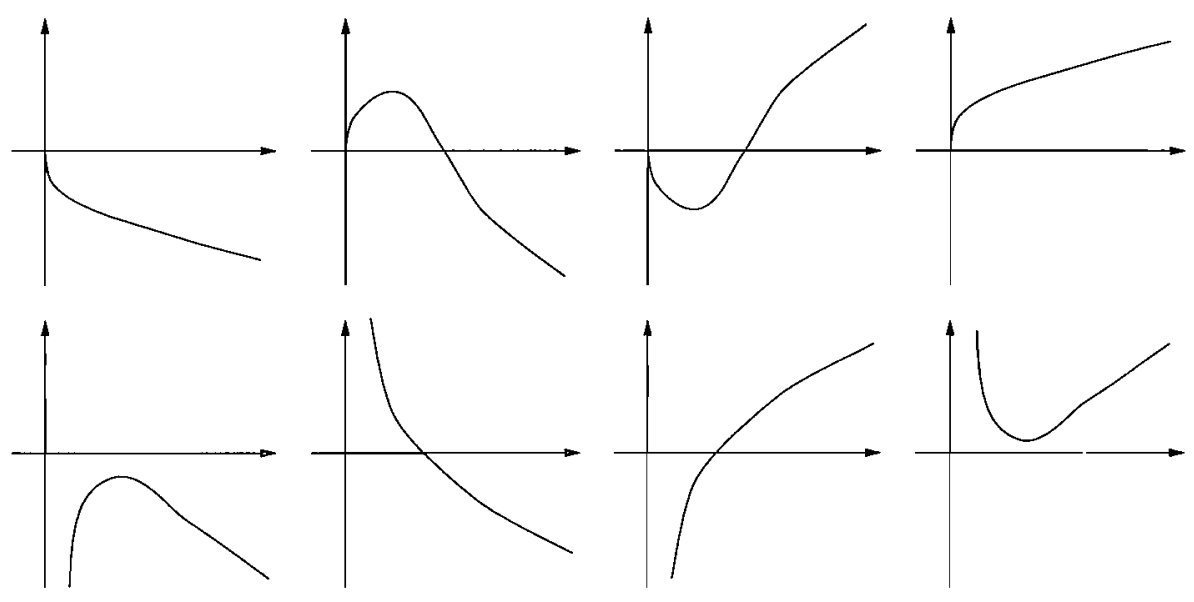

Figura 4.3: $U(R)$ para $V_{0}>0, M>0 ; V_{0}>0, M<0 ; V_{0}<0, M>0$; e $V_{0}<0, M<$ 0 (colunas), respetivamente. Os comportamentos variam também para $b^{2}<1 /(D-3)$ e $b^{2}>1 /(D-3)$ (fileiras), respetivamente (modificado de [56]).

As possíveis formas para $U(r)$ são mostradas na Fig.4.3. Note que o fato de $V_{0}$ ser negativo determina que $r$ seja uma coordenada tipo-espaço, o que nos será útil no capítulo 6. Em particular, se além de $V_{0}<0$ temos também $M>0$, as soluções são de tipo buraco negro.

\subsection{Modelo de Brana Dinâmica}

Vamos generalizar agora o modelo mostrado na seção 4.1 para o caso de uma brana se movendo em um fundo estático. Consideramos o modelo em 6 dimensões descrito 
pela métrica (4.3)

$$
d s^{2}=-n^{2}(t, y, z) d t^{2}+a^{2}(t, y, z) d \Sigma_{k}^{2}+b^{2}(t, y, z) d y^{2}+d^{2}(t, y, z) d z^{2},
$$

onde, como antes, $d \Sigma_{k}^{2}$ representa a métrica das seções espaciais 3-dimensionais com $k=-1,0,1$ correspondendo a um espaço hiperbólico, plano e elíptico, respetivamente.

O conteúdo de matéria na brana está diretamente relacionado ao salto do tensor de curvatura extrínseca através da brana $[56,57]$. Esta relação foi derivada no caso de uma brana estática na seção 4.1 [34]. Aqui generalizamos nosso resultado deduzindo as condições de junção desde um ponto de vista diferente àquele de distribuições. Neste caso, as condições de Darmois-Israel irão incluir o caso de uma brana se movendo com respeito ao sistema coordenado, cuja posição em qualquer tempo do bulk $t$ será denotada por

$$
z=\mathcal{R}(t)
$$

O tensor de curvatura extrínseca na brana é dado por

$$
K_{M N}=h_{M}^{L} \nabla_{L} \tilde{n}_{N},
$$

onde $\tilde{n}^{A}$ é um campo vetorial unitário normal à brana $\mathrm{e}$

$$
h_{M N}=g_{M N}-\tilde{n}_{M} \tilde{n}_{N}
$$

é a métrica induzida na brana.

Para calcular as componentes de $\tilde{n}^{A}$, usamos as relações

$$
g_{M N} \tilde{n}^{M} \tilde{n}^{N}=1 \quad, \quad g_{M N} \tilde{n}^{M} u^{N}=0,
$$

onde introduzimos o vetor velocidade unitário correspondente à brana,

$$
u^{A}=\left\{\frac{d t}{d \tau}, 0,0,0,0, \frac{d z}{d \tau}\right\}
$$

A relação entre $d t$ (tempo do bulk) e $d \tau$ (tempo da brana) pode ser achado da métrica induzida,

$$
\begin{aligned}
d s_{\text {induzida }}^{2} & =-\left[n^{2}(t, \mathcal{R}(t))-d^{2}(t, \mathcal{R}(t)) \dot{z}^{2}\right] d t^{2}+a^{2}(t, \mathcal{R}(t)) d \Sigma_{(4)}^{2} \\
& =-d \tau^{2}+a^{2}(\tau) d \Sigma_{(4)}^{2}
\end{aligned}
$$

onde o ponto indica derivada com respeito ao tempo do bulk $t$. Desta forma, obtemos

$$
d \tau=n(t, \mathcal{R}(t)) \sqrt{1-\frac{d^{2}(t, \mathcal{R}(t))}{n^{2}(t, \mathcal{R}(t))} \dot{\mathcal{R}}^{2}} d t \equiv n \gamma^{-1} d t
$$


Assim, (4.66) pode ser escrito como

$$
u^{A}=\frac{\gamma}{n}\{1,0,0,0,0, \dot{\mathcal{R}}\},
$$

e de (4.65) podemos facilmente obter

$$
\tilde{n}^{A}=\left\{\frac{d \dot{\mathcal{R}}}{n^{2} \sqrt{1-\frac{d^{2}}{n^{2}} \dot{\mathcal{R}}^{2}}}, 0,0,0,0, \frac{1}{d \sqrt{1-\frac{d^{2}}{n^{2}} \dot{\mathcal{R}}^{2}}}\right\}
$$

Agora podemos calcular as componentes do tensor de curvatura extrínseca, substituindo (4.70) em (4.63). As componentes não nulas são

$$
\begin{aligned}
K_{0}^{0}= & \frac{d}{n^{2}}\left(1-\frac{d^{2}(t, \mathcal{R}(t))}{n^{2}(t, \mathcal{R}(t))}\right)^{-5 / 2} \times \\
& \times\left\{\ddot{\mathcal{R}}+\frac{n n^{\prime}}{d^{2}}-\dot{\mathcal{R}}\left(\frac{\dot{n}}{n}-2 \frac{\dot{d}}{d}\right)-\dot{\mathcal{R}}^{2}\left(2 \frac{n^{\prime}}{n}-\frac{d^{\prime}}{d}\right)-\dot{\mathcal{R}}^{3} \frac{d \dot{d}}{n^{2}}\right\}, \\
K_{0}^{6}= & \dot{\mathcal{R}} K_{0}^{0}, \quad K_{6}^{0}=-\frac{d^{2}}{n^{2}} \dot{\mathcal{R}} k_{0}^{0} \\
K_{i}^{j}= & \frac{1}{d}\left(1-\frac{d^{2}(t, \mathcal{R}(t))}{n^{2}(t, \mathcal{R}(t))}\right)^{-1 / 2}\left\{\frac{a^{\prime}}{a}+\frac{\dot{a}}{a} \frac{d^{2}}{n^{2}} \dot{\mathcal{R}}\right\} \delta_{i}^{j} \\
K_{5}^{5}= & \frac{1}{d}\left(1-\frac{d^{2}(t, \mathcal{R}(t))}{n^{2}(t, \mathcal{R}(t))}\right)^{-1 / 2}\left\{\frac{b^{\prime}}{b}+\frac{\dot{b}}{b} \frac{d^{2}}{n^{2}} \dot{\mathcal{R}}\right\} \\
K_{6}^{6}= & -\frac{d^{2}}{n^{2}} \dot{\mathcal{R}}^{2} K_{0}^{0},
\end{aligned}
$$

onde todos os coeficientes tomam seu valor na brana.

\section{Condições de Darmois-Israel}

O tensor de energia-momento na brana localizada em $z_{0}$ pode ser escrito como

$$
T_{M N}^{(b)}=\frac{\delta\left(z-z_{0}\right)}{d}\left\{(\rho+p) u_{M} u_{N}+p h_{M N}\right\}
$$

Definimos também um tensor $\hat{T}_{A B}$ como

$$
\hat{T}_{A B} \equiv T_{A B}-\frac{1}{4} T h_{A B}
$$

As condições de junção de Darmois-Israel [46] são dadas por

$$
\left[K_{\mu \nu}\right]=-\kappa_{(6)}^{2} \hat{T}_{\mu \nu}
$$


onde os colchetes indicam o salto através da brana e $K_{\mu \nu}=e_{\mu}^{A} e_{\nu}^{B} K_{A B}$, onde $e_{\mu}^{A}$ formam uma base do espaço vetorial tangente ao volume da brana.

As componentes não nulas de (4.77) são dadas por

$$
\begin{aligned}
& \hat{T}_{0}^{0}=-\frac{\gamma^{2}}{d}\left\{\frac{3 \rho+4 p}{4}\right\}, \\
& \hat{T}_{0}^{6}=\dot{\mathcal{R}} \hat{T}_{0}^{0}, \quad \hat{T}_{6}^{0}=-\frac{d^{2}}{n^{2}} \dot{\mathcal{R}} \hat{T}_{0}^{0}, \\
& \hat{T}_{i}^{j}=\frac{\rho}{4 d} \delta_{i}^{j}, \quad \hat{T}_{5}^{5}=\hat{T}_{i}^{i}, \\
& \hat{T}_{6}^{6}=-\frac{d^{2}}{n^{2}} \dot{\mathcal{R}}^{2} \hat{T}_{0}^{0} .
\end{aligned}
$$

O lado esquerdo de (4.78) pode ser calculado levando em conta a simetria de espelho através da brana,

$$
\left[K_{\mu \nu}\right]=K_{\mu \nu}\left(t, \mathcal{R}(t)^{+}\right)-K_{\mu \nu}\left(t, \mathcal{R}(t)^{-}\right)=-2 K_{\mu \nu}(t, \mathcal{R}(t)) .
$$

Neste ponto, é conveniente escolher uma métrica específica para o bulk da forma (4.61), que satisfaça as equações de Einstein em 6 dimensões. Esta métrica está dada por (4.14)

$$
d s^{2}=-h(z) d t^{2}+a^{2}(z) d \Sigma_{k}^{2}+h^{-1}(z) d z^{2},
$$

onde

e

$$
\begin{gathered}
a(z)=\frac{z}{l}, \\
d \Sigma_{k}^{2}=\frac{d r^{2}}{1-k r^{2}}+r^{2} d \Omega_{(2)}^{2}+\left(1-k r^{2}\right) d y^{2}
\end{gathered}
$$

$$
h(z)=k+\frac{z^{2}}{l^{2}}-\frac{M}{z^{3}}+\frac{Q^{2}}{z^{6}}
$$

com $l^{-2} \propto-\Lambda$ ( $\Lambda$ sendo a constante cosmológica, que pode ser positiva ou negativa) e $M$ e $Q^{2}$ são constantes. Devemos notar que $y$ é uma coordenada compactificada.

Esta métrica contém uma singularidade localizada em $z=0$. A métrica acima é valida nas partes $z<\mathcal{R}(t)$ de superfícies de $t$ constante, e sua reflexão, pela simetria de orbifold $Z_{2}$, é valida nas partes $z>\mathcal{R}(t)$. Se $M=0$ e $Q^{2}=0$, então (4.84) é simplesmente a métrica do espaço-tempo de Sitter ou Anti de Sitter de acordo com o sinal de $l^{2}$.

Com este Ansatz, as condições de Darmois-Israel (4.78) se reduzem a somente duas equações,

$$
\ddot{\mathcal{R}}+\frac{1}{2} \frac{h^{\prime}}{h^{3}} \dot{\mathcal{R}}^{4}-3 \frac{h^{\prime}}{h} \dot{\mathcal{R}}^{2}+\frac{1}{2} h h^{\prime}=-\kappa_{(6)}^{2}\left(\frac{3 \rho+4 p}{8}\right) h^{2}\left(1-\frac{\dot{\mathcal{R}}^{2}}{h^{2}}\right)^{3 / 2}
$$




$$
\frac{a^{\prime}}{a}+\frac{\dot{\mathcal{R}}}{h^{2}} \frac{\dot{a}}{a}=\kappa_{(6)}^{2} \frac{\rho}{8}\left(1-\frac{\dot{\mathcal{R}}^{2}}{h^{2}}\right)^{1 / 2}
$$

onde novamente todos os coeficientes da métrica devem ser calculados na brana. O sistema (4.88) descreve a dinâmica não linear completa da brana imersa no bulk estático (4.84). No capítulo 7 estudaremos todas as soluções possíveis deste sistema no contexto cosmológico.

\subsection{Equações de Friedmann}

Vamos apresentar duas aproximações equivalentes da dedução das equações de Friedmann e da equação de conservação de energia na brana em modelos 6-dimensionais.

\subsubsection{Dedução por Distribuições}

Primeiramente, vamos achar a equação de conservação de energia na brana. Para isto, tomemos o salto da componente (06) das equações de Einstein, usando a Eq.(4.11)

$$
\begin{aligned}
{\left[G_{06}\right]=} & -\frac{3}{a_{0}}\left[\partial_{z} \dot{a}\right]-\frac{1}{b_{0}}\left[\partial_{z} \dot{b}\right]+3 \frac{\dot{a}_{0}}{a_{0} n_{0}}\left[\partial_{z} n\right]+\frac{\dot{b}_{0}}{b_{0}}\left\{\frac{\left[\partial_{z} n\right]}{n_{0}}+3 \frac{\left[\partial_{z} a\right]}{a_{0}}+\frac{\left[\partial_{z} \dot{b}\right]}{b_{0}}\right\}+ \\
& +\frac{\dot{c}_{0}}{c_{0}}\left\{3 \frac{\left[\partial_{z} a\right]}{a_{0}}+\frac{\left[\partial_{z} b\right]}{b_{0}}\right\}=0 .
\end{aligned}
$$

Usando as condições de junção (4.13) e as derivadas temporais correspondentes, dadas por

$$
\begin{aligned}
\partial_{t}\left(\frac{\left[\partial_{z} a\right]}{a_{0} b_{0} c_{0}}\right) & =-\frac{\kappa_{(6)}^{2}}{4}(\dot{p}-\dot{\hat{p}}+\dot{\rho}), \\
\partial_{t}\left(\frac{\left[\partial_{z} b\right]}{b_{0}^{2} c_{0}}\right) & =-\frac{\kappa_{(6)}^{2}}{4}\{\dot{\rho}-3(\dot{p}-\dot{\hat{p}})\}
\end{aligned}
$$

obtemos

$$
\dot{\rho}+3(p+\rho) \frac{\dot{a}_{0}}{a_{0}}+(\hat{p}+\rho) \frac{\dot{b}_{0}}{b_{0}}=0,
$$

que é a equação de conservação de energia numa brana imersa em um bulk 6-dimensional. Note que esta equação também pode ser encontrada usando as identidades de Bianchi $\nabla_{M} G_{N}^{M}=0$. No caso especial $a=b$, esta equação torna-se

$$
\dot{\rho}+4(p+\rho) \frac{\dot{a}_{0}}{a_{0}}=0 .
$$


Para achar as equações de Friedmann, tomemos o salto da componente (66) das equações de Einstein, usando a Eq.(4.9)

$$
3 \frac{\left[\left(\partial_{z} a\right)^{2}\right]}{a_{0}^{2}}+3 \frac{\left[\partial_{z} a \partial_{z} b\right]}{a_{0} b_{0}}+3 \frac{\left[\partial_{z} a \partial_{z} n\right]}{a_{0} n_{0}}+\frac{\left[\partial_{z} b \partial_{z} n\right]}{b_{0} n_{0}}=0 .
$$

Usamos o fato que

$$
\left[\partial_{z} f \partial_{z} g\right]=\# \partial_{z} f \#\left[\partial_{z} g\right]+\left[\partial_{z} f\right] \# \partial_{z} g \#
$$

onde

$$
\# f \#=\frac{f\left(0^{+}\right)+f\left(0^{-}\right)}{2},
$$

é o valor médio da função $f$ através de $y=0$. E chegamos na seguinte equação

$$
\frac{\# \partial_{z} a \#}{a_{0}} p=\frac{1}{3} \rho \frac{\# \partial_{z} n \#}{n_{0}}-\frac{1}{3} \hat{p} \frac{\# \partial_{z} b \#}{b_{0}} \text {. }
$$

Tomemos agora o valor médio da componente (66) das equações de Einstein, usando a Eq.(4.9)

$$
\begin{gathered}
-\frac{b_{0}^{2} c_{0}^{2}}{n_{0}^{2}}\left(3 \frac{\ddot{a}_{0}}{a_{0}}+\frac{\ddot{b}_{0}}{b_{0}}-\frac{\dot{b}_{0} \dot{n}_{0}}{b_{0} n_{0}}+3 \frac{\dot{a}_{0} \dot{b}_{0}}{a_{0} b_{0}}-3 \frac{\dot{a}_{0} \dot{n}_{0}}{a_{0} n_{0}}+3 \frac{\dot{a}_{0}^{2}}{a_{0}^{2}}\right)+3 c_{0}^{2} \frac{\partial_{y}^{2} a_{0}}{a_{0}}+ \\
+c_{0} \frac{\partial_{y}^{2} n_{0}}{n_{0}}+3 c_{0}^{2} \frac{\left(\partial_{y} a_{0}\right)^{2}}{a_{0}^{2}}+c_{0}^{2}\left(3 \frac{\partial_{y} a_{0} \partial_{y} n_{0}}{a_{0} n_{0}}-3 \frac{\partial_{y} a_{0} \partial_{y} b_{0}}{a_{0} b_{0}}-\frac{\left.\partial_{y} b_{0} \partial_{y} n_{0}\right)}{b_{0} n_{0}}\right)= \\
=-3 \frac{\#\left(\partial_{z} a\right)^{2} \#}{a_{0}^{2}}-3 \frac{\# \partial_{z} a \partial_{z} b \#}{a_{0} b_{0}}-3 \frac{\# \partial_{z} a \partial_{z} n \#}{a_{0} n_{0}}-\frac{\# \partial_{z} b \partial_{z} n \#}{b_{0} n_{0}}+ \\
+6 k \frac{b_{0}^{2} c_{0}^{2}}{a_{0}^{2}}+\kappa_{(6)}^{2} \breve{T}_{66} .
\end{gathered}
$$

Analogamente à equação (4.95), temos que

$$
\# \partial_{z} f \partial_{z} g \#=\# \partial_{z} f \# \# \partial_{z} g \#+\frac{1}{4}\left[\partial_{z} f\right]\left[\partial_{z} g\right] .
$$

Assim, usando (4.98), as equações de junção, (4.96) e o fato de que $\# \partial_{z} a \#=$ $\# \partial_{z} b \#=0$ (devido à simetria $\left.Z_{2}\right)$, chegamos a

$$
\begin{gathered}
\left(\frac{\ddot{a}_{0}}{a_{0}}+\frac{1}{3} \frac{\ddot{b}_{0}}{b_{0}}+\frac{\dot{a}_{0} \dot{b}_{0}}{a_{0} b_{0}}+\frac{\dot{a}_{0}^{2}}{a_{0}^{2}}\right)-\frac{1}{b_{0}^{2}}\left(\frac{\partial_{y}^{2} a_{0}}{a_{0}}+\frac{\left(\partial_{y} a_{0}\right)^{2}}{a_{0}^{2}}-\frac{\partial_{y} a_{0} \partial_{y} b_{0}}{a_{0} b_{0}}\right)= \\
=-\frac{\kappa_{(6)}^{2}}{32}\left\{\rho\left(\rho+2 p+\frac{2}{3} \hat{p}\right)+(p-\hat{p})^{2}\right\}-2 \frac{k}{a_{0}^{2}}-\frac{\kappa_{(6)}^{2}}{3 b_{0}^{2} c_{0}^{2}} \breve{T}_{66},
\end{gathered}
$$

que é a equação de Friedmann generalizada em 6 dimensões. Sem perda de generalidade, seguindo [10], escolhemos o tempo cosmológico na brana tal que $n_{0}=1$. 


\subsubsection{Dedução das Condições de Darmois-Israel}

As equações de Friedmann previamente obtidas (4.99) podem ser reescritas no Ansatz $a(\tau)=b(\tau) \equiv \mathcal{R}$, como

$$
2 \frac{\ddot{\mathcal{R}}}{\mathcal{R}}+3\left(\frac{\dot{\mathcal{R}}}{\mathcal{R}}\right)^{2}=-3 \frac{\kappa_{(6)}^{4}}{64}\left\{\rho\left(\rho+\frac{8}{3} p\right)\right\}-\frac{\kappa_{(6)}^{2}}{2 d_{0}^{2}} \breve{T}_{66}-3 \frac{k}{\mathcal{R}^{2}},
$$

onde o ponto indica derivada com respeito ao tempo da brana $\tau$.

Desde o ponto de vista da brana podemos achar equações de junção analogamente ao caso do ponto de vista do bulk deduzido na seção 4.4 .

Vamos definir um vetor normal à brana

$$
n_{A}=(\dot{\mathcal{R}}, 0,0,0,0,-\dot{t}) \text {. }
$$

As componentes relevantes do tensor de curvatura extrínseca na brana são dadas por

$$
\begin{aligned}
K_{00} & =\frac{\partial x^{A}}{\partial \tau} \nabla_{\tau} n_{A}=\frac{\partial x^{A}}{\partial \tau}\left\{\partial_{\tau} n_{A}-\frac{\partial^{C}}{\partial \tau} \Gamma_{C A}^{B} n_{B}\right\} \\
K_{i j} & =-\Gamma_{i j}^{A} n_{A},
\end{aligned}
$$

onde os símbolos de Christoffel não nulos para á métrica estática (4.3), são dados por

$$
\begin{gathered}
\Gamma_{06}^{0}=\Gamma_{60}^{0}=\frac{n^{\prime}}{n}, \\
\Gamma_{j 6}^{i}=\Gamma_{6 j}^{i}=\frac{a^{\prime}}{a}, \\
\Gamma_{56}^{5}=\Gamma_{65}^{5}=\frac{b^{\prime}}{b}, \\
\Gamma_{00}^{6}=\frac{n n^{\prime}}{b^{2} c^{2}}, \quad \Gamma_{i j}^{6}=-\frac{a a^{\prime}}{b^{2} c^{2}} \delta_{i j}, \\
\Gamma_{55}^{6}=-\frac{b^{\prime}}{b c^{2}}, \quad \Gamma_{66}^{6}=\frac{b^{\prime}}{b}+\frac{c^{\prime}}{c} .
\end{gathered}
$$

A condição de unitariedade do vetor normal implica em

$$
-h \dot{\mathcal{R}}^{2}+\frac{\dot{t}^{2}}{h}=1
$$

As componentes do tensor de energia-momento na brana têm a forma de um fluido perfeito:

$$
T_{\mu \nu}=(\rho+p) u_{\mu} u_{\nu}+p h_{\mu \nu}
$$

onde o vetor velocidade está definido na brana como

$$
u^{\mu}=(1,0,0,0,0)
$$


e a métrica induzida na 4-brana, $h_{\mu \nu}$, tem a forma (4.67).

As condições de Darmois-Israel na brana tomam a forma

$$
\left[K_{\nu}^{\mu}\right]=-\kappa_{(6)}^{2} \hat{T}_{\nu}^{\mu},
$$

onde

$$
\hat{T}_{\nu}^{\mu}=T_{\nu}^{\mu}-\frac{1}{4} T h_{\nu}^{\mu} .
$$

Assim, as componentes $(\tau \tau)$ e $(y y)$ das condições de Darmois-Israel produzem

$$
\begin{aligned}
\frac{-h^{\prime}-2 \ddot{\mathcal{R}}}{\sqrt{h+\dot{\mathcal{R}}^{2}}} & =-\kappa_{(6)}^{2}\left(\frac{3}{4} \rho+p\right), \\
-2 \frac{\sqrt{h+\dot{\mathcal{R}}^{2}}}{\mathcal{R}} & =\frac{\kappa_{(6)}^{2}}{4} \rho .
\end{aligned}
$$

A Eq. (4.109) produz imediatamente a equação de Friedmann:

$$
\frac{\dot{\mathcal{R}}^{2}}{\mathcal{R}^{2}}=\frac{\kappa_{(6)}^{4}}{64} \rho^{2}-\frac{h}{\mathcal{R}^{2}} .
$$

Derivando (4.109) com respeito ao tempo e substituindo sucessivamente (4.109) e (4.108) obtemos a equação de conservação de energia na brara:

$$
\dot{\rho}+4(\rho+p) \frac{\dot{\mathcal{R}}}{\mathcal{R}}=0,
$$

que é a mesma equação obtida das identidades de Bianchi e do salto da componente (06) das equações de Einstein (4.93).

Finalmente, comparemos nosso resultado (4.110) com a Eq. (4.99) escrita na forma (4.100). Combinando (4.108) e (4.109) temos

$$
2 \frac{\ddot{\mathcal{R}}}{\mathcal{R}}+3\left(\frac{\dot{\mathcal{R}}}{\mathcal{R}}\right)^{2}=-3 \frac{\kappa_{(6)}^{4}}{64} \rho^{2}-\frac{\kappa_{(6)}^{4}}{8} \rho p-\frac{h^{\prime}}{\mathcal{R}}-3 \frac{h}{\mathcal{R}^{2}} .
$$

Substituindo a forma explícita de $h$ dada em (4.16), por exemplo, teremos

$$
2 \frac{\ddot{\mathcal{R}}}{\mathcal{R}}+3\left(\frac{\dot{\mathcal{R}}}{\mathcal{R}}\right)^{2}=-3 \frac{\kappa_{(6)}^{4}}{64} \rho^{2}-\frac{\kappa_{(6)}^{4}}{8} \rho p-3 \frac{k}{\mathcal{R}^{2}}-\frac{5}{l^{2}} .
$$

Comparando com (4.100), vemos que

$$
\begin{array}{r}
\kappa_{(6)}^{2} \breve{T}_{6}^{6}=\frac{\kappa_{(6)}^{2}}{d_{0}^{2}} \breve{T}_{66}=\frac{10}{l^{2}} \\
\Rightarrow l^{-2}=-\frac{\kappa_{(6)}^{2}}{10} \Lambda
\end{array}
$$


onde $\breve{T}_{6}^{6}=-\Lambda$ é a constante cosmológica do bulk.

Portanto, provamos a equivalência de ambas deduções. Em geral, a aproximação por distribuições é mais útil nos casos onde a brana está parada seja em um bulk estático ou dinâmico; enquanto a aproximação pelas condições de Darmois-Israel se mostra mais geral quando consideramos branas dinâmicas. 


\section{Capítulo 5}

\section{Atalhos no Modelo de Brana Estática}

Neste capítulo vamos considerar o modelo de branas em 6 dimensões com fatores de warp asimétricos para o tempo e as duas coordenadas extras y e $z$ [34], descrito na seção 4.1. Resolvemos o conjunto de equações para o caminho mais curto mostrado no capítulo anterior para bulks tipo Anti de Sitter-Schwarzschild e Anti de SitterReissner-Nordström. Em ambos casos derivamos um conjunto de condições para a existência de atalhos em bulks com singularidades protegidas e mostramos alguns exemplos de atalhos obtidos sob estas condições. Finalmente discutimos algumas conseqüências.

\subsection{O Bulk AdS-Schwarzschild}

Como vimos no capítulo anterior, o cenário AdS-Schwarzschild em 6 dimensões é descrito pela métrica

$$
d s^{2}=-h(z) d t^{2}+\frac{z^{2}}{l^{2}} d \Sigma_{k}^{2}+h^{-1}(z) d z^{2}
$$

onde

e

$$
d \Sigma_{k}^{2}=\frac{d r^{2}}{1-k r^{2}}+r^{2} d \Omega_{(2)}^{2}+\left(1-k r^{2}\right) d y^{2}
$$

$$
h(z)=k+\frac{z^{2}}{l^{2}}-\frac{M}{z^{3}}
$$

com $l^{-2} \propto-\Lambda$ ( $\Lambda$ sendo a constante cosmológica negativa no bulk).

Das condições de Darmois-Israel (4.21), junto com (4.22), temos

$$
\frac{h}{z_{0}^{2}}=\frac{\kappa_{(6)}^{4} \rho^{2}}{64}
$$




$$
\frac{h^{\prime}}{2 z_{0}}=-\frac{\kappa_{(6)}^{4} \rho^{2}}{64}(4 \omega+3),
$$

e podemos obter a "massa" do buraco negro $M$ como função da densidade de energia na brana $\rho$, enquanto $\rho$ é fixada por um ajuste fino:

$$
\begin{aligned}
\frac{M}{z_{0}^{5}} & =\frac{2}{5} \frac{k}{z_{0}^{2}}-(\omega+1) \frac{\kappa_{(6)}^{4} \rho^{2}}{40}, \\
\frac{\kappa_{(6)}^{4} \rho^{2}}{64} & =-\frac{3 k}{z_{0}^{2}(8 \omega+3)}-\frac{5}{(8 \omega+3) l^{2}},
\end{aligned}
$$

onde $\omega=p / \rho$.

Como vimos no capítulo prévio, os atalhos em 6 dimensões são determinados da Eq. (4.30). Também devemos lembrar que a brana está estática em $z=z_{0}$. Se um atalho existe, deve haver um tempo $t=v$ na trajetória do gráviton, no qual $\dot{z}(v)=0$ e $\ddot{z}(v) \geq 0$. Assim, (4.30) calculada neste ponto será

$$
\ddot{z}(v)+h\left(z_{v}\right)\left(\frac{h^{\prime}\left(z_{v}\right)}{2}-\frac{h\left(z_{v}\right)}{z_{v}}\right)=0 .
$$

É obvio que este mínimo deve estar entre a brana e o horizonte de eventos $z_{h}$, se um horizonte existe. De outra maneir э, não há ponto de retorno na trajetória desde que o gráviton não pode voltar depois de passar através do horizonte de eventos. Portanto, $h\left(z_{v}\right)>0$.

Assim, de (5.8) precisamos

$$
F\left(z_{v}\right)=\frac{h^{\prime}\left(z_{v}\right)}{2}-\frac{h\left(z_{v}\right)}{z_{v}} \leq 0 \quad \text { para } \quad z_{h}<z_{v}<z_{0} .
$$

Para visualizar melhor a discussão seguinte, ilustramos a situação na Fig.5.1 para o caso $k=1$. Usando (4.16), isto implica

$$
F(z)=\frac{5}{2} \frac{M}{z^{4}}-\frac{k}{z}
$$

Esta equação tem um zero em $z=z_{f} \neq 0$ para $k \neq 0$

$$
z_{f}^{3}=\frac{5}{2 k} M \text {. }
$$

Assim, para os casos $k=0$ ou $k=-1$, não existe raiz positiva. Já que a massa, $M$, é positiva, $F(z)>0$ em qualquer lugar, prevenindo a coexistência de atalhos e horizontes.

De outro lado, para $k=1$, existe uma única raiz positiva e real, que deve satisfazer $z_{f}<z_{0}$, para ter atalhos. Isto é,

$$
\frac{5 M}{2 z_{0}^{3}}-1<0 .
$$




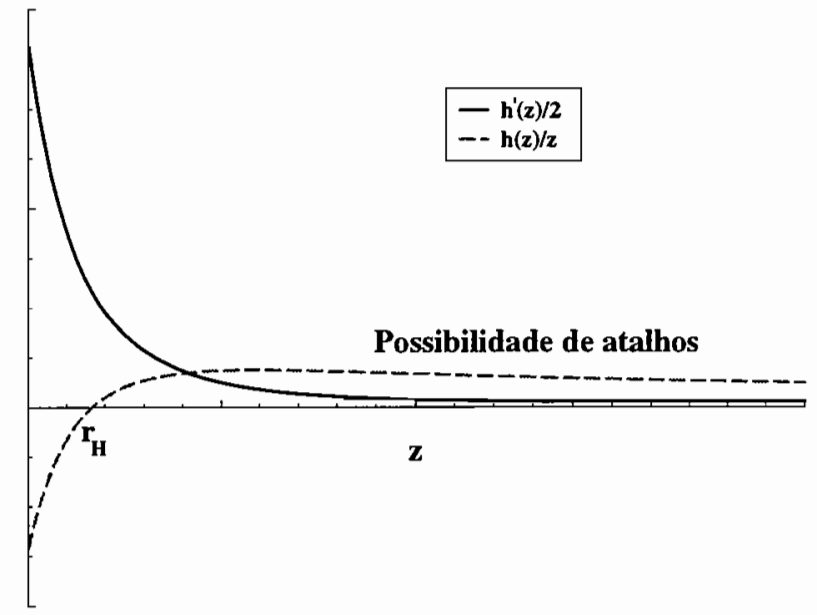

Figura 5.1: $h(z) / z$ e $h^{\prime}(z) / 2$ no bulk AdS-Schwarzschild em 6 dimensões. A região onde há possibilidade de atalhos corresponde à condição (5.9).

Levando em conta (5.6) e o fato que $\varepsilon^{2}$ deve ser positivo em (5.7) ${ }^{1}$

$$
-4(\omega+1) \varepsilon^{2} z_{0}^{2}<0,
$$

então

$$
\omega+1>0 .
$$

Agora vejamos as condições sob as quais um horizonte de eventos deve aparecer. Em geral, os horizontes acontecem nos zeros de $h(z)$, ou equivalentemente nos zeros de

$$
z^{5}+z^{3} l^{2}-l^{2} M
$$

Assim também, os zeros não nulos de $h^{\prime}(z)$ ocorrem quando

$$
2 z^{5}+3 l^{2} M=0 \text {. }
$$

Já que a derivada não tem zeros positivos com $M>0$, existe só um horizonte. Assim, conforme $h(z)$ vai para $-\infty$ na origem, as condições

$$
M>0
$$

e

$$
h\left(z_{0}\right)>0
$$

\footnotetext{
${ }^{1}$ Desde agora, chamaremos $\varepsilon^{2}=\kappa_{(6)}^{4} \rho^{2} / 64$ em 6 dimensões.
} 
são necessárias e, de fato, suficientes para se ter um horizonte e assegurar que a brana esteja depois dele.

A condição (5.14) é automaticamente satisfeita devido a (5.4).

Para cumprir com (5.13), vamos substituir (5.7) em (5.6) e teremos

$$
\left(\omega+\frac{3}{4}\right)+(\omega+1) \frac{z_{0}^{2}}{l^{2}}<0 .
$$

Se $\omega+1 \leq 0$, esta condição é sempre satisfeita, mas esta configuração não produz atalhos como gostaríamos. Porém, a condição é também satisfeita com $\omega+1>0$, se impusermos

e

$$
-1<\omega<-\frac{3}{4}
$$

$$
\frac{z_{0}^{2}}{l^{2}}<-\frac{\omega+3 / 4}{\omega+1}
$$

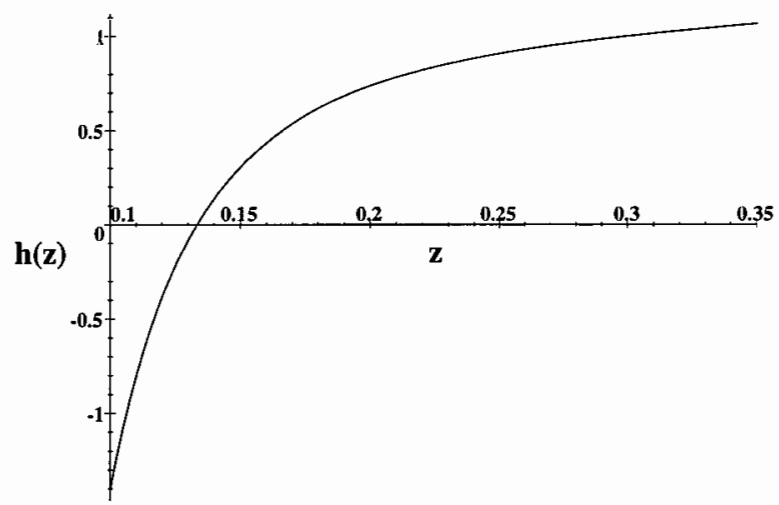

Figura 5.2: $h(z)$ no bulk AdS-Schwarzschild em 6 dimensões com a brana localizada em $z=1 / 3$. Note que a singularidade está protegida por um horizonte.

Se seguirmos (5.15) e (5.16), junto com o ajuste fino para a energia (5.7), teremos vários atalhos em bulks AdS-Schwarzschild com singularidade protegida. Nas Figs. 5.2 e 5.3, ilustramos um exemplo com $\omega=-4 / 5, z_{0}=1 / 3$, e $l=1$. Note que na Fig. 5.2 , o horizonte aparece antes da brana.

Já que este caso é equivalente a considerar o problema em 5 dimensões com $h(z)$, $M$ e $\rho$, dados em [24], resultados análogos são obtidos. Neste caso, o ajuste fino na 


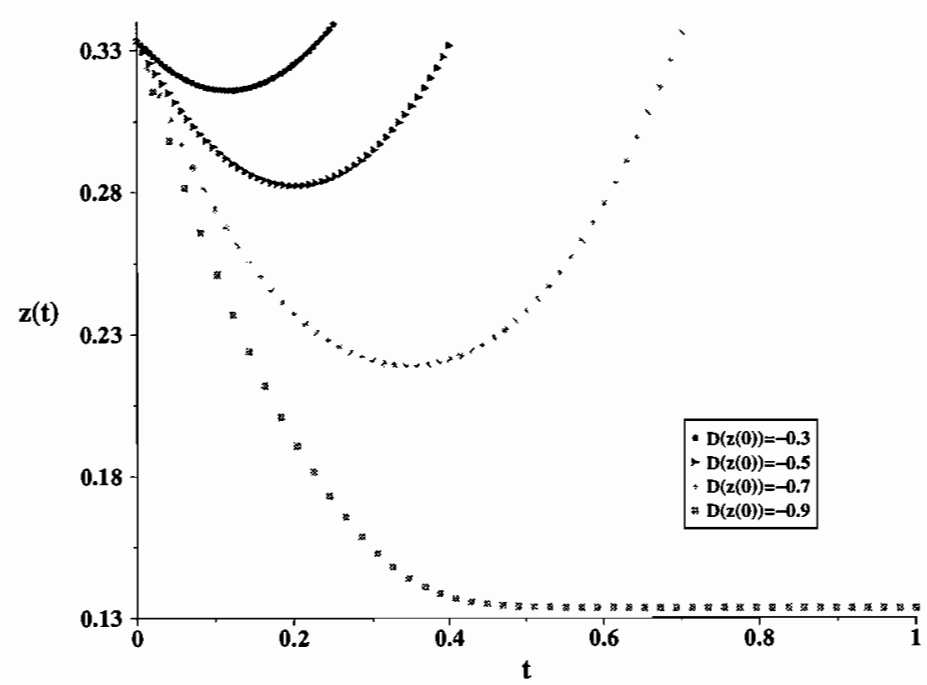

Figura 5.3: Atalhos para diversas velocidades iniciais no bulk AdS-Schwarzschild em 6 dimensões. Note que existe uma velocidade limiar para a qual o gráviton não pode retornar à brana e cai no horizonte de eventos.

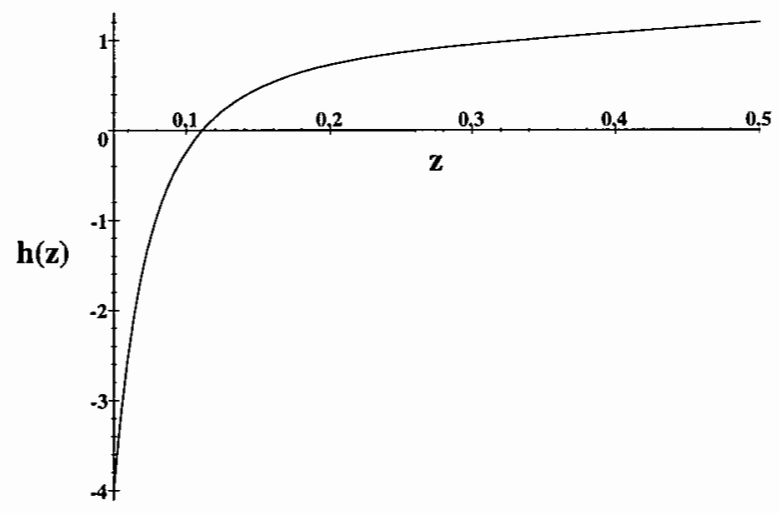

Figura 5.4: $h(z)$ no bulk AdS-Schwarzschild em 5 dimensões com a brana localizada em $z=1 / 2$. Note que a singularidade está protegida por um horizonte. 


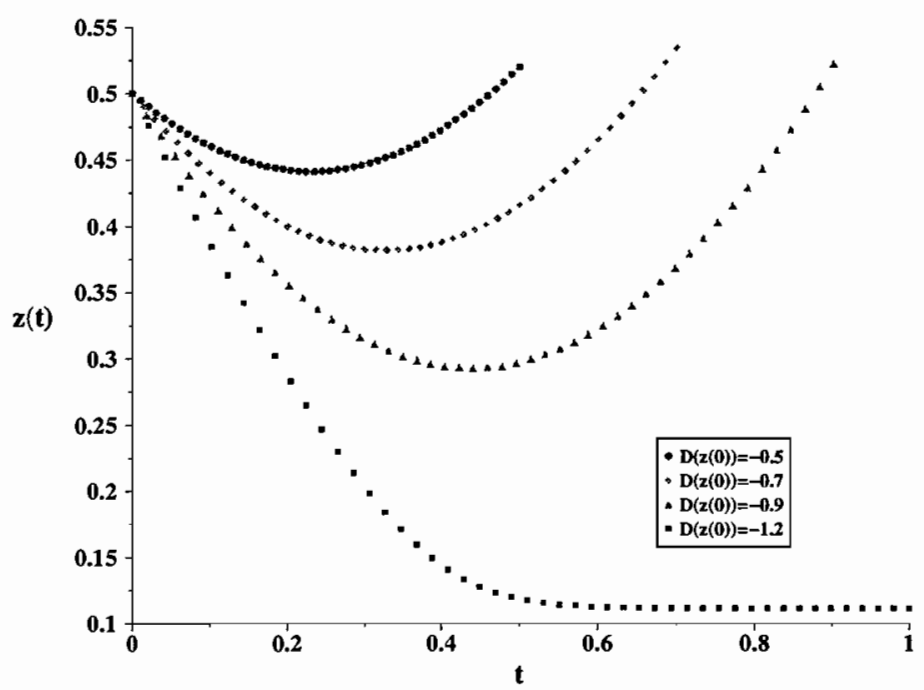

Figura 5.5: Atalhos para várias velocidades iniciais no bulk AdS-Schwarzschild em 5 dimensões. Como no caso 6-dimensional, existe uma velocidade inicial limiar para a qual o gráviton não pode voltar à brana e cai no horizonte de eventos.

eaergia é dado por ${ }^{2}$

$$
\varepsilon_{(5)}^{2}=-\frac{1}{3 \omega+1}\left(\frac{1}{z_{0}^{2}}+\frac{2}{l^{2}}\right)
$$

e $\omega$ está confinado a

$$
-1<\omega<-\frac{2}{3},
$$

enquanto que a posição da brana é dada por

$$
\frac{z_{0}^{2}}{l^{2}}<-\frac{\omega+2 / 3}{\omega+1}
$$

Um exemplo é mostrado nas Figs. 5.4 e 5.5 para $\omega=-3 / 4, z_{0}=1 / 2$, e $l=1$.

\subsection{O Bulk AdS-Reissner-Nordström}

O cenário AdS-Reissner-Nordström é descrito pela métrica

$$
d s^{2}=-h(z) d t^{2}+\frac{z^{2}}{l^{2}} d \Sigma_{k}^{2}+h^{-1}(z) d z^{2},
$$

\footnotetext{
${ }^{2}$ Neste caso, $\varepsilon_{(5)}^{2}=\kappa_{(5)}^{4} \rho^{2} / 36$.
} 
onde novamente

$$
d \Sigma_{k}^{2}=\frac{d r^{2}}{1-k r^{2}}+r^{2} d \Omega_{(2)}^{2}+\left(1-k r^{2}\right) d y^{2}
$$

e, neste caso,

$$
h(z)=k+\frac{z^{2}}{l^{2}}-\frac{M}{z^{3}}+\frac{Q^{2}}{z^{6}} .
$$

Das condições de Darmois-Israel (4.21), teremos para a massa e a carga do buraco negro,

$$
\begin{aligned}
\frac{M}{z_{0}^{5}} & =\frac{2 k}{z_{0}^{2}}+\frac{8}{3 l^{2}}+\frac{\kappa_{(6)}^{4}}{24} \rho^{2} \omega \\
\frac{Q^{2}}{z_{0}^{8}} & =\frac{k}{z_{0}^{2}}+\frac{5}{3 l^{2}}+\frac{8 \omega+3}{3} \frac{\kappa_{(6)}^{4} \rho^{2}}{64}
\end{aligned}
$$

Neste ponto, é conveniente estudar cuidadosamente a possibilidade de existência de atalhos para cada valor de $k$. A situação para $k=1$ pode ser visualizada na Fig.5.6.

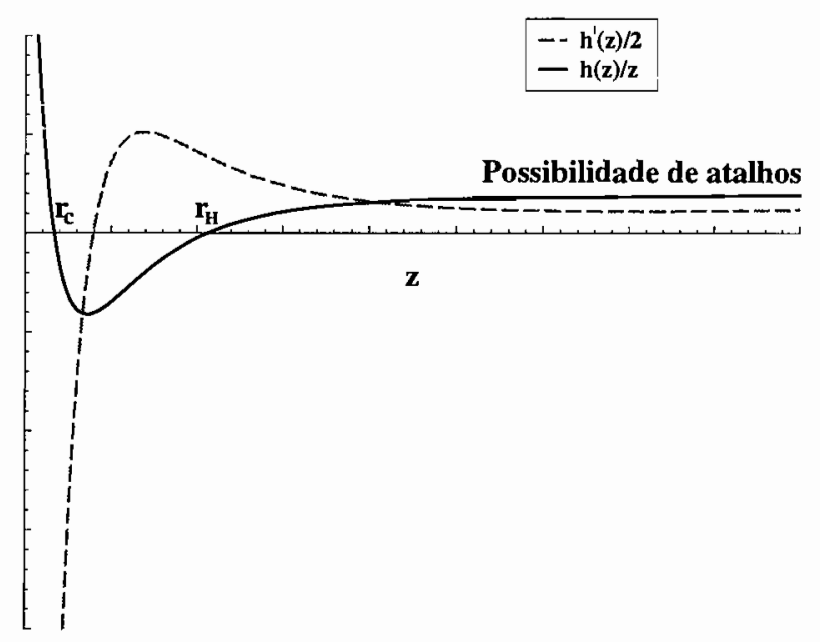

Figura 5.6: $h(z) / z$ e $h^{\prime}(z) / 2$ no bulk AdS-Reissner-Nordström em 6 dimensões. A região onde há possibilidade de atalhos corresponde à condição (5.9). 


\subsubsection{O Caso $k=0$ e $k=-1$}

Como foi achado no caso Schwarzschild, (5.9) determina a existência de atalhos. Usando (4.17), vemos que (5.9) tem um zero em $z=z_{f} \neq 0$ quando

$$
\frac{5}{2} M z_{f}^{3}-4 Q^{2}-k z_{f}^{6}=0
$$

Se $k=0$, temos uma raiz real em

$$
z_{f}^{3}=\frac{8 Q}{5 M}
$$

Se $k=1$, temos duas raízes em

$$
z_{f}^{3}=\frac{5}{4} M \pm \frac{1}{4} \sqrt{25 M^{2}-64 Q^{2}}
$$

Finalmente, se $k=-1$, temos

$$
z_{f}^{3}=-\frac{5}{4} M \pm \frac{1}{4} \sqrt{25 M^{2}+64 Q^{2}}
$$

INote que $F(z)$ tem no máximo um zero real e positivo se $k=0,-1$ e no máximo dois zeros positivos se $k=1$.

Analisando $h(z)$ e suas derivadas, vemos que $h(z)$ tende a $+\infty$ na singularidacie e no infinito, enquanto que $h^{\prime}(z)$ tende a $-\infty$ na singularidade e a $+\infty$ no infinito.

Os horizontes ocorrem nos zeros de $h(z)$, ou equivalentemente, nos zeros de

$$
z^{8}+l^{2} k z^{6}-l^{2} M z^{3}+l^{2} Q^{2}=0 .
$$

De outro lado, os zeros não nulos de $h^{\prime}(z)$ ocorrem quando

$$
2 z^{8}+3 l^{2} M z^{3}-6 l^{2} Q^{2}=0 .
$$

Este polinômio cresce no infinito e é negativo na origem. Sua derivada tem raízes não nulas quando

$$
16 z^{5}+9 l^{2} M=0 \text {. }
$$

Para $M>0$, esta equação nunca é satisfeita. Assim, como a derivada de (5.29) não se anula e é positiva fora da origem, o polinômio (5.29) cresce monotonicamente e tem só uma raiz. Os zeros deste polinômio são todos zeros não nulos de $h^{\prime}(z)$. Portanto, concluímos que para massa positiva, só existe um zero para $h^{\prime}(z)$, e portanto, no máximo dois horizontes para $h(z)$.

Quando existe um horizonte, $h^{\prime}(z)$ é negativo antes dele e positivo depois, cruzando $h(z)$ no horizonte mesmo. Se existem dois horizontes, $h^{\prime}(z)$ se anula em um ponto 
entre o horizonte de Cauchy e o horizonte de eventos, sendo negativo antes deste ponto e positivo depois, enquanto que $h(z)$ é positivo em todos os pontos exceto entre ambos os horizontes. Levando em conta o sinal e os zeros destas funções, $h^{\prime}(z)$ cruza $h(z)$ entre o horizonte de Cauchy e o ponto em que $h^{\prime}(z)$ se anula.

Já que $h^{\prime}(z) / 2$ tem o mesmo sinal que $h^{\prime}(z)$ e anula-se no mesmo ponto, e da mesma forma $h(z) / z$ tem o mesmo sinal de $h(z)$ e anula-se nos mesmos pontos, concluímos que, existindo horizontes, $F(z)$ necessariamente se anula em algum ponto $z=z_{c}$, tal que $0<z_{c}<z_{h}$. Porém, como foi dito antes, para $k=0$ ou $k=-1$, existe só uma raiz positiva de $F(z)$. Como $F(z)<0$ para $z<z_{c}$, então $F(z)>0$ para $z>z_{c}$. Assim, já que $z_{c} \leq z_{h}, F(z)>0$ para $z>z_{h}$, contrário ao que era requerido em (5.9). Isto implica que não existem atalhos com $k=0$ ou $k=-1$, quando existem horizontes.

Em 5 dimensões a prova é muito similar e chegamos à mesma conclusão.

\subsubsection{O Caso $k=1$}

Como vimos na seção anterior, $F(z)$ tem duas raízes reais, positivas e distintas para $k=1$,

$$
\begin{aligned}
& r_{1}^{3}=\frac{5}{4} M-\frac{1}{4} \sqrt{25 M^{2}-64} \overline{Q^{2}}, \\
& r_{2}^{3}=\frac{5}{4} M+\frac{1}{4} \sqrt{25 M^{2}-64 Q^{2}} .
\end{aligned}
$$

Esta é a única situação onde os atalhos podem coexistir com uma singularidade protegida. De fato, esta situação necessariamente requer que a segunda raiz de $F(z)$ esteja em algum ponto antes da posição da brana $z_{0}$. Isto também implica $F\left(z_{0}\right)<0$.

Além disso, devemos ter $Q^{2}$ e $M$ positivos.

Dado o fato que temos horizontes, se a brana não está entre eles ou na posição de algum horizonte, então $h\left(z_{0}\right)>0$. Ainda mais, para garantir que a brana esteja localizada depois do horizonte de eventos, também precisamos $h^{\prime}\left(z_{0}\right)>0$.

Da discussão na seção prévia, teremos um ou dois horizontes se e somente se $h\left(r_{1}\right) \leq 0$.

Resumindo, os atalhos em bulks com singularidades protegidas podem acontecer só se $k=1$ e somente se as condições seguintes são fornecidas:

1. $h\left(z_{0}\right)>0$ e $h^{\prime}\left(z_{0}\right)>0$, para ter ambos horizontes antes da brana.

2. $F\left(z_{0}\right)<0$ e $r_{2}<z_{0}$, para ter atalhos com singularidade protegida.

3. $Q^{2}>0$ e $M>0$, que garante a positividade da massa e da carga do buraco negro. 
4. $h\left(r_{1}\right) \leq 0$, para ter horizontes.

Vamos analisar cada condição e impor certas restrições em $\omega, \rho^{2}$, e $z_{0}$.

\section{Existência de Ambos Horizontes Antes da Brana}

Estas condições são as mais simples de analisar, já que elas restringem $\omega$ diretamente das condições de Darmois-Israel (4.21) junto com (4.22)

$$
\begin{aligned}
\frac{h\left(z_{0}\right)}{z_{0}^{2}} & =\varepsilon^{2}, \\
\frac{h^{\prime}\left(z_{0}\right)}{2 z_{0}} & =-(4 \omega+3) \varepsilon^{2} .
\end{aligned}
$$

A condição (5.33) é automaticamente satisfeita desde que $\varepsilon^{2}>0$.

Da condição (5.34)

$$
-(4 \omega+3) \varepsilon^{2}>0
$$

temos uma primeira restrição

$$
\omega<-3 / 4
$$

\section{Existência de Atalhos com Singularidade Protegida}

Da definição de $F(z),(5.9)$, e usando (5.33) e (5.34), vemos que

$$
0>F\left(z_{0}\right)=-4(\omega+1) \varepsilon^{2} z_{0} .
$$

Assim, achamos uma outra condição sobre $\omega$

$$
\omega+1>0
$$

Além disso, de $r_{2}<z_{0}$

$$
\frac{5 M}{4}-z_{0}^{3}<-\frac{1}{4} \sqrt{25 M^{2}-64 Q^{2}} .
$$

Esta equação será satisfeita se ${ }^{3}$

$$
\frac{5 M}{4}-z_{0}^{3}<0
$$

${ }^{3}$ Assumimos que $25 M^{2}-64 Q^{2}>0$. Retornaremos a esta condição quando discutamos a existência de horizontes, onde imporemos uma restrição mais forte, $M^{2}-4 Q^{2}>0$. 
ou usando (5.23)

$$
\frac{3}{2} z_{0}^{3}+\frac{10}{3} \frac{z_{0}^{5}}{l^{2}}+\frac{10}{3} z_{0}^{5} \omega \varepsilon^{2}<0
$$

e como $\omega<-3 / 4$

$$
z_{0}^{2} \varepsilon^{2}>\frac{1}{\omega}\left(-\frac{9}{20}-\frac{z_{0}^{2}}{l^{2}}\right)
$$

\section{Positividade da Massa e da Carga do Buraco Negro}

Já que requeremos a positividade da massa do buraco negro, de (5.23) temos

$$
\frac{M}{z_{0}^{3}}>0 \Rightarrow \frac{z_{0}^{2}}{l^{2}}+\omega \varepsilon^{2} z_{0}^{2}>-\frac{3}{4},
$$

assim,

$$
z_{0}^{2} \varepsilon^{2}<\frac{1}{\omega}\left(-\frac{3}{4}-\frac{z_{0}^{2}}{l^{2}}\right) .
$$

Já que $3 / 4>9 / 20$, esta condição é certamente compatível com (5.42).

De outro lado, a positividade do quadrado da carga do buraco negro requer

$$
\frac{Q^{2}}{z_{0}^{6}}>0 \Rightarrow 1+\frac{5 z_{0}^{2}}{3 l^{2}}+\left(\frac{8}{3} \omega+1\right) z_{0}^{2} \varepsilon^{2}>0
$$

tal que

$$
z_{0}^{2} \varepsilon^{2}<\frac{1}{8 \omega+3}\left(-3-\frac{5 z_{0}^{2}}{l^{2}}\right) .
$$

Apesar de não ser trivial, esta equação é também compatível com (5.42). Isto requer

$$
\frac{1}{\omega}\left(-\frac{9}{20}-\frac{z_{0}^{2}}{l^{2}}\right)<\frac{1}{8 \omega+3}\left(-3-\frac{5 z_{0}^{2}}{l^{2}}\right),
$$

$\mathrm{ou}$

$$
-\frac{1}{5}\left(\omega+\frac{9}{4}\right)-\frac{z_{0}^{2}}{l^{2}}(\omega+1)<0,
$$

que é sempre verdadeiro para $-1<\omega<-3 / 4$.

\section{Existência de Horizontes}

Esta é a última e a mais complicada das nossas condições. Devemos ter $h\left(r_{1}\right) \leq 0$. Seja $x=r_{1}^{3}$,

$$
\frac{x^{8 / 3}}{l^{2}}+x^{2}-M x+Q^{2} \leq 0
$$


Não precisamos fazer um estudo completo desta equação. Para nossos propósitos será suficiente impor

$$
x^{2}-M x+Q^{2}<0
$$

Usando (5.31), isto implica

$$
M^{2}-4 Q^{2}>0
$$

Esta condição é necessária mas não suficiente para ter horizontes. Porém, esta restrição acrescentada às outras desenvolvidas nesta seção, serão suficientes para construir atalhos com horizontes como veremos. Note que esta condição é mais forte que aquela assumida anteriormente, $M>8 / 5 Q$.

Usando (5.23) em (5.51),

$$
\left(1-\varepsilon^{2} l^{2}\right)+\frac{16}{9} \frac{z_{0}^{2}}{l^{2}}\left(1+\omega \varepsilon^{2} l^{2}\right)^{2}>0
$$

Sabemos de (5.42) que $1-\varepsilon^{2} l^{2}$ deve ser negativo. Portanto, devemos analisar cuidadosamente (5.52). Podemos interpretar (5.52) como uma equação quadrática na energia

$$
\left(1+\frac{16}{9} \frac{z_{0}^{2}}{l^{2}}\right)+\left(\frac{32}{9} \omega z_{0}^{2}-l^{2}\right) \varepsilon^{2}+\left(\frac{16}{9} l^{2} z_{0}^{2} \omega^{2}\right) \varepsilon^{4}>0
$$

que implica

$$
z_{0}^{2} \varepsilon^{2}>\frac{1}{32}\left(-32 \omega z_{0}^{2}+9 l^{2}+3 \sqrt{-64 \omega z_{0}^{2} l^{2}+9 l^{4}-64 l^{2} z_{0}^{2} \omega^{2}}\right) /\left(\omega^{2} l^{2}\right)
$$

ou

$$
z_{0}^{2} \varepsilon^{2}<\frac{1}{32}\left(-32 \omega z_{0}^{2}+9 l^{2}-3 \sqrt{-64 \omega z_{0}^{2} l^{2}+9 l^{4}-64 l^{2} z_{0}^{2} \omega^{2}}\right) /\left(\omega^{2} l^{2}\right)
$$

Note que, já que $-1<\omega<-3 / 4$,

$$
-64 \omega z_{0}^{2} l^{2}-64 l^{2} z_{0}^{2} \omega^{2}=-64 \omega(\omega+1) z_{0}^{2} l^{2}>0
$$

e todas as raízes prévias são reais e positivas.

Resumindo, das considerações nas seções anteriores, devemos ter para $\omega$

$$
-1<\omega<-3 / 4
$$

Para a energia,

$$
\begin{aligned}
\frac{1}{\omega}\left(-\frac{9}{20}-\frac{z_{0}^{2}}{l^{2}}\right)<z_{0}^{2} \varepsilon^{2}<\frac{1}{\omega}\left(-\frac{3}{4}-\frac{z_{0}^{2}}{l^{2}}\right), & \text { ou } \\
\frac{1}{\omega}\left(-\frac{9}{20}-\frac{z_{0}^{2}}{l^{2}}\right)<z_{0}^{2} \varepsilon^{2}<\frac{1}{8 \omega+3}\left(-3-\frac{5 z_{0}^{2}}{l^{2}}\right), &
\end{aligned}
$$


dependendo qual condição for mais restritiva.

Além disso,

$$
\begin{aligned}
& z_{0}^{2} \varepsilon^{2}>\frac{1}{32}\left(-32 \omega z_{0}^{2}+9 l^{2}+3 \sqrt{-64 \omega z_{0}^{2} l^{2}+9 l^{4}-64 l^{2} z_{0}^{2} \omega^{2}}\right) /\left(\omega^{2} l^{2}\right) \\
& z_{0}^{2} \varepsilon^{2}<\frac{1}{32}\left(-32 \omega z_{0}^{2}+9 l^{2}-3 \sqrt{-64 \omega z_{0}^{2} l^{2}+9 l^{4}-64 l^{2} z_{0}^{2} \omega^{2}}\right) /\left(\omega^{2} l^{2}\right)
\end{aligned}
$$

Agora vamos analisar as situações em que todas estas condições sejam compatíveis.

Comecemos nossa análise com a equação (5.60). Para que esta seja compatível com (5.57) e (5.58), só precisamos

$$
\frac{1}{\omega}\left(-\frac{9}{20}-\frac{z_{0}^{2}}{l^{2}}\right)<\frac{1}{32}\left(-32 \omega z_{0}^{2}+9 l^{2}-3 \sqrt{-64 \omega z_{0}^{2} l^{2}+9 l^{4}-64 l^{2} z_{0}^{2} \omega^{2}}\right) /\left(\omega^{2} l^{2}\right),
$$

isto é,

$$
-\frac{9}{20} \omega-\frac{9}{32}+\frac{3}{32} \sqrt{-64 \omega \frac{z_{0}^{2}}{l^{2}}+9-64 \frac{z_{0}^{2}}{l^{2}} \omega^{2}}<0
$$

Já que $3 / 4<|\omega|<1$,

$$
-\frac{9}{20} \omega-\frac{9}{32}
$$

será semp pe positivo e assim, (5.62) nunca será satisfeita. Então, concluímos que (5.60) não é compatível nem com (5.57), nem com (5.58). Isto implica que $z_{0}^{2} \varepsilon^{2}$ deve satisfazer (5.59), junto com (5.57) ou (5.58).

Comparemos inicialmente (5.57) com (5.59). Devemos ter

$$
\frac{1}{\omega}\left(-\frac{3}{4}-\frac{z_{0}^{2}}{l^{2}}\right)>\frac{1}{32}\left(-32 \omega z_{0}^{2}+9 l^{2}+3 \sqrt{-64 \omega z_{0}^{2} l^{2}+9 l^{4}-64 l^{2} z_{0}^{2} \omega^{2}}\right) /\left(\omega^{2} l^{2}\right),
$$

isto é,

$$
-\frac{3}{4} \omega-\frac{9}{32}-\frac{3}{32} \sqrt{-64 \omega \frac{z_{0}^{2}}{l^{2}}+9-64 \frac{z_{0}^{2}}{l^{2}} \omega^{2}}>0 .
$$

Neste caso, desde que $\omega$ é negativo e $3 / 4<|\omega|<1$,

$$
-\frac{3}{4} \omega-\frac{9}{32}>0
$$

e (5.64) pode ser satisfeito se

$$
\left(-\frac{3}{4} \omega-\frac{9}{32}\right)^{2}>\frac{9}{1024}\left(-64 \omega \frac{z_{0}^{2}}{l^{2}}+9-64 \frac{z_{0}^{2}}{l^{2}} \omega^{2}\right)
$$

ou

$$
\frac{9}{16} \omega(\omega+1) \frac{z_{0}^{2}}{l^{2}}+\frac{9}{64}(3+4 \omega) \omega>0 .
$$


Já que $\omega+1>0$ e $3+4 \omega<0$, para $z_{0}$ positivo a desigualdade será somente satisfeita se

$$
\frac{z_{0}}{l}<\frac{1}{2} \sqrt{-\frac{3+4 \omega}{1+\omega}}
$$

Tal que (5.59) e (5.57) possam ser compatíveis.

Agora vamos analisar a compatibilidade entre (5.59) e (5.58). Devemos ter

$$
\frac{1}{8 \omega+3}\left(-3-\frac{5 z_{0}^{2}}{l^{2}}\right)+\frac{z_{0}^{2}}{\omega l^{2}}-\frac{9}{32 \omega^{2}}>\frac{3}{32 \omega^{2}} \sqrt{-64 \omega z_{0}^{2} l^{2}+9 l^{4}-64 l^{2} z_{0}^{2} \omega^{2}}
$$

ou usando que $8 \omega+3<0$, simplificando e elevando ao quadrado ambos lados, podemos escrever (5.68) como

$$
\frac{1}{16} \omega^{2}(3+4 \omega)^{2}+\omega^{2}(\omega+1)^{2}\left(\frac{z_{0}^{2}}{l^{2}}\right)^{2}+\frac{\omega^{2}}{2}(3+4 \omega)(\omega+1) \frac{z_{0}^{2}}{l^{2}}>0 .
$$

Este polinômio tem somente uma raiz para $z_{0}^{2} / l^{2}$

$$
\frac{z_{0}^{2}}{l^{2}}=-\frac{1}{4}\left(\frac{3+4 \omega}{1+\omega}\right)
$$

Já que o coeficiente de $z_{0}^{4} / l^{4}$ é positivo, a desigualdade é satisfeita com a mesma colıdição (5.67), assim, verificamos que (5.58) e (5.57) são compatíveis coln (5.59) sob as mesmas restrições.

Ainda mais, comparemos os limites superiores de (5.57) e (5.58). Suponhamos

$$
\frac{1}{\omega}\left(-\frac{3}{4}-\frac{z_{0}^{2}}{l^{2}}\right)>\frac{1}{8 \omega+3}\left(-3-\frac{5 z_{0}^{2}}{l^{2}}\right)
$$

que também pode ser escrito como

$$
-\frac{3}{4}(4 \omega+3)-\frac{3 z_{0}^{2}}{l^{2}}(\omega+1)>0 .
$$

Assim, (5.70) é satisfeito se e somente si

$$
\frac{z_{0}^{2}}{l^{2}}<-\frac{1}{4}\left(\frac{3+4 \omega}{1+\omega}\right)
$$

que é justamente a mesma desigualdade (5.67), que $z_{0}$ deve satisfazer. Portanto, entre (5.57) e (5.58), é suficiente levar em conta a última. Porém, de (5.69) note que (5.58) seria também compatível com (5.59) se

$$
\frac{z_{0}^{2}}{l^{2}}>-\frac{1}{4}\left(\frac{3+4 \omega}{1+\omega}\right)
$$


e (5.70) seria satisfeita com uma mudança de sinal, implicando que deveríamos considerar (5.57) no lugar de (5.58), mas, como foi dito antes, a compatibilidade de (5.57) e (5.59) requer

$$
\frac{z_{0}^{2}}{l^{2}}<-\frac{1}{4}\left(\frac{3+4 \omega}{1+\omega}\right)
$$

que contradiz nossa hipótese. Portanto, a única configuração possível é (5.72).

Finalmente, comparemos os limites inferiores de (5.58) e (5.59). Suponhamos

$$
\frac{1}{\omega}\left(-\frac{9}{20}-\frac{z_{0}^{2}}{l^{2}}\right)<\frac{1}{32 \omega^{2} l^{2}}\left(-32 \omega z_{0}^{2}+9 l^{2}+3 \sqrt{-64 \omega z_{0}^{2} l^{2}+9 l^{4}-64 l^{2} z_{0}^{2} \omega^{2}}\right),
$$

ou

$$
-\frac{9}{20} \omega-\frac{9}{32}<\frac{3}{32} \sqrt{-64 \omega \frac{z_{0}^{2}}{l^{2}}+9-64 \frac{z_{0}^{2}}{l^{2}} \omega^{2}} .
$$

Elevando ao quadrado e simplificando, obtemos

$$
\frac{z_{0}^{2}}{l^{2}}>-\frac{9}{20(\omega+1)}\left(\frac{4}{5} \omega+1\right) \text {. }
$$

Para $-1<\omega<-3 / 4$, esta desigualdade é sempre satisfeita já que o lado direito é negauivo. Portanto, concluímos que (5.73) é valida e, entre os limites inferiores para a energia em (5.58) e (5.59), somente precisamos escolher o último.

Em resumo, por considerações puramente analíticas concluímos que os atalhos em bulks sem singularidades nuas possuindo uma brana estática imersa, podem somente aparecer se $k=1$ e se as condições seguintes são satisfeitas:

1. Devemos escolher $\omega$ tal que $-1<\omega<-3 / 4$;

2. Dado $\omega$, a brana deve estar localizada numa posição tal que

$$
\frac{z_{0}}{l}<\frac{1}{2} \sqrt{-\frac{3+4 \omega}{1+\omega}}
$$

que é a mesma condição que no caso AdS-Schwarzschild (5.16);

3. Dado (5.74), a energia $\varepsilon$ deve satisfazer

$$
\frac{1}{32 \omega^{2}}\left(-\frac{32 \omega z_{0}^{2}}{l^{2}}+9+3 \sqrt{-64 \omega \frac{z_{0}^{2}}{l^{2}}+9-64 \frac{z_{0}^{2}}{l^{2}} \omega^{2}}\right)<z_{0}^{2} \varepsilon^{2}<\frac{1}{8 \omega+3}\left(-3-\frac{5 z_{0}^{2}}{l^{2}}\right) .
$$

Desta forma, se faz simples achar atalhos em bulks com singularidades protegidas. 


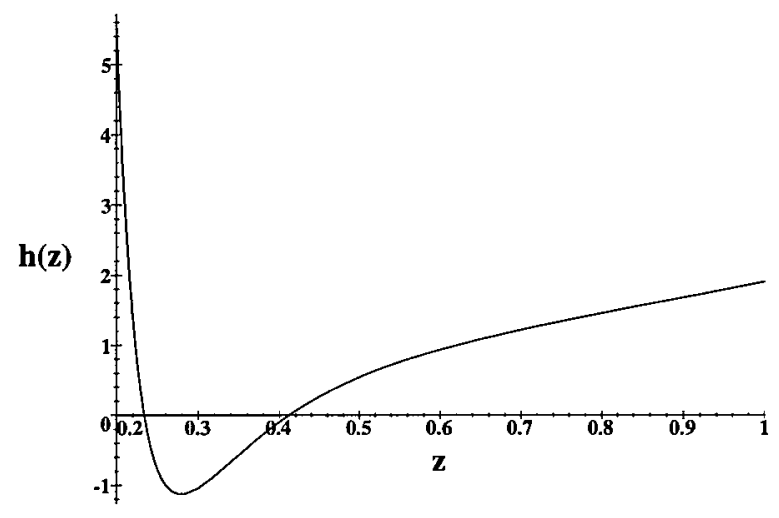

Figura 5.7: $h(z)$ no bulk AdS-Reissner-Nordström em 6 dimensões com a brana localizada em $z=1$. Note que a singularidade está protegida por dois horizontes.

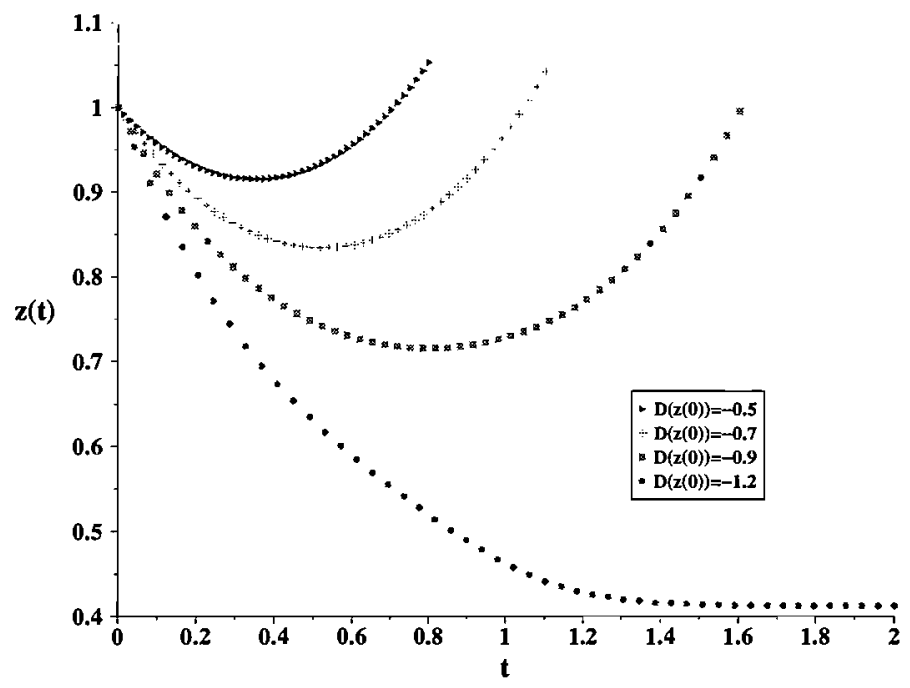

Figura 5.8: Atalhos para várias velocidades iniciais no bulk AdS-Reissner-Nordström em 6 dimensões. Note que existe uma velocidade inicial limiar para a qual o gráviton não pode voltar à brana e cai no horizonte de eventos. 


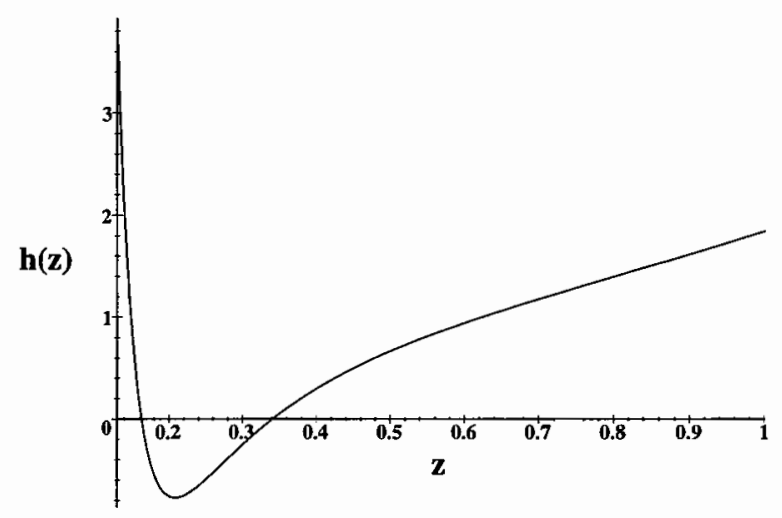

Figura 5.9: $h(z)$ no bulk AdS-Reissner-Nordström em 5 dimensões com a brana localizada em $z=1$. Vemos que a singularidade está protegida por 2 horizontes.

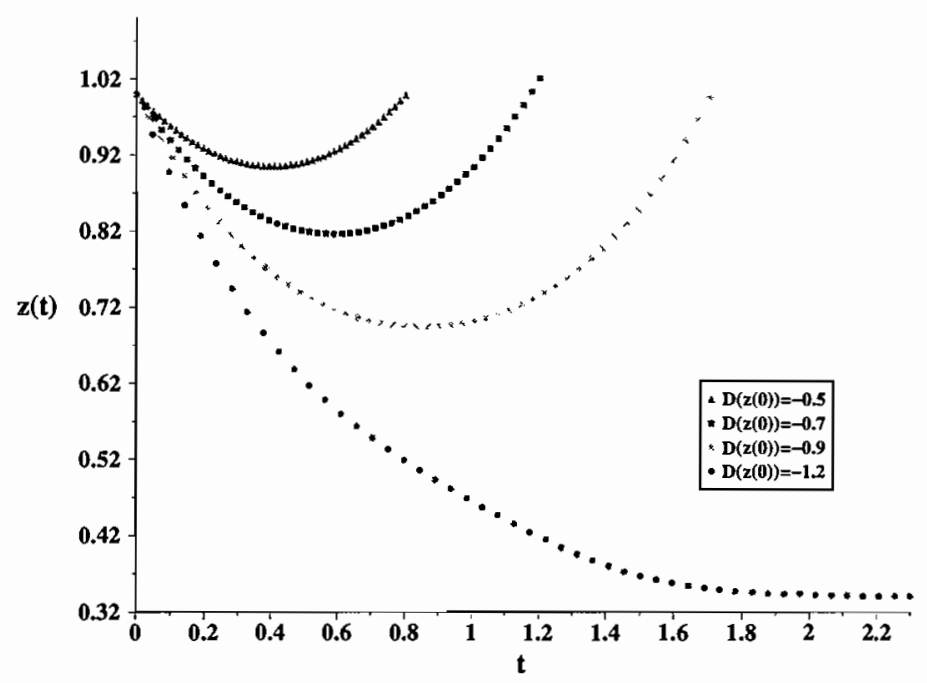

Figura 5.10: Atalhos para diferentes velocidades iniciais no bulk AdS-ReissnerNordström em 5 dimensões. Vemos que existe uma velocidade inicial limiar para a qual o gráviton não pode retornar à brana e cai no horizonte de eventos. 
Como um exemplo, vamos escolher $\omega=-9 / 10$. De (5.74), devemos ter

$$
\frac{z_{0}}{l}<\frac{\sqrt{6}}{2}
$$

então escolhemos $l=1$ e $z_{0}=1$.

De (5.75) temos

$$
\frac{35}{24}+\frac{5}{72} \sqrt{41}<\varepsilon^{2}<\frac{40}{21},
$$

assim escolhemos $\varepsilon=\sqrt{238 / 125}$.

Na Fig. (5.7) plotamos $h(z)$ com estas condições. Note que a singularidade está protegida por um horizonte de eventos e a brana está em $z=z_{0}=1$.

Na Fig. (5.8) plotamos as trajetórias do gráviton obtidas de (4.30) sob as condições prévias para uma variedade de velocidades iniciais (veja no Apêndice B um exemplo do programa em MAPLE que gerou os gráficos), mostrando que de fato os atalhos aparecem quando escolhemos os parâmetros seguindo o análise mostrado nesta seção.

A análise em 5 dimensões pode ser feito analogamente a aquele em 6 dimensões com

$$
h(z)=1+\frac{z^{2}}{z^{2}}-\frac{M}{z^{2}}+\frac{Q^{2}}{z^{4}}
$$

$\mathrm{e}$

$$
\begin{aligned}
& \frac{M}{z_{0}^{4}}=\frac{2}{z_{0}^{2}}+\frac{3}{l^{2}}+3 \omega \varepsilon_{(5)}^{2} \\
& \frac{Q^{2}}{z_{0}^{6}}=\frac{1}{z_{0}^{2}}+\frac{2}{l^{2}}+3 \omega \varepsilon_{(5)}^{2}+\varepsilon_{(5)}^{2}
\end{aligned}
$$

chegando às restrições seguintes:

1. Devemos escolher $\omega$ tal que $-1<\omega<-2 / 3$;

2. Dado $\omega$, a brana deve estar localizada numa posição tal que

$$
\frac{z_{0}}{l}<\sqrt{-\frac{\omega+2 / 3}{1+\omega}}
$$

3. Dado (5.79), a energia $\varepsilon_{(5)}^{2}=\kappa_{(5)}^{4} \rho^{2} / 36$ deve satisfazer

$$
\frac{1}{9 \omega^{2}}\left(2-9 \frac{z_{0}^{2}}{l^{2}} \omega+2 \sqrt{1-9 \frac{z_{0}^{2}}{l^{2}} \omega(\omega+1)}\right)<z_{0}^{2} \varepsilon_{(5)}^{2}<\frac{1}{3 \omega+1}\left(-1-\frac{2 z_{0}^{2}}{l^{2}}\right)
$$


Como um exemplo, escolhemos $\omega=-7 / 8$. De (5.79), devemos ter

$$
\frac{z_{0}}{l}<\frac{\sqrt{15}}{3},
$$

então escolhemos $l=1$ e $z_{0}=1$.

De (5.80) a energia deve satisfazer a

$$
\frac{632}{441}+\frac{16}{441} \sqrt{127}<\varepsilon_{(5)}^{2}<\frac{24}{13},
$$

então escolhemos $\varepsilon_{(5)}=\sqrt{461 / 250}$.

Na Fig. (5.9) podemos ver $h(z)$ de acordo com as condições prévias. Como no caso 6-dimensional, a singularidade está protegida por um horizonte de eventos e a brana está em $z=z_{0}=1$.

Na Fig. (5.10) mostramos várias trajetórias do gráviton obtidas sob as condições prévias para diferentes velocidades iniciais, mostrando que os atalhos aparecem quando escolhemos os parâmetros seguindo o análise mostrado nesta seção analogamente ao caso em 6 dimensões.

\subsection{Discussão dos Resultados}

Neste capítulo mostramos que a trajetória mais curta para o gráviton está governada por somente uma equação envolvendo a coordenada "radial" extra. Também vimos que a simetria na coordenada "angular" extra tem nos permitido considerar seções espaciais curvas.

Como foi dito nas seções 4 e 5.1, os casos $k=0$ e $k=-1$ não apresentam atalhos, levando à conclusão que $k=1$ é uma condição necessária para a existência de atalhos, o que concorda perfeitamente com a observação de Chung e Freese [31].

Desta maneira, os bulks AdS-Schwarzschild e AdS-Reissner-Nordström abrem a possibilidade de se ter atalhos sempre que a seção espacial tenha curvatura positiva e um conjunto de fortes restrições na tensão intrínseca da brana seja satisfeito. Da mesma forma, sua localização no bulk deve ser respeitada. Devemos também notar que a energia já sofre um ajuste fino por parte das condições de Darmois-Israel no caso do bulk AdS-Schwarzschild.

Portanto, é interessante notar que apesar do fato da carga contribuir a ter um $F\left(z_{0}\right)$ negativo e assim facilitar a existência de atalhos, existem condições ainda mais restritivas para a energia, que vêm de $Q^{2}>0$ e da equação de horizontes (5.49), que não aparecem no caso não carregado. Assim, os resultados favorecem a existência de atalhos em bulks com singularidades protegidas com as mesmas condições para $\omega$ e $z_{0}$ 
que no caso AdS-Schwarzschild, e também impõem o que é praticamente um ajuste fino na energia. Desta forma, ambos casos parecem ser equivalentes para o estudo de atalhos em universos estáticos com singularidades protegidas. Devemos notar que as restrições para obter estes atalhos fazem perder a principal vantagem que devíamos ter no estudo do caso AdS-Reissner-Nordström, i.e., a ausência de ajustes finos para a tensão intrínseca.

Apesar da existência de ajustes finos, o fato é que os atalhos aparecem e as conseqüências são muitas. Como foi mencionado em [29, 30], a existência de atalhos pode parcialmente resolver o problema do horizonte. Devemos notar que nosso conjunto de condições para obter atalhos em bulks AdS-Schwarzschild e AdS-Reissner-Nordström com singularidades protegidas impõe uma restrição no tamanho do universo, ou seja $z_{0} \sim l$, que corresponde a um universo primogêneo. Assim, os resultados aqui mostrados podem contribuir à solução deste problema importante.

Podemos também falar de conseqüências experimentais. Em particular, ondas gravitacionais avançadas com respeito aos fótons poderiam ser achadas nas antenas gravitacionais propostas e em operação, no caso de encontrarmos um modelo para o universo com um tamanho físico. 


\section{Capítulo 6}

\section{Atalhos em Paredes de Domínio e o Problema do Horizonte}

Neste capítulo, vamos considerar os modelos de Chamblin e Reall discutidos na seção 4.3. Assim, o cenário vai estar descrito por uma membrana dinâmica em um espaçotempo contendo matéria escalar descrita por paredes de domínio. Usando as soluções das equações de Einstein e as condições de Darmois-Israel, investigaremos a vossibilidade de ter atalhos para grávitons que deixam a parede e retornam subseqüent,emente. Como veremos, em comparação com os fótons que seguem uma geodésica dentro da brana, verificamos que os atalhos existem e aparecem sob condições bem leves. Para alguns universos eles são pequenos, mas há casos onde os atalhos são efetivos. Nestes casos, esperamos que joguem um papel significativo na solução do problema do horizonte, como discutimos no final do capítulo.

\subsection{O Cenário e a Equação de Atalhos}

Como vimos no capítulo 4, a métrica que descreve o cenário de Chamblin e Reall está dada por

$$
d s^{2}=-U(r) d t^{2}+U(r)^{-1} d r^{2}+R(r)^{2} d \Omega_{k}^{2}
$$

onde $d \Omega_{k}^{2}$ é o elemento de linha em um espaço $D$-2-dimensional de curvatura constante dependendo do parâmetro $k$. Impomos também uma simetria $Z_{2}$ a esta métrica.

A equação de movimento para a parede de domínio, obtida da componente $(i j)$ das condições de Darmois-Israel (4.45), pode ser escrita em termos do tempo próprio da brana $\tau$, como em (4.50)

$$
\frac{1}{2}\left(\frac{d R}{d \tau}\right)^{2}+F(R)=0
$$


A métrica induzida na parede de domínio é Friedmann-Robertson-Walker e (4.50) descreve a evolução do fator de escala $R(\tau)$. Esta equação é a mesma que aquela para uma partícula de massa unidade e energia nula, rolando em um potencial $F(R)$, dado por

$$
F(R)=\frac{1}{2} U R^{\prime 2}-\frac{1}{8(D-2)^{2}} \hat{V}^{2} R^{2} .
$$

Note que a solução existe somente quando $F(R) \leq 0$.

Da métrica induzida na parede de domínio,

$$
d s_{p d}^{2}=-d \tau^{2}+R(\tau)^{2} d \Omega_{k}^{2}
$$

achamos a relação entre o parâmetro de tempo na brana $(\tau)$ e aquele no bulk $(t)$,

$$
d t=\frac{\sqrt{U+\left(\frac{d r}{d \tau}\right)^{2}}}{U} d \tau
$$

tal que

$$
\dot{r} \equiv \frac{d r}{d t}=\frac{d r}{d \tau} \frac{d \tau}{d t}=\frac{d r}{d \tau} \frac{U}{\sqrt{U+\left(\frac{d r}{d \tau}\right)^{2}}},
$$

onde $d r / d \tau=(d R / d \tau)(d R / d r)^{-1}$ pode ser obtido com a ajuda de (4.50). A Eq. (6.6) descreve o movimento de uma parede de domínio no fundo estáticc, como visto por um observador no b'ılk.

Consicieremos dois pontos na brana. Em geral, existe mais de uma geodésica nula conectando-os no espaço-tempo D-dimensional. As trajetórias dos fótons devem estar na brana e aquelas dos grávitons podem estar fora. Consideremos o caminho mais curto para ambos, fótons e grávitons. Para os últimos, a equação geodésica é (4.30) [34], já que a métrica do bulk é estática:

$$
\ddot{r}_{g}+\left(\frac{1}{r_{g}}-\frac{3}{2} \frac{U^{\prime}}{U}\right) \dot{r}_{g}^{2}+\frac{1}{2} U U^{\prime}-\frac{U^{2}}{r_{g}}=0 .
$$

Note que o ponto indica derivada com respeito ao tempo do bulk $t$.

As soluções de (6.6) e (6.7) em termos do tempo próprio do bulk $t$ foram obtidas usando um programa em MAPLE [43]. Agora discutiremos a possibilidade de atalhos nos casos das variadas soluções descrevendo diferentes universos definidos pela solução da equação de movimento da parede de domínio.

\subsection{Soluções da Equação Geodésica}

\subsubsection{Soluções Tipo-I}

Como foi discutido no capítulo 4, as soluções tipo-I são definidas como aquelas que tem $\alpha=\beta=0$. Conseqüentemente, os potenciais tornam-se constantes cosmológicas. 
A solução tem também dílaton constante $\phi=\phi_{0}$. Um reescalonamento simples na métrica nos leva a

$$
d s^{2}=-U(R) d t^{2}+U(R)^{-1} d R^{2}+R^{2} d \Omega_{k}^{2}
$$

com

$$
U(R)=k-2 M R^{-(D-3)}-\frac{2 V_{0}}{(D-1)(D-2)} R^{2},
$$

que corresponde a uma solução de buraco negro topológico em $D$ dimensões com uma constante cosmológica.

Como é discutido em [56], se a parede de domínio tem densidade de energia positiva $\left(\hat{V}_{0}>0\right)$, a parte relevante do bulk é $R<R(\tau)$, que é a região contendo a singularidade. Pelo contrário, se a parece de domínio tem densidade de energia negativa $\left(\hat{V}_{0}<0\right)$, a parte relevante é $R>R(\tau)$, que não é singular a menos que a parede alcance $R=0$.
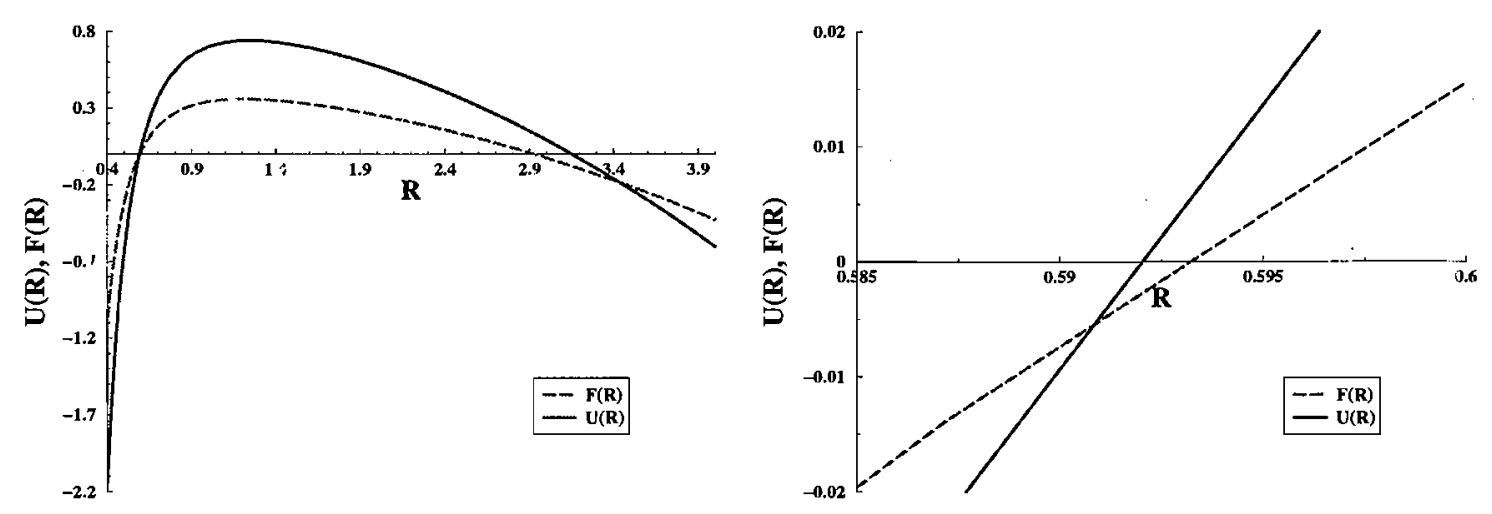

(a)

(b)

Figura 6.1: (a) $U(R)$ e $F(R)$ para as soluções tipo-I com $M=1 / 10, V_{0}=1$, e $\hat{V}_{0}= \pm 1$, (b) Aproximação da região do horizonte de eventos.

O potencial $F(R)$ que governa a evolução do fator de escala é

$$
F(R)=\frac{k}{2}-M R^{-(D-3)}-\hat{\Lambda} R^{2}
$$

onde a constante cosmológica efetiva na parede de domínio é dada por

$$
\hat{\Lambda}=\frac{1}{D-2}\left[\frac{V_{0}}{D-1}+\frac{\hat{V}_{0}^{2}}{8(D-2)}\right] \text {. }
$$

Analisaremos cada um dos 4 casos apresentados em [56] (para um exemplo em MAPLE, veja o apêndice C). Como dizemos anteriormente, a equação de movimento 


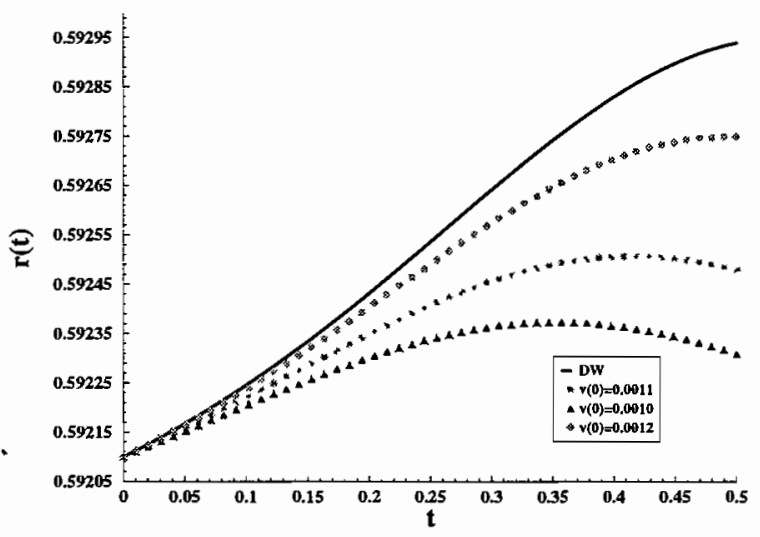

(a)

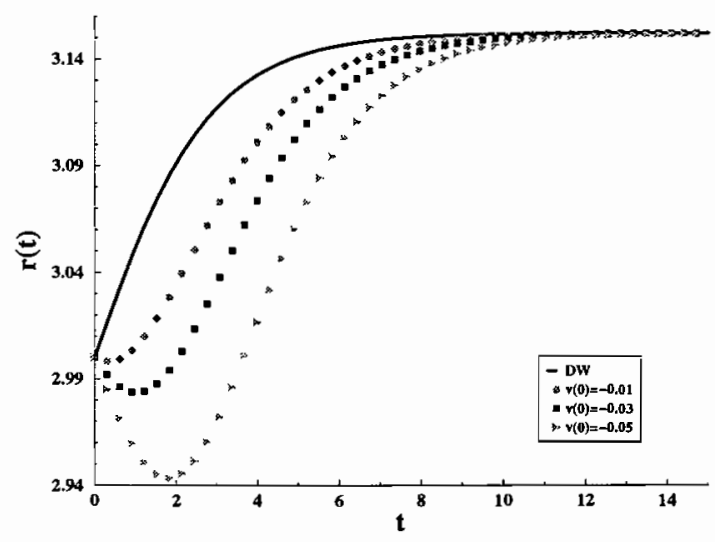

(b)

Figura 6.2: Movimento da parede de domínio e geodésicas para as soluções tipo-I com $M=1 / 10, V_{0}=1$, e $\hat{V}_{0}=1$ na (a) região I e (b) região II.

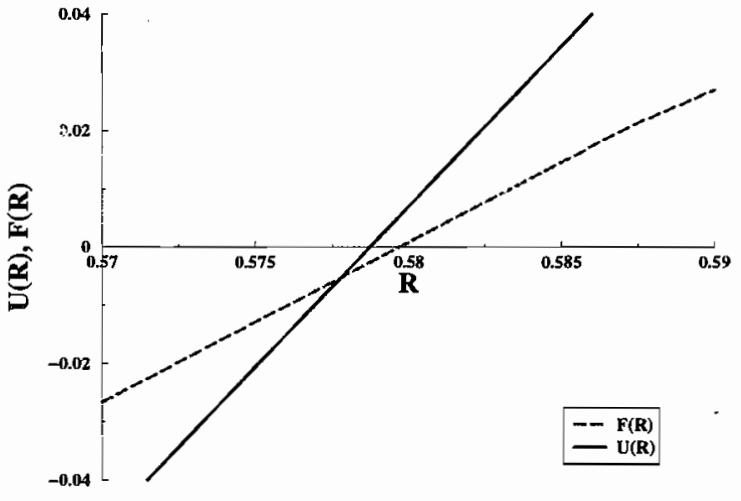

(a)

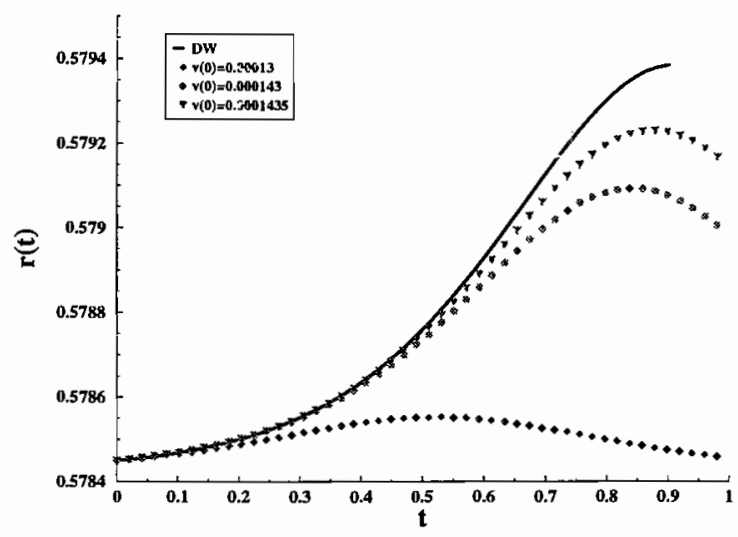

(b)

Figura 6.3: (a) Aproximação da região onde (6.12) é satisfeita, do gráfico de $U(R)$ e $F(R)$ com $\hat{\Lambda}<0$ e $M>0$ para as soluções tipo-I. (b) Movimento da parede de domínio e geodésicas para as soluções tipo-I com $M=1 / 10, V_{0}=-1$, e $\hat{V}_{0}=1$.

(4.50) tem solução somente quando $F(R) \leq 0$. Isto é automático somente se $U(R)<$ 0 , i.e., se $r$ é uma coordenada tipo tempo; portanto, devemos procurar por soluções com $U(R)>0$. De fato, ambas as condições

$$
F(R) \leq 0 \quad \text { e } \quad U(R)>0,
$$

podem coexistir em alguns casos como veremos no que segue. Para ilustrar os próximos exemplos, escolhemos $D=6$ dimensões. 


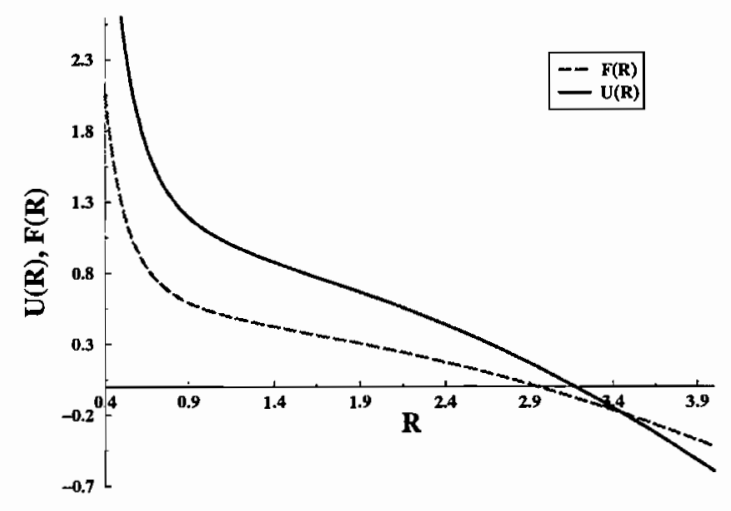

(a)

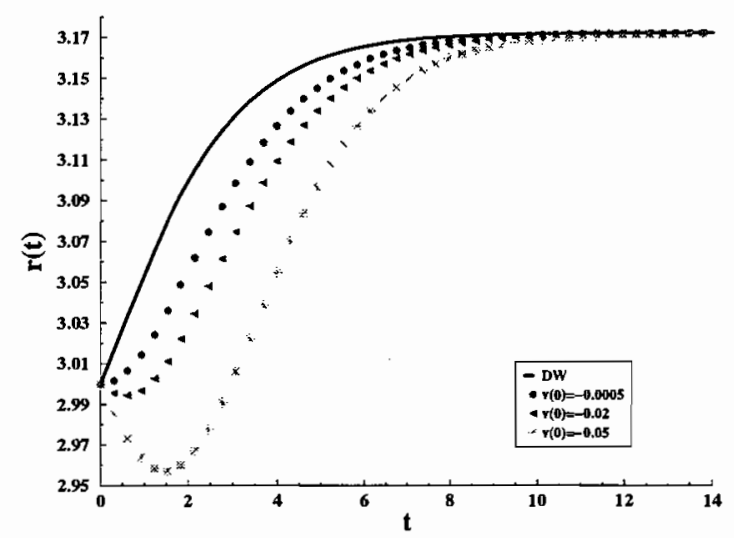

(b)

Figura 6.4: (a) $U(R)$ e $F(R)$ com $\hat{\Lambda}>0$ e $M<0$ para as soluções tipo-I. (b) Movimento da parede de domínio e geodésicas para $M=-1 / 10, V_{0}=1$, e $\hat{V}_{0}=1$.

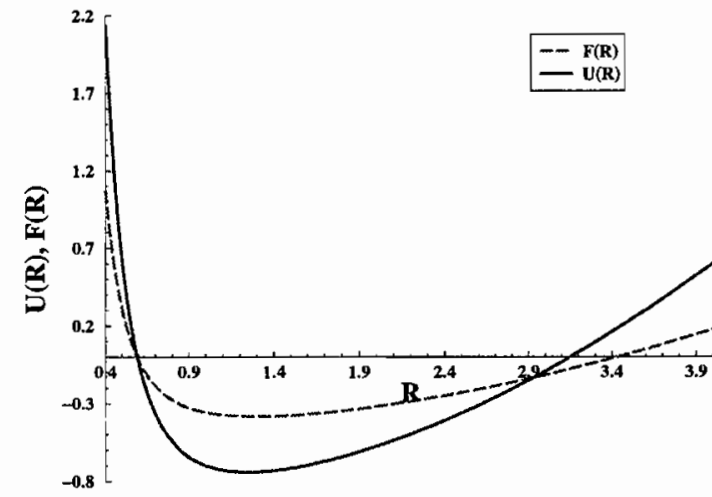

(a)

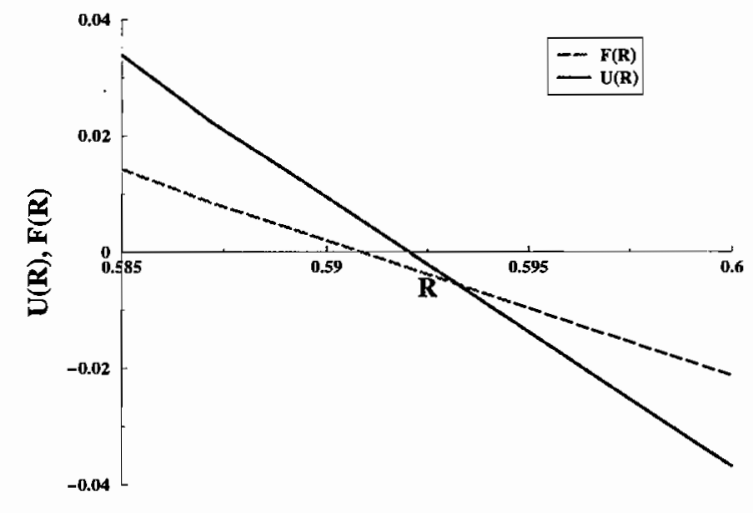

(b)

Figura 6.5: (a) $U(R)$ e $F(R)$ com $\hat{\Lambda}<0$ e $M=-1 / 10$ para as soluções tipo-I. (b) Aproximação da região do horizonte de eventos.

$\hat{\Lambda}>0, M>0$

Do gráfico de $U(R)$ (ver Fig. 6.1), podemos escolher a condição inicial para a parede de domínio, assumindo que (6.9) descreve um bulk dS-Schwarzschild com horizontes de eventos e cosmológico quando $M>0$ e $V_{0}>0$.

Assim, escolhemos a condição inicial para a parede de domínio dentro desta região, 
e onde $r$ é uma coordenada tipo espaço. Da Fig. 6.1 notemos que existem duas pequenas regiões, $r_{H} \leq r<0.593$ e $2.93 \leq r<r_{C}$, onde (6.12) é satisfeita. Os resultados são mostrados na Fig. 6.2. Vemos que para a região I, as geodésicas seguem a parede de domínio por um tempo e depois se desacoplam caindo dentro do horizonte de eventos. Para a região II, todas as geodésicas e a parede de domínio convergem ao horizonte cosmológico $r_{C}$, independentemente do valor de $\hat{V}_{0}$.

$\hat{\Lambda}<0, M>0$

Este caso descreve um bulk AdS-Schwarzschild. A condição (6.12) é satisfeita dentro de uma pequena região como podemos ver na Fig. 6.3(a). Porém, todas as geodésicas caem dentro do horizonte de eventos depois de seguir alguma trajetória na brana (ver Fig. 6.3(b)).

$\hat{\Lambda}>0, M<0$

Da Fig. 6.4(a), escolhemos a condição inicial para a equação de movimento da parede de domínio dentro da região onde (6.12) é satisfeita. Como podemos ver da Fig. 6.4(b), a parede de domínio e as geodésicas convergem ao horizonte cosmológico $r_{C}$. Porém, depois de uma velocidade inicial limiar, as geodésicas divergem à singularidade nua.

$\hat{\Lambda}<0, M<0$

Neste caso, (4.50) pode ser soluções somente quando $k=-1$. Este é um buraco negro topológico em um espaço assintoticamente Anti de Sitter. Da Fig. 6.5 vemos que não existe uma solução que satisfaça (6.12) entre os horizontes de eventos e cosmológico.

\subsubsection{Soluções Tipo-II}

Como vimos no capítulo 4 , as soluções tipo-II tem $\alpha=\beta / 2$ e $k=0$. A métrica é dada por

$$
U(r)=\left(1+b^{2}\right)^{2} r^{\frac{2}{\left(1+b^{2}\right)}}\left(-2 M r^{-\frac{\left(D-1-b^{2}\right)}{\left(1+b^{2}\right)}}-\frac{2 \Lambda}{\left(D-1-b^{2}\right)}\right),
$$

e o fator de escala é

$$
R(r)=r^{\frac{1}{\left(1+b^{2}\right)}}
$$

onde

$$
\Lambda=\frac{V_{0} e^{2 b \phi_{0}}}{D-2}
$$




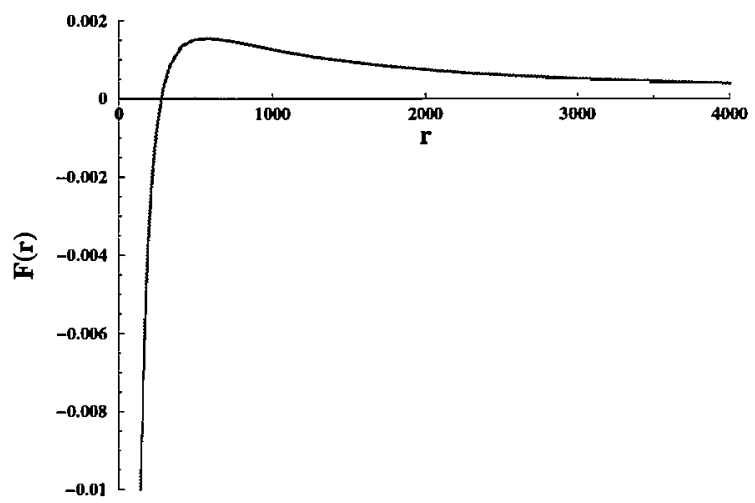

(a)

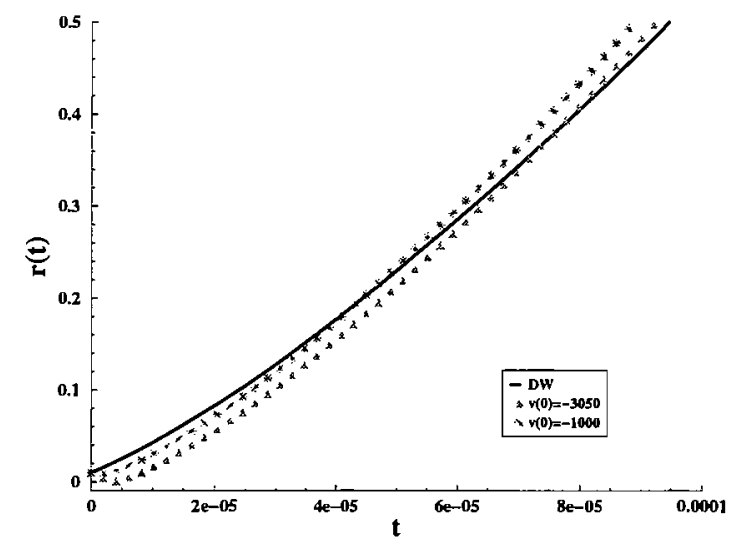

(b)

Figura 6.6: (a) $F(r) \operatorname{com} \hat{\Lambda}>0$ e $M<0$ para as soluções tipo-II, (b) movimento da parede de domínio e geodésicas para $V_{0}=1, \hat{V}_{0}=6, M=-10$, e $\beta=\sqrt{10}$.

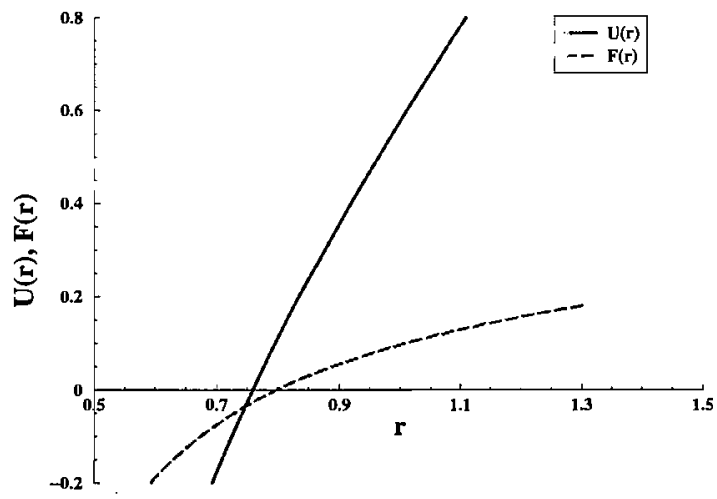

(a)

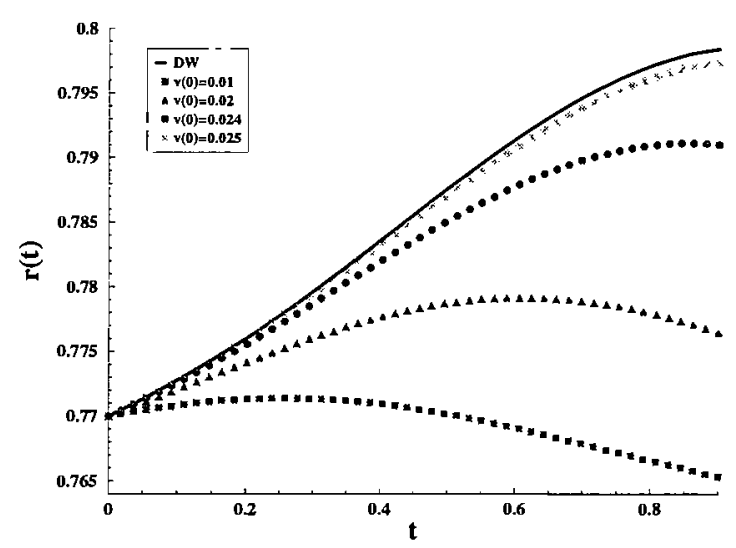

(b)

Figura 6.7: (a) $U(r)$ e $F(r)$ com $\hat{\Lambda}<0$ e $M>0$ para as soluções tipo-II, (b) movimento da parede de domínio e geodésicas para $V_{0}=-1, \hat{V}_{0}=1, M=1 / 10$, e $\beta=1 / \sqrt{2}$.

$$
b=\frac{1}{2} \beta \sqrt{D-2} .
$$

O potencial é dado pela expressão

$$
F(R)=-R^{2\left(1-b^{2}\right)}\left(M R^{-\left(D-1-b^{2}\right)}+\hat{\Lambda}\right),
$$




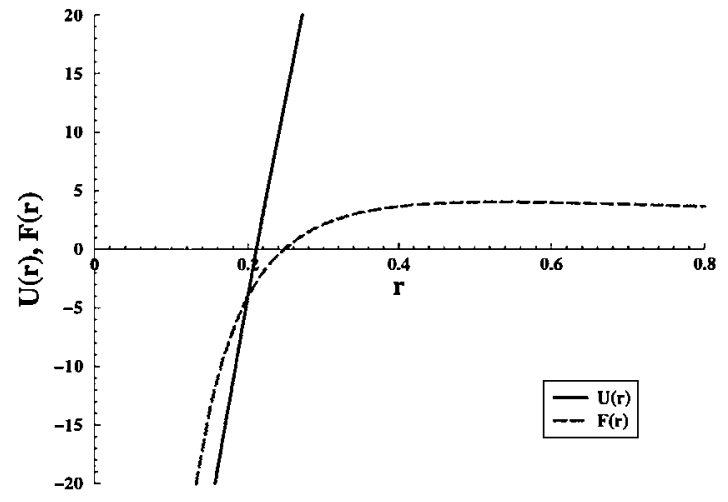

(a)

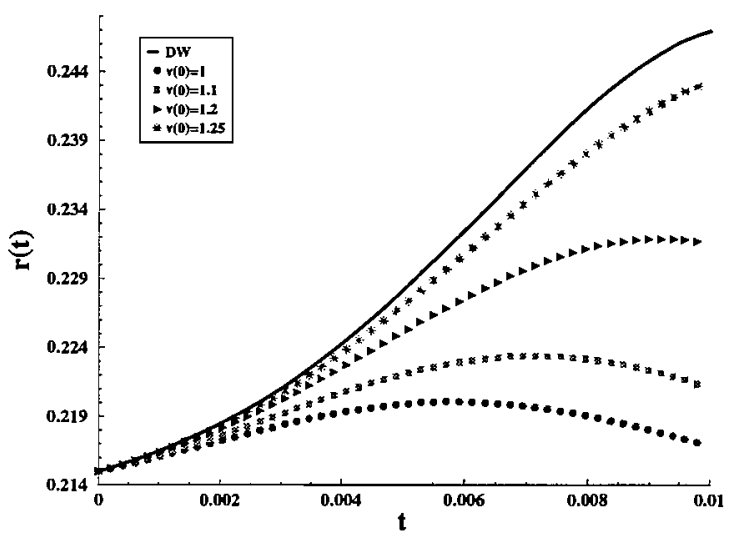

(b)

Figura 6.8: (a) $U(r)$ e $F(r)$ com $\hat{\Lambda}<0$ e $M>0$ para as soluções tipo-II, (b) movimento da parede de domínio e geodésicas para $V_{0}=-1, \hat{V}_{0}=1, M=10 \mathrm{e}$ $\beta=2$.

onde

$$
\hat{\Lambda}=\frac{e^{2 b \phi_{0}}}{D-2}\left(\frac{V_{0}}{D-1-b^{2}}+\frac{\hat{V}_{0}^{2}}{8(D-2)}\right) .
$$

Existem 12 casos para os quais escolhemos aqueles onde $r$ é uma coordenada espacial. Quando $b^{2}<D-1, r$ é uma coordenada espacial se $V_{0}<0$. Quando $b^{2}>D-1, r$ é tipo espaço se $M<0$.

Devemos também reescrever (6.12) como

$$
F(r) \leq 0 \quad \text { e } \quad U(r)>0 .
$$

$\hat{\Lambda}>0, M<0, b^{2}>D-1$

Neste caso, $U(r)$ é sempre positivo, enquanto $F(r)$ é negativo para $r$ pequeno. Da Fig. 6.6, vemos que alguns atalhos microscópicos aparecem no começo mesmo da solução e, depois de atravessar a brana, eles escapam para o infinito.

$\hat{\Lambda}<0, M>0, b^{2}<1$

Este caso descreve uma brana negra $(D-2)$-dimensional no espaço AdS. Aqui existe uma pequena região onde (6.19) é satisfeita, após o horizonte de eventos como podemos ver da Fig. 6.7. Mostramos a solução completa da parede de domínio e vemos 
que as geodésicas a seguem e depois se desacoplam para cair no horizonte de eventos em tempos posteriores.

$\hat{\Lambda}<0, M>0,1<b^{2}<D-1$

Este caso corresponde também a uma brana negra no espaço AdS. A região onde (6.19) é respeitada, é mostrada na Fig. 6.8. Como no caso anterior, todas as geodésicas seguem a parede de domínio e em tempos posteriores caem no horizonte de eventos.

$\hat{\Lambda}<0, M<0$

Como $F(r)$ é sempre positivo para todo $b^{2}$, não existem soluções para (6.6).

\subsubsection{Soluções Tipo-III}

Como foi visto no capítulo 4 , as soluções tipo-III têm $\alpha=2 / \beta(D-2)$. Neste caso a métrica é dada por

$$
U(r)=\left(1+b^{2}\right)^{2} r^{\frac{2}{\left(1+b^{2}\right)}}\left(-2 M r^{\frac{-\left[1+b^{2}(D-3)\right]}{\left(1+b^{2}\right)}}-\frac{2 \Lambda}{\left[1+b^{2}(D-3)\right]}\right),
$$

e o fator de escala é

$$
R(r)=\gamma r^{b^{2} /\left(1+b^{2}\right)}
$$

onde

$$
\gamma=\left(\frac{(D-3)}{2 k \Lambda\left(1-b^{2}\right)}\right)^{1 / 2} .
$$

Os valores de $\Lambda$ e $b$ são os mesmos que aqueles dados em (6.15) e (6.16).

O potencial $F(R)$ é

$$
\begin{aligned}
F(R)= & -\frac{(D-3) b^{4}}{2 k\left(1-b^{2}\right)\left(1+b^{2}(D-3)\right)}-M \gamma^{2} b^{4}\left(\frac{R}{\gamma}\right)^{-\left(D-3+1 / b^{2}\right)}- \\
& -\frac{\hat{V}_{0}^{2} e^{2 \phi_{0} / b} \gamma^{2}}{8(D-2)^{2}}\left(\frac{R}{\gamma}\right)^{-2\left(1 / b^{2}-1\right)}
\end{aligned}
$$

Se $V_{0}>0, r$ torna-se uma coordenada tipo tempo, enquanto que para $V_{0}<0$, é uma coordenada tipo espaço. Dos 12 casos mostrados em [56], escolhemos aqueles onde $r$ é uma coordenada espacial. Para todas estas soluções, a condição (6.19) é aplicável. 


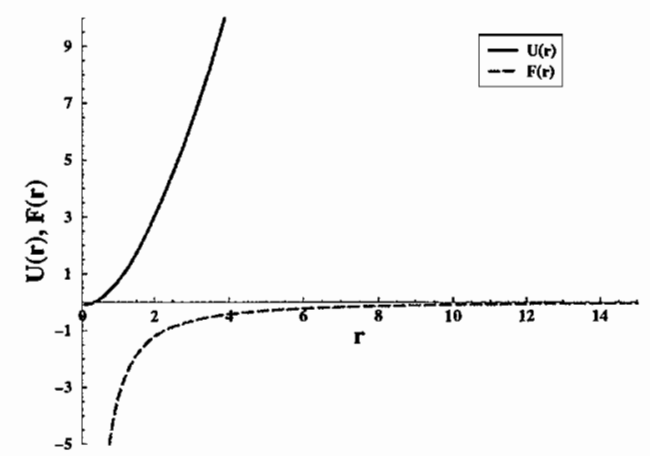

(a)

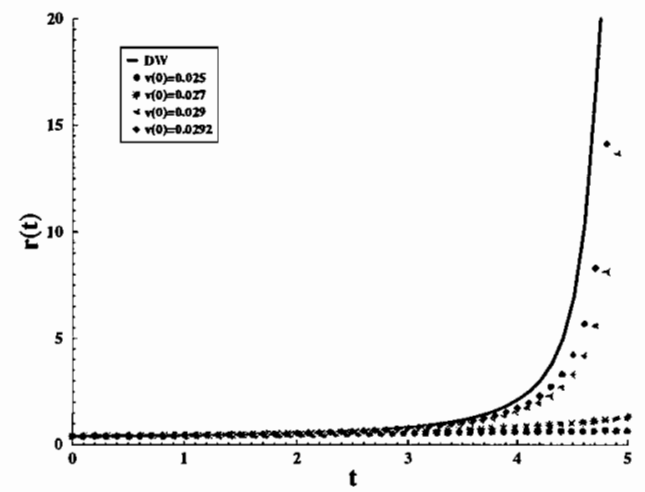

(b)

Figura 6.9: (a) $U(r)$ e $F(r)$ para as soluções tipo-III com $k=-1, M=1 / 10$ e $\beta^{2}<1 /(D-2)$. (b) Movimento da parede de domínio e geodésicas para $V_{0}=-1$, $\hat{V}_{0}=1, \phi_{0}=1$, e $\beta=1 / \sqrt{6}$.

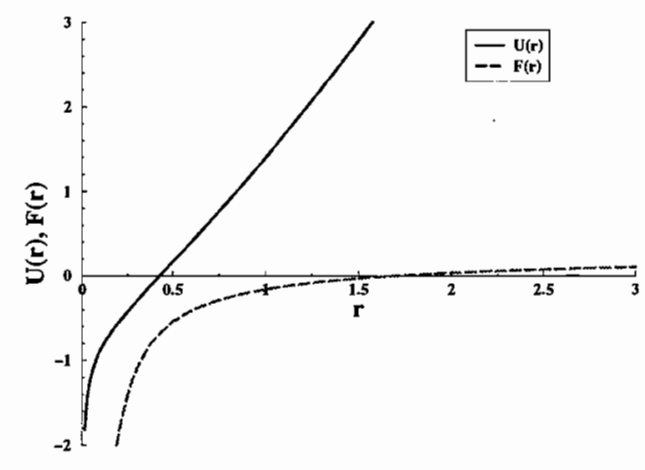

(a)

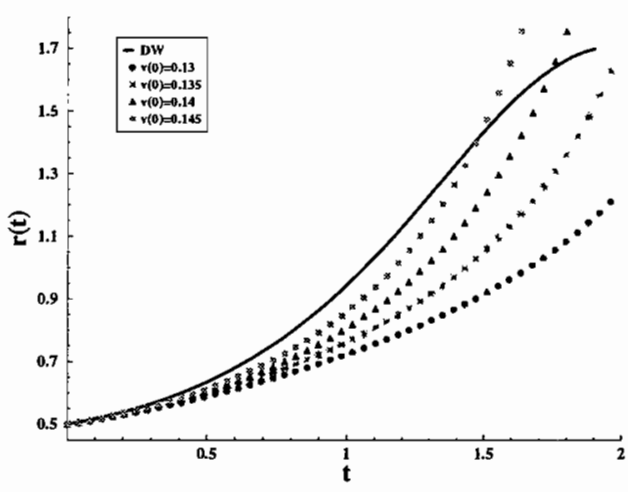

(b)

Figura 6.10: (a) $U(r)$ e $F(r)$ para $k=-1, M=1 / 10$ e $V_{0}<0$ nas soluções tipo-III, (b) movimento da parede de domínio e geodésicas para $V_{0}=-1, \hat{V}_{0}=1, \phi_{0}=1$, e $\beta=1 / \sqrt{2}$.

$V_{0}<0, M>0, b^{2}<1 /(D-1)$

Este caso descreve um buraco negro topológico no espaço AdS. Na Fig. 6.9, podemos ver a região onde (6.19) é satisfeita. Não existem atalhos neste intervalo; todas as geodésicas seguem a brana e depois ou divergem para o infinito ou caem no horizonte de eventos. 

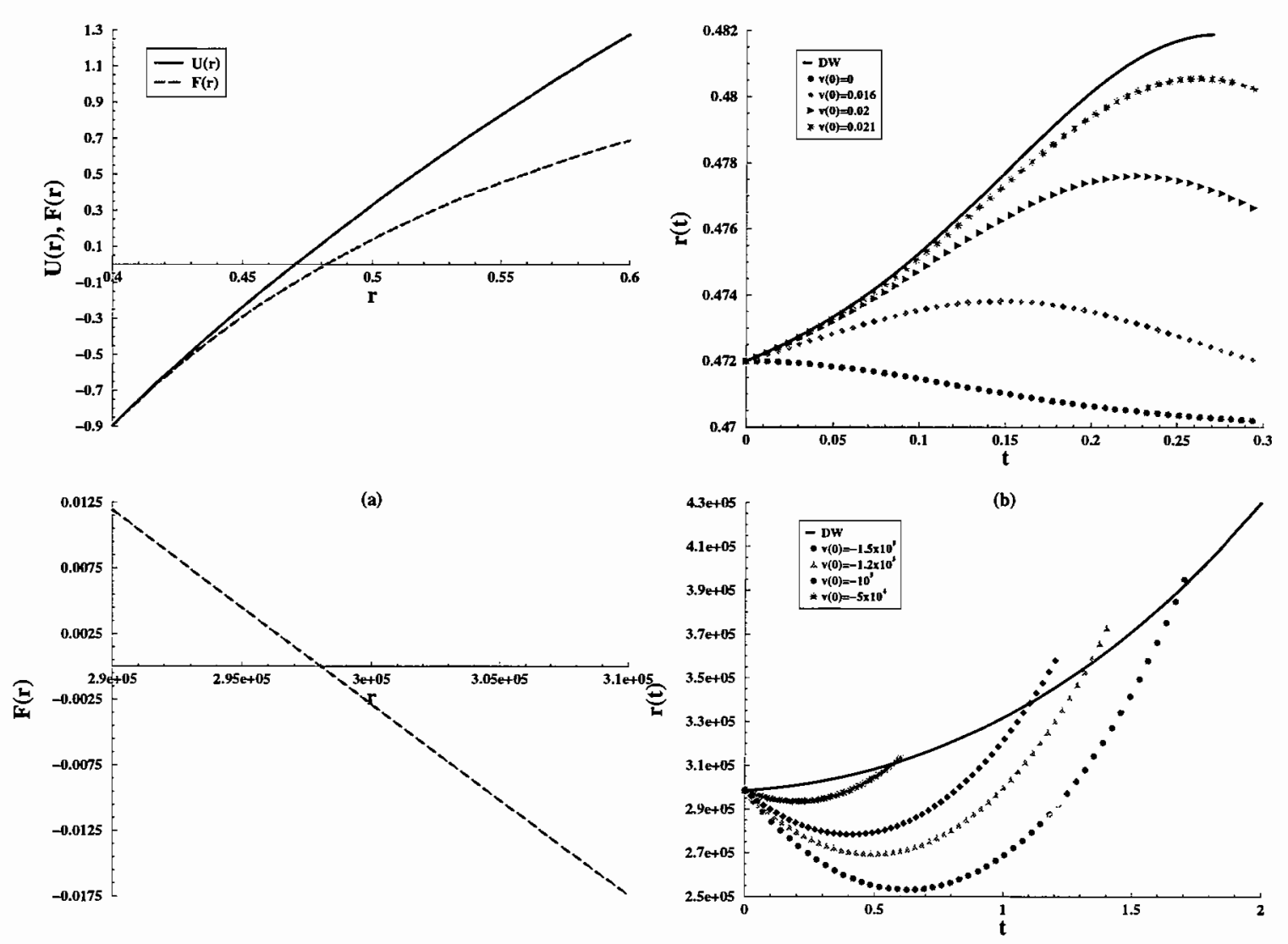

(c)

(d)

Figura 6.11: (a) $U(r)$ e $F(r)$ para $M=1 / 10$ e $V_{0}<0$ nas soluções tipo-III, (b) movimento da parede de domínio e geodésicas para $V_{0}=-1, \hat{V}_{0}=1, \phi_{0}=1$, e $\beta=\sqrt{5} / 2$, (c) $F(r)$ na região de interesse, (d) movimento da parede de domínio e geodésicas sob as mesmas condições que (b).

$V_{0}<0, M>0,1 /(D-1)<b^{2}<1$

Temos de novo um buraco negro topológico no espaço AdS. Existe um pequeno intervalo onde (6.6) tem solução, como podemos ver na Fig. 6.10(a). Nossos resultados são mostrados na Fig. 6.10(b). Note que a equação de movimento para a parede de domínio tem solução somente no intervalo mostrado. Isto quer dizer que só um grupo de geodésicas com velocidade inicial $\dot{r}(0)>v_{c}$, pode encontrar a parede de domínio depois de certa trajetória no bulk. É também mostrado que velocidades iniciais negativas forçam as geodésicas a cair dentro do horizonte de eventos. 


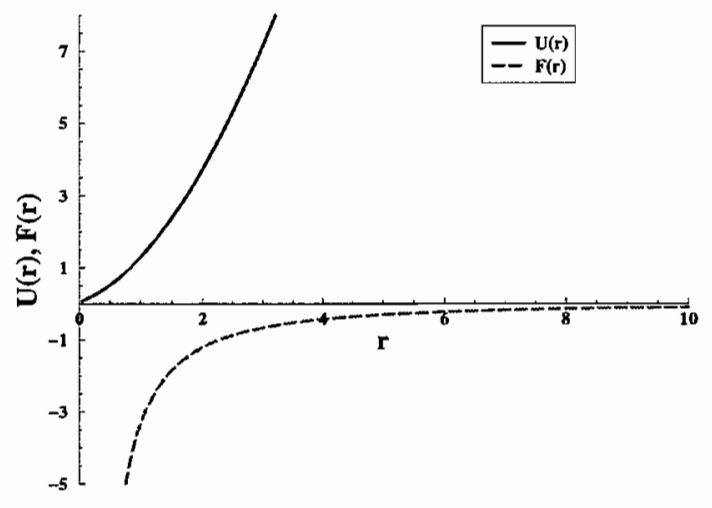

(a)

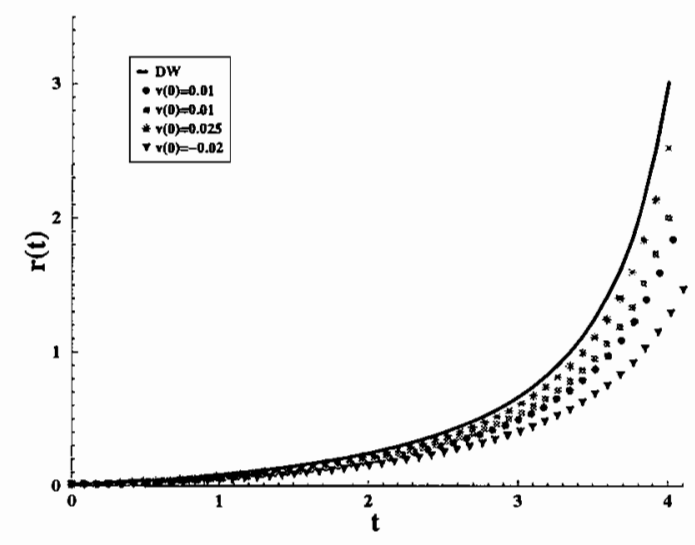

(b)

Figura 6.12: (a) $U(r)$ e $F(r)$ para as soluções tipo-III quando $k=-1, M=-1 / 10$, e $b^{2}<1 /(D-2)$, (b) movimento da parede de domínio e geodésicas para $V_{0}=-1$, $\hat{V}_{0}=1, \phi_{0}=1$, e $\beta=1 / \sqrt{6}$.

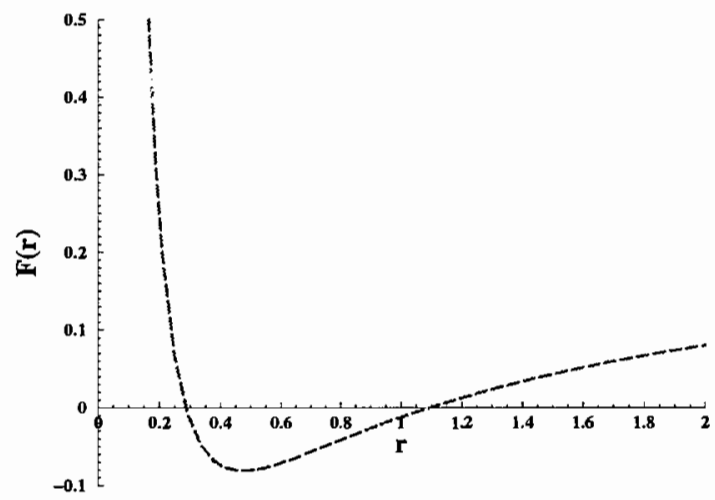

(a)

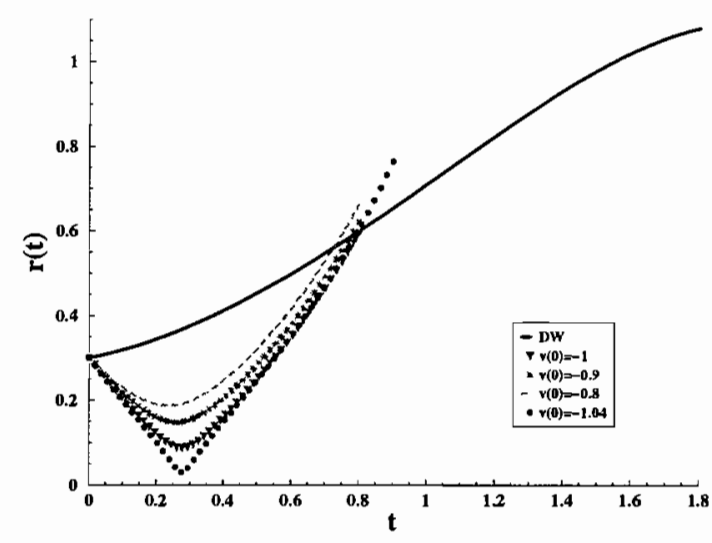

(b)

Figura 6.13: (a) $F(r)$ para as soluções tipo-III quando $k=-1, M=-1 / 10$, e $1 /(D-2)<b^{2}<1$, (b) movimento da parede de domínio e geodésicas para $V_{0}=-1$, $\hat{V}_{0}=1, \phi_{0}=1$, e $\beta=1 / \sqrt{2}$.

$V_{0}<0, M>0, b^{2}>1$

O buraco negro no espaço AdS aparecendo aqui tem seção espacial circular. Neste caso $U(r)$ é sempre positivo (então $r$ é sempre uma coordenada tipo espaço); porém, como devemos respeitar (6.19), devemos notar que $F(r) \leq 0$ para $r \geq 3 \times 10^{5}$. 


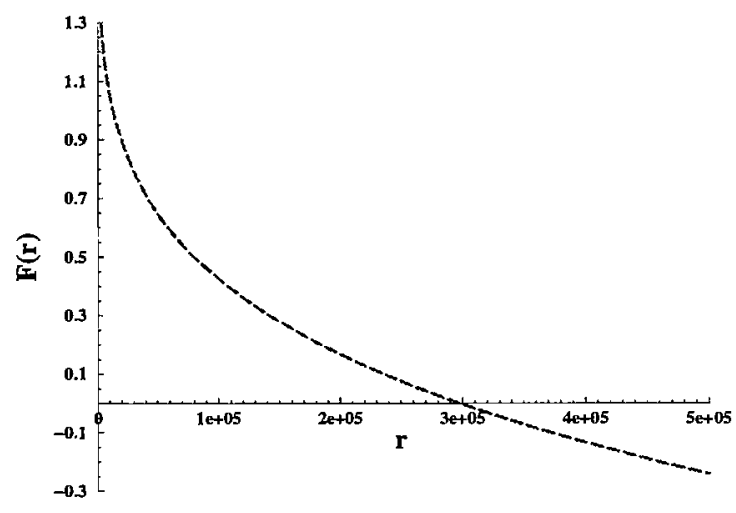

(a)

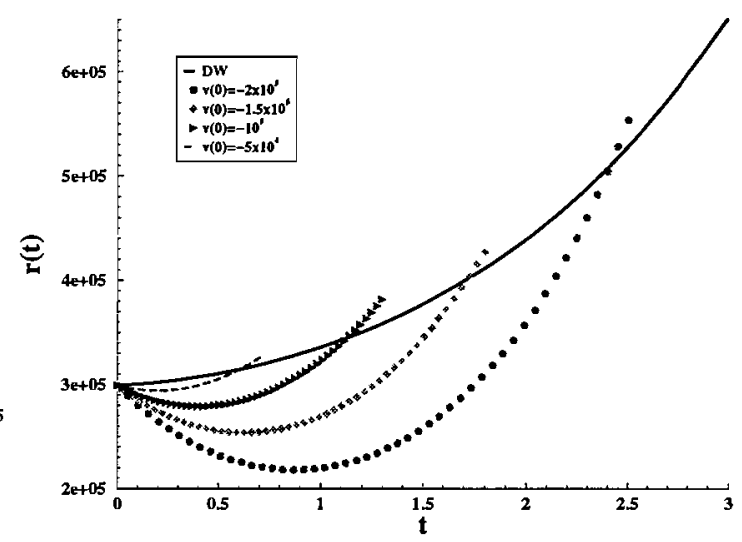

(b)

Figura 6.14: (a) $F(r)$ para as soluções tipo-III quando $M=-1 / 10$ e $b^{2}>1$, (b) movimento da parede de domínio e geodésicas para $V_{0}=-1, \hat{V}_{0}=1, \phi_{0}=1$, e $\beta=\sqrt{5} / 2$.

Encontramos que os atalhos são possíveis para várias velocidades iniciais se $M=0$.

O caso $M>0$ é mostrado na Fig. 6.11. Temos duas regiães de interesse depois do horizonte de eventos, dependendo somente no sinal de $F(r)$, já que $U(r)$ é positivo neste intervalo. $\mathrm{Na}$ primeira região, todas as geodésicas inicialmente seguem a brana e caem no horizonte de eventos em tempos posteriores. Na segunda região, temos atalhos novamente para diversas velocidades iniciais.

$V_{0}<0, M<0, b^{2}<1 /(D-1)$

Aqui $U(r)$ é sempre positivo, enquanto $F(r)$ é negativo no intervalo no intervalo mostrado na Fig. 6.12. A parede de domínio e as geodésicas divergem após algum tempo perto do fim do intervalo onde (6.6) tem solução.

$V_{0}<0, M<0,1 /(D-1)<b^{2}<1$

Neste caso $U(r)$ é sempre positivo, enquanto $F(r)$ é negativo para um intervalo pequeno, como pode ser visto na Fig. 6.13. Existem vários atalhos na região onde a equação de movimento da parede de domínio tem solução; porém, existe uma velocidade limiar após a qual as geodésicas não podem voltar. Como podemos ver da Fig. 6.13(b), a última curva mostrada aqui, [v(0) $=-1.04]$, não pode ser considerada um atalho real, já que não é uma solução contínua da equação geodésica, pelo contrário, representa uma transição entre os atalhos verdadeiros e as geodésicas caindo na sin- 
gularidade nua.

$V_{0}<0, M<0, b^{2}>1$

Agora $U(r)$ é sempre positivo e $F(r)$ irá determinar a condição inicial para a equação de movimento da parede de domínio. Como podemos ver da Fig. 6.14, vários atalhos aparecem.

\subsection{Tempo Próprio na Parede de Domínio e Atraso no Tempo}

O atraso no tempo entre o fóton viajando na parede de domínio e o gráviton viajando no bulk [40], pode ser calculado como segue. Já que os sinais cobrem a mesma distância

$$
\int \frac{d \tau_{\gamma}}{r\left(\tau_{\gamma}\right)}=\int \frac{d t_{g}}{r_{g}\left(t_{g}\right)} \sqrt{U\left(r_{g}\right)-\frac{\dot{r}_{g}(t)^{2}}{U\left(r_{g}\right)}}
$$

a diferença entre os tempos de vôo do fóton e do gráviiun, pode ser aproximadamente escrito como

$$
\frac{\Delta \tau}{r} \simeq \int_{0}^{\tau_{f}+\Delta \tau} \frac{d \tau_{\gamma}}{r\left(\tau_{\gamma}\right)}-\int_{0}^{\tau_{f}} \frac{\dot{\alpha} \tau_{g}}{r\left(\tau_{g}\right)}
$$

ou em termos do tempo do bulk

$$
\Delta \tau \simeq r\left(t_{f}\right) \int_{0}^{t_{f}} d t\left(\frac{1}{r_{g}(t)} \sqrt{U\left(r_{g}\right)-\frac{\dot{r}_{g}(t)^{2}}{U\left(r_{g}\right)}}-\frac{1}{r(t)} \frac{d \tau}{d t}\right) .
$$

O tempo transcorrido $t$, o correspondente tempo na parede de domínio $\tau$, e os atrasos (6.26), estão mostrados na Tabela 6.1 para todos os exemplos de atalhos mostrados neste capítulo. Note que $\Delta \tau<0$ para a última solução geodésica na Fig. 6.13 que, de fato, mostra que esta curva não pode ser considerada um atalho (provavelmente esteja caindo na singularidade nua).

\subsection{Discussão dos Resultados}

Consideramos novamente aqui a questão de atalhos em um universo descrito por uma brana imersa em um bulk com duas dimensões extras, a chamada parede de domínio, descrito pela gravitação de Einstein com um campo escalar. Em [56] o conjunto completo de soluções da equação de movimento da brana e as condições de DarmoisIsrael na parede foram obtidas. Estudamos a possibilidade de atalhos naqueles casos. 


\begin{tabular}{|c|c|c|c|}
\hline \multicolumn{4}{|c|}{ Atalhos tipo-II } \\
\hline Condições & $t$ & $\tau$ & $\Delta \tau$ \\
\hline Fig. 6.6, $v(0)=-3050$ & $7.5 \times 10^{-5}$ & $0 . \overline{0025}$ & 0.0466 \\
\hline Fig. $6.6, v(0)=-1000$ & $4.4 \times 10^{-5}$ & 0.0014 & 0.0027 \\
\hline \multicolumn{4}{|c|}{$\begin{array}{l}\text { Atalhos tipo-III } \\
\end{array}$} \\
\hline Fig. $6.10, v(0)=0.145$ & 1.49 & 0.970 & 0.210 \\
\hline Fig. $6.10, v(0)=0.140$ & 1.75 & 1.394 & 0.523 \\
\hline Fig. $6.11, v(0)=-5 \times 10^{4}$ & 0.58 & 353 & 3.51 \\
\hline Fig. $6.11, v(0)=-10^{5}$ & 1.11 & 683 & 26.6 \\
\hline Fig. $6.11, v(0)=-1.2 \times 10^{5}$ & 1.33 & 823 & 48.1 \\
\hline Fig. $6.11, v(0)=-1.5 \times 10^{5}$ & 1.69 & 1058 & 104 \\
\hline Fig. $6.13, v(0)=-0.8$ & 0.73 & 0.753 & 0.123 \\
\hline Fig. $6.13, v(0)=-0.9$ & 0.78 & 0.808 & 0.148 \\
\hline Fig. $6.13, v(0)=-1$ & 0.81 & 0.842 & 0.111 \\
\hline Fig. 6.13, $v(0)=-1.04$ & 0.80 & 0.831 & -0.129 \\
\hline$\overline{\text { Fig. } 6.14, v(0)=-5 \times 10^{4}}$ & 0.61 & 372 & 4.11 \\
\hline Fig. $6.14, v(0)=-10^{5}$ & 1.14 & 703 & 29.2 \\
\hline Fig. $6.14, v(0)=-1.5 \times 10^{5}$ & 1.72 & 1081 & 112 \\
\hline Fig. $6.14, v(0)=-2 \times 10^{5}$ & 2.42 & 1568 & 354 \\
\hline
\end{tabular}

Tabela 6.1: Tempo próprio na parede de dominio $\tau$, e atrasos no tempo $\Delta \tau$ para os atalhos aparecendo nas soluções tipo-II e tipo-III.

Como foi dito em [40], onde é considerado o caso de um universo dinâmico, os atalhos podem fornecer uma explicação alternativa ao problema do horizonte.

Certamente, se os atalhos gravitacionais forem comuns antes da nucleosíntese em um modelo realista, eles podem fornecer uma alternativa à inflação para termalizar o universo primitivo. No modelo tratado neste capítulo, onde nosso universo é substituído por uma parede de domínio, provamos que os atalhos podem existir e, sobre tudo, eles são abundantes, que é uma condição necessária para resolver o problema de homogeneidade.

Porém, isto não é suficiente e mais considerações em um cenário mais realístico precisam ser levadas em conta. Devemos também fazer notar que um dílaton que varia com o tempo poderia gerar uma variação detectável da constante de Newton, o que impõe certas restrições nos parâmetros do presente modelo.

No entanto, apesar das suas deficiências, o modelo apresenta resultados interessantes. Além disso, mostramos que o atraso no tempo de vôo dentro da brana pode chegar a ser comparável com o tempo de vôo do próprio gráviton. Isto empresta maior apoio para uma termalização por meio de intercâmbio de grávitons através das 
dimensões extras, ainda que não seja uma prova da solução do problema. 


\section{Capítulo 7}

\section{Dinâmica não Linear de uma Brana em um Modelo de 6 Dimensões}

No presente capítulo, vamos considerar o modelo de brana dinâmica em um espaçotempo 6-dimensional contendo uma singularidade, discutido na seção 4.4, onde usando as condições de Darmois-Israel, derivamos o sistema de equações que governa o movimento da 4-brana imersa neste cenário desde o ponto de vista de um observador no bulk [44]. Aqui, analisaremos o comportamento da srana quando sua posição é perturbada ao redor de um ponto fixo e, logo, resolveremos a dinâmica não linear completa nos diferentes cenários resultantes da combinação dos parâmetros aparecendo no modelo. Também investigaremos a existência de ataihos gravitacionais e calcularemos o atraso entre os sinais do gráviton e do fóton, assim como a razão entre os correspondentes horizontes subtendidos.

\subsection{O Modelo, a Equação Geodésica e o Atraso do Tempo}

Consideramos um modelo em 6 dimensões descrito pela métrica mostrada na seção 4.4

$$
d s^{2}=-h(z) d t^{2}+a^{2}(z) d \Sigma_{k}^{2}+h^{-1}(z) d z^{2},
$$

onde

$$
\begin{gathered}
a(z)=\frac{z}{l} \\
d \Sigma_{k}^{2}=\frac{d r^{2}}{1-k r^{2}}+r^{2} d \Omega_{(2)}^{2}+\left(1-k r^{2}\right) d y^{2}
\end{gathered}
$$

e

$$
h(z)=k+\frac{z^{2}}{l^{2}}-\frac{M}{z^{3}}+\frac{Q^{2}}{z^{6}}
$$


com $l^{-2} \propto-\Lambda$, e $M$ e $Q^{2}$ constantes. Devemos notar também que $y$ é uma coordenada compactificada.

Esta métrica contém uma singularidade em $z=0$. Lembramos que (7.1) é valida nas partes $z<\mathcal{R}(t)$ de superfícies de $t$ constante, e sua reflexão, pela simetria de espelho, é valida nas partes $z>\mathcal{R}(t)$.

Com este Ansatz, as condições de Darmois-Israel (4.78) se reduzem a somente duas equações,

$$
\begin{aligned}
\ddot{\mathcal{R}}+\frac{1}{2} \frac{h^{\prime}}{h^{3}} \dot{\mathcal{R}}^{4}-3 \frac{h^{\prime}}{h} \dot{\mathcal{R}}^{2}+\frac{1}{2} h h^{\prime} & =-\kappa_{(6)}^{2}\left(\frac{3 \rho+4 p}{8}\right) h^{2}\left(1-\frac{\dot{\mathcal{R}}^{2}}{h^{2}}\right)^{3 / 2} \\
\frac{a^{\prime}}{a}+\frac{\dot{\mathcal{R}}}{h^{2}} \frac{\dot{a}}{a} & =\kappa_{(6)}^{2} \frac{\rho}{8}\left(1-\frac{\dot{\mathcal{R}}^{2}}{h^{2}}\right)^{1 / 2}
\end{aligned}
$$

onde todos os coeficientes da métrica devem ser calculados na brana. O sistema (7.5) descreve a dinâmica não linear completa da brana imersa no bulk estático (7.1).

Consideramos dois pontos na brana $r_{A}$ e $r_{B}$. Em geral existe mais de una geodésica conectando estes pontoś no espaço-tempo $(1+5)$-dimensional. As tıajetórias dos fótons devern estar na brana e aquelas dos grávitons podem estar no bulk. A equação da trajetória do gráviton é definida igualando (7.1) a zero. Já que estamos procurando por uma trajetória que minimiza $t$ quando o ponto final $r_{B}$ está na brana, o problema se reduz ao problema de Euler-Lagrange [33]. Assim, como dito no capítulo 4, o caminho mais curto para o gráviton é dado por [34, 43]

$$
\ddot{\mathcal{R}}_{g}+\left(\frac{1}{\mathcal{R}_{g}}-\frac{3}{2} \frac{h^{\prime}}{h}\right) \dot{\mathcal{R}}_{g}^{2}+\frac{1}{2} h h^{\prime}-\frac{h^{2}}{\mathcal{R}_{g}}=0 .
$$

Podemos também calcular o atraso no tempo entre o fóton viajando na brana e os grávitons viajando no bulk. Já que ambos os sinais cobrem a mesma distância [40],

$$
\int_{0}^{\tau_{f}+\Delta \tau} \frac{d \tau_{\gamma}}{\mathcal{R}\left(\tau_{\gamma}\right)}=\int_{0}^{t_{f}} \frac{d t_{g}}{\mathcal{R}_{g}\left(t_{g}\right)} \sqrt{h\left(\mathcal{R}_{g}\right)-\frac{\dot{\mathcal{R}}_{g}(t)^{2}}{h\left(\mathcal{R}_{g}\right)}},
$$

a diferença entre os tempos de vôo do fóton e do gráviton medido por um observador na brana, pode ser aproximadamente escrito em termos do tempo do bulk $t$ como [43]

$$
\Delta \tau \simeq \mathcal{R}\left(t_{f}\right) \int_{0}^{t_{f}} d t\left(\frac{1}{\mathcal{R}_{g}(t)} \sqrt{h\left(\mathcal{R}_{g}\right)-\frac{\dot{\mathcal{R}}_{g}(t)^{2}}{h\left(\mathcal{R}_{g}\right)}}-\frac{1}{\mathcal{R}(t)} \frac{d \tau}{d t}\right) .
$$


É também interessante olhar para a razão entre os horizontes subtendidos pelos fótons viajando na brana e pelos grávitons viajando no bulk. Esta razão usa as mesmas quantidades previamente mencionadas para o atraso do tempo,

$$
\frac{g}{\gamma}=\frac{\int_{0}^{t_{f}} \frac{d t}{\mathcal{R}_{g}(t)} \sqrt{h\left(\mathcal{R}_{g}\right)-\frac{\dot{\mathcal{R}}_{g}(t)^{2}}{h\left(\mathcal{R}_{g}\right)}}}{\int_{0}^{t_{f}} \frac{d t}{\mathcal{R}(t)} \frac{d \tau}{d t}}
$$

\subsection{Flutuações na Posição da Brana}

Nesta seção, estudamos perturbativamente o comportamento da brana em um ponto fixo. Definimos a solução de fundo como o caso onde a posição da brana está congelada, i.e.

$$
z=\mathcal{R}(t)=\overline{\mathcal{R}}=\text { const. } .
$$

Desta forma, o sistema (7.5) se reduz a

$$
\begin{aligned}
\frac{1}{2} \frac{h^{\prime}}{h} & =-\frac{\kappa_{(6)}^{2}}{8}(3 \bar{\rho}+4 \bar{p}) \\
\frac{a^{\prime}}{a} & =\frac{\kappa_{(6)}^{2}}{8} \bar{\rho} .
\end{aligned}
$$

As equações de movimento para as flutuações na posição da brana pode ser obtida linearizando as equações de movimento exatas (7.5), ao redor de uma configuração de equilíbrio, isto é,

$$
\begin{aligned}
\frac{\delta \ddot{\mathcal{R}}}{h^{2}}+\frac{1}{2} \delta\left(\frac{h^{\prime}}{h}\right) & =-\frac{\kappa_{(6)}^{2}}{8}(3 \delta \rho+4 \delta p) \\
\delta\left(\frac{a^{\prime}}{a}\right) & =\frac{\kappa_{(6)}^{2}}{8} \delta \rho .
\end{aligned}
$$

Das expressões explícitas de $h(\operatorname{com} Q=0)$ e $a$, podemos achar

$$
\begin{aligned}
\delta\left(\frac{a^{\prime}}{a}\right) & =-\frac{\kappa_{(6)}^{4}}{64} \bar{\rho}^{2} \delta \mathcal{R} \equiv m_{a}^{2} \delta \mathcal{R} \\
\frac{1}{2} \delta\left(\frac{h^{\prime}}{h}\right) & =\left\{-\frac{\kappa_{(6)}^{4}}{32}(3 \bar{\rho}+4 \bar{p})^{2}+\frac{\frac{1}{l^{2}}-6 \frac{M}{\overline{\mathcal{R}}^{5}}}{k+\frac{\overline{\mathcal{R}}^{2}}{l^{2}}-\frac{M}{\overline{\mathcal{R}}^{3}}}\right\} \delta \mathcal{R} \equiv m_{h}^{2} \delta \mathcal{R} .
\end{aligned}
$$

Como podemos ver de (7.13), as flutuações na posição da brana estão ligadas às flutuações na matéria. No entanto, quando temos o caso de flutuações de matéria adiabáticas

$$
\delta p=v_{s}^{2} \delta \rho,
$$


podemos derivar uma equação para as flutuações na posição da brana com uma apropriada combinação linear de (7.13)

$$
\frac{\delta \ddot{\mathcal{R}}}{h^{2}}+\left(m_{h}^{2}+\left(3+4 v_{s}^{2}\right) m_{a}^{2}\right) \delta \mathcal{R}=0
$$

enquanto que as flutuações na matéria estarão relacionada a aquelas na posição da brana por

$$
\frac{\kappa_{(6)}^{2}}{8} \delta \rho=m_{a}^{2} \delta \mathcal{R} .
$$

Assumindo uma equação de estado $p=\omega \rho$, (7.17) pode finalmente ser escrita como

$$
\frac{\delta \ddot{\mathcal{R}}}{h^{2}}+\left\{\frac{\frac{1}{l^{2}}-6 \frac{M}{\mathcal{R}^{5}}}{k+\frac{\overline{\mathcal{R}}^{2}}{l^{2}}-\frac{M}{\mathcal{R}^{3}}}-(7+8 \omega)(3+4 \omega) \frac{\kappa_{(6)}^{4}}{64} \bar{\rho}^{2}\right\} \delta \mathcal{R}=0 .
$$

A constante $M$ pode ser expressa em termos de $\overline{\mathcal{R}}$ e $\omega$ das condições de Darmois-Israel para uma brana estática [34], combinando (5.6) e (5.7):

$$
M=\frac{2 \overline{\mathcal{R}}^{3}}{8 \omega+3}\left\{(4 \omega+3) k+4(\omega+1) \frac{\overline{\mathcal{R}}^{2}}{l^{2}}\right\} .
$$

No caso de uma parede de domínio em um bulk plano $(k=0, \omega=-1)$, o termo dentro de chaves em (7.19) pode ser interpretado como uma constante cosmológica. efetiva na brana,

$$
\frac{1}{\overline{\mathcal{R}}^{2}}-\frac{\kappa_{(6)}^{4}}{64} \bar{\rho}^{2} \equiv-\Lambda_{e f f},
$$

que é nula quando levamos em conta (7.12). Isto corresponde ao ajuste fino de Randall e Sundrum $[7,8]$ em 6 dimensões. Assim, a constante cosmológica efetiva na brana é zero.

Portanto, ficamos com a equação de movimento para um campo escalar livre como em 5 dimensões $[57,58]$. Neste caso a brana está em repouso e a posição de "equilíbrio" $\overline{\mathcal{R}}$ pode ser escolhida arbitrariamente. Porém, um pequeno desvio desta posição resulta em um comportamento de fuga.

Este resultado é compatível com a solução numérica do sistema não linear completo (7.5), como veremos na próxima seção.

\subsection{Dinâmica Não Linear da Brana}

O sistema (7.5) descrevendo a dinâmica da brana pode ser resolvido numericamente para várias combinações de $M, Q^{2}, k$ e $l^{2}$. No apêndice $\mathrm{D}$ mostramos um exemplo da obtenção em MAPLE dos gráficos deste capítulo. Quando $M, Q^{2}$ e $k$ se anulam, a 

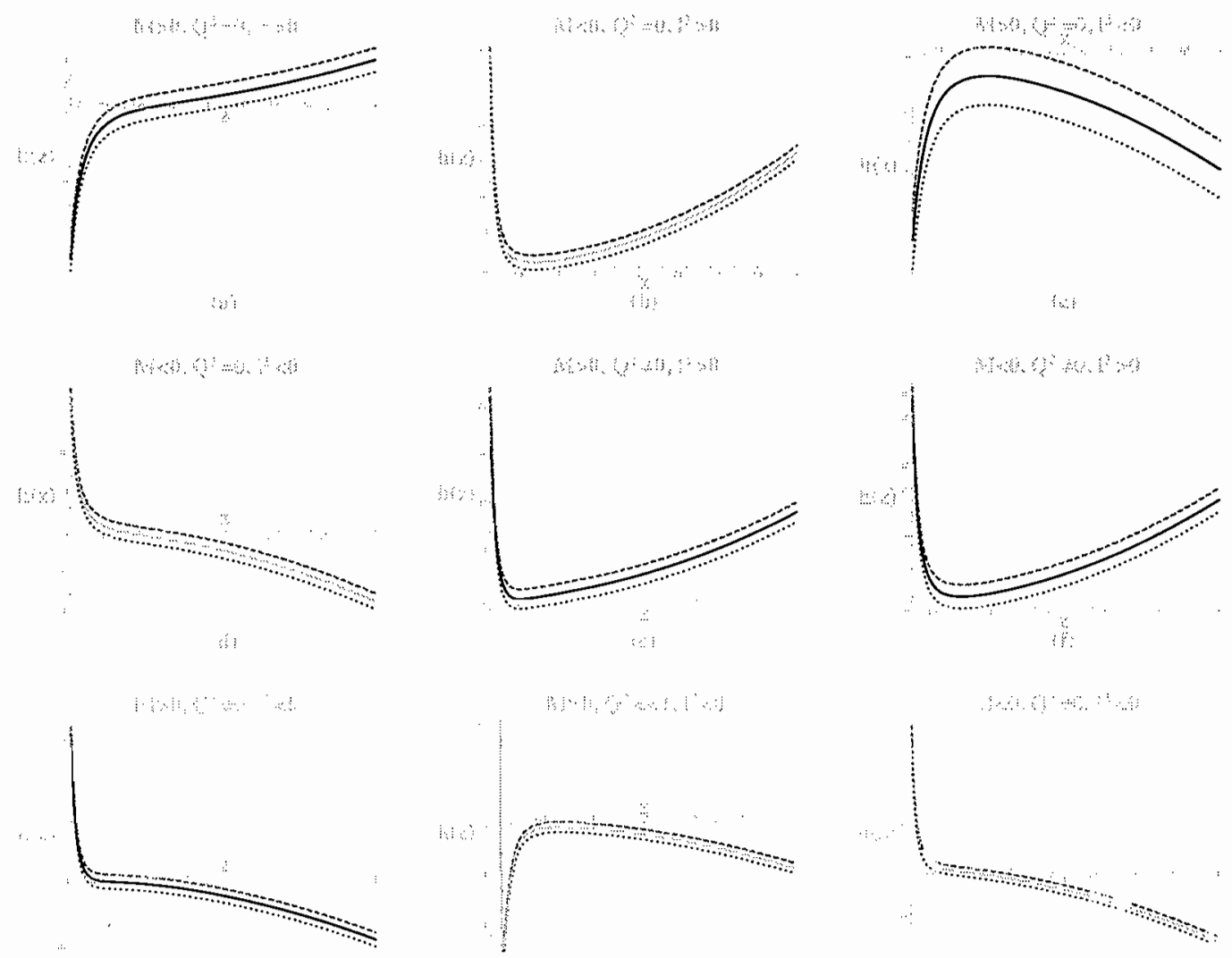

Figura 7.1: $h(z)$ para todas as possíveis combinações de $M, Q^{2}$ e $l^{2}$, incluindo os casos $k=1$ (linhas entrecortadas), $k=0$ (linhas sólidas) e $k=-1$ (linhas pontilhadas).

solução para $\mathcal{R}(t)$ é uma constante ou uma função linear em $t$ dependendo da condição inicial dada para $\dot{\mathcal{R}}$, e verificamos o comportamento achado através de flutuações na posição da brana.

Para $M$ e $Q^{2}$ não nulos, resolvemos (7.5) nos casos típicos de uma parede de domínio $(\omega=-1)$, uma brana dominada por matéria $(\omega=0)$ e uma brana dominada por radiação $(\omega=1 / 3)$. Na Fig. 7.1 mostramos todas as possíveis formas de $h(z)$, devido às combinações de $M, Q^{2}, k$ e $l^{2}$, que estudamos nesta seção. Alguns dos nossos resultados são também ilustrados no que segue, junto com a solução da equação geodésica (7.6), para verificar a possibilidade de se ter atalhos. Também calculamos os atrasos no tempo e a razão entre os horizontes do gráviton e do fóton para os exemplos de atalhos aparecendo neste trabalho, e são mostrados na Tabela 7.1, conjuntamente com o tempo de vôo do gráviton no bulk e seu correspondente tempo na brana de acordo com a Eq. (4.68). 
Classificamos todos os casos de acordo com o sinal do parâmetro $M$, e se estamos nos bulks dS ou AdS. Da mesma maneira, estudamos o caso do buraco negro sem carga, assim como as soluções tipo Reissner-Nordström, ou seja, 8 casos em total.
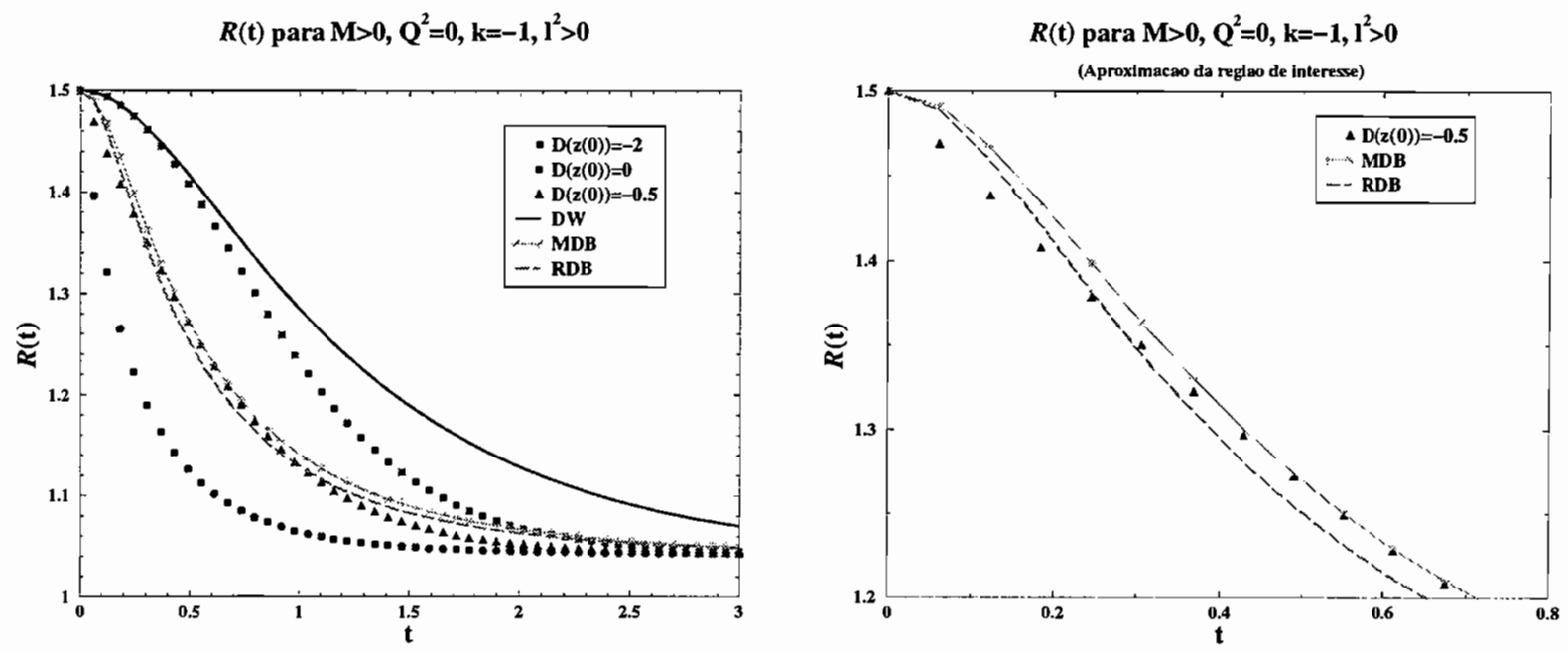

Figura 7.2: Evolução do fator de escala para uma parede de domínio e branas dominadas por matéria e radiação, e geodésicas quando $M>0, Q^{2}=0$ e $l^{2}>0$.

$R(\mathrm{t})$ para $\mathrm{M}<0, \mathrm{Q}^{2}=0, \mathrm{k}=1, \mathrm{I}^{2}>0$

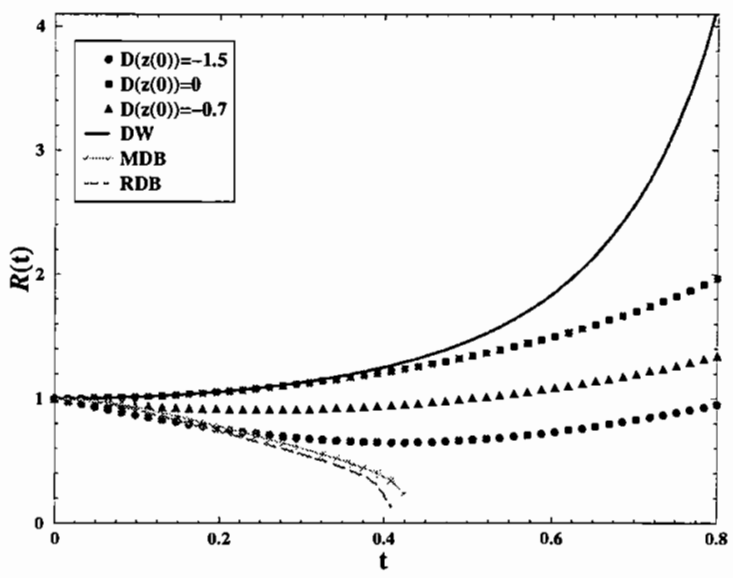

$R(t)$ para $M<0, Q^{2}=0, k=1, I^{2}>0$

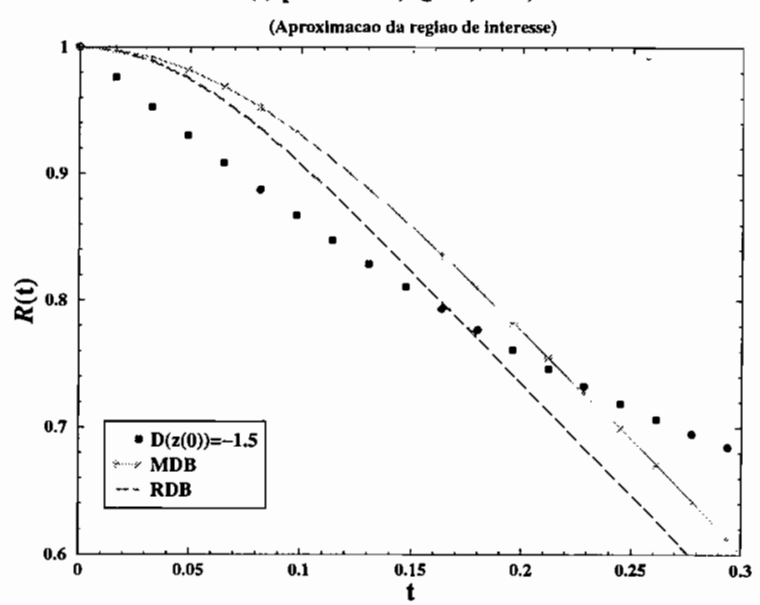

Figura 7.3: Evolução do fator de escala para uma parede de domínio e branas dominadas por matéria e radiação, e geodésicas quando $M<0, Q^{2}=0$ e $l^{2}>0$.

Primeiro consideremos o caso com carga zero. 
$R(t)$ para $M>0, Q^{2}=0, k=1,1^{2}<0$

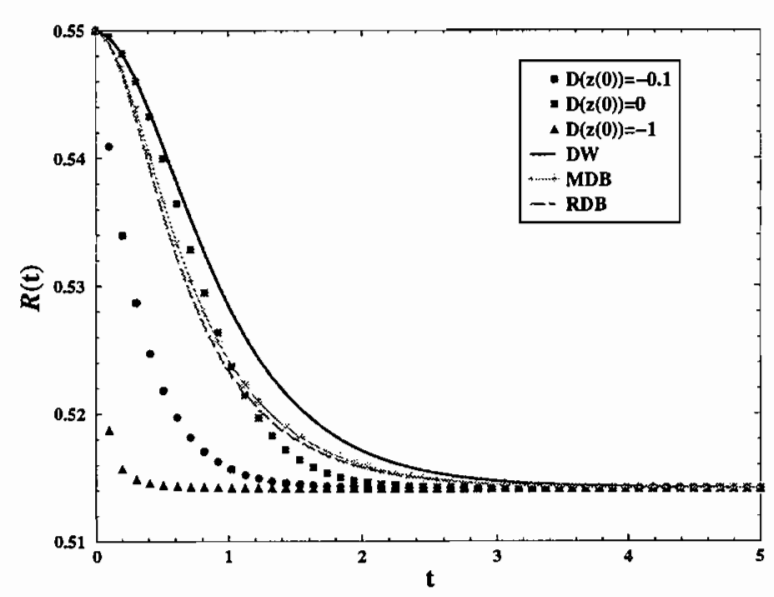

$R(\mathrm{t})$ para $\mathrm{M}>0, \mathrm{Q}^{2}=0, \mathrm{k}=1, \mathrm{I}^{2}<0$

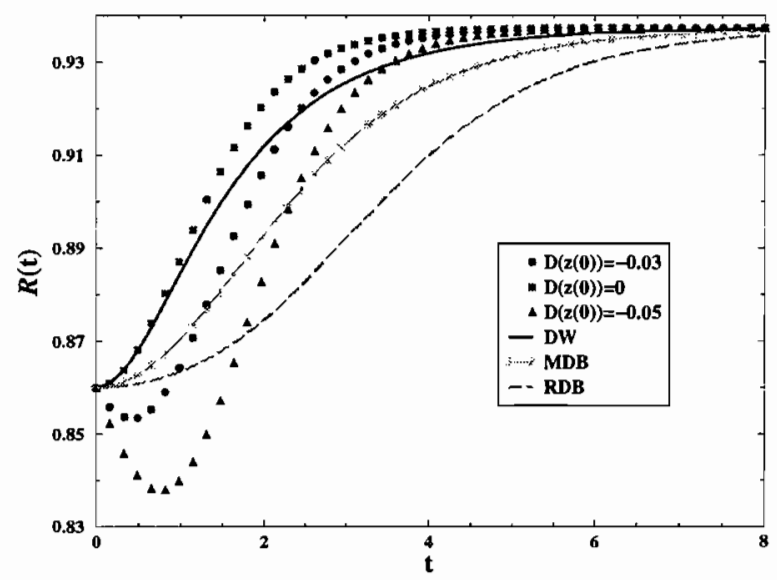

Figura 7.4: Evolução do fator de escala para uma parede de domínio e branas dominadas por matéria e radiação, e geodésicas quando $M>0, Q^{2}=0$ e $l^{2}<0$.
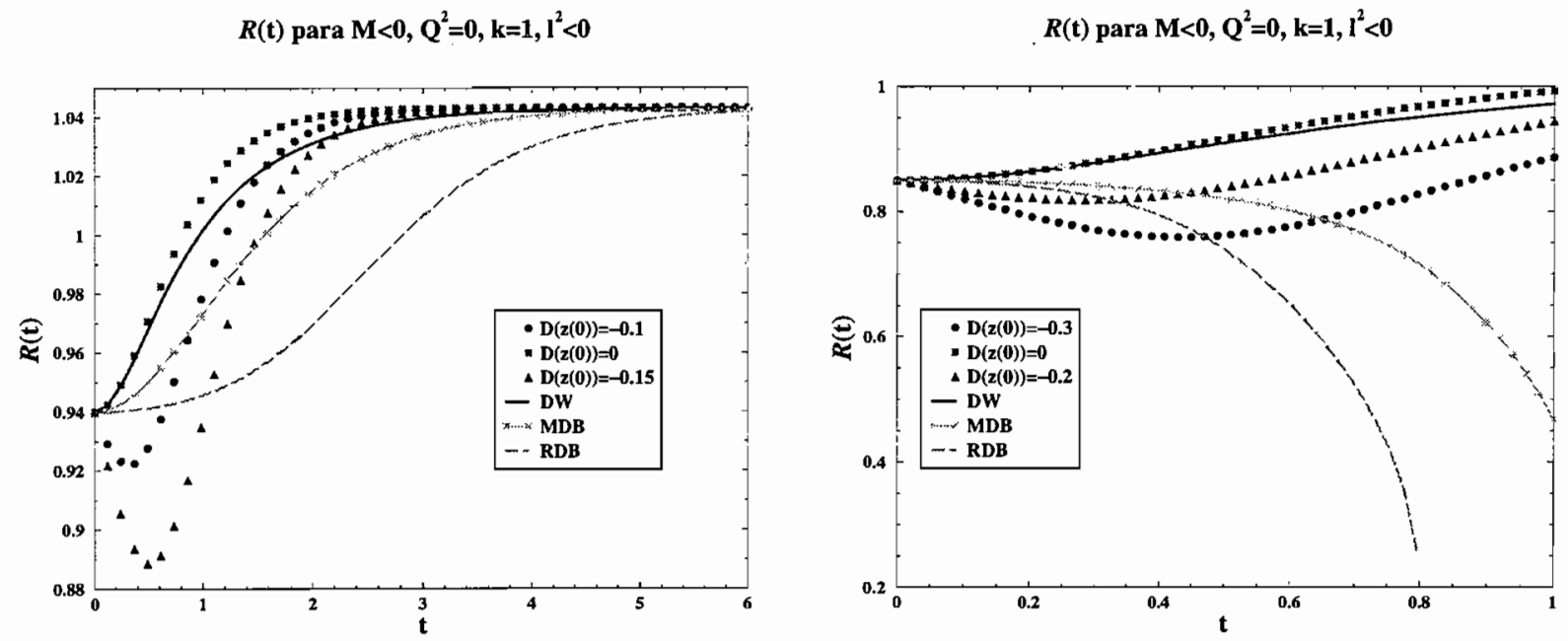

Figura 7.5: Evolução do fator de escala para uma parede de domínio e branas dominadas por matéria e radiação, e geodésicas quando $M<0, Q^{2}=0$ e $l^{2}<0$.

\subsection{1 $M>0, Q^{2}=0, l^{2}>0$}

Para $M$ e $l^{2}$ positivos, quando $k=1$, o bulk é Anti de Sitter-Schwarzschild, enquanto que para $k \neq 1$ temos um buraco negro "topológico" com horizonte de eventos plano ou hiperbólico em um espaço assintoticamente Anti de Sitter. Este caso é mostrado 
na Fig. 7.1(a).

Para uma parede de domínio $(\omega=-1)$ e $k=1$, a brana cai dentro do horizonte de eventos $r_{H}$ quando a condição inicial para a posição da brana está perto de $r_{H}$; de outra forma, $\mathcal{R}(t)$ cresce. De outro lado, para $k \neq 1$ a brana sempre cai no horizonte de eventos $r_{H}$.

No caso de uma brana dominada por matéria $(\omega=0)$, a brana cai dentro do horizonte de eventos para qualquer $k$. Este comportamento é também verificado para uma brana dominada por radiação $(\omega=1 / 3)$.

Vejamos agora o comportamento das geodésicas. Quando $k=1 \mathrm{e}$ a condição inicial na brana é escolhida perto de $r_{H}$, as geodésicas caem no horizonte de eventos. Algumas delas seguem a parede de domínio no início, enquanto outras deixam as branas dominadas por matéria e radiação, retornando depois de um tempo curto (atalhos) e novamente deixam as branas para cair na singularidade. De outro lado, se a condição inicial é tomada longe do horizonte de eventos, as geodésicas crescem acompanhando a parede de domínio inicialmente, mas seu crescimento é lento; no entanto, elas nunca retornam à parede depois do desacoplamento. Quando $k \neq$ 1 , independentemente da condição inicial, as geodésicas se comportam do mesmo jeito como no caso prévio da condição inicial ser tomada perto de $r_{H}$, e novamente encontramos atalhos para as branas dominadas por matéria e radiação antes que todas as geodésicas caiam no horizonte de eventos. Alguns resultados são mostrados na Fig. 7.2 .

\subsection{2 $M<0, Q^{2}=0, l^{2}>0$}

Como podemos ver da Fig. 7.1(b), para $M$ negativo e $l^{2}$ positivo e quando $k=-1$, $h$ descreve um buraco negro topológico com horizonte de eventos hiperbólico em um espaço assintoticamente AdS. Se $k \neq-1$, existe uma singularidade nua e a métrica é assintoticamente AdS.

As soluções para a equação de movimento da brana (7.5) apresentam as seguintes características. No caso de uma parede de domínio, quando $k=-1$, a brana cai no horizonte de eventos; enquanto que se $k \neq-1$, a solução para $\mathcal{R}(t)$ cresce.

Para $(\omega=0)$, a brana dominada por matéria cai na singularidade nua quando $k \neq-1$ e dentro do horizonte de eventos se $k=-1$. A brana dominada por radiação apresenta o mesmo comportamento.

Por outra parte, as soluções da Eq. (7.6) mostram que quando $k \neq-1$, as geodésicas crescem mais lentamente que a parede de domínio. Algumas delas são atalhos para as branas dominadas por matéria ou radiação, já que deixam as branas e retornam a elas antes de que as branas alcancem a singularidade nua. Além disso, se a velocidade inicial (negativa) da geodésica é grande o suficiente, esta pode cair 
na singularidade nua. No caso $k=-1$, todas as geodésicas caem no horizonte de eventos. Podemos ver alguns resultados na Fig.7.3.

7.3.3 $M>0, Q^{2}=0, l^{2}<0$

No caso de $M$ positivo, $l^{2}$ negativo e $k=1$, a métrica é de Sitter-Schwarzschild com horizontes de eventos e cosmológico dados pelos zeros de $h(\mathcal{R})$. Se $k \neq 1$, existe uma singularidade cosmológica em $\mathcal{R}=0$ no fundo assintoticamente de Sitter. Veja a Fig. $7.1(\mathrm{c})$

No caso de uma parede de domínio para $k=1$, as soluções para $\mathcal{R}(t)$ convergem ao horizonte de eventos ou ao horizonte cosmológico. Para $k \neq 1$, não existe uma solução já que $\mathcal{R}$ se torna uma coordenada tipo tempo. O mesmo comportamento é observado para branas dominadas por matéria e radiação.

Já que as soluções da equação de movimento da brana só aparecem quando $k=1$, as soluções da equação geodésica somente têm significado físico neste caso. Desta forma, achamos que as geodésicas alcançam o horizonte de eventos ou o horizonte cosmológico. No último caso, existem vários atalhos para as branas dominadas por matéria e radiação, assim como para a parede de domínio antes que todas elas alcancem o horizonte cosmológico. Alguns resultados são ilustrados na Fig .7.4.

\subsection{4 $M<0, Q^{2}=0, l^{2}<0$}

Quando $M$ e $l^{2}$ são ambos negativos, a métrica do bulk apresenta uma singularidade nua no espaço assintoticamente dS. Existe também um horizonte cosmológico cuja geometria é determinada por $k$, como podemos ver da Fig.7.1(d).

No caso de uma parede de domínio, para qualquer $k$, a solução $\mathcal{R}(t)$ converge ao horizonte cosmológico.

Quando $(\omega=0)$, a brana dominada por matéria ou cai na singularidade nua ou converge ao horizonte cosmológico independentemente do valor de $k$. Este mesmo resultado foi achado para uma brana dominada por radiação.

De outro lado, as geodésicas ou caem na singularidade nua quando a velocidade inicial é negativa o suficiente, ou convergem ao horizonte cosmológico para qualquer $k$. No último caso, achamos vários atalhos para a parede de domínio e as branas dominadas por matéria e radiação. Podemos ver alguns resultados na Fig. 7.5.

Agora consideremos as soluções carregadas. 
$R(\mathrm{t})$ para $\mathrm{M}>0, \mathrm{Q}^{2} \neq 0, \mathrm{k}=0, \mathrm{l}^{2}>0$

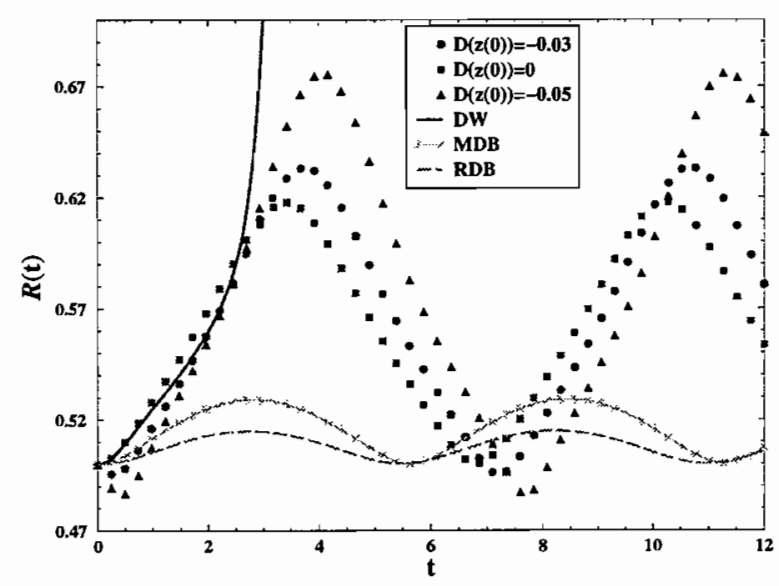

$R(\mathrm{t})$ para $\mathrm{M}>0, \mathrm{Q}^{2} \neq 0, \mathrm{k}=0, \mathrm{I}^{2}>0$

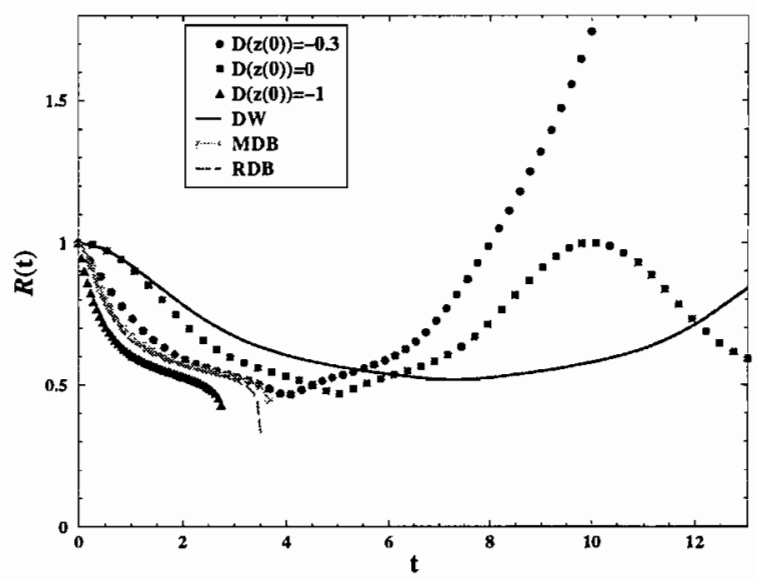

Figura 7.6: Evolução do fator de escala para uma parede de domínio e branas dominadas por matéria e radiação, e geodésicas quando $M>0, Q^{2} \neq 0$ e $l^{2}>0$.
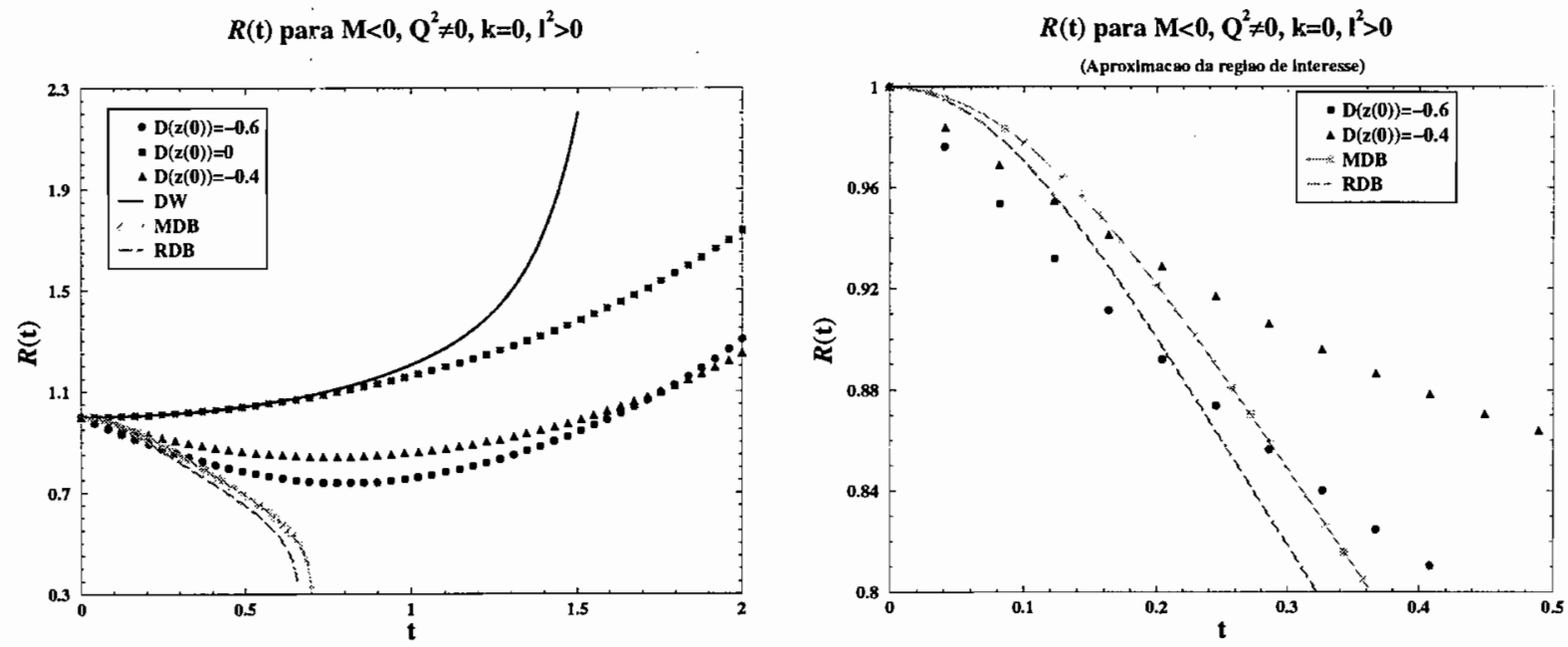

Figura 7.7: Evolução do fator de escala para uma parede de domínio e branas dominadas por matéria e radiação, e geodésicas quando $M<0, Q^{2} \neq 0$ e $l^{2}>0$.

\subsection{5 $M>0, Q^{2} \neq 0, l^{2}>0$}

Quando $M$ e $l^{2}$ são ambos positivos, para $k=-1$ a métrica descreve um buraco negro topológico carregado no espaço assintoticamente AdS com horizonte hiperbólico. Para $k \neq-1$, existe uma singularidade nua e o bulk é assintoticamente AdS. Veja a Fig. 

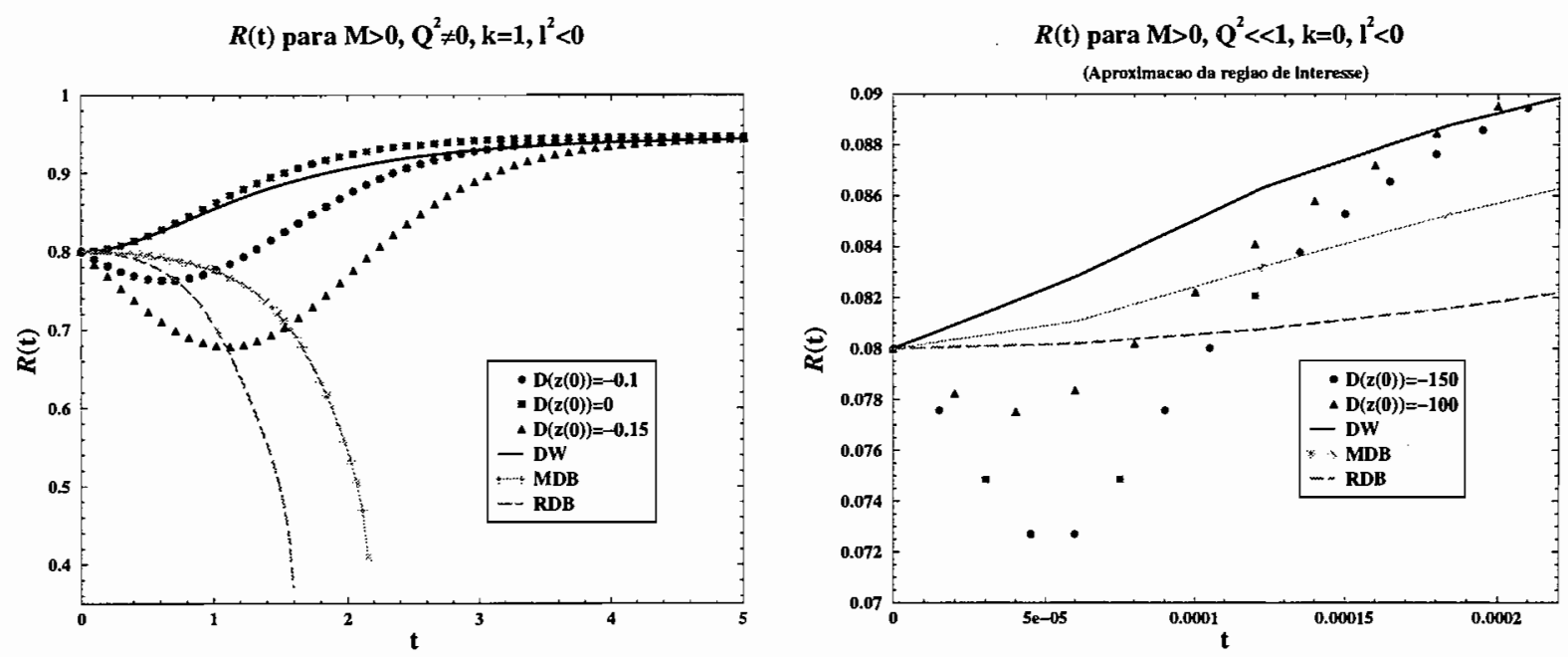

Figura 7.8: Evolução do fator de escala para uma parede de domínio e branas dominadas por matéria e radiação, e geodésicas quando $M>0, Q^{2} \neq 0$ e $l^{2}<0$.

$R(t)$ para $\mathrm{M}<0, \mathrm{Q}^{2} \neq 0, \mathrm{k}=1, \mathrm{I}^{2}<0$

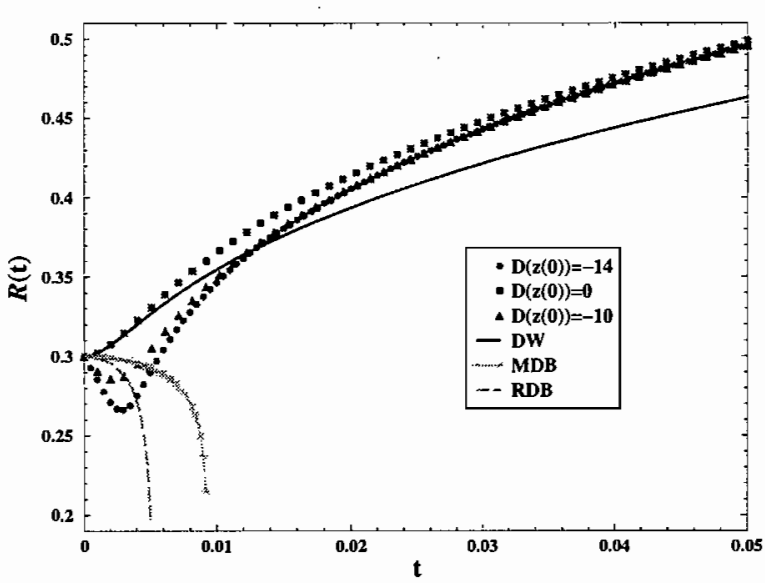

$R(\mathrm{t})$ para $\mathrm{M}<0, \mathrm{Q}^{2} \neq 0, \mathrm{k}=1, \mathrm{I}^{2}<0$

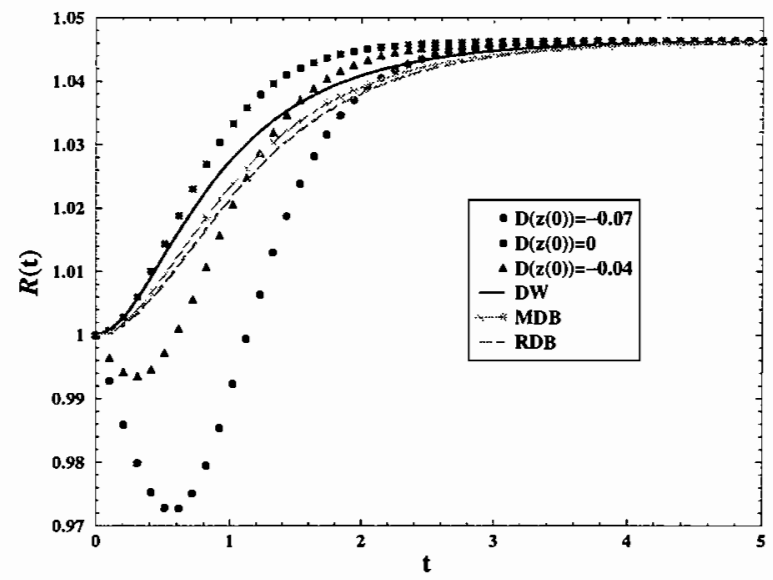

Figura 7.9: Evolução do fator de escala para uma parede de domínio e branas dominadas por matéria e radiação, e geodésicas quando $M<0, Q^{2} \neq 0$ e $l^{2}<0$.

7.1(e). Note que se a carga for pequena, todas as métricas descrevem buracos negros topológicos carregados em bulks AdS. Em particular, quando $k=1$, temos o bulk AdS-Reissner-Nordström.

Vejamos as soluções da equação de movimento da brana. No caso de uma parede de domínio, existem diferentes comportamentos para cada valor de $k$. Quando $k=0$, 
$\mathcal{R}(t)$ pula repetidas vezes para a condição inicial $\mathcal{R}(0)$ maior ou igual ao mínimo de $h(\mathcal{R})$, e diverge para o infinito para a escolha contrária. Quando $k=1, \mathcal{R}(t)$ diverge para o infinito. Quando $k=-1$, a parede de domínio cai no horizonte de eventos. Para cargas pequenas, $\mathcal{R}(t)$ converge ao horizonte de eventos exceto quando $k=1$, onde também pode crescer ou divergir ao infinito após certo valor inicial. No limite $Q \rightarrow 0$, também verificamos que nossas soluções convergem àquelas obtidas na seção 7.3.1.

Para uma brana dominada por matéria $(\omega=0)$, novamente achamos diferentes comportamentos para cada $k$. Quando $k=0, \mathcal{R}(t)$ pula repetidamente para a condição inicial $\mathcal{R}(0)$ menor ou igual ao mínimo de $h(\mathcal{R})$, e cai na singularidade nua para a escolha contrária. Quando $k=1, \mathcal{R}(t)$ vai para a singularidade nua. Quando $k=-1$, a brana cai no horizonte de eventos. Para pequenas cargas, a brana sempre cai dentro do horizonte de eventos. Novamente quando $Q \rightarrow 0$, recuperamos as soluções da seção 7.3.1.

No caso de uma brana dominada por radiação $(\omega=1 / 3)$, o comportamento da solução é, de alguma forma, similar ao caso da brana dominada por matéria. Quando $k=0, \mathcal{R}(t)$ pula repetidamente para a condição inicial $\mathcal{R}(0)$ menor ou igual ao mínimo de $h(\mathcal{R})$, mas cai na singularidade nua quando a condição inicial está perto de 0 ou quando é maior que o mínimo de $h(\mathcal{R})$. Quando $k=1, \mathcal{R}(t)$ vai para a singularidade nua. Quando $k=-1$, a brana cai no horizonte de eventos como no caso prévio. Para cargas pequenas, temos o mesmo comportamento que no caso da brana dominada por rnatéria.

Agora investigamos o comportamento para as geodésicas. Quando $k=0$, as geodésicas pulam repetidamente para a condição inicial menor ou igual ao mínimo de $h(\mathcal{R})$ da mesma forma que as branas dominadas por matéria ou radiação, produzindo muitos atalhos para todas as branas. No entanto, quando a condição inicial é maior que o mínimo de $h(\mathcal{R})$, as geodésicas podem continuar a pular ou escapar depois de uma primeira oscilação ou ainda cair na singularidade nua como resultado do aumento da velocidade inicial (negativa); neste caso, somente achamos atalhos para a parede de domínio sempre que as geodésicas completem no mínimo uma oscilação. Para cargas pequenas, um horizonte de eventos é formado e as geodésicas caem nele, mas existem ainda alguns atalhos pequenos para branas dominadas por matéria ou radiação. Quando $k=1$, as geodésicas crescem mais lentamente que a parede de domínio produzindo atalhos somente para as branas dominadas por matéria ou radiação. Para pequenas cargas, as geodésicas ou divergem ou caem no horizonte de eventos. Quando $k=-1$, todas as geodésicas caem dentro do horizonte de eventos como as branas e não existem atalhos. Para cargas pequenas, as geodésicas caem no horizonte de eventos mas podem produzir alguns atalhos logo no começo de suas trajetórias para as branas dominadas por matéria ou radiação. Alguns destes casos aparecem na Fig. 7.6. 
7.3.6 $M<0, Q^{2} \neq 0, l^{2}>0$

Para $M$ negativo, $l^{2}$ positivo e $k=-1$, a métrica descreve um buraco negro topológico carregado com horizonte hiperbólico no espaço-tempo assintoticamente AdS. Enquanto que para $k \neq-1$, existe uma singularidade nua e o bulk é assintoticamente AdS como é mostrado na Fig. 7.1(f).

No caso de $\omega=-1$ e para $k=-1$, a parede de domínio cai dentro do horizonte de eventos. Se, de outro lado, $k \neq-1$, a solução $\mathcal{R}(t)$ diverge ao infinito.

Quando consideramos a brana dominada por matéria para $k=-1$, a brana se comporta do mesmo jeito que a parede de domínio; porém, se $k \neq-1$, ela cai na singularidade nua. Este mesmo comportamento é observado para a brana dominada por radiação.

Quanto às soluções da equação geodésica, quando $k=-1$, as geodésicas caem no horizonte de eventos e algumas podem produzir atalhos para as branas dominadas por matéria ou radiação antes de sua queda. Se $k \neq-1$, as geodésicas crescem e produzem atalhos para as branas dominadas por matéria ou radiação. Alguns resultados são mostrados na Fig. 7.7.

\subsection{7 $M>0, Q^{2} \neq 0, l^{2}<0$}

No caso de $M$ positivo e $l^{2}$ negativo, a métrica descreve uma singularidade nua no espaço assintoticamente de Sitter com um horizonte cosmológico como vemos na Fig. 7.1(g). No entanto, para uma carga pequena, a métrica com $k=1$ se torna dSReissner-Nordström com horizontes de Cauchy, de eventos e cosmológico como pode ser visto na Fig. 7.1(h).

Para uma parede de domínio, a solução $\mathcal{R}(t)$ converge ao horizonte cosmológico para qualquer $k$. Para uma carga pequena, este comportamento também se aplica, exceto para $k=1$, quando a parede de domínio pode também cair no horizonte de eventos.

O caso de uma brana dominada por matéria apresenta um comportamento interessante. Quando $k=1, \mathcal{R}(t)$ pula quando a condição inicial $\mathcal{R}(0)$ é menor ou igual ao único ponto de sela em $h(\mathcal{R})$; de outra forma cai na singularidade nua ou converge ao horizonte cosmológico se a condição inicial $\mathcal{R}(0)$ está perto dele. Quando $k \neq 1$, as soluções convergem ao horizonte cosmológico. Para cargas pequenas, as soluções convergem ao horizonte cosmológico e também ao horizonte de eventos no caso $k=1$.

Quando consideramos a brana dominada por radiação, vemos que ou cai na singularidade nua ou converge ao horizonte cosmológico para qualquer $k$. Para uma pequena carga, as soluções se comportam do mesmo jeito, exceto se $k=1$ quando a brana converge para o horizonte cosmológico ou para o horizonte de eventos. 


\begin{tabular}{|c|c|c|c|c|c|c|c|c|c|c|c|c|}
\hline \hline & \multicolumn{1}{|c|}{ Parede de Dominio } & \multicolumn{3}{|c|}{ B. Dom. por Matéria } & \multicolumn{3}{|c|}{ B. Dom. por Radiação } \\
\hline Fig. & $t$ & $\tau$ & $\Delta \tau$ & $g / \gamma$ & $t$ & $\tau$ & $\Delta \tau$ & $g / \gamma$ & $t$ & $\tau$ & $\Delta \tau$ & $g / \gamma$ \\
\hline \hline 2 & - & - & - & - & .54 & .408 & .004 & 1.011 & .27 & .238 & .002 & 1.009 \\
\hline 3 & - & - & - & - & .23 & .25 & .01 & 1.047 & .18 & .191 & .005 & 1.033 \\
\hline 4 & 2.39 & .563 & .009 & 1.015 & 1.27 & .393 & .003 & 1.007 & .95 & .306 & .002 & 1.006 \\
\hline 4 & 3.51 & .76 & .03 & 1.035 & 2.33 & .67 & .02 & 1.024 & 1.74 & .56 & .01 & 1.019 \\
\hline 5 & 1.58 & .53 & .02 & 1.028 & .89 & .412 & .007 & 1.017 & .66 & .326 & .004 & 1.013 \\
\hline 5 & 2.20 & .69 & .04 & 1.061 & 1.45 & .63 & .03 & 1.046 & 1.06 & .54 & .02 & 1.039 \\
\hline 5 & - & - & - & - & .66 & .47 & .03 & 1.064 & .47 & .32 & .01 & 1.046 \\
\hline 5 & - & - & - & - & .44 & .300 & .006 & 1.021 & .33 & .224 & .003 & 1.015 \\
\hline 6 & 2.03 & .464 & .006 & 1.013 & .80 & .232 & .002 & 1.008 & .64 & .192 & .001 & 1.007 \\
\hline 6 & - & - & - & - & 1.11 & .318 & .007 & 1.022 & .94 & .283 & .006 & 1.020 \\
\hline 6 & 5.5 & 1.6 & .2 & 1.206 & - & - & - & - & - & - & - & - \\
\hline 6 & 6.2 & 2.5 & .1 & 1.074 & - & - & - & - & - & - & - & - \\
\hline 7 & - & - & - & - & .30 & .259 & .006 & 1.024 & .22 & .191 & .003 & 1.018 \\
\hline 7 & - & - & - & - & .175 & .169 & .002 & 1.011 & .13 & .126 & .001 & 1.008 \\
\hline 8 & 3.07 & .91 & .06 & 1.065 & 1.01 & .47 & .01 & 1.026 & .73 & .337 & .006 & 1.019 \\
\hline 8 & - & - & - & - & 1.56 & .76 & .07 & 1.094 & 1.1 & .51 & .03 & 1.061 \\
\hline 8 & $2.2 \mathrm{E}-4$ & $2 \mathrm{E}-3$ & $2 \mathrm{E}-4$ & 1.084 & $1.4 \mathrm{E}-4$ & $1.7 \mathrm{E}-3$ & $1 \mathrm{E}-4$ & 1.072 & $1.1 \mathrm{E}-4$ & $1.6 \mathrm{E}-3$ & $1 \mathrm{E}-4$ & 1.069 \\
\hline 8 & $1.9 \mathrm{E}-4$ & $1.8 \mathrm{E}-3$ & $7 \mathrm{E}-5$ & 1.035 & $1.1 \mathrm{E}-4$ & $1.4 \mathrm{E}-3$ & $3 \mathrm{E}-5$ & 1.024 & $8.2 \mathrm{E}-5$ & $1 . \mathrm{I} \mathrm{E}-3$ & $2 \mathrm{E}-5$ & 1.022 \\
\hline 9 & .013 & .036 & .004 & 1.106 & .0052 & .023 & .002 & 1.077 & .004 & .019 & .001 & 1.062 \\
\hline 9 & .012 & .035 & .002 & 1.056 & .0042 & .0186 & $5 \mathrm{E}-3$ & 1.030 & .0031 & .0139 & $3 \mathrm{E}-3$ & 1.023 \\
\hline 9 & 2.4 & .52 & .02 & 1.029 & 2.2 & .52 & .01 & 1.027 & 2.0 & .51 & .01 & 1.026 \\
\hline 9 & 1.5 & .355 & .004 & 1.010 & 1.3 & .339 & .003 & 1.008 & 1.1 & .314 & .002 & 1.007 \\
\hline \hline
\end{tabular}

Tabela 7.1: Tempo no bulk $t$, tempo na brana $\tau$, atrasos no tempo $\Delta \tau$ e razão entre os horizontes do gráviton e do fóton $g / \gamma$ para as geodésicas de atalho.

Vejamos o comportamento geodésico. Para qualquer $k$, todas as geodésicas convergem para o horizonte cosmológico e produzem vários atalhos para todos os casos de branas. Quando a carga é muito pequena, aparece um horizonte de eventos no caso $k=1 \mathrm{e}$ as geodésicas podem convergir ou para o horizonte cosmológico ou para o horizonte de eventos. As geodésicas que convergem ao horizonte cosmológico produzem atalhos para todos os casos de branas, enquanto que as geodésicas caindo no horizonte de eventos produzem atalhos somente para as branas dominadas por matéria ou radiação. De outro lado, quando $k \neq 1$, as geodésicas convergem ao horizonte cosmológico após produzirem alguns atalhos para todos os casos de branas, a menos que sua velocidade inicial (negativa) alcance um limiar após o qual elas caem na singularidade nua. Alguns resultados são ilustrados na Fig. 7.8. 
7.3.8 $M<0, Q^{2} \neq 0, l^{2}<0$

Quando $M$ e $l^{2}$ são ambos negativos, a métrica descreve uma singularidade nua no espaço assintoticamente dS para qualquer $k$. Veja a Fig. 7.1(i).

Para as soluções da equação de movimento da brana, no caso de uma parede de domínio, a solução converge ao horizonte cosmológico.

Quando consideramos uma brana dominada por matéria, $\mathcal{R}(t)$ cai na singularidade nua ou converge ao horizonte cosmológico para qualquer $k$. O mesmo comportamento aplica-se à brana dominada por radiação.

Quanto às geodésicas, elas convergem ao horizonte cosmológico e produzem vários atalhos para todos os casos de branas para qualquer $k$. No entanto, quando a condição inicial é tomada perto da singularidade e a velocidade inicial é negativa o suficiente, as geodésicas podem alcançar a singularidade e nenhum atalho aparece. Podemos ver alguns resultados na Fig. 7.9.

\subsection{Discussão dos Resultados}

Tio presente capítulo estudamos o comportamento de uma brana imersa em um espaço-tempo 6-dimensional de Sitter ou Anti de Sitter, contendo uma singularidade coberta por pelo menos um horizonte para as soluções tipo buraco negro, ou uma singularidade nua. O sistema de equações descrevendo este comportamento do ponto de vista de um observador no bulk é altamente não linear. Antes de resolver numericamente este sistema, consideramos flutuações ao redor de uma posição fixa da brana para ter um melhor compreensão do problema todo. Concluímos que o caso de uma parede de domínio com constante cosmológica efetiva nula em um bulk plano, reproduz a equação de movimento para um campo escalar como em 5 dimensões $[57,58]$. A posição de "equilíbrio" pode ser escolhida arbitrariamente, mas não existe estabilidade.

Resolvendo o sistema não linear completo, achamos diferentes comportamentos para os variados cenários aparecendo devido às combinações de $M, Q^{2}, k$ e $l^{2}$ levadas em conta. Escolhemos alguns valores típicos para $\omega$, i.e. paredes de domínio e branas dominadas por matéria e radiação, para ilustrar as soluções. Os resultados mostram branas escapando da singularidade, caindo dentro dela, convergindo a horizontes cosmológicos quando eles existem ou ainda pulando várias vezes entre um máximo e um mínimo.

O comportamento oscilatório achado em algumas soluções da equação de movimento da brana não é surpresa, já que em 5 dimensões um comportamento similar foi obtido em pesquisas recentes $[59,60,61]$, onde aparecem universos pulando de uma fase de contração a uma fase de expansão sem achar singularidades passadas 
ou futuras. Desta forma, estes resultados poderiam fornecer apoio à cosmologia sem singularidade ou aos, assim chamados, cenários de universos cíclicos [62].

Finalmente, estudamos também o comportamento geodésico em cada cenário achado neste capítulo. Contrariamente ao caso de brana estática visto no capítulo 5, onde os atalhos apareciam sob condições muito restritivas [34], o modelo presente de uma brana dinâmica imersa em um bulk estático apresenta atalhos em quase todos os casos e sob condições bem leves. Ainda mais, apesar do fato do atraso do tempo entre os tempos de vôo do gráviton e do fóton não ser percentualmente muito grande como em outros modelos [43] (o que é evidente da razão entre os horizontes do gráviton e do fóton), ele existe e pode eventualmente ser medido por um observador na brana, ainda que mais considerações são certamente necessárias em um modelo realista mais estrito. De outro lado, o fato dos atalhos serem abundantes nos cenários estudados empresta maior apoio à idéia de resolver o problema do horizonte por meio de termalização por intercâmbio de grávitons [43, 40], no entanto, devemos notar que isto não é uma prova da solução do problema ainda. 


\section{Capítulo 8}

\section{Conclusões}

Na presente tese de doutorado, consideramos 4 modelos em cosmologia de branas, a saber, o modelo de Binétruy, Deffayet e Langlois para uma brana estática em um bulk dinâmico [10, 47]; o modelo de Chamblin e Reall para paredes de domínio [56]; o modelo de brana estática em um bulk Anti de Sitter contendo uma singularidade protegida por pelo menos um horizonte [34]; e a generalização do modelo anterior para o caso de uma brana dinâmica em um bulk Anti de Sitter ou de Sitter, contendo uma singularidade que pode ser nua ou estar protegida [44].

No primeiro modelo considerado no capítalo 3 [33], vimos que tanto para a época de domínio da matéria como para aquela de domínio da radiação, o caminho mais curto está dentro da brana, sendo aquele seguido pelos fótons. O comportamento típico para as geodésicas gravitacionais neste modelo é de patamar, isto é, saindo da brana, elas tentam segui-la ficando quase paralelas a ela por um tempo indefinido. Assim, concluímos que a cosmologia deste modelo está inclusa na larga classe de cosmologias sem atalhos.

Nossa pesquisa então continuou considerando novos bulks contendo singularidades. Consideramos primeiramente um modelo de brana estática imersa em bulks tipo AdSSchwarzschild e AdS-Reissner-Nordström em 6 dimensões no capítulo 5 [34]. Derivamos a equação para a trajetória mais curta, que só depende da coordenada radial extra quando consideramos uma simetria na coordenada angular adicional, fato que também nos permitiu considerar seções espaciais curvas. Provamos que os atalhos só aparecem para um bulk de seção espacial positiva $(k=1)$, sempre que um conjunto de fortes restrições na energia e no tipo de matéria na brana, assim como a posição dela no bulk, sejam respeitadas. O ajuste fino na energia aparece já nas condições de junção para o caso AdS-Schwarzschild, enquanto que para o caso AdS-Reissner-Nordström, este ajuste vem da condição de positividade da carga e de existência de horizontes. Assim, os atalhos em bulks com singularidades protegidas aparecem sob as mesmas condições para buracos tipo Schwarzschild e Reissner-Nordström. O que significa que ambos os casos parecem ser equivalentes no estudo de atalhos. Com as restrições 
achadas, perde-se então a principal vantagem do caso AdS-Reissner-Nordström, isto é, a ausência de ajustes finos para a energia desde o início. A principal conseqüência deste modelo aponta para um aporte na solução do problema do horizonte [29, 30], já que as restrições para obter atalhos impõem também uma restrição no tamanho do universo, $z_{0} \approx l$, que corresponde a um universo primordial.

Continuando a procurar uma explicação alternativa ao problema do horizonte, estudamos a existência de atalhos no modelo de Chamblin e Reall para paredes de domínio [43], descrito no capítulo 6. Provamos que os atalhos existem sob condições bem leves e são abundantes, condição essencial para resolver o problema de homogeneidade. Certamente, se estes atalhos forem comuns antes da nucleosíntese em um modelo realista, eles poderiam fornecer uma alternativa à inflação para termalizar o universo primitivo, como é mencionado em [40]. No entanto, apesar do modelo de Chamblin e Reall não ser muito realista, ele fornece um primeiro estágio na compreensão dos atalhos em modelos com branas dinâmicas. Mostramos também no capítulo 6 que o atraso no tempo de vôo medido por um observador na brana, pode chegar a ser comparável com o tempo de vôo do próprio gráviton.

Motivados pela procura de um modelo mais realista, no capítulo 7 generalizamos o modelo de brana estática para um modelo de brana dinâmica, contendo matéria, imersa em um espaço-tempo de Sitter ou Anti de Sitter com singularidade nua ou coberta por pelo menos um horizonte [44]. Derivamos as equações de movimento para a brana do ponto de vista de um joservador no bulk e as resolvemos analiticamente na aproximação linear e numericamente para o sistema completo. No primeiro caso, foram consideradas flutuações na posição inicialmente fixa da braina e concluímos que, para uma parede de domínio em um bulk plano (modelo RS), a equação de movimento resultante é análoga àquela de um campo escalar livre como em 5 dimensões. A posição da brana pode se escolher arbitrariamente, mas não corresponde a um ponto estável. O sistema não linear completo foi resolvido em seguida, para os casos típicos de parede de domínio e branas dominadas por matéria e radiação, achando-se diversos comportamentos, a saber, branas caindo na singularidade, escapando dela, convergindo a horizontes cosmológicos ou pulando repetidamente entre um mínimo e um máximo. A novidade é precisamente este último comportamento, que também fora achado em 5 dimensões em pesquisas recentes [59, 60,61]. Desta maneira, nossos resultados oferecem apoio à cosmologia sem singularidades, onde os universos pulam de fases de contração a fases de expansão, ou à chamada cosmologia de universos cíclicos. Quanto às geodésicas, ao contrário do modelo de brana estática, onde os atalhos apareciam sob condições muito restritivas, neste modelo de brana dinâmica, eles aparecem em quase todos os casos considerados e sob condições bem naturais. Além do mais, o atraso entre os tempos de vôo do gráviton e do fóton não é percentualmente muito grande, mas existe e eventualmente pode ser medido pelo observador da brana. Assim, a abundância do atalhos nos modelos de branas dinâmicas emprestam maior apoio à idéia de resolver o problema de horizonte por meio de termalização por intercâmbio de grávitons. Porém, devemos notar que isto não é uma prova da solução 
do problema ainda.

Nossa conclusão final nos dá uma motivação para continuar explorando as implicações da Cosmologia de Branas para o nosso universo estudando outros modelos físicos que considerem as restrições dos resultados vindos das provas experimentais $[63,64,65,66]$, assim como a Teoria $M$ de Hor̆ava e Witten $[4,5]$. A conexão com o experimento é estabelecida através do espectro de potências das perturbações cosmológicas geradas durante a inflação [67]; em particular, devemos indicar que as perturbações tensoriais, que correspondem às ondas gravitacionais geradas nessa época, podem fornecer um meio para achar relíquias das dimensões extras na radiação cósmica de fundo.

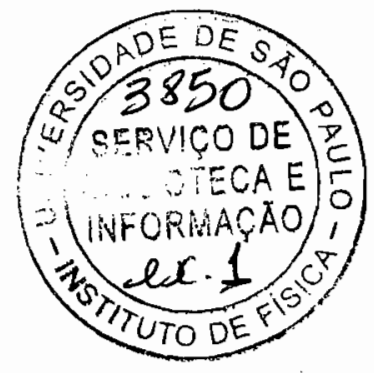




\section{Apêndice A}

\section{Geodésicas no Modelo BDL}

Vamos ilustrar a obtenção de geodésicas no modelo de Binétruy, Deffayet e Langlois para o caso de domínio da matéria $(\omega=0)$.

$>$ restart;

O fator de escala está dado pela Eq. (3.17),

$>$ ai : =unapply (simplify $(\operatorname{sqrt}(A(t)+B(t) * \cosh (m u * y)+C(t) * \sinh (m u * y))$.

$>$ sqrt, symbolic) *aOi(t),y,t);

$$
a i:=(y, t) \rightarrow \sqrt{\mathrm{A}(t)+\mathrm{B}(t) \cosh (\mu y)+\mathrm{C}(t) \sinh (\mu y)} \mathrm{a} 0 \mathrm{i}(t)
$$

onde $a_{0}$ é

$>\mathrm{a} 0 \mathrm{i}:=u n a p p l y\left(\mathrm{~F} *\left(\mathrm{H} * \mathrm{t}^{\wedge} 2+1 / 2 * \mathrm{t}\right)^{\wedge}(1 / 3), \mathrm{t}\right)$;

$$
a 0 i:=t \rightarrow F\left(H t^{2}+\frac{1}{2} t\right)^{1 / 3}
$$

lembrando que $\rho_{B}>0$. A função $n$ é calculada seguindo (3.8)

$>n i:=u n a p p l y(f a c t o r(\operatorname{diff}(\operatorname{ai}(y, t), t) /(\operatorname{diff}(a 0 i(t), t))), y, t)$;

$$
\begin{aligned}
n i:= & (y, t) \rightarrow \frac{1}{2}\left(6 \operatorname{diff}(\mathrm{A}(t), t) H t^{2}+3 \operatorname{diff}(\mathrm{A}(t), t) t\right. \\
& +6 \operatorname{diff}(\mathrm{B}(t), t) \cosh (\mu y) H t^{2}+3 \operatorname{diff}(\mathrm{B}(t), t) \cosh (\mu y) t \\
& +6 \operatorname{diff}(\mathrm{C}(t), t) \sinh (\mu y) H t^{2}+3 \operatorname{diff}(\mathrm{C}(t), t) \sinh (\mu y) t \\
& +8 H t \mathrm{~A}(t)+8 H t \mathrm{~B}(t) \cosh (\mu y)+8 H t \mathrm{C}(t) \sinh (\mu y) \\
& +2 \mathrm{~A}(t)+2 \mathrm{~B}(t) \cosh (\mu y)+2 \mathrm{C}(t) \sinh (\mu y)) /( \\
& \sqrt{\mathrm{A}(t)+\mathrm{B}(t) \cosh (\mu y)+\mathrm{C}(t) \sinh (\mu y)}(4 H t+1))
\end{aligned}
$$

A densidade de energia ordinária em cosmologia é dada por

$>$ rhoo: $=r h o_{-} s *\left(a 0 i(t) / a_{-} s\right)^{\wedge}(-3)$;

$$
\rho 0:=\frac{r h o_{-} s a_{-} s^{3}}{F^{3}\left(H t^{2}+\frac{1}{2} t\right)}
$$


No que segue, fazemos uma lista de todos os parâmetros que aparecerão no cálculo. $\mu$ está dado por (3.7), $\rho_{\Lambda}$ (aqui chamado de rho_l) pode ser obtido de (3.12), $\rho_{B}$ segue de (3.14) $\operatorname{com} \lambda=0$, e $\kappa$ corresponde à equação (3.19).

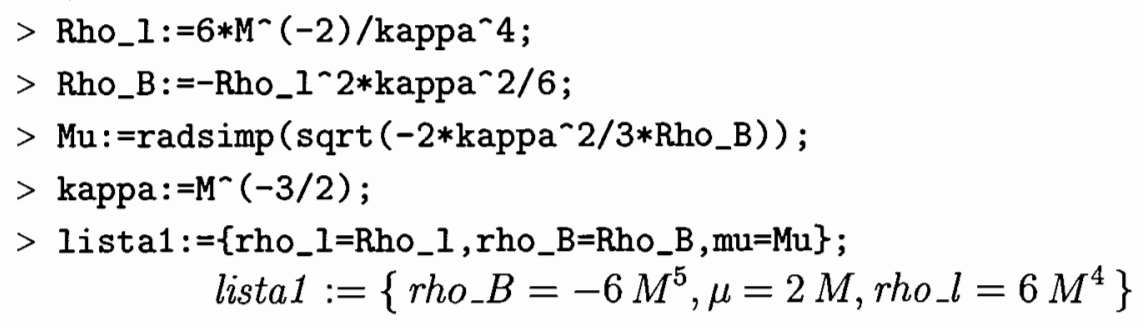

Os coeficientes $A, B$ e $C$ estão dados por

$>A 1:=$ radsimp (subs $\left(\right.$ lista $1,1 / 2 *\left(1+\right.$ kappa $^{*} 2 *($ rho_l $1+$ rho $) \sim 2 /(6 *$ rho_B $\left.\left.)\right)\right)$ );

$$
A 1:=-\frac{1}{72} \frac{\rho\left(12 M^{4}+\rho\right)}{M^{8}}
$$

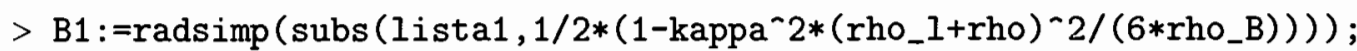

$$
B 1:=\frac{1}{72} \frac{72 M^{8}+12 M^{4} \rho+\rho^{2}}{M^{8}}
$$

> C1: :radsimp(subs (lista1,-kappa*(rho_l+rho)/sqrt(-6*rho_B)));

$$
C 1:=\frac{1}{6} \frac{-6 M^{4}-\rho}{M^{4}}
$$

Também definimos a constante $H$ aparecendo em rho_0

$>\mathrm{H}:=1 / 8 * \mathrm{kappa}^{\wedge} 2 * \mathrm{Rho}_{-} \mathrm{l}$;

Assim, $\rho$ fica como

$$
H:=\frac{3}{4} M
$$

$>$ rho1:=subs (lista1, rho0);

$$
\rho 1:=\frac{r h o_{\_} s a_{\_} s^{3}}{F^{3}\left(\frac{3}{4} M t^{2}+\frac{1}{2} t\right)}
$$

e $A, B$ e $C$ serão

$>$ Anew: $=\operatorname{convert}\left(\operatorname{subs}\left(F=a_{-} s *\left(k a p p a-2 * r h o \_s\right)-(1 / 3)\right.\right.$, subs (lista1,

$>\operatorname{subs}($ rho=rho1, A1))), parfrac, $t)$;

$$
\text { Anew }:=-\frac{1}{6} \frac{1}{M t}-\frac{1}{18} \frac{1}{M^{2} t^{2}}+\frac{1}{2} \frac{1}{3 M t+2}-\frac{1}{2} \frac{1}{(3 M t+2)^{2}}
$$

No limite de $t$ grande (domínio da matéria), $A$ é praticamente zero,

$>$ Bnew: $=$ convert $\left(\operatorname{subs}\left(F=a_{-} s *(k a p p a-2 * \text { rho_s })^{-}(1 / 3), \operatorname{subs}(\right.\right.$ lista1, subs (

$>$ rho=rho1, B1))), parfrac, t);

$$
B \text { new }:=1+\frac{1}{6} \frac{1}{M t}+\frac{1}{18} \frac{1}{M^{2} t^{2}}-\frac{1}{2} \frac{1}{3 M t+2}+\frac{1}{2} \frac{1}{(3 M t+2)^{2}}
$$

$B$ é praticamente 1 , 
$>$ Cnew:=convert (subs (F=a_s*(kappa`2*rho_s) ${ }^{-}(1 / 3)$, subs (lista1, subs (

$>$ rho=rho1, C1))), parfrac,t);

e $C$ é -1 . Portanto,

$$
\text { Cnew }:=-1-\frac{1}{3} \frac{1}{M t}+\frac{1}{3 M t+2}
$$

$$
\begin{aligned}
>\text { lista2 }:=\{\mathrm{at}=0, \mathrm{~b}=1, \mathrm{c}=-1\} & ; \\
& \text { lista2 }:=\{c=-1, b=1, a t=0\}
\end{aligned}
$$

O termo dominante em $a_{0}$ é o termo quadrático em $t$, assim finalmente teremos para $a_{0}, a$ e $n$

$$
\begin{gathered}
>\text { a0: }=\operatorname{unapply}\left(\operatorname{subs}\left(\text { lista2 }, \operatorname{subs}\left(\{\mathrm{A}(\mathrm{t})=\mathrm{at}, \mathrm{B}(\mathrm{t})=\mathrm{b}, \mathrm{C}(\mathrm{t})=\mathrm{c}\}, \mathrm{F} *\left(\mathrm{H} * \mathrm{t}^{\wedge} 2\right)^{\wedge}(1 / 3)\right)\right), \mathrm{t}\right) ; \\
\qquad a 0:=t \rightarrow \frac{1}{4} F 3^{1 / 3} 4^{2 / 3}\left(M t^{2}\right)^{1 / 3} \\
>\mathrm{a}:=\operatorname{unapply}(\operatorname{subs}(\operatorname{lista2}, \operatorname{subs}(\{\mathrm{A}(\mathrm{t})=\mathrm{at}, \mathrm{B}(\mathrm{t})=\mathrm{b}, \mathrm{C}(\mathrm{t})=\mathrm{c}\}, \operatorname{sqrt}(\mathrm{A}(\mathrm{t})+\mathrm{B}(\mathrm{t}) * \\
>\cosh (\operatorname{mu} * \mathrm{y})+\mathrm{C}(\mathrm{t}) * \sinh (\operatorname{mu} * \mathrm{y})) * \mathrm{a} 0(\mathrm{t}))), \mathrm{y}, \mathrm{t}) ; \\
a:=(y, t) \rightarrow \frac{1}{4} \sqrt{\cosh (\mu y)-\sinh (\mu y)} F 3^{1 / 3} 4^{2 / 3}\left(M t^{2}\right)^{1 / 3} \\
>\mathrm{n}:=\text { unapply(diff }(\mathrm{a}(\mathrm{y}, \mathrm{t}), \mathrm{t}) /(\operatorname{diff}(\mathrm{a} 0(\mathrm{t}), \mathrm{t})), \mathrm{y}, \mathrm{t}) ; \\
n:=(y, t) \rightarrow \sqrt{\cosh (\mu y)-\sinh (\mu y)}
\end{gathered}
$$

Quebramos a equação de Euler-Lagrange em 5 partes. Aqui estamos chamando $w 1=\ddot{y}$ and $x 1=\dot{y}$.

$$
\begin{aligned}
& >o p 1:=-\operatorname{subs}(x 1(t)=x 1, \operatorname{subs}(\operatorname{diff}(x 1(t), t)=w 1, \operatorname{subs}(\operatorname{diff}(y(t), t)=x 1(t), \\
& >\operatorname{diff}(y(t), t \$ 2)))) ; \\
& \quad o p 1:=-w 1 \\
& >\operatorname{op} 2:=\operatorname{collect}(\operatorname{simplify}(\operatorname{expand}(\operatorname{subs}(\mathrm{x} 1(\mathrm{t})=\mathrm{x} 1, \operatorname{subs}(\operatorname{diff}(\mathrm{x} 1(\mathrm{t}), \mathrm{t})=\mathrm{w} 1, \\
& >\operatorname{subs}(\operatorname{diff}(\mathrm{y}(\mathrm{t}), \mathrm{t})=\mathrm{x} 1(\mathrm{t}),(\operatorname{expand}(\operatorname{simplify}(\operatorname{diff}(\mathrm{a}(\mathrm{y}(\mathrm{t}), \mathrm{t}), \mathrm{t}) / \mathrm{a}(\mathrm{y}(\mathrm{t}), \mathrm{t})) \\
& >+\operatorname{simplify}(\operatorname{diff}(\mathrm{n}(\mathrm{y}(\mathrm{t}), \mathrm{t}), \mathrm{t}) / \mathrm{n}(\mathrm{y}(\mathrm{t}), \mathrm{t}))) * \operatorname{diff}(\mathrm{y}(\mathrm{t}), \mathrm{t}))))), \mathrm{x} 1) ; \\
& \text { op2 }:=-\mu x 1^{2}+\frac{2}{3} \frac{x 1}{t}
\end{aligned}
$$

$$
\begin{aligned}
& >\text { op3:=simplify ( } \operatorname{subs}(x 1(t)=x 1, \operatorname{subs}(\operatorname{diff}(x 1(t), t)=w 1, \operatorname{subs}(\operatorname{diff}(y(t), t) \\
& >=x 1(t),(2 * \operatorname{subs}(\operatorname{dot}=\operatorname{diff}(y(t), t), \operatorname{subs}(y=y(t), \operatorname{diff}(\operatorname{subs}(y(t)=y, \operatorname{subs}( \\
& >\operatorname{diff}(y(t), t)=\operatorname{dot}, n(y, t))), y) / n(y, t))) \quad-\operatorname{subs}(y=y(t), \operatorname{diff}(a(y, t), y) / \\
& >a(y, t))) *(\operatorname{diff}(y(t), t)) \sim 2)))) \text {; } \\
& \text { op } 3:=-\frac{1}{2} \mu x 1^{2}
\end{aligned}
$$

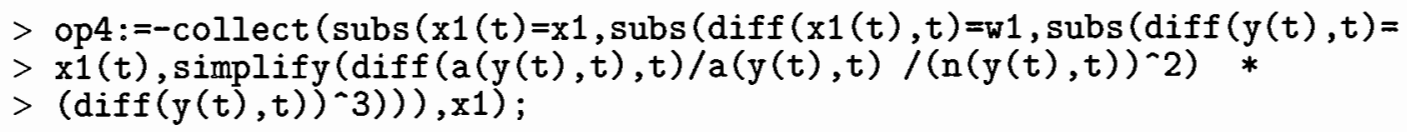




$$
\begin{aligned}
\text { op } 4 & :=\frac{1}{2} \frac{\mu x 1^{4}}{\cosh (\mu \mathrm{y}(t))-\sinh (\mu \mathrm{y}(t))} \\
& -\frac{2}{3} \frac{x 1^{3}}{t(\cosh (\mu \mathrm{y}(t))-\sinh (\mu \mathrm{y}(t)))}
\end{aligned}
$$

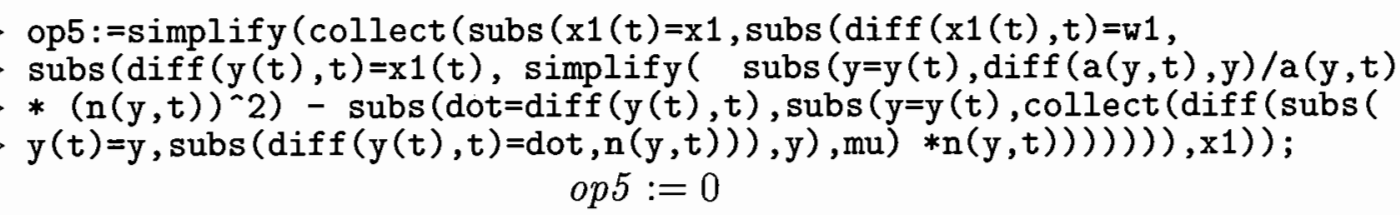

Fazemos $z=\mu y$ e $t=t_{0} x$ com $t_{0}=2.83824 \times 10^{(17)}$, a idade do universo.

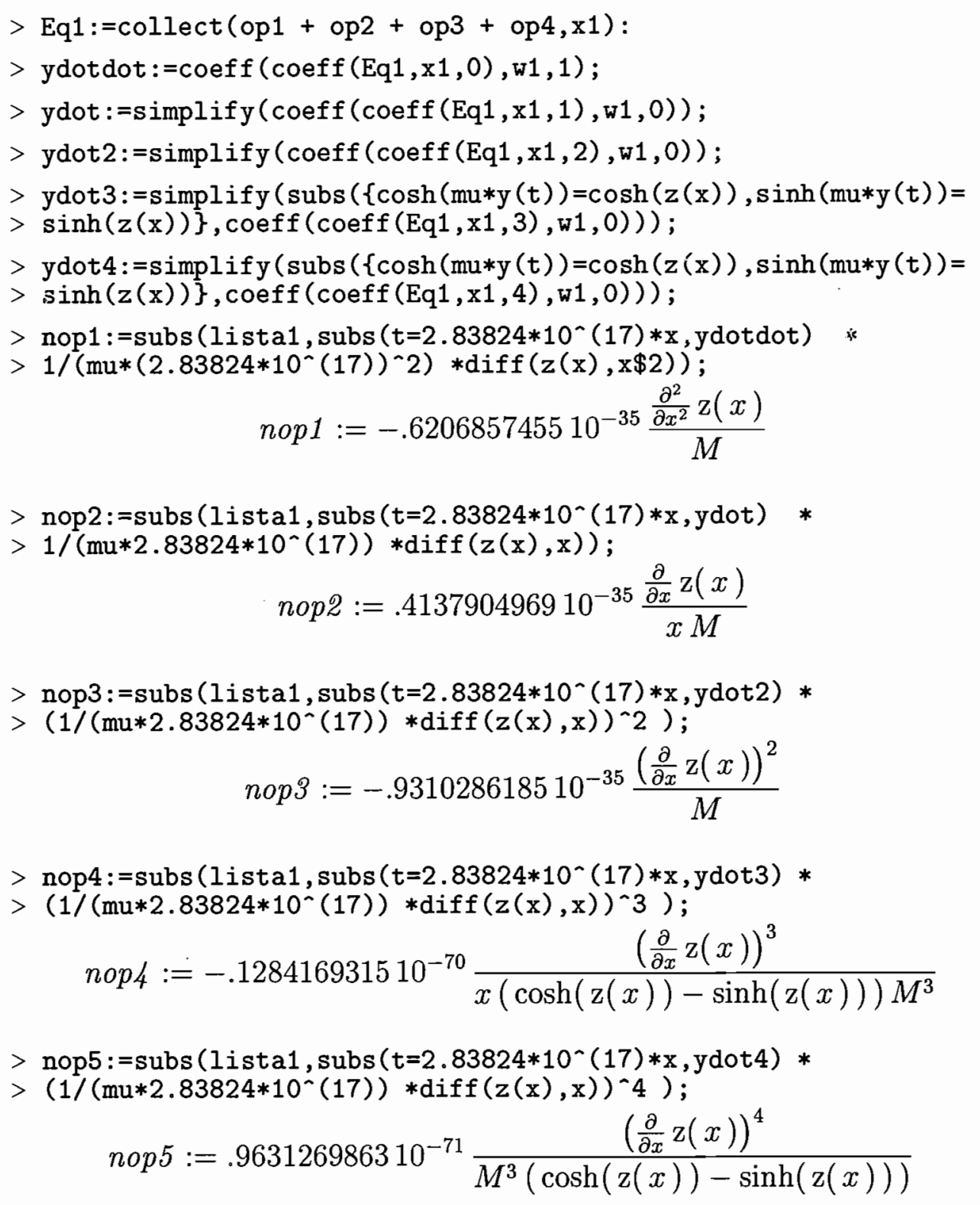


Já que os 3 primeiros termos são dominantes frente aos 2 últimos, nossa equação terá finalmente a forma

$>$ Equation:=evalf ( (nop1+nop2+nop3));

$>$ numer(Equation);

$$
\text { Equation }:=-6\left(\frac{\partial^{2}}{\partial x^{2}} \mathrm{z}(x)\right) x+4\left(\frac{\partial}{\partial x} \mathrm{z}(x)\right)-9\left(\frac{\partial}{\partial x} \mathrm{z}(x)\right)^{2} x
$$

\section{Solução Analítica}

$>$ dsolve(\{Equation2\}, $z(x))$;

$$
\begin{aligned}
\mathrm{z}(x) & =\frac{2}{9} \ln \left(729 x^{5}+1000_{-} C 1^{3}\right)+\frac{4}{9} \ln \left(9 x^{5 / 3}+10_{-} C 1\right) \\
& -\frac{2}{9} \ln \left(81 x^{10 / 3}-90 x^{5 / 3}{ }_{-} C 1+100_{-} C 1^{2}\right)+\ldots C 2
\end{aligned}
$$

Escolhendo cuidadosamente as condições iniciais podemos evitar a aparição de termos imaginários na solução. Vejamos por exemplo como foi obtida uma das curvas da Fig.(3.3)

$$
\begin{aligned}
& >\text { dsolve }(\{\text { Equation }, z(1)=0, D(z)(1)=10 *(8)\}, z(x)) \\
& >\text { plot }(\operatorname{Re}(\text { op }(2, ")), x=0.9 .2) ; \\
& z(x)=\frac{2}{9} \ln \left(729 x^{5}-\frac{728999975700000269999999}{1000000000000000000000}\right) \\
& +\frac{4}{9} \ln \left(9 x^{5 / 3}-\frac{89999999}{10000000}\right) \\
& -\frac{2}{9} \ln \left(81 x^{10 / 3}+\frac{809999991}{10000000} x^{5 / 3}+\frac{8099999820000001}{100000000000000}\right) \\
& -\frac{2}{9} \ln \left(\frac{24299999730000001}{1000000000000000000000}\right)+\frac{4}{9} \ln (10000000) \\
& +\frac{2}{9} \ln \left(\frac{24299999730000001}{100000000000000}\right)
\end{aligned}
$$

\section{Solução Numérica}

$>$ ff: $=$ dsolve $\left(\left\{\right.\right.$ Equation $\left., z(1)=0, D(z)(1)=10^{-}(8)\right\}, z(x)$, type=numeric,

$>$ method=rkf 45, 'output' $=$ 'procedurelist') ;

ff $:=\operatorname{proc}\left(\operatorname{rkf} 45_{-} x\right) \ldots$ end

$>$ with(plots):

$>\operatorname{odeplot}(f f,[x, z(x)], 1 \ldots 2)$;

E vemos que ambas são compatíveis. 


\section{Apêndice B}

\section{Atalhos no bulk AdS-Reissner-Nordström}

Vamos ilustrar a obtenção dos atalhos mostrados no capítulo 4 para o caso de um bulk AdS-Reissner-Nordström em 6 dimensões.

$>$ restart;

A função $h$ está definida em (4.17)como

$>\mathrm{h}:=$ unapply $\left(1+\mathrm{z}^{\wedge} 2 / \mathrm{I}^{\wedge} 2-\mathrm{M} / \mathrm{z}^{\wedge} 3+\mathrm{Q}^{\wedge} 2 / \mathrm{z}^{\vee} 6, \mathrm{z}\right)$;

$$
h:=z \rightarrow 1+\frac{z^{2}}{l^{2}}-\frac{M}{z^{3}}+\frac{Q^{2}}{z^{6}}
$$

Definimos também a derivada de $h$

$>\mathrm{dh}:=u n a p p l y(\operatorname{diff}(\mathrm{h}(\mathrm{z}), \mathrm{z}), \mathrm{z})$;

$$
d h:=z \rightarrow 2 \frac{z}{l^{2}}+3 \frac{M}{z^{4}}-6 \frac{Q^{2}}{z^{7}}
$$

$M$ e $Q$ podem ser escritos em termos da energia $\varepsilon$

$>\mathrm{M}:=\left(2+8 / 3 * \mathrm{z}^{\wedge} 2 / \mathrm{I}^{\wedge} 2+8 / 3 *\right.$ omega $* \mathrm{z} 0^{\wedge} 2 *$ epsilon` 2$) * \mathrm{z} 0^{\wedge} 3$;

$$
M:=\left(2+\frac{8}{3} \frac{z 0^{2}}{l^{2}}+\frac{8}{3} \omega z 0^{2} \varepsilon^{2}\right) z 0^{3}
$$

$>Q:=\operatorname{sqrt}\left(\left(1+5 / 3 * z 0^{\wedge} 2 / 1 \wedge 2+8 / 3 *\right.\right.$ omega*z0^ $2 *$ epsilon`2 +

$>z 0^{\wedge} 2 *$ epsilon 2$\left.) * z 0^{\wedge} 6\right)$;

$$
Q:=\sqrt{\left(1+\frac{5}{3} \frac{z 0^{2}}{l^{2}}+\frac{8}{3} \omega z 0^{2} \varepsilon^{2}+z 0^{2} \varepsilon^{2}\right) z 0^{6}}
$$

Escolhemos agora todos os parâmetros de acordo com a discussão no capítulo 4. Para $\omega$ temos

$>1:=1 ;$ omega: $=-9 / 10 ;$

$$
\begin{gathered}
l:=1 \\
\omega:=\frac{-9}{10}
\end{gathered}
$$


A posição da brana $z_{0}$ deve ser menor que

Escolhemos

$>1 / 2 * \operatorname{sqrt}(-1 *(4 *$ omega +3$) /(1+$ omega $))$;

$>z 0:=1$;

$$
\frac{1}{2} \sqrt{6}
$$

A energia $\varepsilon$ deve estar entre

$$
z 0:=1
$$

$>$ e $1:=1 /(32 *$ omega` 2$) *(-32 *$ omega*z0^-2/1^2+9+3*sqrt (-64*omega*

$>\mathrm{z} 0^{\wedge} 2 / 1 \wedge 2+9-64 * z 0^{\wedge} 2 / 1 \wedge 2 *$ omega^ 2$\left.)\right)$;

$>\mathrm{e} 2:=1 /(8 *$ omega +3$) *\left(-3-5 * \mathrm{z}^{-}-2 / 1 \wedge 2\right)$;

$$
\begin{gathered}
e 1:=\frac{35}{24}+\frac{5}{72} \sqrt{41} \\
e \mathscr{2}:=\frac{40}{21}
\end{gathered}
$$

$>\operatorname{evalf}(e 1)$; evalf (e2);

\subsection{8}

\subsection{5}

Escolhemos

$>$ epsilon: $=\operatorname{sqrt}(238 / 125)$;

Plotamos $h(z)$

$$
\varepsilon:=\frac{1}{25} \cdot \sqrt{1190}
$$

$>\operatorname{plot}(\mathrm{h}(\mathrm{z}), z=0.2 \ldots 1$, thickness $=2$, labelfont=[TIMES, BOLD , 28] ,

$>$ axesfont $=$ [TIMES, BOLD, 18]);

A equação geodésica vem dada por (4.30)

$$
\begin{aligned}
& >\text { geo: }=\operatorname{diff}(z(t), t \$ 2)+(\operatorname{diff}(z(t), t))^{-2} 2 *(1 / z(t)-(3 * \operatorname{dh}(z(t))) / \\
& >(2 * h(z(t))))+h(z(t)) *((\operatorname{dh}(z(t))) /(2)-(\mathrm{h}(\mathrm{z}(\mathrm{t}))) /(\mathrm{z}(\mathrm{t})))=0 ; \\
& \text { geo }:=\left(\frac{\partial^{2}}{\partial t^{2}} \mathrm{z}(t)\right) \\
& \quad+\left(\frac{\partial}{\partial t} \mathrm{z}(t)\right)^{2}\left(\frac{1}{\mathrm{z}(t)}-\frac{3}{2} \frac{2 \mathrm{z}(t)+\frac{182}{625} \frac{1}{\mathrm{z}(t)^{4}}-\frac{4}{625} \frac{1}{\mathrm{z}(t)^{7}}}{1+\mathrm{z}(t)^{2}-\frac{182}{1875} \frac{1}{\mathrm{z}(t)^{3}}+\frac{2}{1875} \frac{1}{\mathrm{z}(t)^{6}}}\right)+ \\
& \left(1+\mathrm{z}(t)^{2}-\frac{182}{1875} \frac{1}{\mathrm{z}(t)^{3}}+\frac{2}{1875} \frac{1}{\mathrm{z}(t)^{6}}\right)\left(\mathrm{z}(t)+\frac{91}{625} \frac{1}{\mathrm{z}(t)^{4}}\right. \\
& \left.\quad-\frac{2}{625} \frac{1}{\mathrm{z}(t)^{7}}-\frac{1}{\mathrm{z}(t)^{2}-\frac{182}{1875} \frac{1}{\mathrm{z}(t)^{3}}+\frac{2}{1875} \frac{1}{\mathrm{z}(t)^{6}}}\right)=0
\end{aligned}
$$

Resolvemos esta equação numericamente: 
$>$ sol0: $=$ dsolve $(\{$ geo $, z(0)=z 0, D(z)(0)=-0.5\}, z(t)$, type=numeric, $>$ 'output' $=$ 'procedurelist');

solo $:=\operatorname{proc}\left(\operatorname{rkf} 45_{-} x\right) \ldots$ end

E plotamos os resultados para várias velocidades iniciais.

$>$ with (plots): graph0:=odeplot (solo, $[t, z(t)], 0 . .0 .8$, style=POINT,

$>$ symbol=CROSS):

$>$ sol1: $=$ dsolve $(\{$ geo, $z(0)=z 0, D(z)(0)=-0.7\}, z(t)$, type=numeric,

$>$ 'output'='procedurelist'); with(plots): graph1:=odeplot (sol1,

$>[t, z(t)], 0.1 .1$, style=POINT, symbol=BOX, color $=$ RED $):$

sol1 := proc(rkf45_x $\ldots$ end

$>$ sol2: $=$ dsolve $(\{g e o, z(0)=z 0, D(z)(0)=-0.9\}, z(t)$, type=numeric,

$>$ 'output'='procedurelist'); with(plots): graph2:=odeplot (sol2,

$>[t, z(t)], 0.1 .6$, style=POINT, symbol=CIRCLE, color=BLUE):

sol2 := proc(rkf45_x $\ldots$ end

$>$ sol3: $=$ dsolve $(\{$ geo, $z(0)=z 0, D(z)(0)=-1.2\}, z(t)$, type=numeric,

$>$ 'output'='procedurelist'); with(plots): graph3:=odeplot (sol3,

$>[t, z(t)], 0.5$, style=POINT, symbol=DIAMOND, color=MAGENTA):

sol3 $:=\operatorname{proc}\left(\operatorname{rkf} 45_{-} x\right) \ldots$ end

$>$ plot (\{graph0,graph1, graph2, graph3\}, view=[0..5,0.4 ..1.1],

$>$ labelfont $=[$ TIMES, BOLD ,28], axesfont $=[$ TIMES, BOLD ,18]) ; 


\section{Apêndice C}

\section{Solução das Equações no Modelo de Chamblin e Reall}

Vamos ilustrar a obtenção de geodésicas de (6.7) e da dinâmica da parede de domínio a partir de (6.6), no caso das soluções tipo-III.

$>$ restart;

Definimos os parâmetros do problema:

$>\operatorname{gam}:=\operatorname{sqrt}\left((\mathrm{d}-3) /\left(2 * \mathrm{k} * \operatorname{Lambda} *\left(1-\mathrm{b}^{\wedge} 2\right)\right)\right)$;

$$
\text { gam }:=\frac{1}{2} \sqrt{2} \sqrt{\frac{d-3}{k \Lambda\left(1-b^{2}\right)}}
$$

$>\mathrm{b}:=1 / 2 *$ beta*sqrt $(\mathrm{d}-2)$;

$$
b:=\frac{1}{2} \beta \sqrt{d-2}
$$

$>$ Lambda: $=\mathrm{V} 0 * \exp (2 * \mathrm{~b} * \mathrm{phi} .0) /(\mathrm{d}-2) ;$

$$
\Lambda:=\frac{V 0 \mathrm{e}^{(\beta \sqrt{d-2} \phi 0)}}{d-2}
$$

$>$ alpha: $=2 /(\operatorname{beta} *(d-2))$;

O fator de escala

$$
\alpha:=2 \frac{1}{\beta(d-2)}
$$

$>\mathrm{R}:=$ unapply $\left(\operatorname{gam} * \mathrm{r}^{\wedge}\left(\mathrm{b}^{\wedge} 2 /\left(1+\mathrm{b}^{\wedge} 2\right)\right), \mathrm{r}\right)$;

$$
R:=r \rightarrow \frac{1}{2} \sqrt{2} \sqrt{\frac{(d-3)(d-2)}{k V 0 \mathrm{e}^{(\beta \sqrt{d-2} \phi 0)}\left(1-\frac{1}{4} \beta^{2}(d-2)\right)}} r\left(1 / 4 \frac{\beta^{2}(d-2)}{1+1 / 4 \beta^{2}(d-2)}\right)
$$

e o potencial $F$ 
$>\mathrm{F}:=$ unapply $\left(-(\mathrm{d}-3) * \mathrm{~b}^{\wedge} 4 /\left(2 * \mathrm{k} *\left(1-\mathrm{b}^{\wedge} 2\right) *\left(1+\mathrm{b}^{\wedge} 2 *(\mathrm{~d}-3)\right)\right)-\right.$

$>\mathrm{M} * \operatorname{gam}^{\sim} 2 * \mathrm{~b}^{\wedge} 4 *(\mathrm{R}(\mathrm{r}) / \mathrm{gam})-\left(-\left(\mathrm{d}-3+1 / \mathrm{b}^{\wedge} 2\right)\right)-\mathrm{V} 1^{\wedge} 2 * \mathrm{ex}$

$\left.>\mathrm{p}(2 * \text { phi.0/b)*gam^2/(8*(d-2)^2)*(R(r)/gam })^{-}\left(-\left(2 *\left(1 / \mathrm{b}^{\wedge} 2-1\right)\right)\right), \mathrm{r}\right)$;

$$
\begin{aligned}
F:=r & \rightarrow-\frac{1}{32} \frac{(d-3) \beta^{4}(d-2)^{2}}{k\left(1-\frac{1}{4} \beta^{2}(d-2)\right)\left(1+\frac{1}{4} \beta^{2}(d-2)(d-3)\right)} \\
& -\frac{1}{32} \frac{M(d-3)(d-2)^{3} \beta^{4}\left(r\left(1 / 4 \frac{\beta^{2}(d-2)}{1+1 / 4 \beta^{2}(d-2)}\right)\right)^{\left(-d+3-4 \frac{1}{\beta^{2}(d-2)}\right)}}{k V 0 \mathrm{e}^{(\beta \sqrt{d-2} \phi 0)}\left(1-\frac{1}{4} \beta^{2}(d-2)\right)} \\
& -\frac{1}{16} \frac{V 1^{2} \mathrm{e}^{\left(4 \frac{\phi 0}{\beta \sqrt{d-2}}\right)}(d-3)\left(r\left(1 / 4 \frac{\beta^{2}(d-2)}{1+1 / 4 \beta^{2}(d-2)}\right)\right)^{\left(-8 \frac{1}{\beta^{2}(d-2)}+2\right)}}{k V 0 \mathrm{e}^{(\beta \sqrt{d-2} \phi 0)}(d-2)\left(1-\frac{1}{4} \beta^{2}(d-2)\right)}
\end{aligned}
$$

O coeficiente $U$ na métrica está dado por

$>\mathrm{U}:=$ unapply $\left(\left(1+\mathrm{b}^{\wedge} 2\right)^{\wedge} 2 * \mathrm{r}^{\wedge}\left(2 /\left(1+\mathrm{b}^{\wedge} 2\right)\right) *\left(-2 * \mathrm{M} * \mathrm{r}^{\wedge}\left(-\left(1+\mathrm{b}^{\wedge} 2 *(\mathrm{~d}-3)\right) /\left(1+\mathrm{b}^{\wedge} 2\right)\right)\right.\right.$

$>-2 *$ Lambda/ $\left.\left.\left(1+b^{\wedge} 2 *(d-3)\right)\right), r\right)$;

$$
\begin{aligned}
& U:=r \rightarrow\left(1+\frac{1}{4} \beta^{2}(d-2)\right)^{2} r^{\left(2 \frac{1}{1+1 / 4 \beta^{2}} \overline{(d-2)}\right)} \\
& \left(-2 M r^{\left(-\frac{1+1 / 4 \beta^{2}(d-2)(d-3)}{1+1 / 4 \beta^{2}(d-2)}\right)}-2 \frac{V 0 \mathrm{e}^{(\beta \sqrt{d-2} \phi 0)}}{(d-2)\left(1+\frac{1}{4} \beta^{2}(d-2)(d-3)\right)}\right)
\end{aligned}
$$

Escolhemos valores para os parâmetros

$>\mathrm{k}:=1 ; \mathrm{d}:=6 ;$ beta $:=\operatorname{sqrt}(5) / 2 ; \mathrm{V0}:=-1 ; \mathrm{V} 1:=1 ; \mathrm{M}:=-1 / 10 ; \mathrm{phi} .0:=1 ;$

$d r / d \tau$ é dada por

$>\operatorname{Dr}:=(\operatorname{expand}(\operatorname{simplify}(\operatorname{sqrt}(-2 * F(r)) / \operatorname{diff}(R(r), r))))$;

$$
D r:=\frac{9}{760} \sqrt{19} \sqrt{-200 \mathrm{e}^{(\sqrt{5})}-380 \frac{1}{r^{19 / 9}}+19 r^{2 / 9}\left(\mathrm{e}^{(\sqrt{5})}\right)^{4 / 5}} r^{4 / 9}
$$

Definimos a equação de movimento para a parede de domínio em termos do tempo do bulk $t$

$$
\begin{aligned}
& >\text { Eq1: }=\operatorname{diff}(r(t), t)=(\operatorname{subs}(r=r(t), \operatorname{simplify}(\operatorname{Dr} * \operatorname{simplify}(U(r)) / \operatorname{sqrt}( \\
& \left.\left.\left.\left.>\operatorname{simplify}(U(r))+\operatorname{Dr} r^{\sim} 2\right)\right)\right)\right) ;
\end{aligned}
$$

$$
\begin{aligned}
E q 1 & :=\frac{\partial}{\partial t} \mathrm{r}(t)=\frac{81}{28880} \sqrt{19} \\
& \sqrt{-\frac{200 \mathrm{e}^{(\sqrt{5})} \mathrm{r}(t)^{19 / 9}+380-19 \mathrm{e}^{(4 / 5 \sqrt{5})} \mathrm{r}(t)^{7 / 3}}{\mathrm{r}(t)^{19 / 9}}}
\end{aligned}
$$




$$
\left(19+10 \mathrm{e}^{(\sqrt{5})} \mathrm{r}(t)^{19 / 9}\right) \mathrm{e}^{(-2 / 5 \sqrt{5})} /\left(\mathrm{r}(t)^{7 / 9} \sqrt{\mathrm{r}(t)^{10 / 9}}\right)
$$

E resolvemos-a

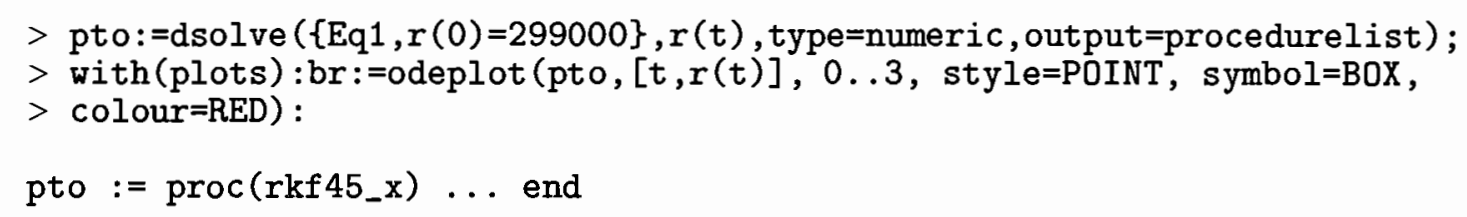

Para as geodésicas temos a equação que segue

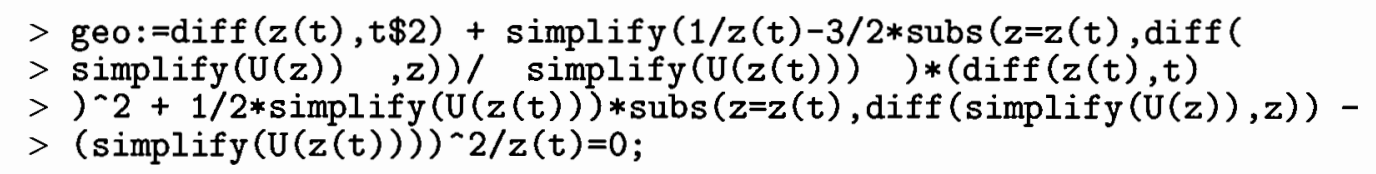

$$
\begin{aligned}
\text { geo } & :=\left(\frac{\partial^{2}}{\partial t^{2}} \mathrm{z}(t)\right)-\frac{1}{6} \frac{(-323+20 \% 1)\left(\frac{\partial}{\partial t} \mathrm{z}(t)\right)^{2}}{\mathrm{z}(t)(19+10 \% 1)} \\
& +\frac{81}{3040} \frac{(19+10 \% 1)\left(-\frac{99}{1520} \frac{19+10 \% 1}{\mathrm{z}(t)^{20 / 9}}+\frac{9}{8} \frac{\mathrm{e}^{(\sqrt{5})}}{\mathrm{z}(t)^{1 / 9}}\right)}{\mathrm{z}(t)^{11 / 9}} \\
& -\frac{6561}{2310400} \frac{(19+10 \% 1)^{2}}{\mathrm{z}(t)^{31 / 9}}=0 \\
& \% 1:=\mathrm{e}^{(\sqrt{5})} \mathrm{z}(t)^{19 / 9}
\end{aligned}
$$

Resolvemos numericamente para certas condições iniciais

$$
\begin{aligned}
& >\text { pgeo1: }=\text { dsolve }(\text { geo }, z(0)=299000, D(z)(0)=-50000\}, z(t) \text {, type=numeric, } \\
& >\text { output=procedurelist) ; geo1:=odeplot (pgeo1, }[t, z(t)], 0.0 .7 \text {, style= } \\
& >\text { POINT, symbol=BOX, colour=BLUE) : } \\
& \text { pgeo1 := proc }\left(\operatorname{rkf45} \_x\right) \ldots \text { end } \\
& >\text { pgeo2: }=\text { dsolve }(\text { geo, } z(0)=299000, D(z)(0)=-100000\}, z(t) \text {, type =numeric, } \\
& >\text { output=procedurelist);geo2:=odeplot (pgeo2, }[t, z(t)], 0 \ldots 1.3 \text {, style }= \\
& >\text { POINT, symbol=BOX, colour=GREEN): } \\
& \text { pgeo2 := proc }\left(\operatorname{rkf} 45_{-} \mathrm{x}\right) \ldots \text { end } \\
& >\text { pgeo3: =dsolve }(\{\text { geo }, z(0)=299000, D(z)(0)=-150000\}, z(t), \text { type }=\text { numeric, } \\
& >\text { output=procedurelist); geo3:=odeplot (pgeo3, }[t, z(t)], 0.1 .8 \text {, style }= \\
& >\text { POINT, symbol=BOX, colour }=\text { YELLOW): } \\
& \text { pgeo3 := proc }\left(\operatorname{rkf45} \_x\right) \ldots \text { end } \\
& >\text { pgeo4: }=\text { dsolve }(\{\text { geo }, z(0)=299000, D(z)(0)=-200000\}, z(t), \text { type=numeric, } \\
& >\text { output=procedurelist); geo4:=odeplot (pgeo4, }[t, z(t)], 0.2 .5 \text {, style }= \\
& >\text { POINT, symbol=BOX, colour=MAGENTA): }
\end{aligned}
$$


pgeo4 := proc $\left(r k f 45_{-} x\right) \ldots$ end

E finalmente apresentamos todas as curvas juntas

$>$ display ( $\{$ geo1, geo2, geo3, geo4,br $\}$, title $={ }^{\prime} \mathrm{M}=-1 / 10, \mathrm{~V} 0=-1, \mathrm{~V} 1=1, \mathrm{ph} i=1$,

$>$ beta $=1 /$ sqrt $(6), z(0)=0.01, z p(0)=.001, .01, .025, .04^{\prime}$, titlefont= [TIMES,

$>$ BOLD , 12], labelf ont $=[$ TIMES, BDLD , 28] , axesfont= [TIMES , BDLD , 18] ) ;

O atraso no tempo é achado da seguinte maneira. Definimos certos parâmetros "del" e "nn", correspondendo ao largo e ao número de retângulos considerados para a integração numérica.

$>\operatorname{del}:=.01 ; \mathrm{nn}:=243$;

Definimos a matriz de integração para a geodésica

$>$ Integrando:=array $(1 \ldots n n):$ for $i$ from 0 by 1 to $(n n-1)$ do

$>$ Integrando $[i+1]:=$ pgeo4 $(i * d e l)$ od: print(Integrando):

e para a parede de domínio

$>$ pto: $=$ dsolve $(\{\mathrm{Eq} 1, \mathrm{r}(0)=299000\}, \mathrm{r}(\mathrm{t})$, type=numeric , output=procedurelist $)$;

$>$ brane:=array $(1 \ldots \mathrm{nn})$ :for 11 from 0 by 1 to $(\mathrm{nn}-1)$ do brane $[11+1]:=$

$>$ pto(ll*del) od: print(brane):

Comparamos os elementos de matriz brane[nn] e Integrandn[nn] para saber se as curvas já cruzaram, em cujo caso existe um atalho.

$>$ brane $[\mathrm{nn}]$; Integrando[nn];

$$
\begin{gathered}
{[t=2.42, \mathrm{r}(t)=511961.5365090940]} \\
{\left[t=2.42, \mathrm{z}(t)=514225.8213991952, \frac{\partial}{\partial t} \mathrm{z}(t)=466362.5073289764\right]}
\end{gathered}
$$

Também precisamos $d \tau / d t$

$>$ dtau_dt:=unapply $\left(\operatorname{factor}\left(U(r) / \operatorname{sqrt}\left(U(r)+(D r)^{\wedge} 2\right)\right), r\right)$;

$d t a u_{-} d t:=$

$$
r \rightarrow \frac{9}{38} \frac{\left(19+10 \mathrm{e}^{(1 / 2 \sqrt{5} \sqrt{4})} r^{19 / 9}\right) \sqrt{19}}{r^{11 / 9} \sqrt{r^{8 / 9}\left(200 \mathrm{e}^{(1 / 2 \sqrt{5} \sqrt{4})}-200 \mathrm{e}^{(\sqrt{5})}+19 r^{2 / 9}\left(\mathrm{e}^{(\sqrt{5})}\right)^{4 / 5}\right)}}
$$

Obtemos o integrando ao longo da trajetória do fóton na parede de domínio

$>$ phot:=array (1..nn): for kk from 1 to $n$ n do phot [kk]:=evalf (subs (

$>$ Integrando [kk] [2], Integrando[kk] [3]\}, subs $(\{r=z(t), d r-d t=$

$\left.\left.\left.>\operatorname{diff}(z(t), t)\},\left(\operatorname{sqrt}\left(U(r)-\left(d r_{-} d t\right)-2 / U(r)\right)\right) / r\right)\right)\right)$ od: print (phot):

e ao longo da trajetória do gráviton no bulk

$>$ grav:=array(1..nn): for qq from 1 to $n$ do grav[qq]:=evalf (subs(

$>$ IIntegrando [qq] [2], brane [qq] [2]\}, $\operatorname{subs}(\{r=z(t), r b=r(t)\}$,

$>-\operatorname{dtau} \operatorname{dt}(\mathrm{r}) /(\mathrm{rb}))$ ) od: $\operatorname{print}($ grav $)$ :

Executamos as integrais

$>$ Rphot: $\left.=\operatorname{del} *\left(1 / 2 * \operatorname{phot}[1]+\operatorname{sum}\left({ }^{\prime} \operatorname{phot}[k]\right]^{\prime},{ }^{\prime}{ }^{\prime}=2 \ldots n n-1\right)+1 / 2 * \operatorname{phot}[n n]\right)$; 


$$
\text { Rphot }:=.004634499363
$$

$>\operatorname{Rgrav}:=\operatorname{del} *\left(1 / 2 * \operatorname{grav}[1]+\operatorname{sum}\left({ }^{\prime} \operatorname{grav}[\mathrm{k}]\right.\right.$ ' , ' $k$ '=2 . $\left.\left.n n-1\right)+1 / 2 * \operatorname{grav}[\mathrm{nn}]\right)$;

$$
\text { Rgrav := -.003945438857 }
$$

E o atraso está dado por

$>$ timedelay:=subs (Integrando[nn] [2], $z(t)) *($ Rphot+Rgrav);

$$
\text { timedelay }:=354.3327047
$$

O tempo da brana é achado integrando a relação entre $d t$ e $d \tau$

$>$ int_t1:=unapply $\left(\right.$ factor $\left(\operatorname{sqrt}\left(\left((U(r))^{\wedge} 2-(D r)^{\wedge} 2 *(\operatorname{dtau} \operatorname{dt}(r))^{\wedge} 2\right) / U(r)\right), r\right)$; int_t1 :=

$$
r \rightarrow \frac{9}{38} \sqrt{19} \sqrt{\frac{\left(19+10 \mathrm{e}^{(1 / 2 \sqrt{5} \sqrt{4})} r^{19 / 9}\right)^{2}}{r^{10 / 3}\left(200 \mathrm{e}^{(1 / 2 \sqrt{5} \sqrt{4})}-200 \mathrm{e}^{(\sqrt{5})}+19 r^{2 / 9}\left(\mathrm{e}^{(\sqrt{5})}\right)^{4 / 5}\right)}}
$$

Definimos a matriz para a integral

$>$ int_t2:=array(1..nn): for $\mathrm{mm}$ from 1 by 1 to $\mathrm{nn}$ do int_t2[mm]:=

$>$ evalf(subs (brane [mm] [2], $\operatorname{subs}(r=r(t)$, int_t1(r)))) od: print(int_t2):

e integramos

$>\operatorname{tdw} 2:=\operatorname{del} *(1 / 2 *$ int_t2 [1] +sum('int_t2 [k]', ' $k$ '=2 . .nn-1)+1/2*int_t2 [nn] ); $t d w 2:=1567.580118$ 


\section{Apêndice D}

\section{Atalhos no Modelo de Brana Dinâmica}

Vamos ilustrar a obtenção de atalhos no modelo descrito no capítulo 7 .

$>$ restart;

A métrica é dada por

$>\mathrm{h}:=$ unapply $\left(\mathrm{k}+\mathrm{z}^{\wedge} 2 / \mathrm{I}^{\wedge} 2-\mathrm{M} / \mathrm{z}^{\wedge} 3+\mathrm{Q}^{\wedge} 2 / \mathrm{z}^{\wedge} \mathrm{G}, \mathrm{z}, \mathrm{k}, \mathrm{M}, \mathrm{Q}\right)$;

$$
h:=(z, k, M, Q) \rightarrow k+\frac{z^{2}}{l^{2}}-\frac{M}{z^{3}}+\frac{Q^{2}}{z^{6}}
$$

$>\mathrm{a}:=\operatorname{unapply}(z / 1, z)$;

$$
a:=z \rightarrow \frac{z}{l}
$$

Usando o sistema (7.5), a equação de movimento da brana é

$>$ op $1:=\operatorname{diff}(\mathrm{R}(\mathrm{t}), \mathrm{t} \$ 2)$;

$$
o p 1:=\frac{\partial^{2}}{\partial t^{2}} \mathrm{R}(t)
$$

$>$ op $2:=1 / 2 * \operatorname{subs}(z=R(t), \operatorname{diff}(h(z, k, M, Q), z)) /(h(R(t), k, M, Q))-3 *$ $>(\operatorname{diff}(R(t), t)) \wedge 4$;

$$
\text { op2 }:=\frac{1}{2} \frac{\left(2 \frac{\mathrm{R}(t)}{l^{2}}+3 \frac{M}{\mathrm{R}(t)^{4}}-6 \frac{Q^{2}}{\mathrm{R}(t)^{7}}\right)\left(\frac{\partial}{\partial t} \mathrm{R}(t)\right)^{4}}{\left(k+\frac{\mathrm{R}(t)^{2}}{l^{2}}-\frac{M}{\mathrm{R}(t)^{3}}+\frac{Q^{2}}{\mathrm{R}(t)^{6}}\right)^{3}}
$$

$>$ op3:=-3*subs $(z=R(t), \operatorname{diff}(h(z, k, M, Q), z)) / h(R(t), k, M, Q) *$

$>(\operatorname{diff}(R(t), t))^{\wedge} 2$; 


$$
\begin{aligned}
& \text { op3 }:=-3 \frac{\left(2 \frac{\mathrm{R}(t)}{l^{2}}+3 \frac{M}{\mathrm{R}(t)^{4}}-6 \frac{Q^{2}}{\mathrm{R}(t)^{7}}\right)\left(\frac{\partial}{\partial t} \mathrm{R}(t)\right)^{2}}{k+\frac{\mathrm{R}(t)^{2}}{l^{2}}-\frac{M}{\mathrm{R}(t)^{3}}+\frac{Q^{2}}{\mathrm{R}(t)^{6}}} \\
& >\text { op4 }:=1 / 2 * h(R(t), k, M, Q) * \operatorname{subs}(z=R(t), \operatorname{diff}(h(z, k, M, Q), z)) \text {; } \\
& \text { op4 }:=\frac{1}{2}\left(k+\frac{\mathrm{R}(t)^{2}}{l^{2}}-\frac{M}{\mathrm{R}(t)^{3}}+\frac{Q^{2}}{\mathrm{R}(t)^{6}}\right)\left(2 \frac{\mathrm{R}(t)}{l^{2}}+3 \frac{M}{\mathrm{R}(t)^{4}}-6 \frac{Q^{2}}{\mathrm{R}(t)^{7}}\right)
\end{aligned}
$$

Da segunda equação em (7.5) podemos obter o valor de $\kappa_{(6)}^{2} \rho$ para substituir na primeira equação do mesmo sistema.

$$
\begin{aligned}
& >\operatorname{rho:}:=8 *(1-(\operatorname{diff}(R(t), t)) \wedge 2 /(h(R(t), k, M, Q)) \sim 2) \sim(-1 / 2) * \\
& >(\operatorname{subs}(z=R(t), \operatorname{diff}(a(z), z)) / a(R(t))+\operatorname{diff}(R(t), t) / \\
& >(h(R(t), k, M, Q)) \sim 2 * \operatorname{diff}(a(R(t)), t) / a(R(t))) ;
\end{aligned}
$$

$$
\rho:=8 \frac{\frac{1}{\mathrm{R}(t)}+\frac{\left(\frac{\partial}{\partial t} \mathrm{R}(t)\right)^{2}}{\left(k+\frac{\mathrm{R}(t)^{2}}{l^{2}}-\frac{M}{\mathrm{R}(t)^{3}}+\frac{Q^{2}}{\mathrm{R}(t)^{6}}\right)^{2} \mathrm{R}(t)}}{\sqrt{1-\frac{\left(\frac{\partial}{\partial t} \mathrm{R}(t)\right)^{2}}{\left(k+\frac{\mathrm{R}^{\prime}(t)^{2}}{i^{2}}-\frac{M}{\mathrm{R}(t)^{3}}+\frac{Q^{2}}{\mathrm{R}(t)^{6}}\right)^{2}}}}
$$

Assim, o lado direito da primeira equação em (7.5) é escrito como

$>$ op $5:=$ rho* $(3+4 *$ omega $) / 8 *(\mathrm{~h}(\mathrm{R}(\mathrm{t}), \mathrm{k}, \mathrm{M}, \mathrm{Q}))^{\sim} 2 *\left(1-(\operatorname{diff}(\mathrm{R}(\mathrm{t}), \mathrm{t}))^{-}-2 /\right.$

$\left.>(\mathrm{h}(\mathrm{R}(\mathrm{t}), \mathrm{k}, \mathrm{M}, \mathrm{Q}))^{\wedge} 2\right)^{\wedge}(3 / 2)$;

$$
\begin{aligned}
o p 5:= & \left(1-\frac{\left(\frac{\partial}{\partial t} \mathrm{R}(t)\right)^{2}}{\left(k+\frac{\mathrm{R}(t)^{2}}{l^{2}}-\frac{M}{\mathrm{R}(t)^{3}}+\frac{Q^{2}}{\mathrm{R}(t)^{6}}\right)^{2}}\right) \\
& \left(\frac{1}{\mathrm{R}(t)}+\frac{\left(\frac{\partial}{\partial t} \mathrm{R}(t)\right)^{2}}{\left(k+\frac{\mathrm{R}(t)^{2}}{l^{2}}-\frac{M}{\mathrm{R}(t)^{3}}+\frac{Q^{2}}{\mathrm{R}(t)^{6}}\right)^{2} \mathrm{R}(t)}\right)(3+4 \omega) \\
& \left(k+\frac{\mathrm{R}(t)^{2}}{l^{2}}-\frac{M}{\mathrm{R}(t)^{3}}+\frac{Q^{2}}{\mathrm{R}(t)^{6}}\right)^{2}
\end{aligned}
$$

Finalmente a equação completa é

$>$ Eq1:=(op1+op2+op3+op4+op5);

$$
E q 1:=\left(\frac{\partial^{2}}{\partial t^{2}} \mathrm{R}(t)\right)+\frac{1}{2} \frac{\left(2 \frac{\mathrm{R}(t)}{l^{2}}+3 \frac{M}{\mathrm{R}(t)^{4}}-6 \frac{Q^{2}}{\mathrm{R}(t)^{7}}\right)\left(\frac{\partial}{\partial t} \mathrm{R}(t)\right)^{4}}{\% 1^{3}}
$$




$$
\begin{aligned}
& -3 \frac{\left(2 \frac{\mathrm{R}(t)}{l^{2}}+3 \frac{M}{\mathrm{R}(t)^{4}}-6 \frac{Q^{2}}{\mathrm{R}(t)^{7}}\right)\left(\frac{\partial}{\partial t} \mathrm{R}(t)\right)^{2}}{\% 1} \\
& +\frac{1}{2} \% 1\left(2 \frac{\mathrm{R}(t)}{l^{2}}+3 \frac{M}{\mathrm{R}(t)^{4}}-6 \frac{Q^{2}}{\mathrm{R}(t)^{7}}\right) \\
& +\left(1-\frac{\left(\frac{\partial}{\partial t} \mathrm{R}(t)\right)^{2}}{\% 1^{2}}\right)\left(\frac{1}{\mathrm{R}(t)}+\frac{\left(\frac{\partial}{\partial t} \mathrm{R}(t)\right)^{2}}{\% 1^{2} \mathrm{R}(t)}\right)(3+4 \omega) \% 1^{2} \\
& \% 1:=k+\frac{\mathrm{R}(t)^{2}}{l^{2}}-\frac{M}{\mathrm{R}(t)^{3}}+\frac{Q^{2}}{\mathrm{R}(t)^{6}}
\end{aligned}
$$

A equação geodésica em termos de $h$ é dada por

$$
\begin{aligned}
& >\text { geo:=diff }(z(t), t \$ 2)+\operatorname{simplify}(1 / z(t)-3 / 2 * \operatorname{subs}(z=z(t) \text {, } \\
& >\operatorname{diff}(h(z, k, M, Q), z)) / h(z(t), k, M, Q)) *(\operatorname{diff}(z(t), t))^{-} 2+1 / 2 * \\
& >h(z(t), k, M, Q) * \operatorname{subs}(z=z(t), \operatorname{diff}(h(z, k, M, Q), z))- \\
& >(h(z(t), k, M, Q))^{-} 2 / z(t)=0 \text {; } \\
& g e o:=\left(\frac{\partial^{2}}{\partial t^{2}} \mathrm{z}(t)\right) \\
& +\frac{1}{2} \frac{\left(2 k l^{2} \mathrm{z}(t)^{6}-4 \mathrm{z}(t)^{8}-11 M l^{2} \mathrm{z}(t)^{3}+20 Q^{2} l^{2}\right)\left(\frac{\partial}{\partial t} \mathrm{z}(t)\right)^{2}}{\mathrm{z}(t)\left(k l^{2} \mathrm{z}(t)^{6}+\mathrm{z}(t)^{8}-M l^{2} \mathrm{z}(t)^{3}+Q^{2} l^{2}\right)} \\
& +\frac{1}{2}\left(k+\frac{\mathrm{z}(t)^{2}}{l^{2}}-\frac{M}{\mathrm{z}(t)^{3}}+\frac{Q^{2}}{\mathrm{z}(t)^{6}}\right)\left(2 \frac{\mathrm{z}(t)}{l^{2}}+3 \frac{M}{\mathrm{z}(t)^{4}}-6 \frac{Q^{2}}{\mathrm{z}(t)^{7}}\right) \\
& -\frac{\left(k+\frac{\mathrm{z}(t)^{2}}{l^{2}}-\frac{M}{\mathrm{z}(t)^{3}}+\frac{Q^{2}}{\mathrm{z}(t)^{6}}\right)^{2}}{\mathrm{z}(t)}=0
\end{aligned}
$$

Agora resolvemos a equação geodésica para um caso dado. Fornecemos primeiramente os parâmetros do modelo,

$$
\text { >mega:=-1; } \mathrm{M}:=1 / 10 ; \mathrm{Q}:=1 / 10 ; \mathrm{k}:=1 ; 1:=\mathrm{I} \text {; }
$$

E construímos as curvas para várias velocidades iniciais.

$$
\begin{aligned}
& >\text { pgeo: }=\text { dsolve }(\{\text { geo, } z(0)=.9, D(z)(0)=-0.03\}, z(t) \text {, type=numeric } \text {, } \\
& >\text { output=procedurelist);geo1:=odeplot (pgeo, }[t, z(t)], 0.5 \text {, style }= \\
& >\text { POINT, symbol=BOX, colour }=\text { BLUE) : } \\
& \text { pgeo := proc }\left(r k f 45 \_x\right) \ldots \text { end } \\
& >\text { pgeo: }=\text { dsolve }(\{\text { geo }, z(0)=.9, D(z)(0)=0\}, z(t), \text { type=numeric, output }= \\
& >\text { procedurelist);geo2:=odeplot (pgeo, }[t, z(t)], 0 . .5 \text {, style=POINT, } \\
& >\text { symbol=BOX, colour=GREEN): } \\
& \text { pgeo := proc }\left(r k f 45 \_x\right) \ldots \text { end } \\
& >\text { pgeo: }=\text { dsolve }(\{g e o, z(0)=.9, D(z)(0)=-.05\}, z(t), \text { type=numeric, output }= \\
& >\text { procedurelist);geo3:=odeplot (pgeo, }[t, z(t)], 0.5, \text { style=POINT, }
\end{aligned}
$$


> symbol=BOX, colour=MAGENTA):

pgeo := proc $\left(r k f 45 \_x\right) \ldots$ end

Resolvemos agora a equação de movimento da brana para os casos $\omega=-1,0,1 / 3$, correspondendo a uma parede de domínio, uma brana dominada por matéria, e uma outra dominada por radiação, respetivamente.

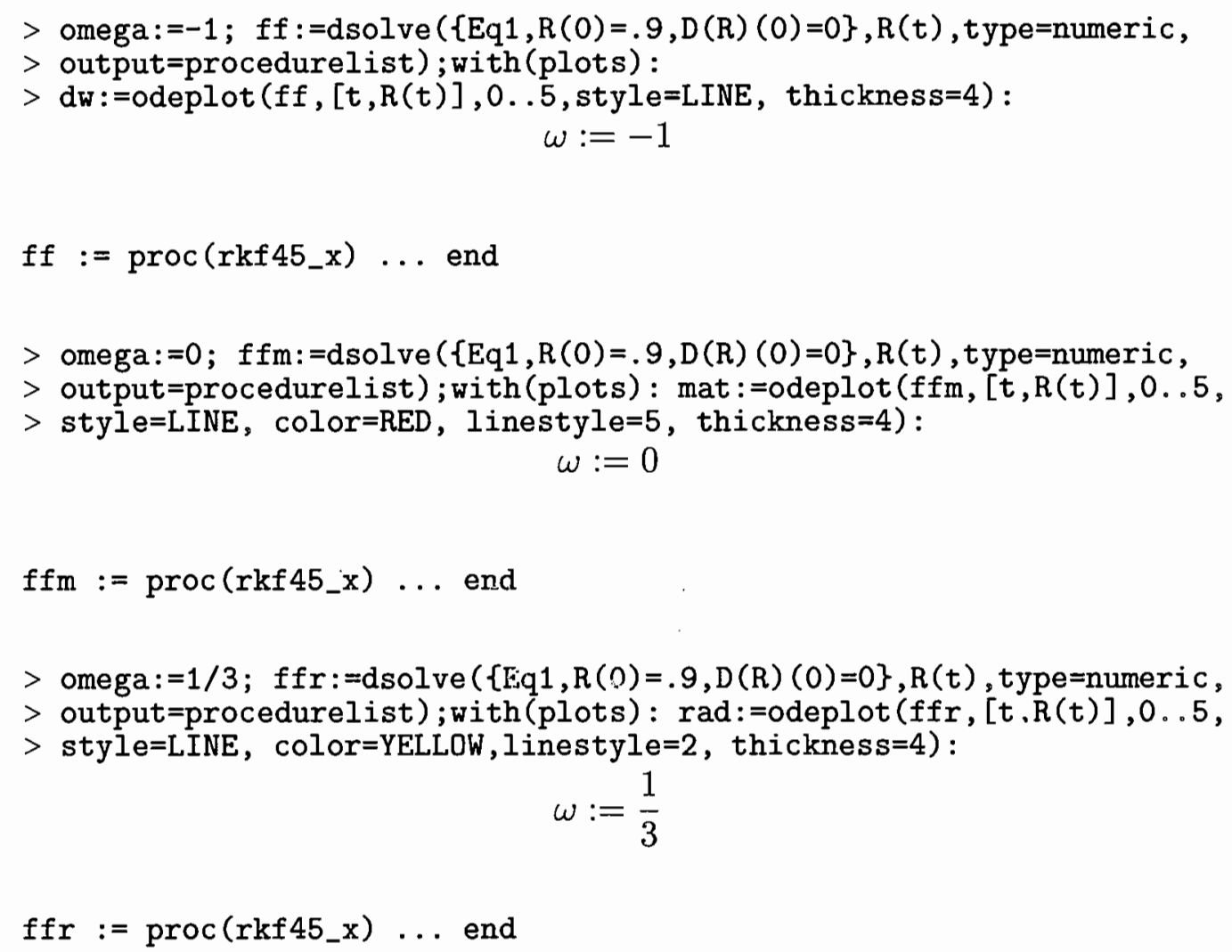

E agora mostramos todas as curvas juntas.

$>$ display ( $\{$ geo1, geo2, geo3, dw , mat, rad $\})$; 


\section{Referências Bibliográficas}

[1] T. Kaluza. Zum unitätsproblem der physik. Sitzungsberichte Preussische Akademie der Wissenschaften, 96:69, 1921.

[2] O. Klein. Quantentheorie und fünfdimensionale relativitätstheorie. Zeitschrift fur Physik, 37:895, 1926.

[3] O. Klein. The atomicity of electricity as a quantum theory law. Nature, 118:516, 1926.

[4] P. Hor̆ava and E. Witten. Heterotic and type I string dynamics from eleven dimensions. Nucl. Phys., B460:506, 1996.

[5] P. Hor̆ava and E. Wittєenı. Eleven-dimensional supergravity on a manifold with boundary. Nucl. Phys., 'B475:94, 1996.

[6] N. Arkani-Hamed, S. Dimopoulos, and G. Dvali. The hierarchy problem and new dimensions at a millimeter. Phys. Lett., B429:263, 1998.

[7] L. Randall and R. Sundrum. A large mass hierarchy from a small extra dimension. Phys. Rev. Lett., 83:3370, 1999.

[8] L. Randall and R. Sundrum. An alternative to compactification. Phys. Rev. Lett., 83:4690, 1999.

[9] E. Witten. The cosmological constant from the viewpoint of string theory. In Marina del Rey 2000: Sources and detection of dark matter and dark energy in the universe, page $27,2000$.

[10] P. Binétruy, C. Deffayet, and D. Langlois. Non-conventional cosmology from a brane-universe. Nucl. Phys., B565:269, 2000.

[11] J. M. Cline, C. Grojean, and G. Servant. Cosmological expansion in the presence of an extra dimension. Phys. Rev. Lett., 83:4245, 1999.

[12] C. Csáki, M. Graesser, C. Kolda, and J. Terning. Cosmology of one extra dimension with localized gravity. Phys. Lett., B462:34, 1999. 
[13] S.-H. Henry Tye and I. Wasserman. A brane world solution to the cosmological constant problem. Phys. Rev. Lett., 86:1682, 2001.

[14] D. Langlois. Evolution of cosmological perturbations in a brane-universe. Phys. Rev. Lett., 86:2212, 2001.

[15] V. Barger, T. Han, T. Li, J. D. Lykken, and D. Marfatia. Cosmology and hierarchy in stabilized warped brane models. Phys. Lett., B488:97, 2000.

[16] N. Arkani-Hamed, S. Dimopoulos, N. Kaloper, and J. March-Russell. Rapid asymmetric inflation and early cosmology in theories with sub-millimeter dimensions. Nucl. Phys., B567:189, 2000.

[17] N. Kaloper and A. Linde. Cosmology vs. holography. Phys. Rev., D60:103509, 1999.

[18] E. Abdalla and L. Alejandro Correa-Borbonet. Holography in an early universe with asymmetric inflation. Phys. Lett., B489:383, 2000.

[19] S. Nojiri and S. D. Odintsov. Brane-world cosmology in higher derivative gravity or warped compactification in the next-to-leading order of ads/cft correspondence. J. High Energy Phys., 07:049, 2000.

[20] S. Nojiri, O. Obregon, and S. D. Odintsov. Cosmological constant and brane new world. Rev. Mex. Fis., 48:19, 2002.

[21] Bin Wang, Elcio Abdalla, and Ru-Keng Su. Friedmann equation and cardy formula correspondence in brane universes. Mod. Phys. Lett., A17:23, 2002.

[22] Bin Wang, Elcio Abdalla, and Ru-Keng Su. Relating friedmann equation to cardy formula in universes with cosmological constant. Phys. Lett., B503:394, 2001.

[23] C. Csáki, M. Graesser, L. Randall, and J. Terning. Cosmology of brane models with radion stabilization. Phys. Rev., D62:045015, 2000.

[24] C. Csáki, J. Erlich, and C. Grojean. Gravitational lorentz violations and adjustment of the cosmological constant in asymmetrically warped spacetimes. Nucl. Phys., B604:312, 2001.

[25] A. Davis, C. Rhodes, and I. Vernon. Branes on the horizon. J. High Energy Phys., 111:015, 2001.

[26] G. Dvali, G. Gabadadze, and M. Porrati. 4d gravity on a brane in 5d minkowski space. Phys. Lett., B485:208, 2000.

[27] C. Deffayet. Cosmology on a brane in minkowski bulk. Phys. Lett., B502:199, 2001. 
[28] C. Deffayet, G. Dvali, and G. Gabadadze. Accelerated universe from gravity leaking to extra dimensions. Phys. Rev., D65:044023, 2002.

[29] H. Ishihara. Causality of the brane universe. Phys. Rev. Lett., 86:381, 2001.

[30] R. Caldwell and D. Langlois. Shortcuts in the fifth dimension. Phys. Lett., B511:129, 2001.

[31] D. J. Chung and K. Freese. Can geodesics in extra dimensions solve the cosmological horizon problem? Phys. Rev., D62:063513, 2000.

[32] D. J. Chung, E. W. Kolb, and A. Riotto. Extra dimensions present a new flatness problem. Phys. Rev., D65:083516, 2002.

[33] E. Abdalla, B. Cuadros-Melgar, S. Feng, and B. Wang. Shortest cut in brane cosmology. Phys. Rev., D65:083512, 2002.

[34] E. Abdalla, A. Casali, and B. Cuadros-Melgar. Shortcuts for graviton propagation in a six-dimensional brane world model. Nucl. Phys., B644:201, 2002.

[35] M. A. Clayton and J. W. Moffat. Dynamical mechanism for varying light velocity as a solution to cosmological problems. Phys. Lett., B460:263, 1999.

[ڤ̂̉] M. A. Clayton and J. W. Moffat. Scalar-tensor gravity theory frr dynamical light velocity. Phys. Lett., B477:269, 2000.

[37] G. Starkman, D. Stojkovic, and M. Trodden. Homogeneity, flatness and "large" extra dimensions. Phys. Rev. Lett., 87:231303, 2001.

[38] G. Starkman, D. Stojkovic, and M. Trodden. Large extra dimensions and cosmological problems. Phys. Rev., D63:103511, 2001.

[39] D. J. Chung and K. Freese. Cosmological challenges in theories with extra dimensions and remarks on the horizon problem. Phys. Rev., D61:023511, 2000.

[40] E. Abdalla and A. G. Casali. Shortcuts in a dynamical universe and the horizon problem. hep-th/0208008.

[41] J. W. Moffat. Variable speed of light cosmology: An alternative to inflation. hep-th/0208122.

[42] E. W. Kolb and M. S. Turner. The Early Universe. Addison-Wesley, New York, 1990.

[43] E. Abdalla and B. Cuadros-Melgar. Shortcuts in domain walls and the horizon problem. Phys. Rev., D67:084012, 2003.

[44] B. Cuadros-Melgar. Non-linear brane dynamics in a six dimensional model. hepth/0303131. 
[45] I. Antoniadis, N. Arkani-Hamed, S. Dimopoulos, and G. Dvali. New dimensions at a millimeter to a fermi and superstrings at a TeV. Phys. Lett., B436:257, 1998.

[46] W. Israel. Singular hypersurfaces and thin shells in general relativity. Nuovo Cimento, B44:1, 1966.

[47] P. Binétruy, C. Deffayet, U. Ellwanger, and D. Langlois. Brane cosmological evolution in a bulk with cosmological constant. Phys. Lett., B477:285, 2000.

[48] S. S. Feng and C. G. Huang. Can dirac observability apply to gravitational systems? Int. J. Theor. Phys., 36:1179, 1997.

[49] A. G. Reiss et. al. Observational evidence from supernovae for an accelerating universe and a cosmological constant. Astrophys. J., 116:1009, 1998.

[50] S. Perlmutter et. al. Measurements of Omega and Lambda from 42 highredshift supernovae. Astrophys. J., 517:565, 1999.

[51] D. Langlois. Brane cosmology: An introduction. Prog.Theor.Phys.Suppl., 148:181, 2003.

[52] S. Mukohyema, T. Shiromizu, and K. I. Maeda. Global structure of exact cosmological solutions in the brane world. Phys. Rev., D62:024028, 2000. Erratum-ibid. 603 (2901) 029901.

[53] A. Kehagias and E. Kiritsis. Mirage cosmology. J. High Energy Phys., 9911:022, 1999.

[54] P. Kanti, R. Madden, and K. A. Olive. A 6-d brane world model. Phys. Rev., D64:044021, 2001.

[55] G. Gibbons and S. Hawking. Cosmological event horizons, thermodynamics, and particle creation. Phys. Rev., D15:2738, 1977.

[56] H. A. Chamblin and H. S. Reall. Dynamic dilatonic domain walls. Nucl.Phys., B562:133, 1999.

[57] P. Binétruy, C. Deffayet, and D. Langlois. The radion in brane cosmology. Nucl. Phys., B615:219, 2001.

[58] C. Charmousis, R. Gregory, and V. A. Rubakov. Wave function of the radion in a brane world. Phys. Rev., D62:067505, 2000.

[59] S. Mukherji and M. Peloso. Bouncing and cyclic universes from brane models. Phys. Lett., B547:297, 2002.

[60] A. J. Mevdev. Bad news on the brane. hep-th/0205251. 
[61] P. Kanti and K. Tamvakis. Challenges and obstacles for a bouncing universe in brane models. Technical report, CERN-TH, 2003.

[62] P.J. Steinhardt and N. Turok. The cyclic universe: An informal introduction. Science, 296:1436, 2002.

[63] P. de Bernardis et. al. A flat universe from high-resolution maps of the cosmic microwave background radiation. Nature, 404:955, 2000.

[64] P. de Bernardis et. al. A measurement of omega from the north american test flight of boomerang. Astrophys. J. Lett., 536:L63, 2000.

[65] R. Stompor et. al. Cosmological implications of the maxima-i high resolution cosmic microwave background anisotropy measurement. Astrophys. J. Lett., 561:L7, 2001.

[66] C. L. Bennett et. al. Wilkinson Microwave Anisotropy Probe. 2003. http://lambda.gsfc.nasa.gov/product/map/map_bibliography.cfm.

[67] G. Giudice, E. Kolb, J. Lesgourgues, and A. Riotto. Transdimensional physics and inflation. Technical report, CERN-TH, 2002. 